\title{
Microfabrication of an Implantable Silicone Microelectrode Array for an Epiretinal Prosthesis
}

M. N. Maghribi

June 10, 2003

Lawrence

Livermore

National

Laboratory 
This document was prepared as an account of work sponsored by an agency of the United States Government. Neither the United States Government nor the University of California nor any of their employees, makes any warranty, express or implied, or assumes any legal liability or responsibility for the accuracy, completeness, or usefulness of any information, apparatus, product, or process disclosed, or represents that its use would not infringe privately owned rights. Reference herein to any specific commercial product, process, or service by trade name, trademark, manufacturer, or otherwise, does not necessarily constitute or imply its endorsement, recommendation, or favoring by the United States Government or the University of California. The views and opinions of authors expressed herein do not necessarily state or reflect those of the United States Government or the University of California, and shall not be used for advertising or product endorsement purposes.

This work was performed under the auspices of the U.S. Department of Energy by University of California, Lawrence Livermore National Laboratory under Contract W-7405-Eng-48. 
MICROFABRICATION OF AN IMPLANTABLE SILICONE MICROELECTRODE ARRAY FOR AN EPIRETINAL PROSTHESIS

By

MARIAM NADER MAGHRIBI

B.S. (Georgia Institute of Technology) 1997

\section{DISSERTATION}

Submitted in partial satisfaction of the requirements for the degree of

DOCTOR OF PHILOSOPHY

in

Biomedical Engineering

in the

OFFICE OF GRADUATE STUDIES

of the

UNIVERSITY OF CALIFORNIA

DAVIS

Approved:

Committee in Charge

2003 


\begin{abstract}
Millions of people suffering from diseases such as retinitis pigmentosa and macular degeneration are legally blind due to the loss of photoreceptor function. Fortunately a large percentage of the neural cells connected to the photoreceptors remain viable, and electrical stimulation of these cells has been shown to result in visual perception. These findings have generated worldwide efforts to develop a retinal prosthesis device, with the hope of restoring vision. Advances in microfabrication, integrated circuits, and wireless technologies provide the means to reach this challenging goal. This dissertation describes the development of innovative silicone-based microfabrication techniques for producing an implantable microelectrode array. The microelectrode array is a component of an epiretinal prosthesis being developed by a multi-laboratory consortium. This array will serve as the interface between an electronic imaging system and the human eye, directly stimulating retinal neurons via thin film conducting traces.
\end{abstract}

Because the array is intended as a long-term implant, vital biological and physical design requirements must be met. A retinal implant poses difficult engineering challenges due to the size of the intraocular cavity and the delicate retina. Not only does it have to be biocompatible in terms of cytotoxicity and degradation, but it also has to be structurally biocompatible, with regard to smooth edges and high conformability; basically mimicking the biological tissue. This is vital to minimize stress and prevent physical damage to the retina. Also, the device must be robust to withstand the forces imposed on it during fabrication and implantation. In order to meet these biocompatibility needs, the use of non-conventional microfabrication materials such as 
silicone is required. This mandates the enhancement of currently available polymerbased fabrication techniques and the development of new microfabrication methods.

Through an iterative process, devices were designed, fabricated, tested and implanted into a canine eye. Metal traces were embedded within a thin substrate fabricated using poly (dimethyl siloxane) (PDMS), an inert biocompatible elastomeric material with high oxygen permeability and low water permeability. Due to its highly conformable nature, PDMS contacted the curved retinal surface uniformly. Fundamental material characteristics were examined to develop reliable and repeatable fabrication processes. 


\section{ACKNOWLEDGMENTS}

My work on the retinal prosthesis project was conducted at the Lawrence Livermore National Laboratory Center for Microtechnology. The retinal prosthesis project is a collaborative effort between five national laboratories, three universities, and a private company supported by the Department Of Energy Medical Sciences Division, Office of Biological and Environmental Research and was conducted under the auspices of the U.S. Department of Energy by Lawrence Livermore National Laboratory, contract number W-7405-ENG-48. I would like to express my deep gratitude and appreciation to our collaborators and the leaders of the DOE retinal prosthesis project team, Dr. Mark Humayun and Dr. Eli Greenbaum, for bringing together this consortium and allowing me to participate in such a phenomenal effort.

April 1998, my graduate career began with a phone call from Dr. Abdul Barakat at $11: 00 \mathrm{pm}$ that would lead me to embark on a psychologically adventurous and academically gratifying journey. In retrospect it was one of the best phone calls I have ever received and in essence the gateway to unexpected and exciting new opportunities and adventures. For that thank you Dr. Barakat. I would like to thank my research advisor Dr. Dennis Matthews for courageously allowing me to be one of his students and for his guidance, support, encouragement, and for introducing me to my mentor Dr. Peter Krulevitch at LLNL. I would like to thank Peter for providing me with the golden opportunity to work with extraordinary people on cutting edge scientific research. Working with Peter has been the most enriching and fulfilling experience in my graduate career, actually in my life. I would like to thank Peter for believing in me and for constantly challenging me. I would also like to thank him for his continuous encouragement and support. I highly admire and respect his optimism, enthusiasm, charisma, and great leadership; these qualities radiate confidence within our group and cultivate innovation.

Conducting my research at LLNL offered me numerous resources and allowed me to interact with world-class researchers. I would like to express my gratitude and appreciation to all those who made my graduate experience at LLNL invaluable. Julie Hamilton for mentoring me in microfabrication and sharing her expertise and friendship; her open-mindedness, genuineness and kind-heart made working with her a pleasure and an honor. Tom Wilson for his advice, support and encouragement throughout my research. Cheryl Stockton for her technical support and for always humoring me. My office-mate and trouble-shooting partner, Klint Rose, for helping me understand the "BOSS" through sign language. Courtney Davidson for his relentless support and encouragement. Armando Tovar for keeping an "eye" on the project. Alex Papavasiliou for his tea, thesaurus and conscience mind. Art Nelson and Kuang-Jen Wu for their assistance and expertise with surface analysis work and for answering my never-ending questions. There are many more people at LLNL who have been a positive influence on my graduate experience and hopefully the future will provide me the opportunity to work more closely with them: Amy Wang, Bill Benett, Dennis Polla, Kirk Seward, Mark Strauch. Annette MacIntyre, Mike Pocha, Greg Suski, Rod Keifer, Eddie Scott, and Paul Stratton. I would also like to thank my committee members, Drs. Morse and Shackelford, for their patience and guidance throughout my dissertation process. 
I would like to give a very special thanks to my mom, Afaf, who has been my biggest supporter and who has miraculously endured and survived living with me the past sleepless few months. She is the one who really deserves this Ph.D; her blessings and encouragement made this journey a smooth one. I appreciate and cherish all of the sacrifices and hardships she has endured in her life in order to provide her children with better opportunities to better themselves. Of course I cannot forget to thank all my family members especially my siblings, Zaki, Ziad, and Zeina, who hassled me for the past few months...I mean motivated and encouraged me to finish my dissertation. I also like to thank Susana and Sofia Krulevitch for putting up with my pesky phone calls regarding my dissertation and to Zara Sieh for relentlessly pushing me to set a TIMETABLE. Last but definitely not least I would like to thank all my friends especially Deborah, Nancy, Yassal, Zeina, and Kamal for putting up with me and keeping me sane in the midst of all the insanity.

During my graduate career and particularly in the past three years I have experienced the extremes of all emotions, at many times simultaneously. I must admit the combination of working on a project that I love with great people and finding solace amongst the world's pulse of conscience (antiwar protestors) made these past few years survivable in what seemed to be the darkest abyss of humanity. Academically and professionally I could not have wished for a more enriching experience. However as my mind frolicked in knowledge and innovation, my heart grieved and could not fully savor these rewarding accomplishments in the wake of ongoing austerities and tragedies fueled by mankind's greed, hypocrisy, injustice, arrogance and plain ignorance. Despite my frustration, I am still optimistic that change is possible if we simply acknowledging each other's natural right of existence and by treating one another with respect and dignity. 


\section{ACKNOWLEDGMENTS}

My work on the retinal prosthesis project was conducted at the Lawrence Livermore National Laboratory Center for Microtechnology. The retinal prosthesis project is a collaborative effort between five national laboratories, three universities, and a private company supported by the Department Of Energy Medical Sciences Division, Office of Biological and Environmental Research and was conducted under the auspices of the U.S. Department of Energy by Lawrence Livermore National Laboratory, contract number W-7405-ENG-48. I would like to express my deep gratitude and appreciation to our collaborators and the leaders of the DOE retinal prosthesis project team, Dr. Mark Humayun and Dr. Eli Greenbaum, for bringing together this consortium and allowing me to participate in such a phenomenal effort.

April 1998, my graduate career began with a phone call from Dr. Abdul Barakat at 11:00 pm that would lead me to embark on a psychologically adventurous and academically gratifying journey. In retrospect it was one of the best phone calls I have ever received and in essence the gateway to unexpected and exciting new opportunities and adventures. For that thank you Dr. Barakat. I would like to thank my research advisor Dr. Dennis Matthews for courageously allowing me to be one of his students and for his guidance, support, encouragement, and for introducing me to my mentor Dr. Peter Krulevitch at LLNL. I would like to thank Peter for providing me with the golden opportunity to work with extraordinary people on cutting edge scientific research. Working with Peter has been the most enriching and fulfilling experience in my graduate career, actually in my life. I would like to thank Peter for believing in me and for constantly challenging me. I would also like to thank him for his continuous encouragement and support. I highly admire and respect his optimism, enthusiasm, charisma, and great leadership; these qualities radiate confidence within our group and cultivate innovation.

Conducting my research at LLNL offered me numerous resources and allowed me to interact with world-class researchers. I would like to express my gratitude and appreciation to all those who made my graduate experience at LLNL invaluable. Julie Hamilton for mentoring me in microfabrication and sharing her expertise and friendship; her open-mindedness, genuineness and kind-heart made working with her a pleasure and an honor. Tom Wilson for his advice, support and encouragement throughout my research. Cheryl Stockton for her technical support and for always humoring me. My office-mate and trouble-shooting partner, Klint Rose, for helping me understand the "BOSS" through sign language. Courtney Davidson for his relentless support and encouragement. Armando Tovar for keeping an "eye" on the project. Alex Papavasiliou for his tea, thesaurus and conscience mind. Art Nelson and Kuang-Jen Wu for their assistance and expertise with surface analysis work and for answering my never-ending questions. There are many more people at LLNL who have been a positive influence on my graduate experience and hopefully the future will provide me the opportunity to work more closely with them: Amy Wang, Bill Benett, Dennis Polla, Kirk Seward, Mark Strauch. Annette MacIntyre, Mike Pocha, Greg Suski, Rod Keifer, Eddie Scott, and Paul Stratton. I would also like to thank my committee members, Drs. Morse and Shackelford, for their patience and guidance throughout my dissertation process. 
I would like to give a very special thanks to my mom, Afaf, who has been my biggest supporter and who has miraculously endured and survived living with me the past sleepless few months. She is the one who really deserves this Ph.D; her blessings and encouragement made this journey a smooth one. I appreciate and cherish all of the sacrifices and hardships she has endured in her life in order to provide her children with better opportunities to better themselves. Of course I cannot forget to thank all my family members especially my siblings, Zaki, Ziad, and Zeina, who hassled me for the past few months...I mean motivated and encouraged me to finish my dissertation. I also like to thank Susana and Sofia Krulevitch for putting up with my pesky phone calls regarding my dissertation and to Zara Sieh for relentlessly pushing me to set a TIMETABLE. Last but definitely not least I would like to thank all my friends especially Deborah, Nancy, Yassal, Zeina, and Kamal for putting up with me and keeping me sane in the midst of all the insanity.

During my graduate career and particularly in the past three years I have experienced the extremes of all emotions, at many times simultaneously. I must admit the combination of working on a project that I love with great people and finding solace amongst the world's pulse of conscience (antiwar protestors) made these past few years survivable in what seemed to be the darkest abyss of humanity. Academically and professionally I could not have wished for a more enriching experience. However as my mind frolicked in knowledge and innovation, my heart grieved and could not fully savor these rewarding accomplishments in the wake of ongoing austerities and tragedies fueled by mankind's greed, hypocrisy, injustice, arrogance and plain ignorance. Despite my frustration, I am still optimistic that change is possible if we simply acknowledging each other's natural right of existence and by treating one another with respect and dignity. 


\section{LIST OF TABLES}

CHAPTER 3: SPECIFICATIONS AND FABRICATION TOOLBOX .62

3.1. Implant specifications................................................64

3.2. Comparison of Young's Modulus of various materials............................66

3.3. Sylgard ${ }^{\circledR} 184$ (Dow Corning Midland, MI) Characteristics.........................69

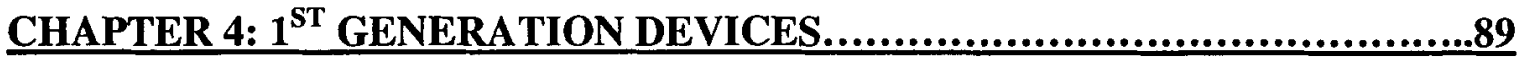

4.1. PDMS thicknesses...................................................96

4.2. Adhesion test and electrical continuity results ............................... 106

CHAPTER 5: $2^{\text {ND }}$ GENERATION DEVICES...............................111

5.1. Adhesion and electrical continuity results................................. 128

CHAPTER 6: SURFACE ANALYSIS AND CURING KINETICS................139

6.1. PDMS peak intensity inside patterned hole using ToF-SIMS analysis.............152

6.2. XPS Compositional Analysis (Atomic \%) of the PDMS Surface.....................156

6.3. Swelling and extraction data using toluene....................................... 161

6.4. Young's Modulus at different cure times and temperatures.........................163 


\section{LIST OF FIGURES}

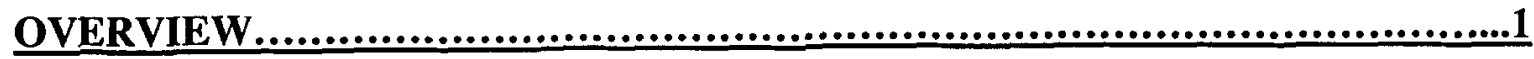

0.1. Diagram of a retinal prosthesis......................................................

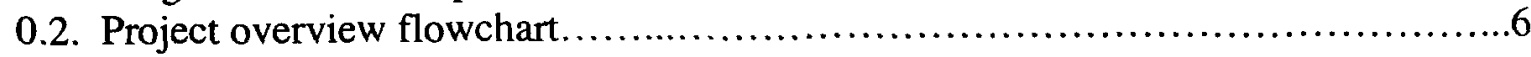

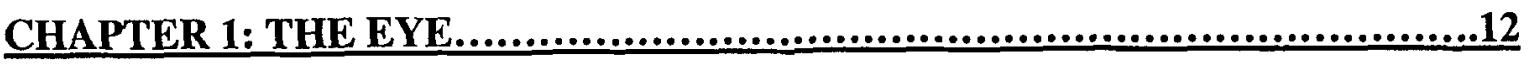

1.1. Anatomy of the eye ...............................................................

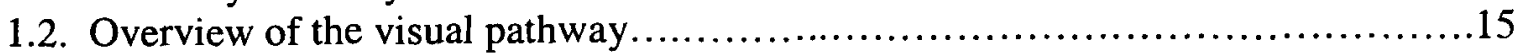

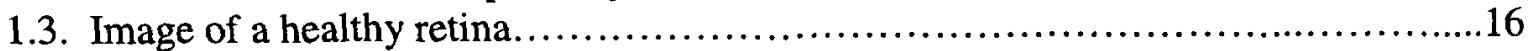

1.4. Illustration of retinal sensory photoreceptors...................................17

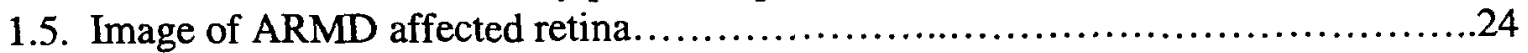

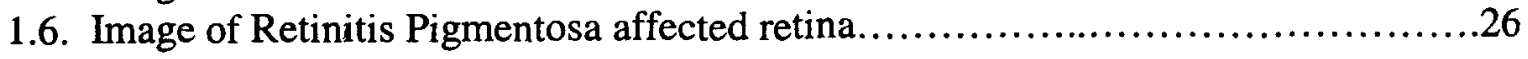

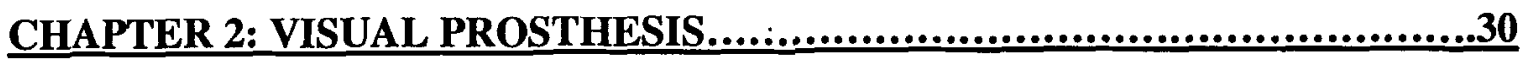

2.1. Proposed system diagram........................................................ 31

2.2. Illustration of epiretinal vs. subretinal prosthesis................................44

2.3. Silicone encapsulated array of 25 electrodes.................................... 47

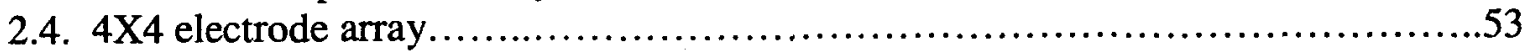

\section{CHAPTER 3: SPECIFICATIONS AND FABRICATION TOOLBOX _............62}

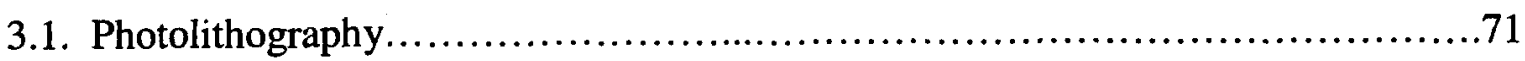

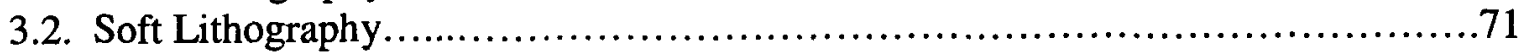

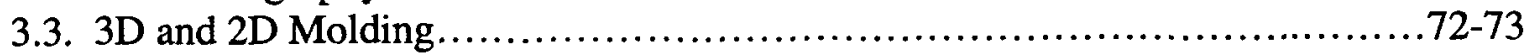

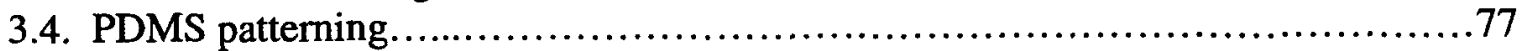

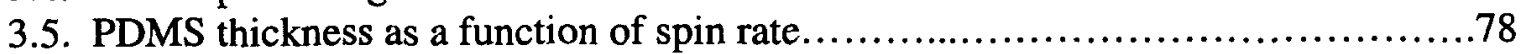

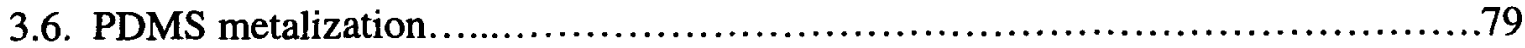

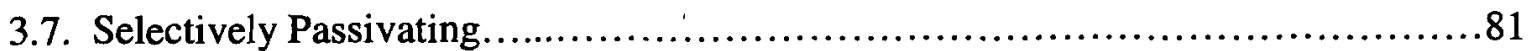

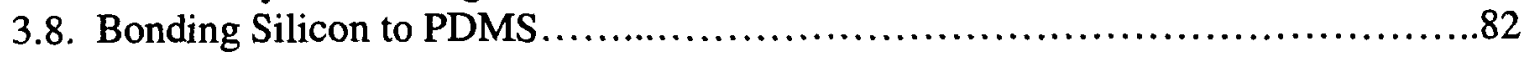

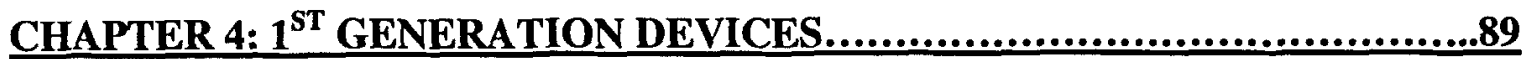

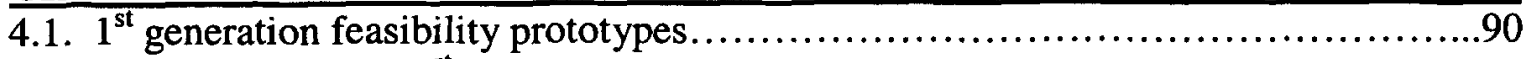

4.2. Illustrations of the $1^{\text {st }}$ generation fabrication design plan ......................92-93

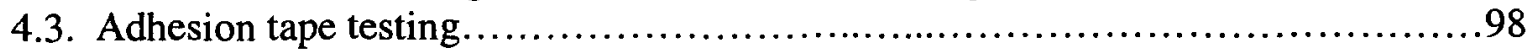

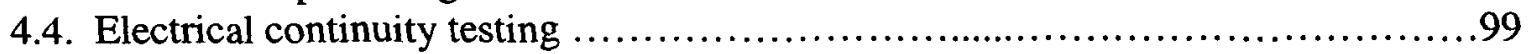

4.5. PDMS soak test prototype.................................................. 100

4.6. Evaluation prototype for surgical implantation.........................102-103

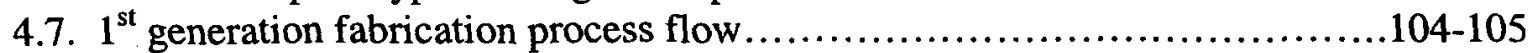

4.8. Acute implant of PDMS test structure in the eye of a dog.......................107

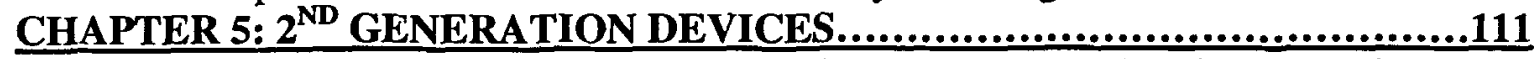

5.1. Metal traces embedded within two PDMS layers are exposed in the electrode and contact pad regions.................................................................... 
5.2. Loop and electrode design

113

5.3. Process flow for $2^{\text {nd }}$ generation devices.......................................

5.4. PC board and glass interconnect design....................................... 119

5.5. Feasibility prototypes........................................................ 120

5.6. Evaluation prototype of $2^{\text {nd }}$ generation surgical implantation device..............123

5.7. The electrode with micro-molded reinforcing ribs.............................125

5.8. SEM of deposited metal traces on

PDMS ........................................1255.9. Electroplating

images..............................................................126

5.10. SEM of selective passivation using membrane transfer approach................126

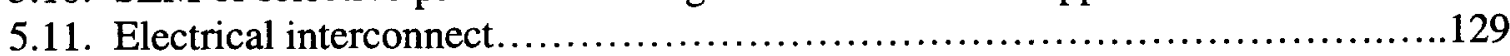

5.12. $2^{\text {nd }}$ generation chronic implant .................................................

5.13. Scanned OCT images...................................................132-134

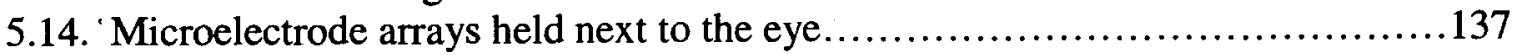

CHAPTER 6: SURFACE ANALYSIS AND CURING KINETICS................139

6.1. Schematic of the Time-of-Flight Secondary Ion Mass Spectrometry...............141

6.2. Schematic of the X-ray photoelectron spectroscopy .............................143

6.3. Rheometric dynamic spectrometer (model RDS-2) for dynamic mechanical analysis

testing........................................................................... 146

6.4. ToF-SIMS spectra of characteristic PDMS.............................150-151

6.5. Illustration of patterned PDMS film on gold-coated silicon wafer.................152

6.6. ToF-SIMS analysis conducted on patterned PDMS hole.........................153

6.7. Positive ion TOF-SIMS spectra obtained from the sample with no $\mathrm{O}_{2}$ plasma

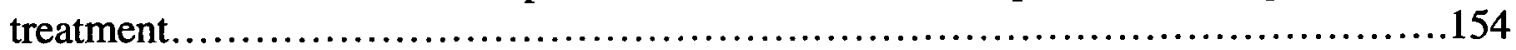

6.8. Positive ion TOF-SIMS spectra for sample with $\mathrm{O}_{2}$ plasma treatment..............155

6.9. XPS survey spectra of PDMS with and without $\mathrm{O}_{2}$ plasma treatment..............157

6.10. XPS C $1 \mathrm{~s}$ core-level spectra for the as-cured and $\mathrm{O}_{2}$ plasma processed PDMS...158

6.11. XPS $\mathrm{O} 1 \mathrm{~s}$ core-level spectra for the as-cured and $\mathrm{O}_{2}$ plasma processed PDMS...159

6.12. XPS Si $2 p$ core-level spectra for the as cured and $\mathrm{O}_{2}$ plasma processed PDMS...159

6.13. XPS survey spectra of PDMS with $\mathrm{O}_{2}$ plasma treatment due to aging ............160

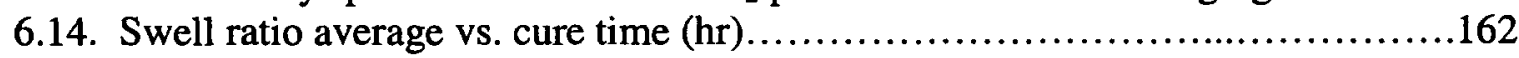

6.15. Percent extractable average vs. cure time (hr.)..............................162

6.16. Dynamic (elastic) storage modulus of PDMS ...............................163

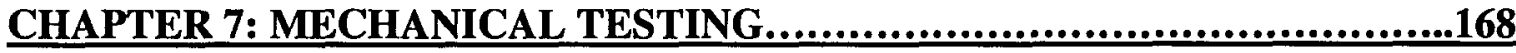

7.1. Gold traces patterned onto PDMS........................................169

7.2. Reference axis on the retina with $\mathrm{z}$ being normal to the surface ..................170

7.3. Straight and serpentine leads tested..........................................172

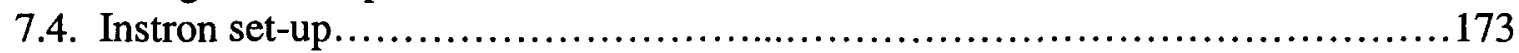

7.5. Illustration of the manual strain measurement set-up...........................174

7.6. Residual stress ratio as a function of thickness ratio...........................176

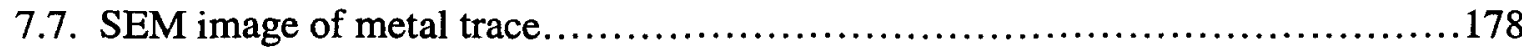

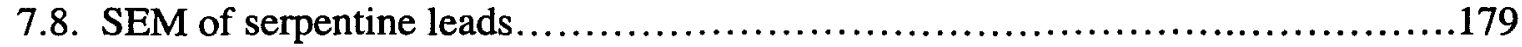


7.9. Evolution of residual stress during curing of PDMS

7.10. Experiment-1 keeping PDMS thickness and gold thickness constant and varying

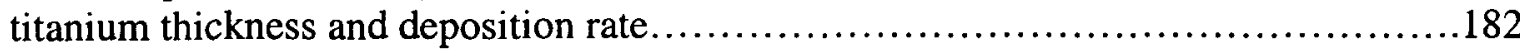

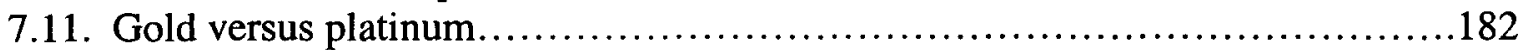

7.12. Schematic of possible future designs to improve stretchablity of the next generation

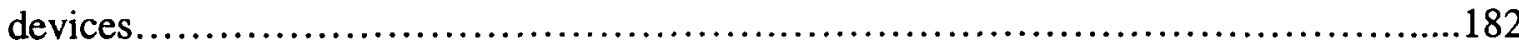

CHAPTER 8: CONCLUSION AND FUTURE DIRECTIONS $\ldots \ldots \ldots \ldots \ldots \ldots \ldots \ldots \ldots \ldots 190$

8.1. OctoPDMS overall concept.................................................. 191 


\section{TABLE OF CONTENTS}

ABSTRACT

DEDICATION. ...ii

ACKNOWLEDGMENTS.

LIST OF FIGURES. ..$x i$

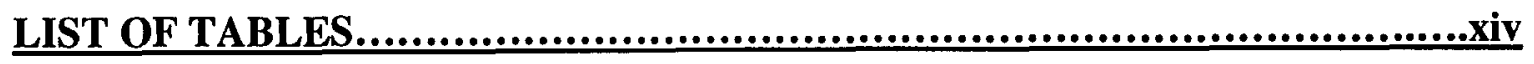

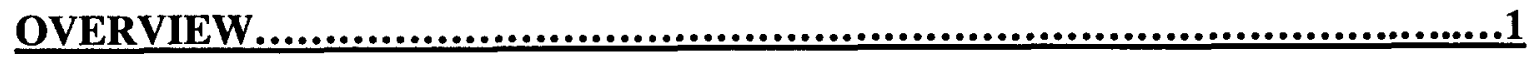

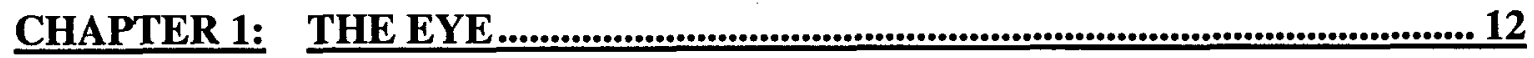

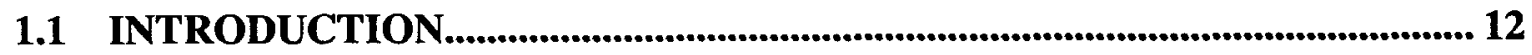

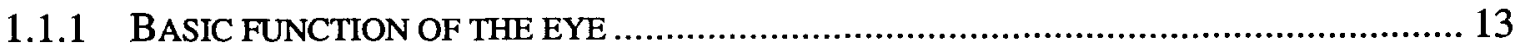

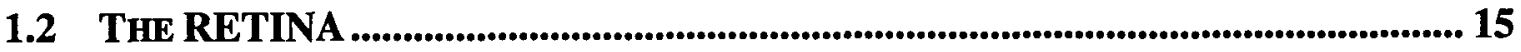

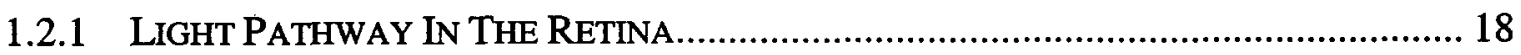

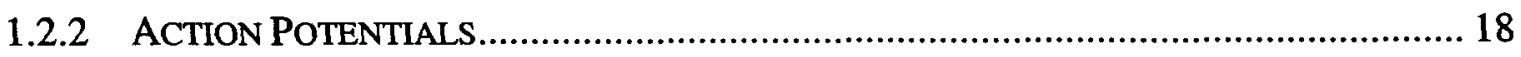

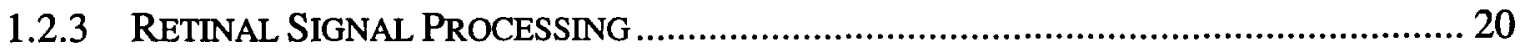

1.3 PHOTORECEPTORS (CONES AND RODS).................................................................... 21

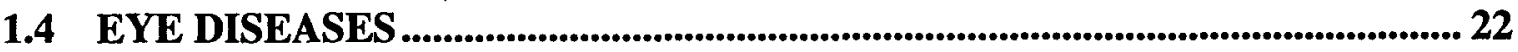

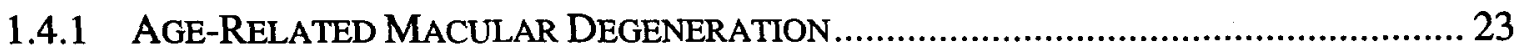

1.4.1.1 Dry Age-Related Macular Degeneration ..................................................... 24

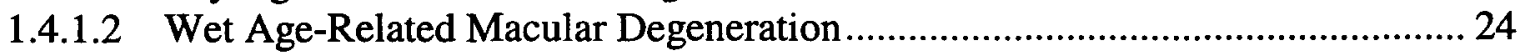

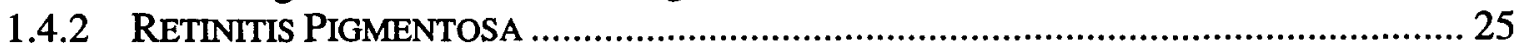

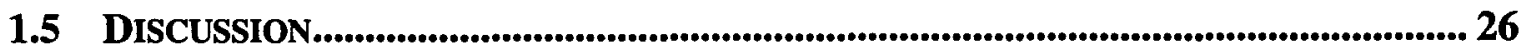

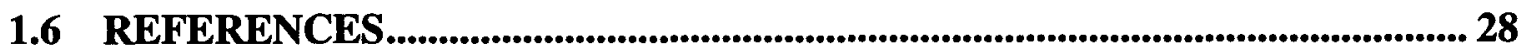

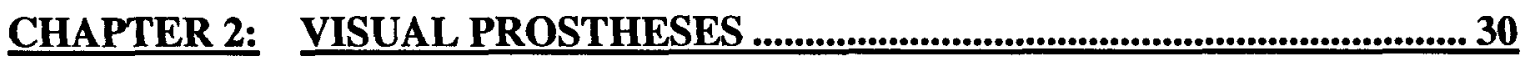

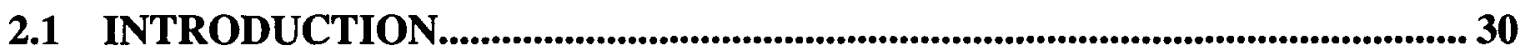

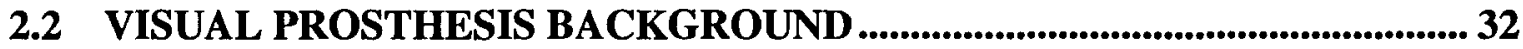

2.3 TYPES OF VISUAL PROSTHESIS ................................................................ 34

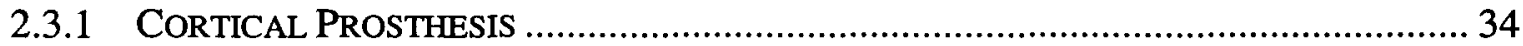

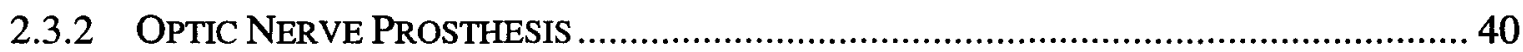

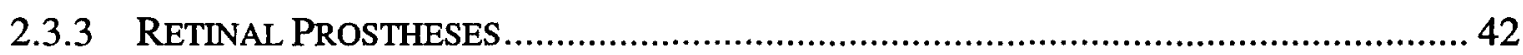

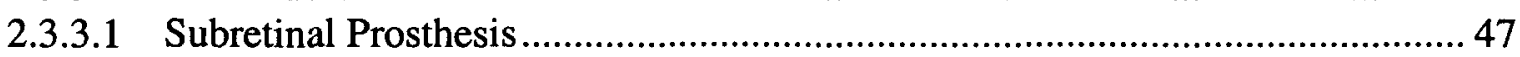

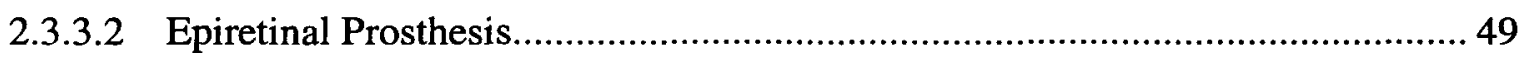

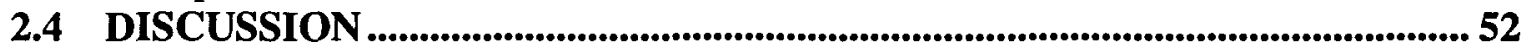

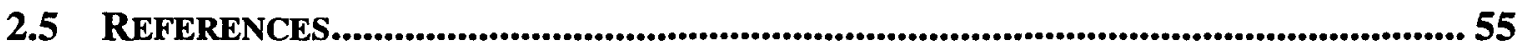


CHAPTER 3: SPECIFICATIONS AND FABRICATION TOOLBOX ...............62

3.1 INTRODUCTION........................................................................................................ 62

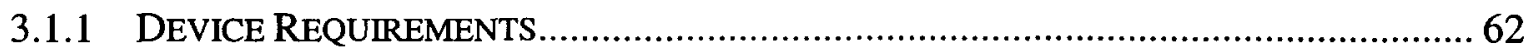

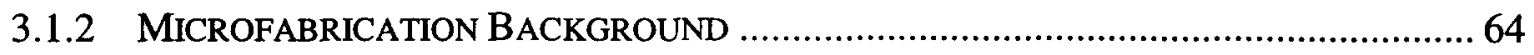

3.2 FABRICATION TOOLBOX .............................................................................. 67

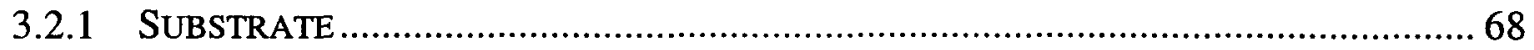

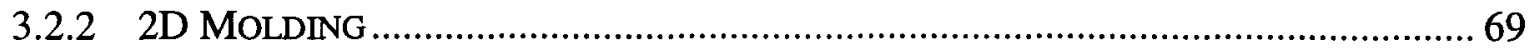

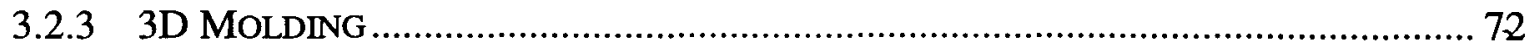

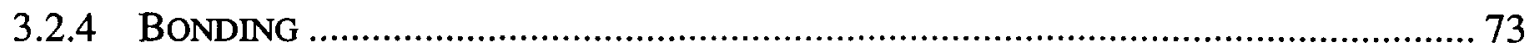

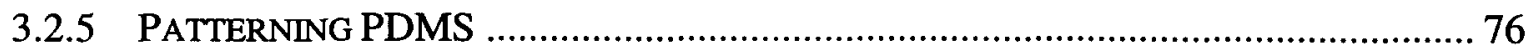

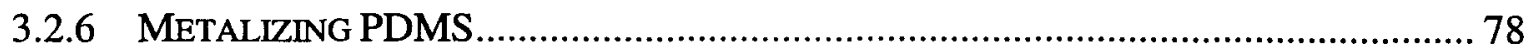

3.2.7 SELECTIVE PASSIVATION LAYER ……………............................................ 79

3.2.8 INTEGRATING EXTERNAL COMPONENTS ......................................................... 81

3.3 DISCUSSION ............................................................................................................................. 82

3.4 REFERENCES.................................................................................................................... 83

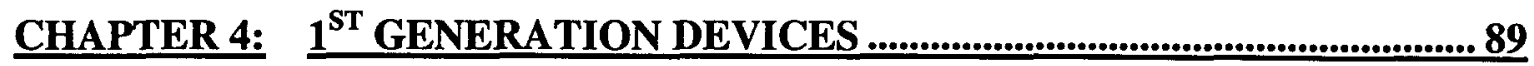

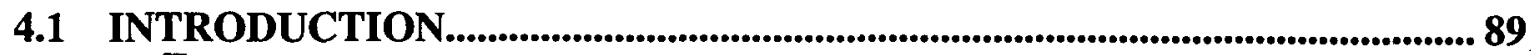

4.1.1 $1^{\text {ST }}$ GENERATION MICROELECTRODE ARRAY FABRICATION PROCESS PLAN ........... 90

4.2 FABRICATION ..............................................................................................................99

4.2.1 PROTOTYPE-1 ELECTRODE DEVELOPMENT.......................................................... 93

4.2.2 PRototyPE-2 SOAK TEST AND PROTOTYPE-3 IMPLANTATION................................. 94

4.3 EXPERIMENTAL APPROACH ....................................................................................995

4.3.1 PDMS THICKNESS CHARACTERIZATION ………............................................... 95

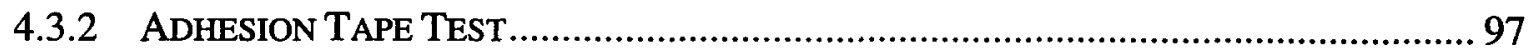

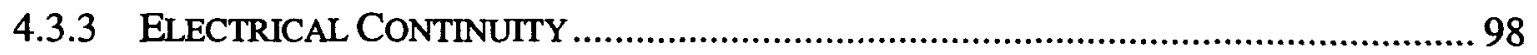

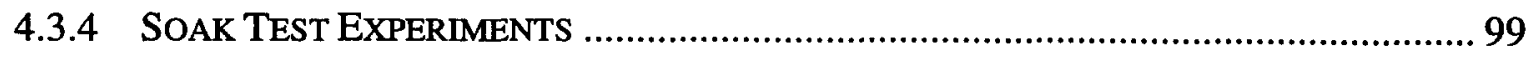

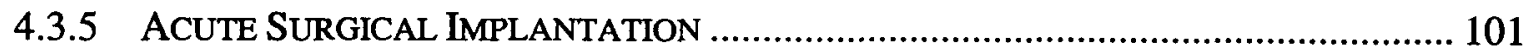

4.4 RESULTS ................................................................................................................ 103

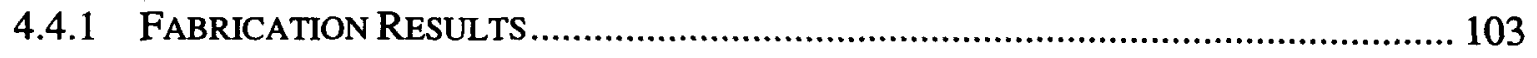

4.4.2 PDMS THICKNESS CHARACTERIZATION RESULTS …….................................... 106

4.4.3 AdHESION TEST AND ELECTRICAL CONTINUITY RESULTS................................... 106

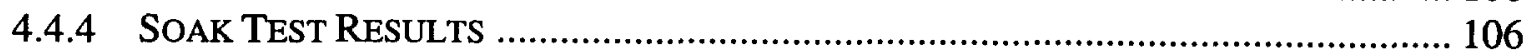

4.4.5 ACUTE SURGICAL IMPLANTATION RESULTS .................................................... 106

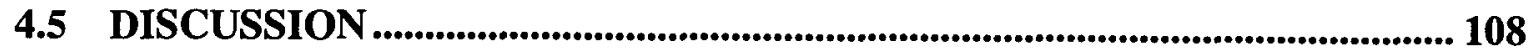

4.6 REFERENCE ................................................................................................................... 110

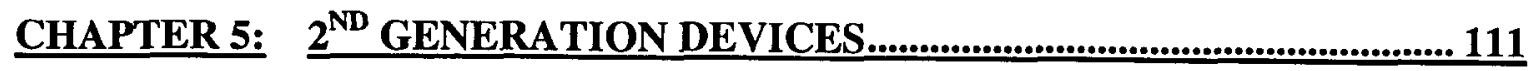

5.1 INTRODUCTION................................................................................................. 111

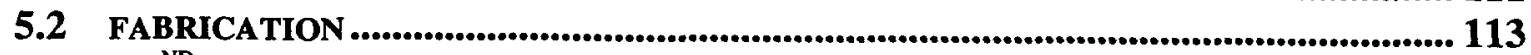

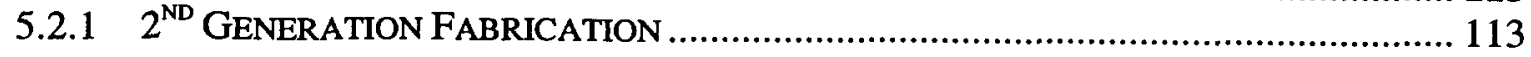


5.3 EXPERMENTAL APPROACH .................................................................................. 116

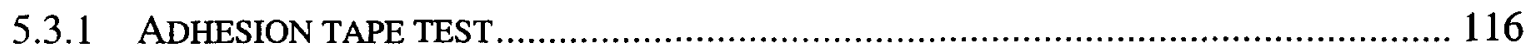

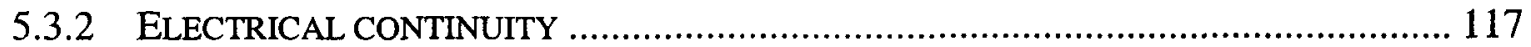

5.3.3 INTERFACING TO ELECTRONICS ……………............................................ 117

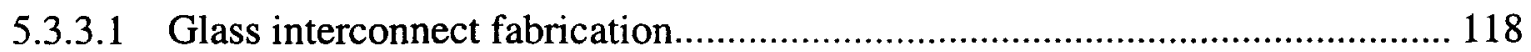

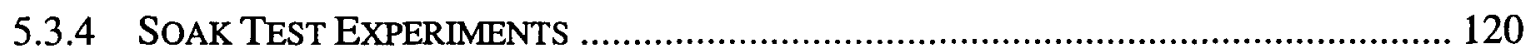

5.3.5 CHRONIC SURGICAL IMPLANTATION EXPERIMENTS .......................................... 121

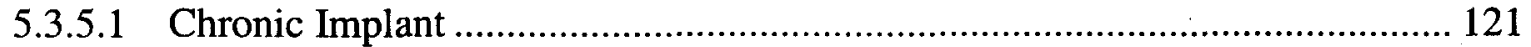

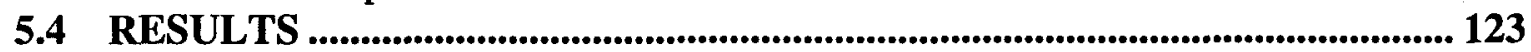

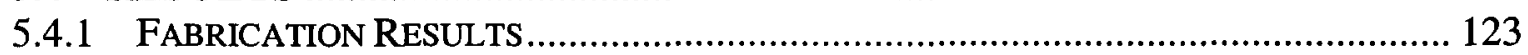

5.4.2 ADHESION AND ELECTRICAL CONTINUITY RESULTS ……............................... 127

5.4.3 ELECTRONIC INTERCONNECT RESULTS ………........................................... 128

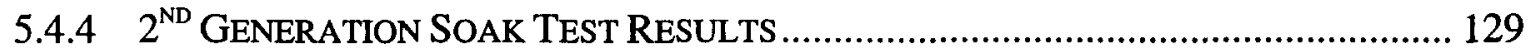

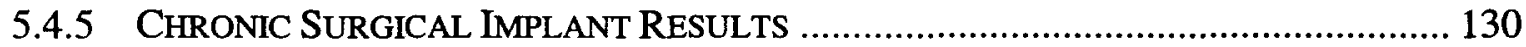

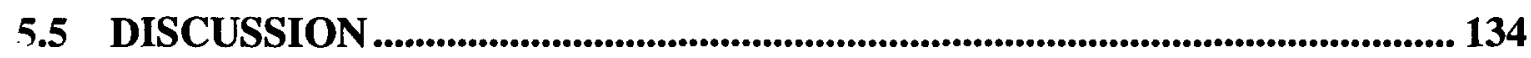

5.6 REFERENCE: ................................................................................................ 137

CHAPTER 6: SURFACE ANALYSIS AND CURING KINETICS ................... 139

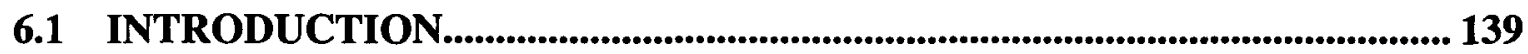

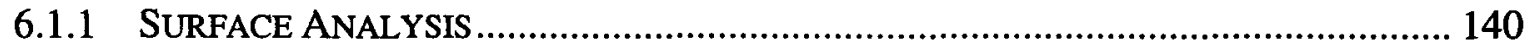

6.1.1.1 Time-of-Flight Secondary Ion Mass Spectrometry ....................................... 140

6.1.1.2 X-ray Photoelectron Spectroscopy .......................................................... 142

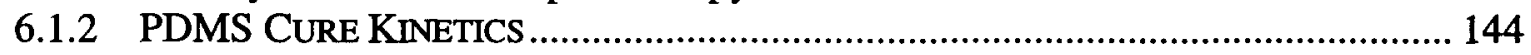

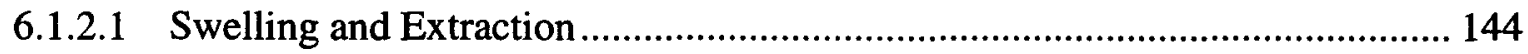

6.1.2.2 Dynamic Mechanical Analysis ................................................................ 145

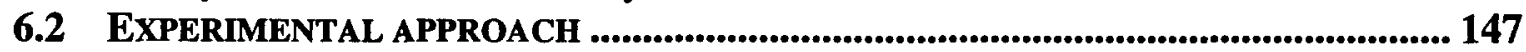

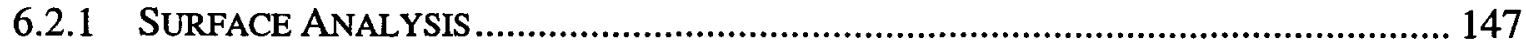

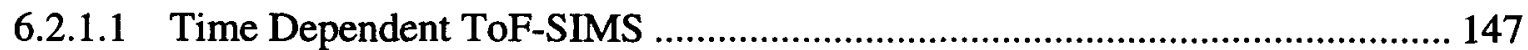

6.2.1.2 Effects of Oxygen Plasma Treatment …………........................................ 147

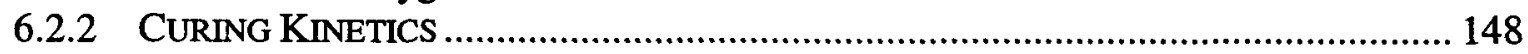

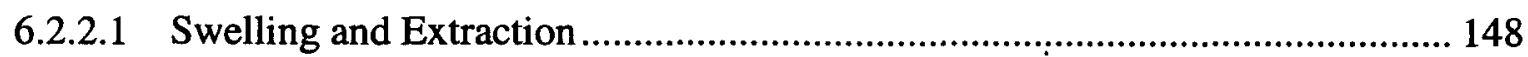

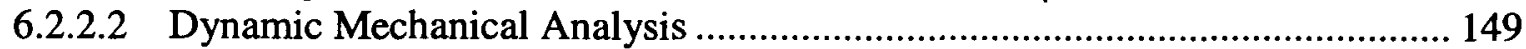

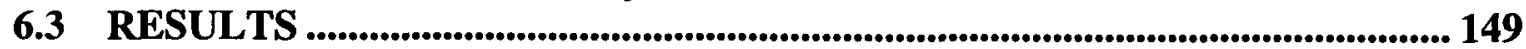

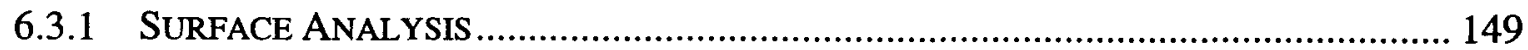

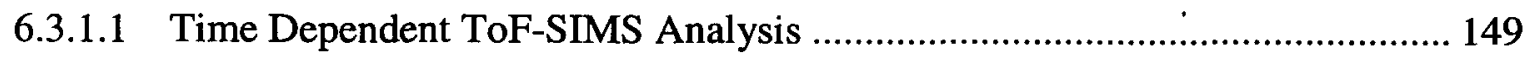

6.3.1.2 Effects of Oxygen Plasma Treatment ………................................................. 153

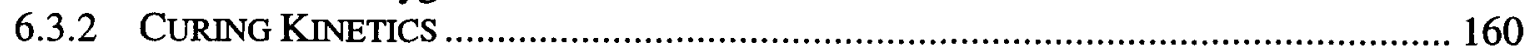

6.3.2.1 Swelling and Extraction Results ................................................................ 160

6.3.2.2 Dynamic Mechanical Analysis ................................................................... 162

6.4 DISCUSSION ................................................................................................... 164

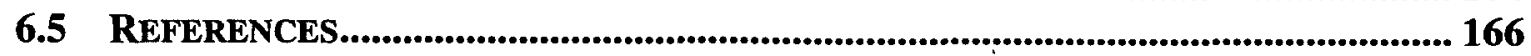




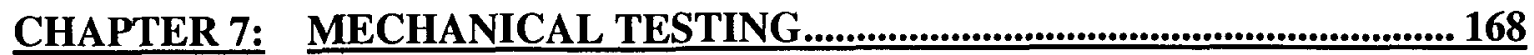

7.1 INTRODUCTION.................................................................................................... 168

7.2 THEORY .......................................................................................................... 169

7.2.1 STRESSES NECESSARY TO WITHSTAND CURVATURE ……................................... 169

7.3 EXPERIMENTAL APPROACH ........................................................................... 171

7.3.1 APPLIED STRESSES............................................................................. 171

7.3.1.1 Sample Preparation for Tensile Testing ........................................................ 171

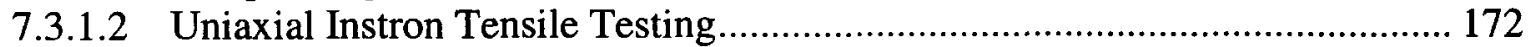

7.3.1.3 Uniaxial Manual Tensile Testing ……………................................................ 173

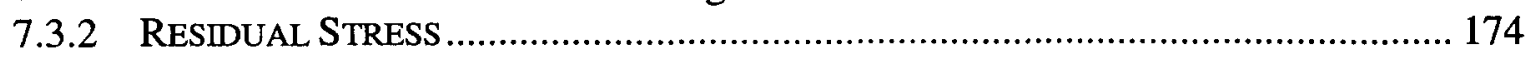

7.3.2.1 PDMS Film on Silicon Substrate .............................................................. 174

7.3.2.2 Metal film on PDMS film ……………..................................................... 176

7.4 RESULTS ............................................................................................................. 177

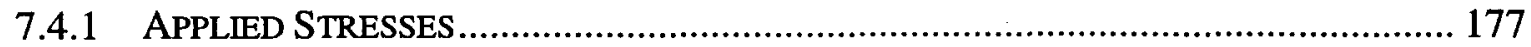

7.4.1.1 Uniaxial Instron Tensile Testing................................................................... 177

7.4.1.2 Uniaxial Manual Tensile Testing .................................................................. 179

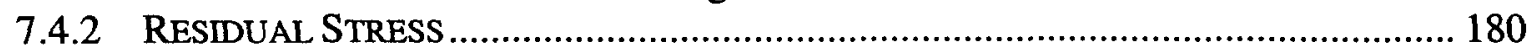

7.4.2.1 PDMS Film on Rigid Substrate ………................................................... 180

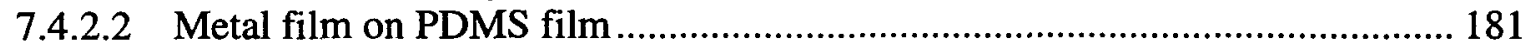

7.5 DISCUSSION ................................................................................................................. 183

7.6 REFERENCES................................................................................................................... 188

CHAPTER 8: CONCLUSION \& FUTURE DIRECTIONS ............................. 190

APPENDIX-1: $\quad$ PDMS THICKNESS CHARACTERIZATION..................194

APPENDIX-2: $\quad 1^{\text {st }}$ GENERATION PROCESS SHEET $\ldots \ldots \ldots \ldots \ldots \ldots \ldots \ldots \ldots \ldots \ldots \ldots \ldots$

APPENDIX-3: $\quad 2^{\text {nd }}$ GENERATION PROCESS SHEET $\ldots \ldots \ldots \ldots \ldots \ldots \ldots \ldots \ldots \ldots \ldots 199$

APPENDIX-4:....GLOSSARY AND ABBREVIATIONS $\ldots \ldots \ldots \ldots \ldots \ldots \ldots \ldots \ldots \ldots \ldots \ldots \ldots 203$ 


\section{OVERVIEW}

Electrical stimulation devices that substitute for malfunctioning sensory neural structures are important bioengineering applications that require integrating microelectronic systems with biological systems. The use of electrical stimulation to recover lost bodily functions has been pursued for over a century; however, the technology necessary to create an implantable electrical stimulation system has been in existence only for a few decades [1-3]. A prime example of such a system is the cardiac pacemaker. This system is comprised of a single stimulation electrode with the circuitry and power supply housed in a rigid titanium canister for protection from biodegradation [4]. Requiring low stimulation frequency and no real-time external control unit, a rechargeable battery is sufficient to power this device. However, a great deal of complexity is added when developing a sensory implant due to the large quantity of information that must be captured, processed, and transmitted in real-time from the surrounding environment to implanted stimulating electrodes. In this case, batteries are no longer a sufficient power supply and must be replaced by a radio frequency (RF) wireless inductive link that transmits both signal and power [5]. In addition to requiring sophisticated data acquisition and power generating components, the size and shape of sensory implants are often dictated by anatomical space constraints. Microtechnology offers a tremendous opportunity to develop microelectronic components capable of interfacing with intricate biological systems. This has been demonstrated by the now commercially available cochlear implants. The success of these devices has motivated several research groups worldwide to investigate the possibility of applying similar technology toward creating the next generation neurostimulation prosthesis for the 
visually impaired. Despite the advancements made during the development of cochlear implants, there are still many challenges to be overcome in order to produce a useful device capable of augmenting an enormously complex system such as vision [6-9].

Age related macular degeneration (ARMD) and retinitis pigmentosa (RP) are amongst the leading causes of blindness, affecting over 10 million people worldwide [10]. These diseases cause blindness through progressive photoreceptor loss in the retina. Genetic engineering and drug therapy aim to retard or stop the course of photoreceptor degeneration; but once photoreceptors are lost, there are limited options to regain vision. Retinal prostheses have great potential in alleviating the problems and disabilities produced by ARMD and RP. Lawrence Livermore National Laboratory is part of a multidisplinary epiretinal prosthesis research team whose goal is to create "a high-density microelectronic tissue hybrid sensor for imaging" [11]. This consortium is sponsored by the Department of Energy's (DOE) Office of Biological and Environmental Research (OBER) and consists of five national laboratories, three universities, and one private company. The DOE retinal prosthesis team is exploring the possibility of using an intraocular microelectronic device that would restore some vision to patients with retinal disease, specifically ARMD and RP. As a replacement for the non-functional lightsensitive cells, the device will receive information about the visual scene using an attached camera. This information will be transformed into suitable electrical impulses capable of exciting the still viable inner retinal cells, thus resulting in vision recovery. A schematic illustration of this concept is shown in Fig. 0.1. 


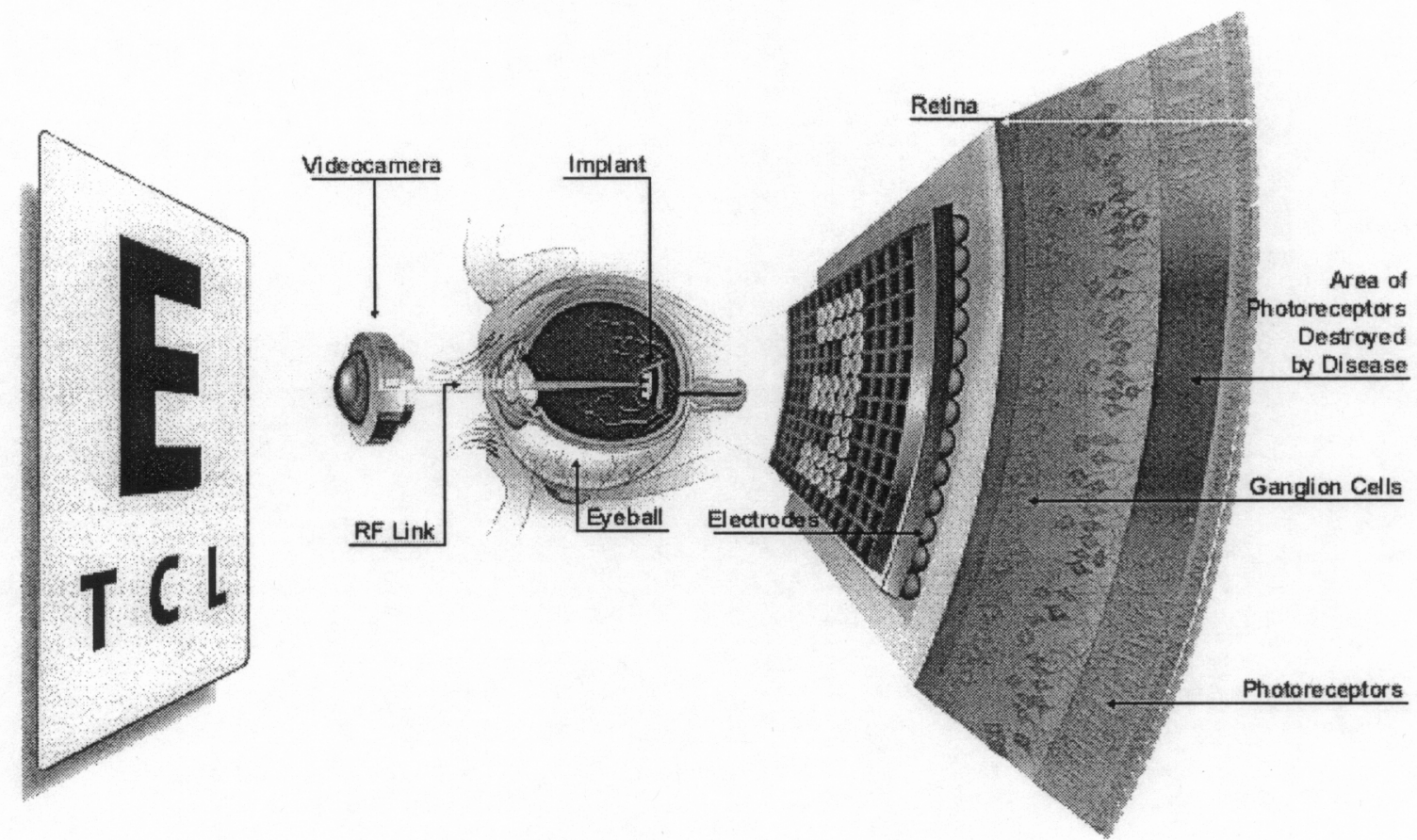

Figure 0.1. Diagram of a retinal prosthesis utilizing a video camera and RF link to send images to a microelectrode array where they are converted into electrical signals and stimulate the retina. Used with permission from Dr. Mark Humayun [11, 12].

The epiretinal prosthesis project is presented with significant technical challenges. It requires an inter-disciplinary approach to develop several subsystems that perform distinct functions. These include units for image acquisition, image-processing electronics, a high-bandwidth telemetry system to provide power and data in real-time to the implanted subsystems, implanted electronics for signal decoding and a high-density microelectrode array for delivery of charge to the retina. Once these complex subsystems are developed they must be integrated in order to produce a functional prosthesis. Recent advancements in microtechnology, integrated circuits, and wireless communication provide the prospect to produce such a complex system [2, 6, 8, 13-15]. As part of this multi-disciplinary epiretinal prosthesis consortium, LLNL's role is to develop the highdensity implantable microelectrode array component of the prosthesis. This array will 
serve as the interface between an electronic imaging system and the human eye, directly stimulating retinal neurons via thin film conducting traces. This dissertation presents the development of a scaleable silicone-based microfabrication platform for producing an implantable microelectrode array.

Advancements in microfabrication technology provide the background necessary to conceptualize an intricate, active electronic device small enough to be implanted in the eye. Nevertheless, the production of a high-density implantable microelectrode array still entails a broad range of development efforts, which are addressed in this dissertation. These efforts include selection of materials, development of fabrication processes, electronic interfacing, and packaging. Since the device is intended as a long-term implant that interfaces with delicate retinal cells, vital biological and physical design requirements must be met. The device is required to: (1) conform to the curvature of the retina without inducing detrimental stress, (2) be flexible and robust to withstand handling during fabrication and implantation, (3) be intraocularly biocompatible for permanent implantation, and (4) be capable of interfacing to an integrated circuit (IC) chip and supporting electronics to receive power and data wirelessly to allow for complete system integration.

The size specification of the microelectrode array is confined to $16 \mathrm{~mm}^{2}$ (4 X 4 $\mathrm{mm}$ ) to minimize stress and surgical trauma [7]. Despite the small size, the implant will also need to contain 1000 electrodes along with a tack hole for anchoring the device. It has been shown that the greater the number of electrodes, the higher the resolution of the image [2]. Producing an implant that contains one thousand electrodes in a $4 X 4 \mathrm{~mm}$ area is challenging, but achievable with scaleable microfabrication technology. To 
accomplish this, the electrode size must be large enough to deliver the necessary charge $(1 \mu \mathrm{C})$ to induce visual percept, while minimizing the production of toxic by-products [16]. While balanced biphasic pulses help to reverse any toxic reaction, charge density must be minimized by increasing electrode surface area. In addition, the electrode material must be biocompatible, inert, corrosion-resistant, and possess a high charge injection capability. Platinum $(\mathrm{Pt})$ and iridium oxide $\left(\mathrm{IrO}_{2}\right)$ are the prime candidates for the electrode material since they have been proven to exhibit most of these characteristics [17].

The potential for damage to the delicate retina during abrupt eye movement may indicate the need to use thin, highly conformable soft substrate materials for an implanted array. Foreseeing the incompatibilities of conventional microfabrication materials, such as silicon and glass, polymer-based technologies are currently being pursued $[4,18,19]$. Although polymers such as polyimide have well-established microfabrication processing technology history, they lack the conformability and softness offered by silicone rubbers. In this project, a type of silicone rubber, poly(dimethyl siloxane), was determined to be a suitable substrate for fabricating the implantable microelectrode array $[18,20]$. The selection of silicone rubber mandated the enhancement of currently available polymerbased fabrication techniques and the development of new microfabrication processes.

We have proposed that silicone-based microfabrication processes will be required to enable the development of an implantable microelectrode array for an epiretinal prosthesis. To demonstrate this, several feasibility prototypes were developed and tested. These prototypes consisted of an exposed electrode region with patterned metal traces for electrical contact sandwiched between thin silicone rubber layers (total thickness $\sim 50$ 
$\mu \mathrm{m})$. Applying the sophistication of microfabrication techniques to biocompatible polymeric materials provides an exciting opportunity of translating the concept of retinal prosthesis into an implantable reality.

The following section summarizes the topics covered in this dissertation. Figure 0.2 provides a project overview flow chart with the emphasis of this dissertation highlighted in red.

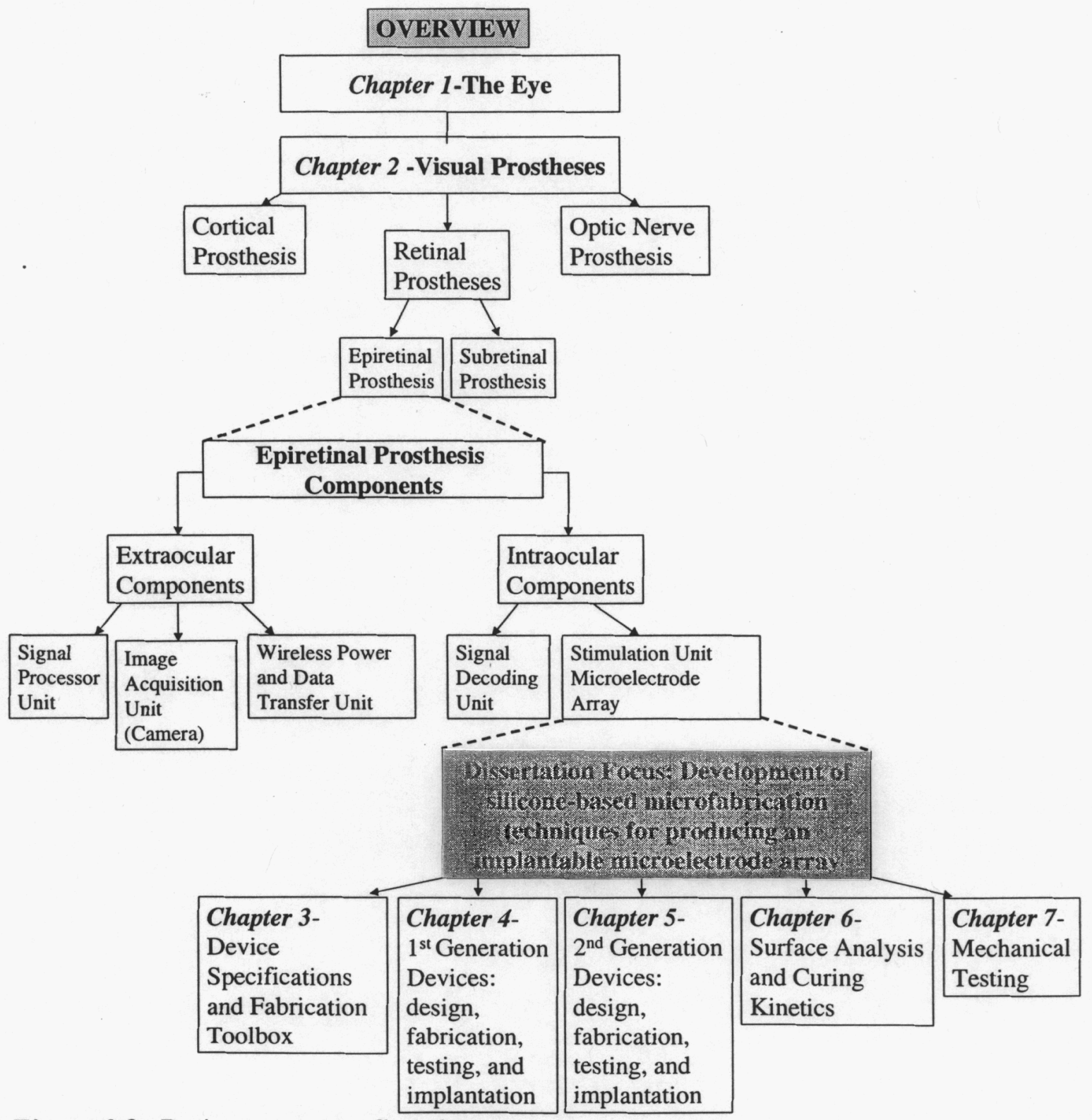

Figure 0.2. Project overview flowchart 


\section{CHAPTER 1: The Eye}

The major causes of blindness are degenerative diseases of the retina. The most common of these are age-related macular degeneration and retinitis Pigmentosa; with both targeting photoreceptors. In order to design a suitable retinal implant, one needs to understand the structure and function of the eye, and more specifically, the retina.

\section{CHAPTER 2: Visual Prostheses}

This chapter provides relevant background on visual prostheses. The ultimate objective of any artificial visual aid is to capture visual data and transmit this information in real-time to the upper-level visual processing center. Researchers have made tremendous progress on the path to producing a viable implant to restore vision to some blind patients.

\section{CHAPTER 3: Device Specifications and Fabrication Toolbox}

This chapter addresses the microelectrode array device specifications and the fabrication toolbox that is used to meet these specifications. The fabrication methods described focus on the exploitation of the physical and chemical properties of the polymer PDMS for the microelectrode array development. PDMS offers many attractive characteristics including conformability, biocompatibility, low temperature processing and the ability to bond to itself as well as other materials.

\section{CHAPTER 4: $1^{\text {st }}$ Generation Devices}

This chapter addresses the $1^{\text {st }}$ generation devices, which were designed, fabricated, and acutely implanted in a canine eye. Initial device handling was somewhat difficult, due to the tendency of the thin $(\sim 50 \mu \mathrm{m})$ devices to curl. However, the PDMS 
implant appeared to conform to the retina. Forceps used to maneuver the device damaged peripheral metal traces during implantation.

\section{CHAPTER 5: $2^{\text {nd }}$ Generation Devices}

This chapter presents the $2^{\text {nd }}$ generation devices. Based on the $1^{\text {st }}$ generation results, $2^{\text {nd }}$ generation devices were designed, fabricated, and chronically implanted into a canine eye. Of the most significant modifications made were counter measures to prevent surface contamination and passivation problems; increasing fabrication device yield by approximately $300 \%$. Another vital modification made was the inclusion of micro-molded ribs around the device perimeter, which facilitated handling, implantation and reinforced the electrical traces near the edges.

\section{CHAPTER 6: Surface Analysis and Curing Kinetics}

This chapter explores how material characteristics can be tailored to develop reliable and repeatable fabrication processes. Surface treatments and PDMS cure time critically impacted the general performance of PDMS and thus affected the fabrication processes.

\section{CHAPTER 7: Mechanical Stresses}

Robust microelectrode arrays are prerequisite as stretching occurs during fabrication and implantation. Mechanical testing indicated that design and fabrication parameters of metal traces significantly affect stretchablity of the devices. Both applied and residual stresses are addressed

\section{CHAPTER 8: Conclusion and Future Directions}

The use of a PDMS substrate for producing an implantable microelectrode array required that new fabrication techniques be developed. Multiple process iterations were 
required to establish the most appropriate process flow. The work presented in this dissertation provides a silicone-based fabrication platform for the development of the high-density microelectrode array. While experiments to date using this new platform technology have all been promising, there is still substantial research effort required to produce a fully functional implant.

\section{REFERENCES}

[1] G. S. Brindley, "The site of electrical excitation of the human eye," J. Physiol., vol. 127, pp. 189-200, 1955.

[2] M. Humayun, "Intraocular Retinal Prosthesis," Tr. Am. Ophth. Soc, vol. 99, pp. 271-300, 2001.

[3] M. Clements, K. Vichienchom, W. T. Liu, C. Hughes, E. McGucken, C. DeMarco, J. Mueller, M. Humayun, E. de Juan, J. D. Weiland, and R. Greenberg, "An implantable neuro-stimulator device for a retinal prosthesis," International Solid-state circuits conference, pp. 216-17, 1999.

[4] J. U. Meyer, T. Stieglitz, O. Scholz, W. Haberer, and H. Beutel, "High density interconnects and flexible hybrid assemblies for active biomedical implants," IEEE Transactions on advanced packaging, vol. 24, pp. 366-374, 2001.

[5] T. Bell, K. D. Wise, and D. J. Anderson, "A flexible micromachined electrode array for a cochlear prosthesis," Sensors and Actuators A Physical, vol. A66, pp. 63-69, 1998.

[6] E. Zrenner, "Will retinal implants restore vision?," Science, vol. 295, pp. 1022$1025,2002$.

[7] M. Humayun and E. de Juan, "Artificial vision," Eye, vol. 12, pp. 605-7, 1998.

[8] J. U. Meyer, "Retina implant: a bioMEMS challenge," Sensors and Actuators A Physical, vol. 97-98, pp. 1-9, 2002.

[9] D. Pruves, G. J. Augustine, D. Fitzpatrick, K. C. Lawrence, A. S. LaMantia, and J. O. McNamara, "Neuroscience." Sunderland: Sinauer Associates, Inc., 1997. 
[10] "Statistics on blindness and blinding diseases in the United States," The University of Washington Department of Ophthalmology, 2002.

[11] DOE, "A High-Density Microelectronic-Tissue Hybrid Sensor for Imaging," DOE LAB 01-14 Biomedical Engineering Program, 2001.

[12] M. Humayun, E. de Juan, G. Dangnelie, R. Greenberg, R. Propst, and P. DH, "Visual perception elicited by electrical stimulation of retina in blind humans," Arch. Ophthalmol, vol. 114, pp. 40-6, 1996.

[13] Q. Bai and K. D. Wise, "Single-unit neural recording with active microelectrode arrays," IEEE Transactions on Biomedical Engineering, vol. 48, pp. 911-920, 2001.

[14] L. Griscom, P. Degenaar, B. LePioufle, E. Tamiya, and H. Fujita, "Techniques for patterning and guidance of primary culture neurons on micro-electrode arrays," 2002.

[15] W. T. Liu, K. Vichienchom, M. Clements, C. DeMarco, C. Hughes, E. McGucken, M. Humayun, E. de Juan, J. D. Weiland, and R. Greenberg, "A neurostimulus chip with telemetry unit for retinal prosthetic device," IEEE Journal of Solid-State Circuits, vol. 35, pp. 1487-97, 2000.

[16] A. Hung, D. Zhou, R. Greenberg, and J. Judy, "Micromachined Electrodes for High Density Neural Stimulation Systems," presented at IEEE special topics in medicine and biology, Madison, WI, 2002.

[17] W. T. Liu and M. Humayun, "Artificial Retinal Prosthesis to Restore Vision for the Blind," presented at Digest of the LEOS Summer Topical Meetings, 2000.

[18] P. Krulevitch, W. Bennett, J. Hamilton, M. Maghribi, and K. Rose, "Polymerbased packaging platform for hybrid microfluidic systems," Biomedical Microdevices, vol. 4, pp. 301-308, 2002.

[19] M. Maghribi, J. Hamilton, D. Polla, K. Rose, T. Wilson, and P. Krulevitch, "Stretchable Micro-Electrode Array for Retinal Prosthesis," presented at IEEEEMBS special topics n medicine and biology, Madison, WI, 2002. 
[20] F. H. Silver and D. L. Christiansen, Biomaterials Science and Biocompatibility. New York: Springer-Verlag, 1999. 


\section{CHAPTER 1: THE EYE}

\subsection{INTRODUCTION}

Age Related Macular Degeneration (ARMD) and Retinitis Pigmentosa (RP), among the leading causes of blindness [1], affect over 10 million people worldwide through progressive photoreceptor loss in the retina [2]. The photoreceptor cells in a healthy retina initiate electrochemical signals in response to incident light. This neural signal is further processed by bipolar and ganglion cells of the inner retina prior to final processing by the visual cortex in the brain [3]. Photoreceptors in the outer retina are almost completely absent in the retina of end-stage ARMD and RP. However, bipolar cell and ganglion cells in the inner retina, through which the photoreceptors normally synapse, survive at higher rates in the macular region $[4,5]$, but to a lesser extent beyond the macula [6]. Since the ganglion and bipolar cells remain intact, they are the target cells to be artificially induced by electrical stimulation.

Vision allows us to know more about our environment than any of the other four senses. The eye, the functional organ of vision, is the link between objects in our world and the electrical impulses that are interpreted as visual images by our brains. Our eyes capture light from the surroundings and transform it into nerve impulses, which are then interpreted by the brain. The eye cannot function in the absence of light, yet it can in full or dim light [7]. It can interpret color, size, shape, and depth by imaging the light that is reflected off objects around us [7]. In designing an implant to be used as a retinal prosthesis, it is important to understand the anatomy and physiology of the eye as well as the diseases that lead to the need for visual prostheses. 


\subsubsection{Basic function of the eye}

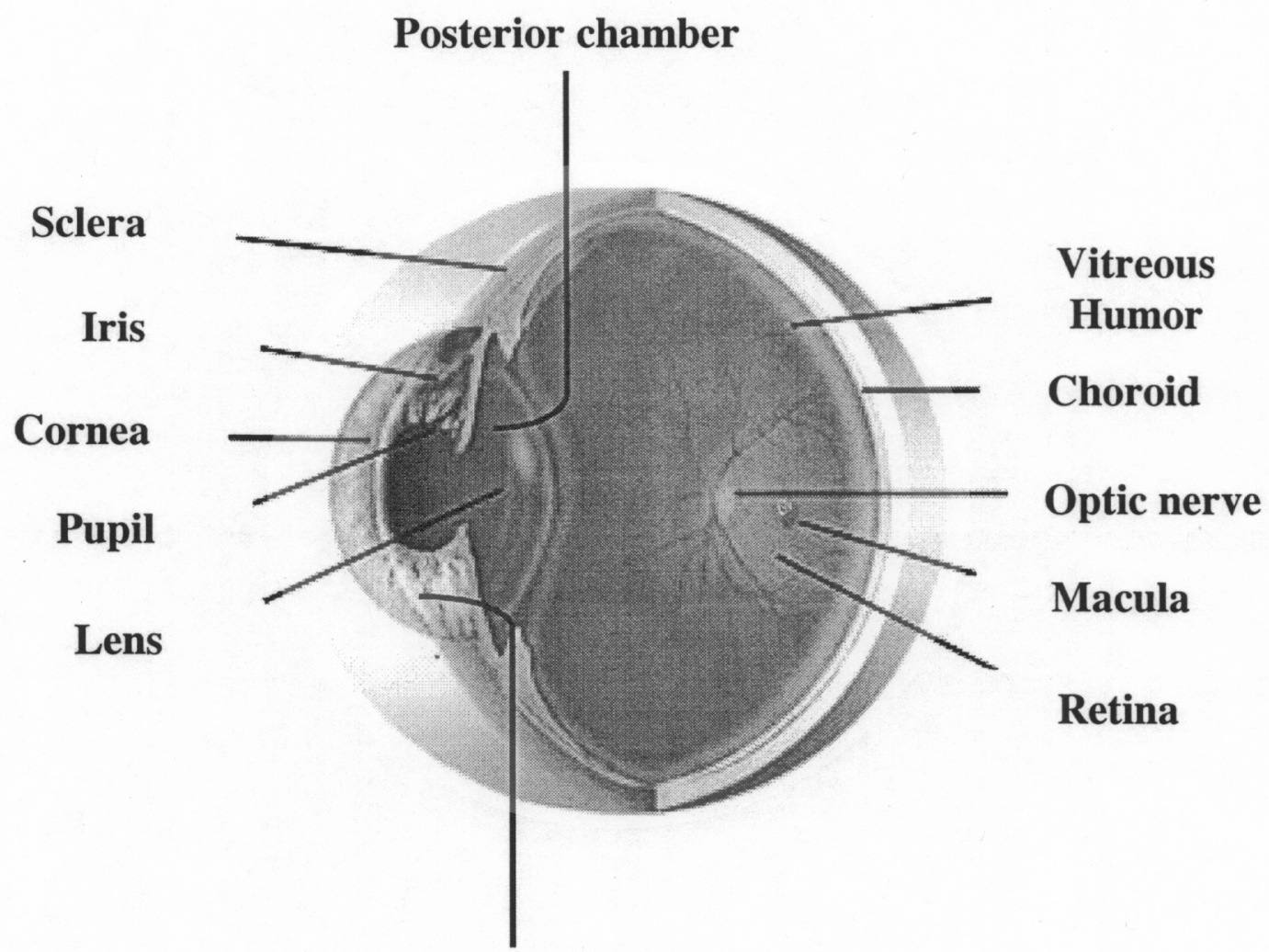

Anterior chamber

Figure 1.1. Anatomy of the eye adapted from [8]

The human eye is roughly spherical with an axial length of approximately $24 \mathrm{~mm}$ on average [3]. The bony tissues of the face, which surround it, are referred to as the orbit [7]. The eye rests in the front half of the orbit upon a muscular hammock surrounded by adipose and connective tissue [9]. The eye is steered by a set of six muscles [9]. These muscles are activated by nerves, which, along with several key veins and arteries, enter the orbit behind the eye through an opening from the rear called the optic foramen [7]. There are three main compartments of the eye as seen in Fig. 1.1. The outermost compartment is called the anterior chamber; it is comprised of the cornea in the front and the iris or colored section on the inside [7]. The cornea is a clear convex 
window that is integrated onto the main sphere of the eye comprised of the sclera, which is the white region of the eye. The junction of where the cornea meets the sclera is the limbus [7]. The second compartment is the cavity immediately behind the iris and in front of the lens, and is called the posterior chamber [7]. Both the anterior and posterior chambers are filled with a watery liquid, called aqueous humor, which percolates through the eye, providing nourishment and cleansing [10]. The third and largest compartment is the cavity of the vitreous. A gelatinous substance, called the vitreous humor, occupies this space and maintains the structure of the eye [7], as seen in Fig. 1.1.

The eye is a complex optical system and the ability to see is dependent on the actions of several structures in and around the eyeball. When one looks at an object, light rays are reflected from the object to the cornea [7]. From there, the light travels through clear aqueous fluid, and passes through a small aperture called the pupil. As muscles in the iris relax or constrict, the pupil changes size to adjust the amount of light entering the eye [7]. Light rays are focused through the crystalline lens. The sharpness of the final picture is adjusted automatically by changing the shape, and thereby the refractivity, of the crystalline lens. This focusing system is so powerful that the light rays intersect at a point just behind the lens inside the vitreous humor and diverge from that point back to the retina [7]. The resulting image on the retina is upside-down. Here at the retina, the light rays are converted to electrical impulses. These impulses are then transmitted through the optic nerve, an electrochemical conduit, connecting the eyeball to the brain [7]. From there, optic radiations extend to the visual cortex in the occipital lobe of the brain toward the visual cortex [3] where the image is translated and perceived in an upright position. An overview of this pathway is illustrated in Fig. 1.2. 
Like a camera, the eye consists of a lens and a recording medium to produce an image. The eyeball's lens is compromised of the cornea, crystalline lens, and vitreous to refract and focus the light, and a film consisting of the retina on which the rays are focused. If any one or more of the lens components is not functioning properly, the result is refractive problems and poor focusing of images on the retina. If the retina is not functioning properly then more dramatic consequences may result, including blindness.
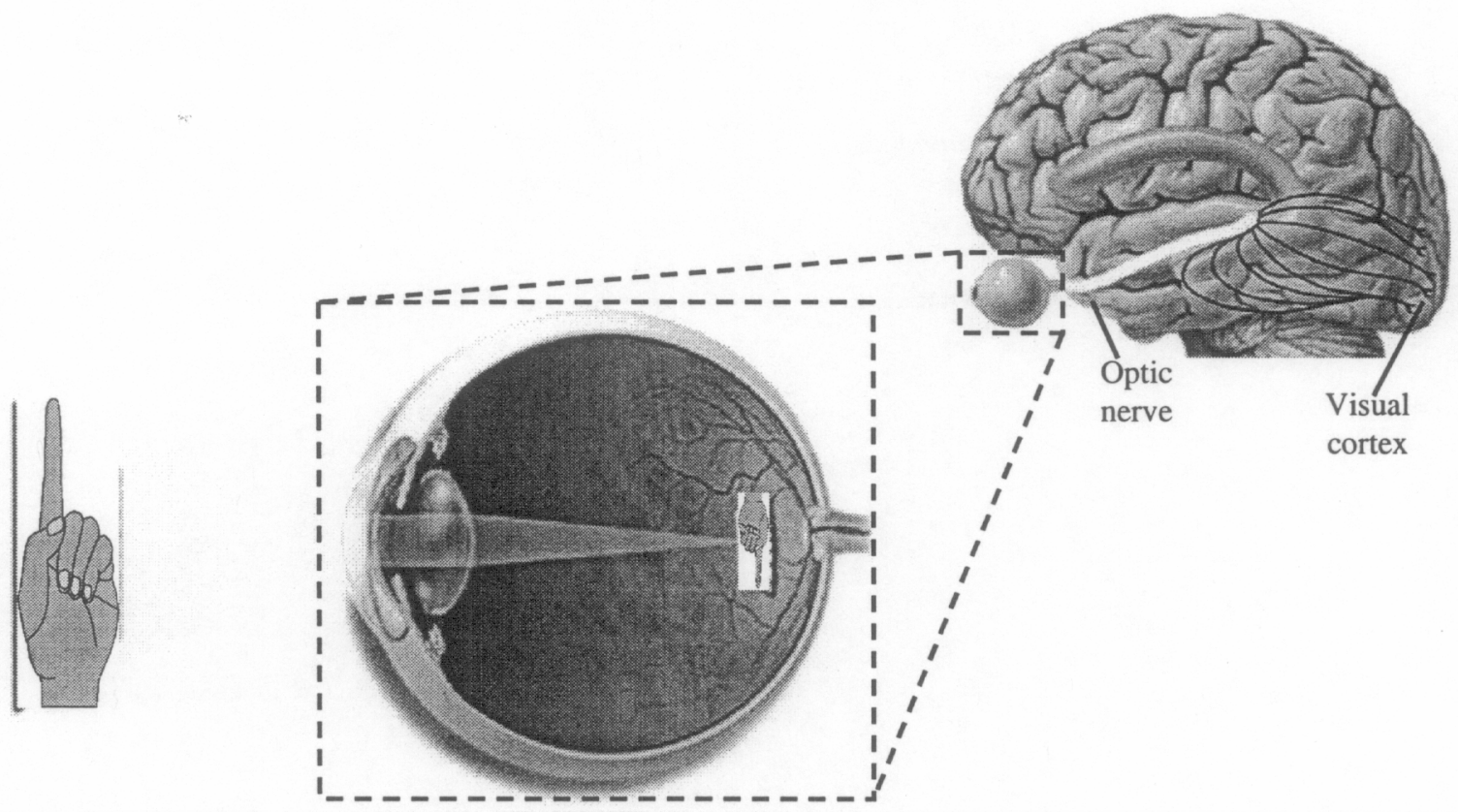

Figure 1.2. Overview of the visual pathway adapted from $[8,11]$

\subsection{THE RETINA}

The retina is a thin $(0.25 \mathrm{~mm})$ delicate neural tissue [7], which senses the light entering the eye. It converts this light information into neural electrical signals. An image of a healthy retina is shown in Fig. 1.3. It is the innermost layer lining the inside of the eye opposite the crystalline lens and is comprised of hundreds of millions of nerves distributed within its layers, which also contain the vessels and photochemical receptors necessary for vision [7]. 


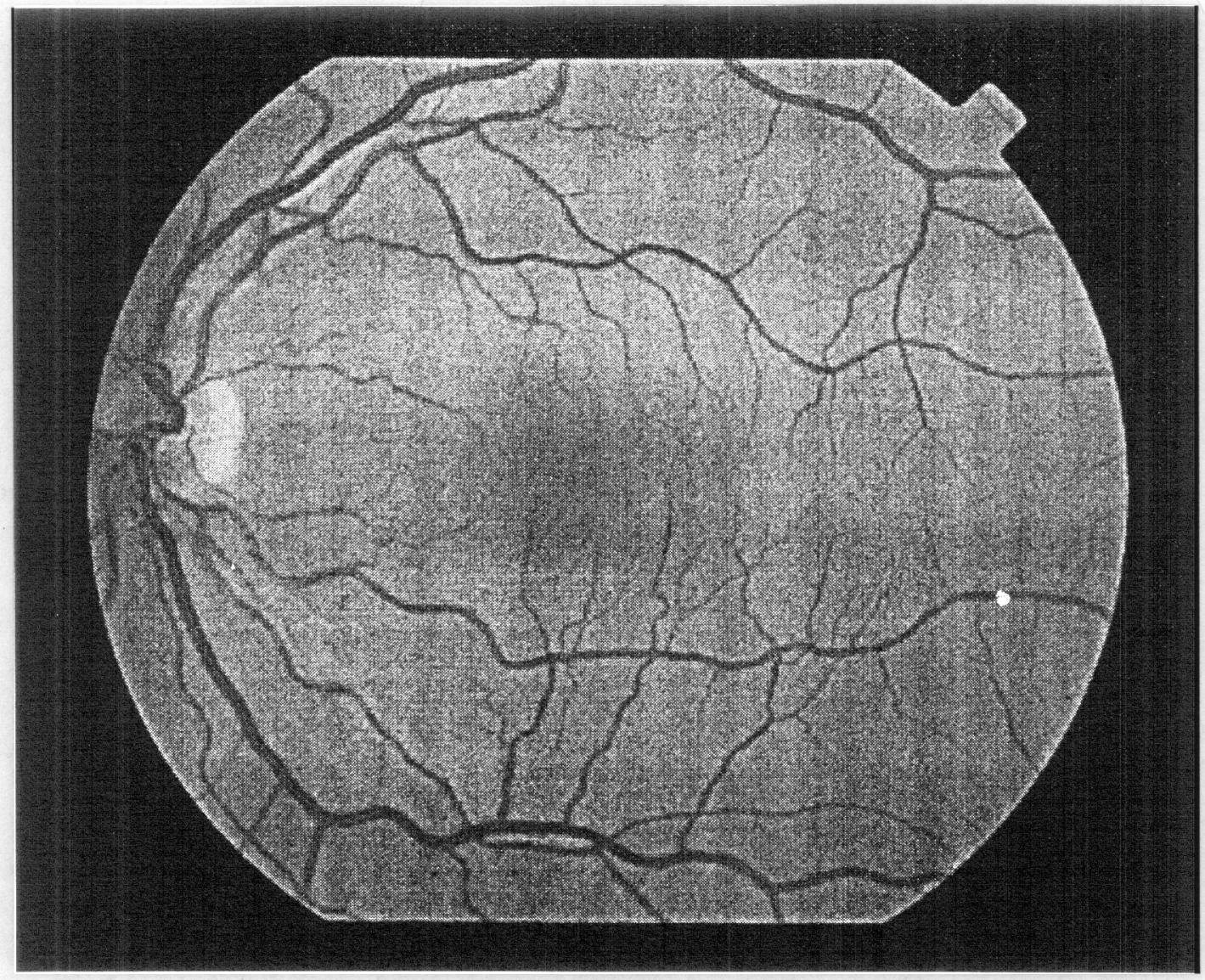

Figure 1.3. Image of a healthy retina used with permission from Dr. Mark Humayun

The retina is composed of approximately 126 million photoreceptors and one million ganglion cells [7]. The axons of the ganglion cells form the optic nerve, which extends into the visual cortex of the brain. There are many millions of interneurons packed into the retina intervening between the photoreceptors and the ganglion cells. Signal processing and image convergence is performed in all neural cell layers involving bipolar cells, horizontal cells, amacrine cells and ganglion cells [3], as seen in Fig. 1.4. The photoreceptors provide an analog graded potential to the attached bipolar neural cell layer [3]. The bipolar cells relay the spatial-temporal information to the ganglion cells. 
While horizontal cells and amacrine cells are responsible for the lateral interactions within the retina [12].

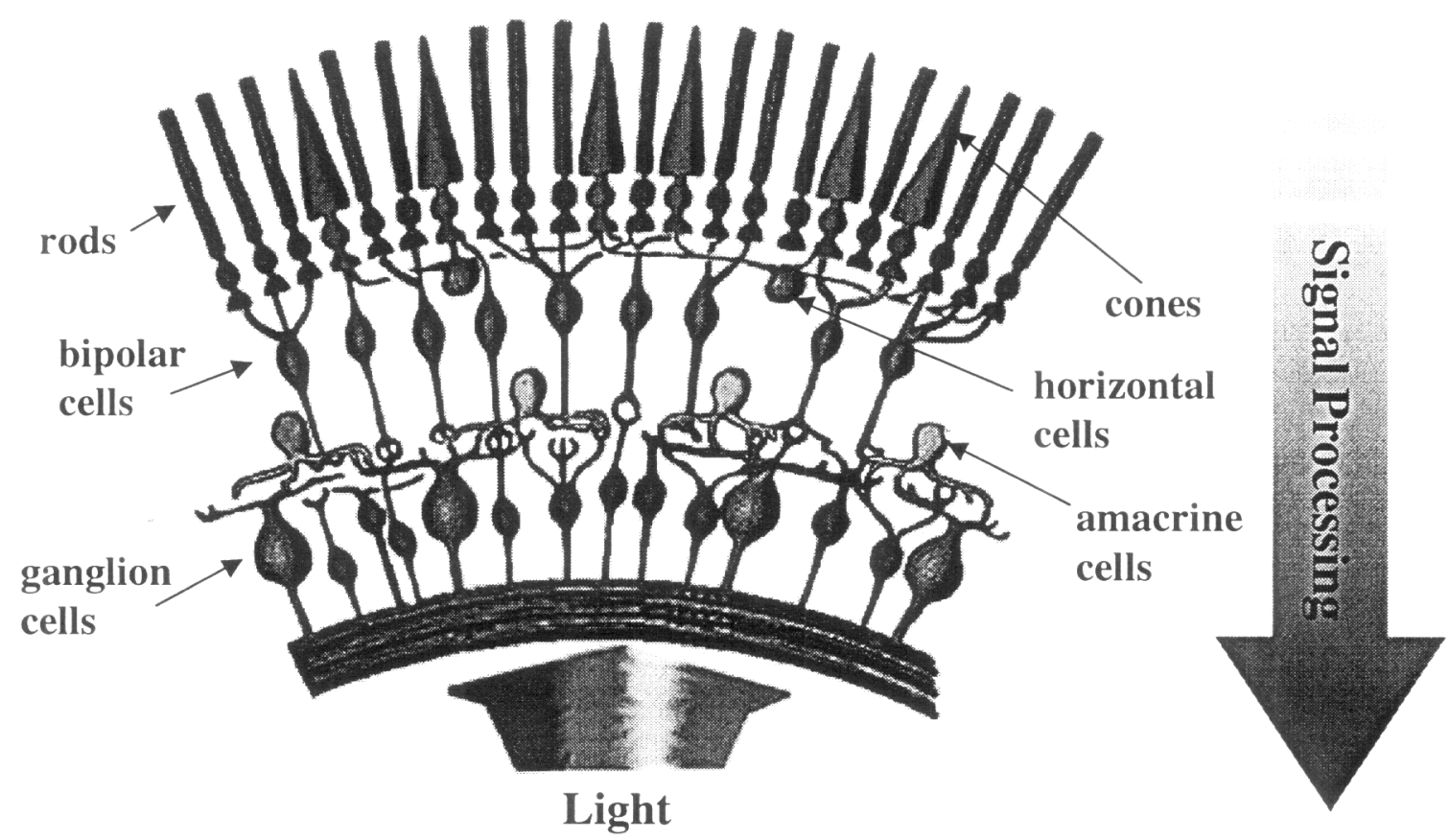

Figure 1.4. Illustration of retinal sensory photoreceptors and the ganglion cells with connecting cell types showing light and signal pathways, adapted from [13].

The retina contains two types of photoreceptor cells, which are referred to as the "rods" and the "cones" because of their respective anatomical shape. The rods are concentrated outside the center of the retina and are required for peripheral vision and for night vision. The largest numbers of receptors are the rods, estimated to be approximately 120 million per eye [7]. The rods function best in low illumination, reaching maximum sensitivity after being in the dark for approximately 30 minutes [7]. This dark adaptation explains the inability to see when first entering a dark room, but also explains why one can see much better after the passage of time. The rods cannot distinguish color; they are black-and-white receptors [14]. 
The cones are concentrated in the central part of the retina, known as the macula and are responsible for detail and color vision. Cone cells work best in high illumination. The macula is located in the central retina directly behind the pupil. The tiny, central portion of the macula is referred to as the fovea and is responsible for our fine detail vision. The center of the retina has a 1:1 ratio between the photoreceptors and ganglion cells; the far periphery has a ratio of $300: 1$ [14]. This results in a gradual shift in resolution from central vision to peripheral vision.

\subsubsection{Light Pathway In The Retina}

The retina is a seven-layered structure responsible for signal transduction [7]. Light enters from the ganglion cell side, and must penetrate all cell types before reaching the rod and cone photoreceptors. The outer segments of the rods and cones then transduce the light and send the signal through the cell bodies of the outer nuclear layer and out to their axons $[7,14]$. From there the signal moves to the outer plexiform layer axons, which contact the dendrites of bipolar cells and horizontal cells. Horizontal cells are interneurons, which aid in signal processing. The bipolar cells in the inner nuclear layer process input from photoreceptors and horizontal cells, and transmit the signal to their axons [7]. In the inner plexiform layer, bipolar axons contact ganglion cell dendrites and amacrine cells, another class of interneurons [7]. The ganglion cells penetrate their axons through the nerve fiber layer to the optic disk to make up the optic nerve.

\subsubsection{Action Potentials}


It is important to understand how neurons function in order to appreciate the complexity of the natural biological process of vision. Neurons communicate through an electrochemical process, which means that a chemical flux across the cellular membrane results in an electrical signal. When a neuron is not sending a signal, it is said to be at rest and the internal charge of the neuron is negative relative to the outside. Although the concentrations of the different ions attempt to balance out on both sides of the membrane, they are unable to because the cell membrane allows only some ions to pass through to maintain the cell's rest state. At rest, potassium ions $\left(\mathrm{K}^{+}\right)$can cross through the membrane easily [12], while chloride ions $\left(\mathrm{Cl}^{-}\right)$and sodium ions $\left(\mathrm{Na}^{+}\right)$have a more difficult time crossing [12]. In addition to these selective ion channels, cell membranes contain sodium-potassium pumps that consume energy to move three sodium ions out of the neuron for every two potassium ions it puts in. When all these forces balance out, and the difference in the voltage between the inside and outside of the neuron is measured, the resting potential is approximately $-70 \mathrm{mV}$. At rest, there are relatively more sodium ions outside the neuron and more potassium ions inside the neuron.

The action potential occurs when the neuron is excited. The action potential is an explosion of electrical activity that is created by a depolarizing current. This means that a stimulus causes the resting potential to move toward $0 \mathrm{mV}$ [12]. When the depolarization reaches the threshold of $-55 \mathrm{mV}$, a neuron fires an action potential [12]. If the neuron does not reach this critical threshold level, then no action potential will fire. The action potential size is not governed by the strength of the stimulus once the threshold level is reached; the size of the action potential is always the same. Therefore, the neuron either does not reach the threshold or a complete action potential is fired; this is an all or none 
principle. The cause of the action potential is an exchange of ions across the neuron membrane. A stimulus first results in the opening of sodium channels. Because there are more sodium ions on the outside, and the inside of the neuron is negative relative to the outside, sodium ions rush into the neuron. Since sodium has a positive charge, the neuron becomes more positive and becomes depolarized. It takes longer for potassium channels to open. When they do open, potassium rushes out of the cell, reversing the depolarization. Also at about this time, sodium channels start to close. This causes the action potential to go back toward $-70 \mathrm{mV}$, which is referred to as repolarization [12].

In most sensory systems, activation of a receptor by the appropriate stimulus causes depolarization to trigger an action potential as explained above. However in the visual system the process is somewhat reversed, although the principle is the same. Shining light on to the photoreceptors results in hyperpolarization rather than depolarization. Hyperpolarization means that the potential goes in the negative direction from the resting potential. It turns out that in the dark the photoreceptors are depolarized with membrane potential of approximately $-40 \mathrm{mV}$ [12]. An increase in illumination causes the potential across the receptor to become more negative and saturates at $-65 \mathrm{mV}$ [12]. The reversal of events for the photoreceptors versus other sensory systems is not completely understood.

\subsubsection{Retinal Signal Processing}

A photoreceptor is hyperpolarized whenever photons strike it. On the other hand, a ganglion cell is more selective and only responds to a small spot of light, a small ring of light, or a light-dark edge; but not to diffuse light. The behavior of the ganglion cell is 
referred to as a center-surround receptive field [14]. This means that the cell will respond when the center is mainly light and the surround mainly dark, or vice versa. This complex receptive field is created by the interneurons of the retina: the bipolar cells and the horizontal cells, primarily.

Diffuse light hyperpolarizes the cone or rod. The cone thereby excites the bipolar cell, which then excites its ganglion cell. Meanwhile, the same events are happening to neighbor cells. However, the neighbor cones also excite horizontal cells. The horizontal cells send processes laterally and inhibit the center bipolar cells [12]. A small spot of light, however, excites the bipolar cell but not its neighbors. There is no inhibition, leading to an excitation of the ganglion cell. A ring of light excites only the neighbors $[12,15]$. Now, the bipolar cell is strongly inhibited, with no excitation. In response to this strong silencing of the bipolar cell, the ganglion cell shuts down as well. This is a brief glimpse into the complexity of the visual system, which becomes even more elaborate as the chain of events move to the visual cortex [12]. In summary, the entire visual system exists to see the world as a pattern of lines, even things as complex as a face. Colors and brightness are judged by comparison, not by any absolute scale. This system of lateral inhibition in the retina is the first step towards sharpening contours and picking up on borders between light and dark [14]. The ganglion cell ignores diffuse light, but a sharp dot will result in cell excitation. Higher up in the cortex, all these dots will be combined into lines, which will be combined into curves making out objects.

\subsection{PHOTORECEPTORS (CONES AND RODS)}


Light causes a chemical reaction with "iodopsin" in cones and with "rhodopsin" in rods, beginning the visual process. As described previously, activated photoreceptors stimulate bipolar cells, which in turn stimulate ganglion cells. There are about 6.5 to 7 million cones in each eye, and they are sensitive to bright light and to color. The highest concentration of cones is in the macula. The fovea centralis, at the center of the macula, contains only cones and no rods. There are 3 types of cone pigments, each most sensitive to a certain wavelength of light. The wavelength of light perceived as brightest to the human eye is $555 \mathrm{~nm}$, a greenish-yellow [14].

There are about 120 to 130 million rods in each eye, and they are sensitive to dim light, to movement, and to shapes [14]. The highest concentration of rods is in the peripheral retina, decreasing in density up to the macula. Rods do not detect color, which is the main reason it is difficult to tell the color of an object at night or in the dark. The rod pigment is most sensitive to the light wavelength of $500 \mathrm{~nm}$. Defective or damaged cones result in color deficiency, whereas defective or damaged rods result in problems seeing in the dark and at night.

\subsection{EYE DISEASES}

Diseases that target photoreceptors include age-related macular degeneration and retinitis pigmentosa. The former affects 13 million Americans and is the leading cause of blindness in the western world . Retinitis pigmentosa, while less common, affects 100,000 Americans [2]. Macular degeneration causes loss of the central part of the visual field, making reading impossible. Retinitis pigmentosa initially causes gradual loss of 
peripheral vision followed by loss of central vision, eventually causing complete blindness.

\subsubsection{Age-Related Macular Degeneration}

The macula is a tiny portion of the retina making up only three percent of the whole retina surface [16]. Even though this area is small, it is the most important part of the retina. This is the part of the retina that controls central vision, while the rest of the retina controls peripheral vision. The macula is the highly sensitive area of the retina and is responsible for our critical focusing vision [16]. In ARMD, portions of the retina may become unhealthy, and vision may become affected as the tissues cease to function properly. An image of ARMD affected retina is shown in Fig. 1.5. ARMD is the leading cause of legal blindness in the elderly Caucasian population, but is relatively rare in other races . The degenerative condition of the macula only affects central vision, leaving peripheral vision intact. The primary lesion appears to occur deep within the central retina with deposits known as drusen . Drusen are thought to be metabolic by-products, the increasing deposition of which may further interfere with the high metabolic activity of the macula . There are two basic forms of ARMD dry ARMD and wet ARMD. Both dry and wet ARMD can lead to legal blindness. Dry ARMD is a slow gradual process, while wet ARMD is more rapid form of vision loss. 


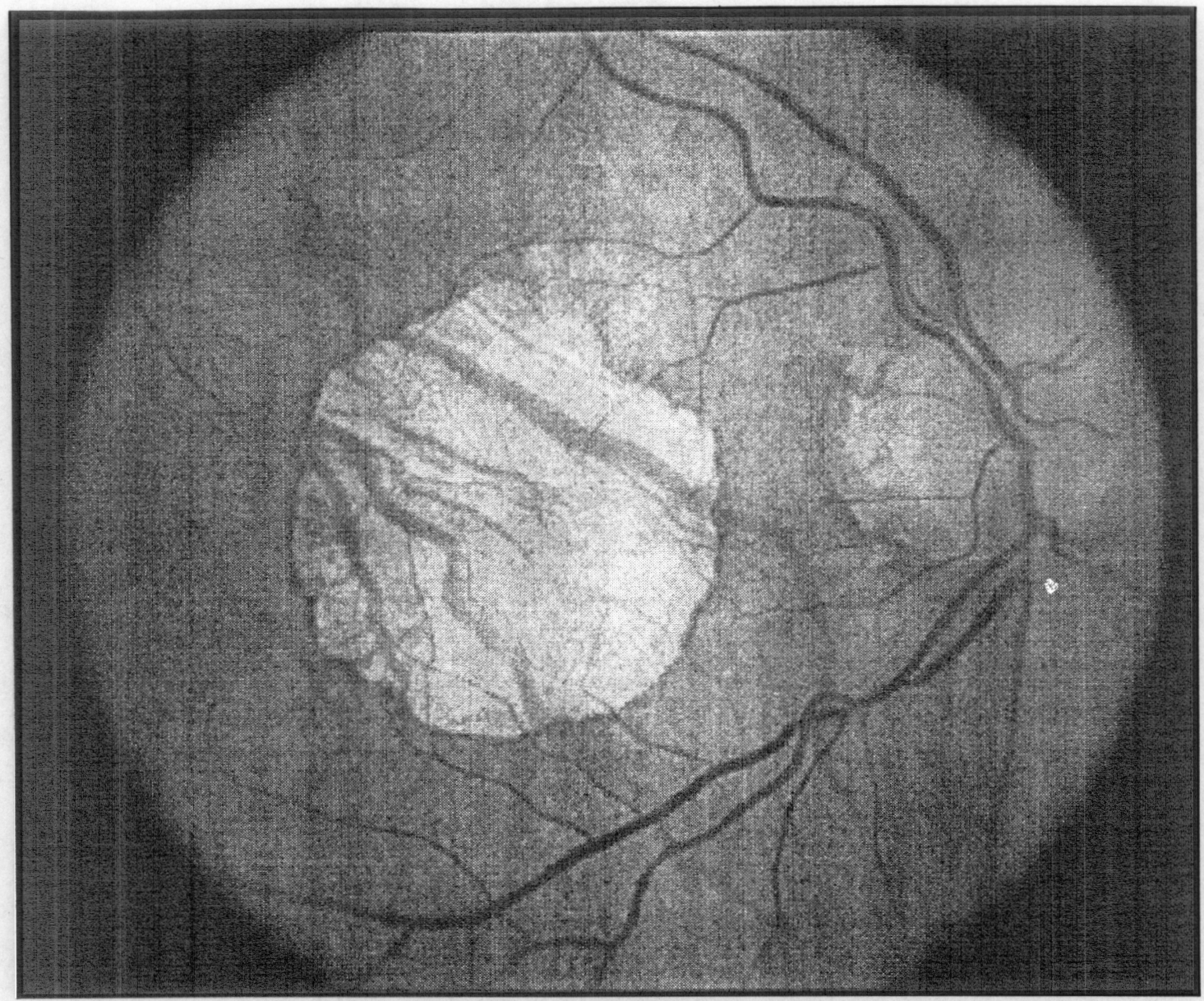

Figure 1.5. Image of ARMD affected retina with permission from Dr. Humayun

\subsubsection{DRY AGE-RELATED MACULAR DEGENERATION}

The deposition of drusen typically begins in the middle-aged and progressively worsens over a period of many years, perhaps several decades [16]. This yellowish deposit is a classic characteristic of Dry ARMD. Drusen results in increasing macular destruction and gradual reduction in central vision. Dry ARMD accounts for $90 \%$ of cases and results in total loss of reading vision over time.

\subsubsection{WET AGE-RELATED MACULAR DEGENERATION}


The wet form of ARMD accounts for only $10 \%$ of all cases form of vision loss than the dry form. This form of the disease occurs when a tiny group of blood vessels breaks through a layer of the retina known as Bruch's membrane, and grows beneath the macula $[4,16]$. This is known as a choroidal neovascular membrane (CNVM). The abnormal vessels of the CNVM may leak fluid causing a localized swelling, or worse, result in localized bleeding. This is the condition rapidly results in legal blindness.

There is no specific treatment for ARMD, though a multitude of studies are in progress in search of prevention or a cure. Treatment for the wet form of ARMD is aimed at minimizing visual loss and/or minimizing the size of the resulting central blind spot. Ophthalmologists may use lasers to destroy the CNVM, or abnormal vessels, in the macula [16], which in turn may also contribute to vision loss.

\subsubsection{Retinitis Pigmentosa}

One of the most devastating conditions affecting the rods is retinitis pigmentosa, an inherited disorder in which the rods gradually degenerate [17]. With time, night vision is severely affected. Eventually, all peripheral vision is destroyed to the point where only central or "tunnel" vision remains $[6,17]$. The disease begins with loss of peripheral vision and night blindness, and often leads to blindness in later life. There is no known treatment; however, since blue and ultraviolet light may aggravate the condition, ambercolored glasses with an ultraviolet absorption coating worn during the day may slow down the progression of the disease. Studies have shown that retinitis pigmentosa is caused by mutations in the rhodopsin gene, the peripherin gene, and possibly in other genes within the rods. 


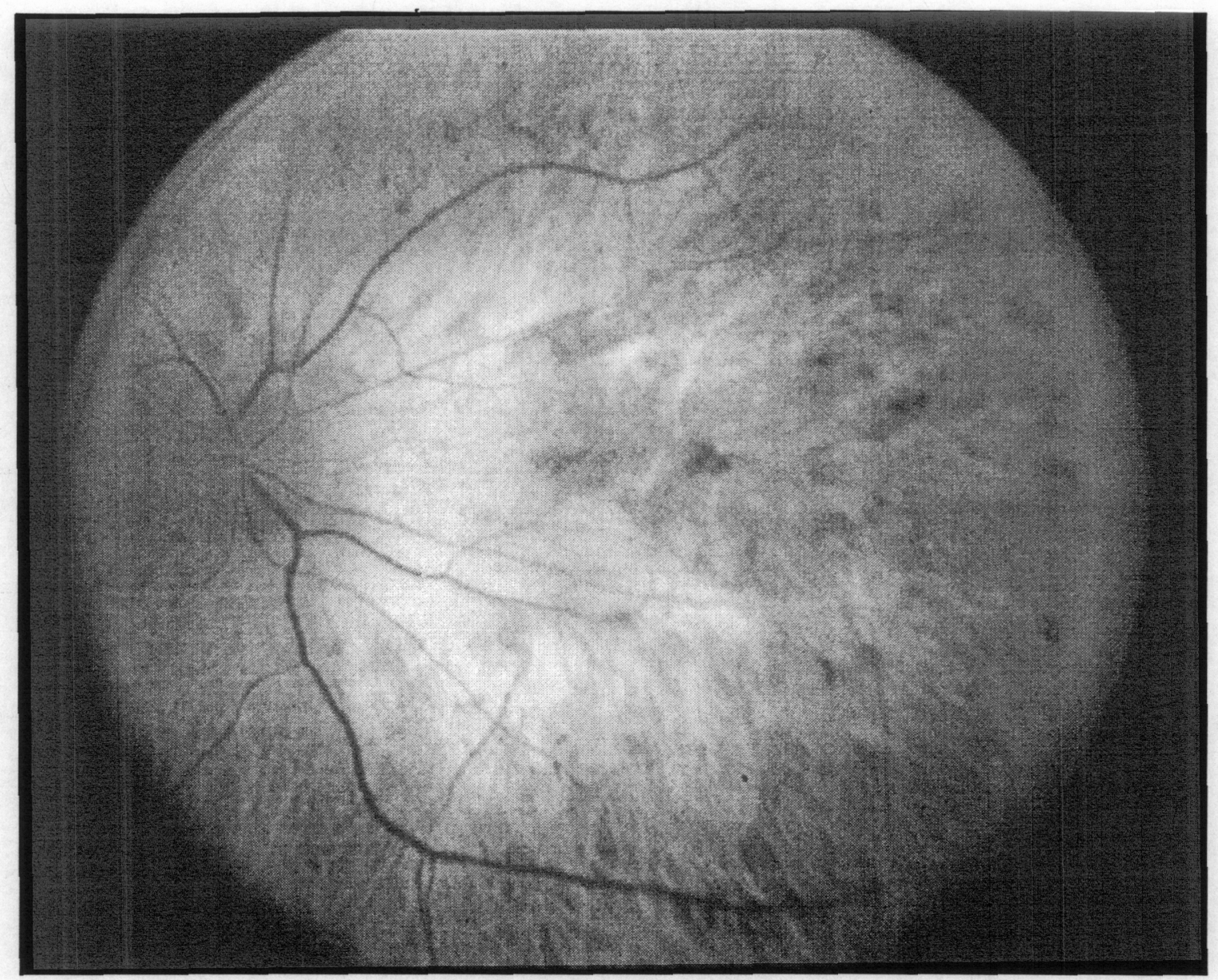

Figure 1.6. The dark spots on the right side of the photo are a classic sign of RP (used with permission from Dr. Mark Humayun)

Most patients present symptoms during the adolescence years with complaints of night blindness and peripheral vision deficits. The retina often shows a classic appearance of pigment deposits in the mid-peripheral retina [17], as shown in Fig. 1.6. Patients also typically have smaller vessels in the retina and paleness of the optic nerves [17].

\subsection{DISCUSSION}


The major causes of blindness are degenerative diseases of the retina. The most common of these are Macular Degeneration and Retinitis Pigmentosa; both targeting photoreceptors. In order to design a suitable retinal implant, one needs to understand the structure and function of the eye and retina. Degeneration of the photoreceptor cells leaves the retina insensitive to light. Therefore, the photoreceptors of the retina recede and the brain no longer receives sufficient information for visual perception. The patient can become blind, despite having a retina with an active connection to the brain through a functioning optic nerve. With RP and ARMD, only the rods and cones in the retina are damaged respectively, sparing the ganglion cells. Knowing the function lost by the eye when diseased, it is possible to develop an implant to substitute for this function. The retinal prosthesis is designed to electrically stimulate the remaining healthy ganglion cells, mimicking the stimulus received from viable photoreceptors. It has been shown that stimulation of this type creates visual images that can be useful to the patient [18]. The retinal implant is a learning, tunable visual prosthesis that bridges and replaces the defect functions of the retina, thereby enabling the group of patients affected by these disorders to regain visual perception.

Several approaches have been made to utilize electronic devices as neuroprostheses, in order to replace the function of damaged sensory neurons. This approach has turned out to be very beneficial in the auditory system [19], where cochlear implants are used to restore hearing to a certain extent. The approach in the visual system is more difficult since the processing information occurs in parallel in many millions of neurons that transform the incoming light stimuli into electrical impulses, process the impulses within the retina in a very complex manner and forward the 
information to the visual cortex [19]. However, hope is spurred from the success of the cochlear implant where 8 to 20 electrodes and sophisticated speech processors have restored hearing to many patients [19]. This success has been greatly attributed to training and the plasticity of the brain.

\subsection{REFERENCES}

[1] L. Hymen, "Epidemiology of eye diseases in the elderly," Eye, vol. 1, pp. 330-41, 1987.

[2] "Statistics on blindness and blinding diseases in the United States," The University of Washington Department of Opthamology, 2002.

[3] J. V. Forrester, A. D. Dick, P. G. McMenamin, and W. R. Lee, The Eye: basic sciences in practice, 2nd Edition ed. London: Harcourt Publishers limited, 2002.

[4] J. Stone, Barlow WE, and M. Humayun, "Morphometric analysis of macular photoreceptors and ganglion cells in retinas with retinitis pigmentosa," Arch. Ophthalmol, vol. 110, pp. 1634-9, 1992.

[5] A. Santos, M. S. Humayun, E. deJuan, R. J. Greenberg, M. J. Marsh, I. B. Klock, and A. H. Milam, "Preservation of the inner retina in retinitis pigmentosa: A morphometric analysis," Archives of Ophthalmology, vol. 115, pp. 511-515, 1997.

[6] M. S. Humayun, M. Prince, E. de Juan, Y. Barron, M. Moskowitz, I. B. Klock, and A. H. Milam, "Morphometric analysis of the extramacular retina from postmortem eyes with retinitis pigmentosa," Investigative Ophthalmology \& Visual Science, vol. 40, pp. 143-148, 1999.

[7] C. W. Oyster, The human eye: structure and function. Sunderland: Sinauer associates, 1999.

[8] M. Erickson, "http://www.stlukeseye.com," 2003.

[9] F. H. Adler, A. Alm, and P. L. Kaufman, Adler's physiology of the eye: Clinical application, 10th ed. St. Louis: Mosby, 2003. 
[10] H. Gray, "Anatomy of the Human Body," vol. 2002: Bartleby.com, 1918.

[11] B. Veisland, "http://wwwVeisland.com," 2003.

[12] D. Pruves, G. J. Augustine, D. Fitzpatrick, K. C. Lawrence, A. S. LaMantia, and J. O. McNamara, "Neuroscience." Sunderland: Sinauer Associates, Inc., 1997.

[13] Retina, "http://www.neurop.ruhr-unibochum.de/CompNsci/BiophysModel/Retina/retina.html," 2003.

[14] S. J. Ryan, "Retina." St. Louis: Mosby, 2001.

[15] W. Krieg, In: Functional Neuroanatomy, 2nd Edition ed. New York: Blakiston, 1953.

[16] C. A. Curcio, N. E. Medeiros, and C. L. Millican, "Photoreceptor loss in age related macular degeneration," Investigative Ophthalmology \& Visual Science, pp. 1236-49, 1996.

[17] E. L. Berson, "Retinitis Pigmentosa," Investigative Ophthalmology \& Visual Science, pp. 1659-76, 1993.

[18] M. Humayun, E. de Juan, G. Dangnelie, R. Greenberg, R. Propst, and P. DH, "Visual perception elicited by electrical stimulation of retina in blind humans," Arch. Ophthalmol, vol. 114, pp. 40-6, 1996.

[19] E. Zrenner, "Will retinal implants restore vision?," Science, vol. 295, pp. 10221025, 2002. 


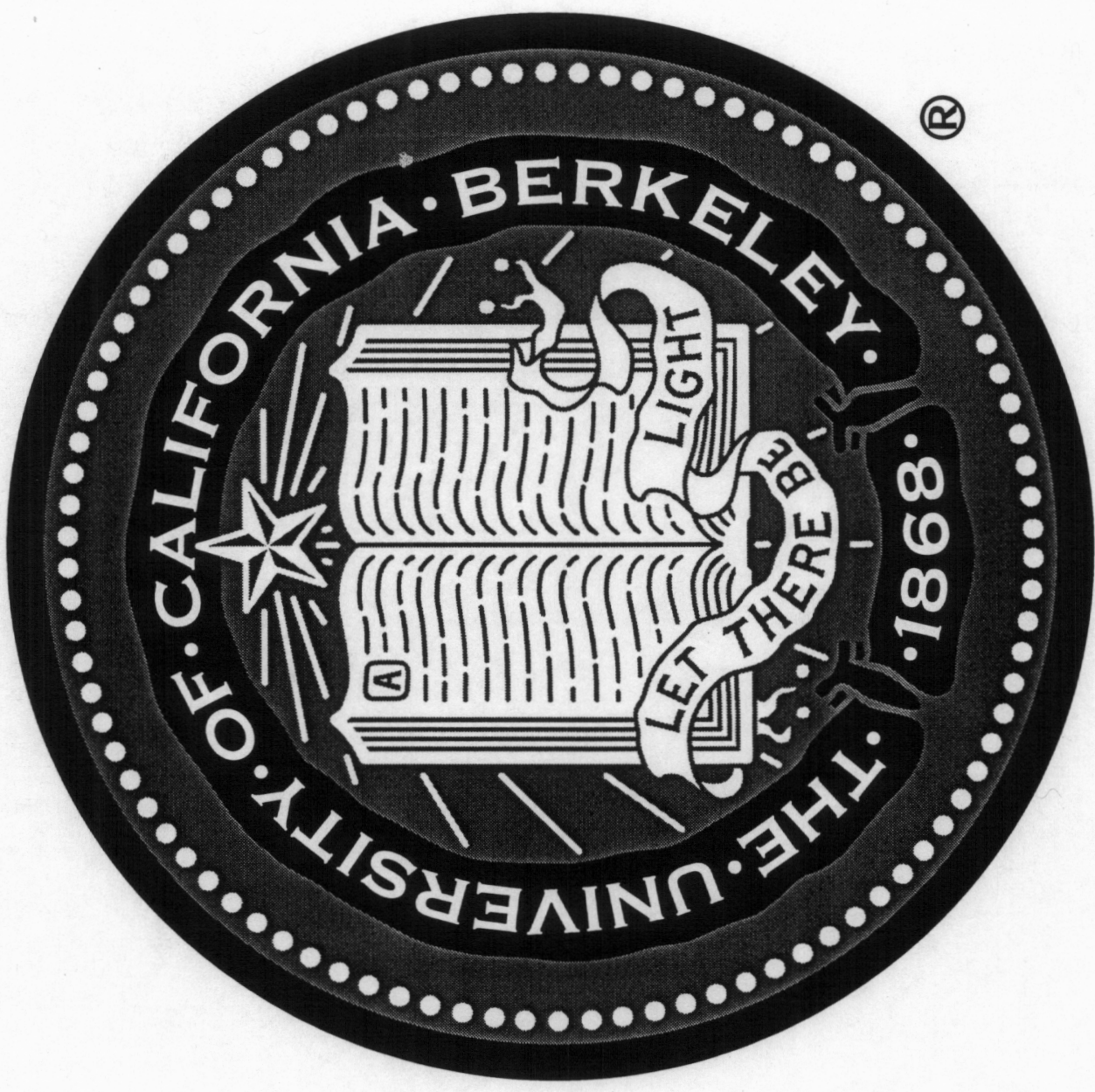




\section{CHAPTER 2: VISUAL PROSTHESES}

\subsection{INTRODUCTION}

This chapter provides an overview of visual prostheses. Out of this modern era of increasing technological ingenuity comes the opportunity for the human brain to design the input it will receive. An example of such a phenomenon is being pursued for the treatment of visual impairments. Research in this area expands the realm of bioengineering, and promises, at the very least, to challenge the boundaries of current microtechnology and further integrate the rapidly expanding fields of electronics and medicine. In 2001, a Department of Energy (DOE) office of Biological and Environmental Research funded a multidisciplinary research consortium, with the explicit goal of creating an epiretinal prosthetic device to overcome the effects of certain types of progressive blindness. The prosthesis is intended to stimulate retinal ganglion cells whose associated photoreceptor cells have been destroyed by age related macular degeneration (ARMD) or retinitis pigmentosa (RP), two currently incurable but widespread conditions $[1,2]$ (see chapter 1 section 1.4$)$. 


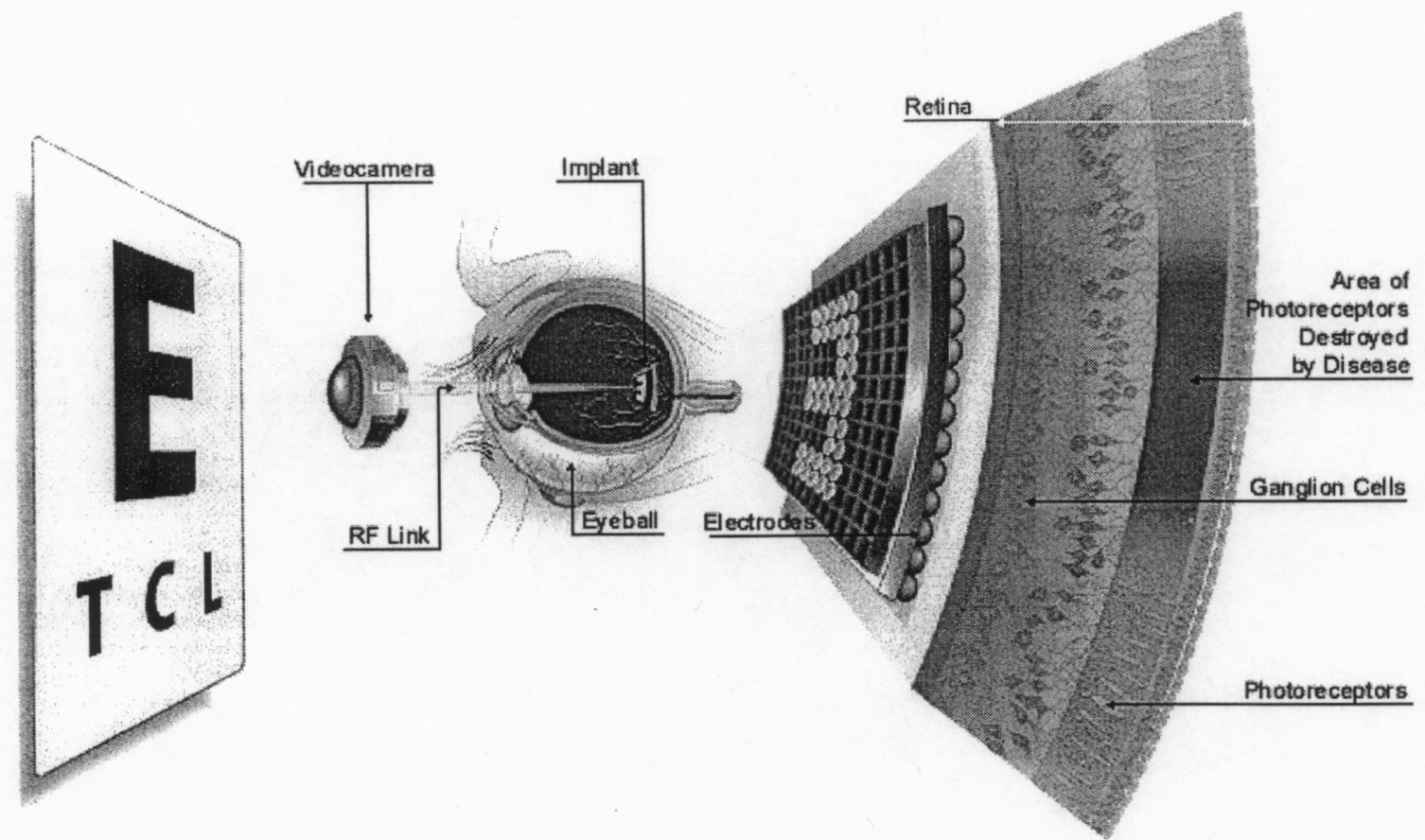

Figure 2.1. Proposed system diagram, with permission from Dr. Humayun, for an epiretinal prosthesis utilizing a video camera and RF link to send image data to a microelectrode array where it is converted into electrical signals to stimulate the retina [3, 4].

The goal of an epiretinal prosthetic device is to bypass degenerate photoreceptors by providing electrical stimulation directly to the ganglion cells [4-7]. Electrical excitation of the ganglion cells is accomplished thru the stimulation a microelectrode array attached onto the surface of the retina as illustrated in Fig. 2.1. This array is driven by an integrated circuit chip (IC) powered by a wireless mechanism such as a laser or RF source mounted on a pair of glasses worn by the patient $[8,9]$ as seen in Fig. 2.1. The IC chip receives real-time visual data input from a small camera, whose output will dictate the spatial and temporal pattern intensity of the signal sent to the implant [7]. The implant will provide an electrical stimulus to the appropriate cells, which will lead to the perception of an image by the brain $[10,11]$. Since the ganglion cells in a healthy retina are stimulated by photoreceptors, a prosthetic visual stimulus will be designed to mimic 
the normal electrical activity within the retinal ganglion cells, in the hope that some measure of sight can be restored to individuals with faulty photoreceptors.

Humayun et al. demonstrated that the majority of the retinal ganglion cells retain function, regardless of the degenerative state of the photoreceptors [5]. By electrically stimulating points on the retina, a visually impaired individual was able to recognize points of light corresponding to those locations activated on the retina [12]. Thus, Humayun's results suggest that replacing the retina with a device capable of translating images into electronic impulses might restore vision. It is these research efforts by Dr. Humayun's team, in collaboration with Second Sight LLC that have instigated the formation of the DOE funded consortium consisting of five national laboratories, two universities, and one private company to develop an epiretinal prosthesis for the visually impaired. This dissertation focuses on the development of a silicone-based microfabricated implantable microelectrode array for the epiretinal prosthesis.

\subsection{VISUAL PROSTHESIS BACKGROUND}

The human body is composed of cells. Our sensory and motor capabilities arise from the properties of various types of nerve and muscle cells, from the complex networks that they form, and from additional supporting cells that maintain a suitable operating environment. Disruption of these cells and networks, caused by either disease or injury, can result in sensory loss or paralysis. Neural prostheses can sometimes compensate for lost function, usually by electrically stimulating viable neurons in pathways where natural connections have been disrupted. The basic idea behind a neuroprosthesis is not new. As early as the late eighteenth century Luigi Galvani and 
Allesandro Volta conducted pioneering experiments, eventually demonstrating that it was possible to stimulate neuronal tissue by means of electricity [13]. Since then, many devices have been developed to supplement neurological functions. Examples are heart pace makers, brain stimulators for Parkinson's disease and epilepsy treatment, bladder control devices, and cochlear implants [14-16]. Among the most successful examples to date is the cochlear prosthesis, which provides auditory sensation to otherwise deaf patients [17]. Deafness often results from loss of the hair cells of the inner ear, which transform the mechanical energy of sound into neural signals that are transmitted to the brain $[14,17]$. Cochlear prostheses electrically stimulate surviving neurons, which are post-synaptic to the hair cells, selectively bypassing the initial parts of the natural auditory pathway [17]. Prolonged use of cochlear implants can lead to dramatic improvements in both speech perception and speech production, and at present there are at least four different types of commercially available devices [17]. Prostheses for the restoration of vision are still at an early stage. However, the first report of a working visual prosthesis based on a cortical implant was reported by Dobelle $e t$ al. in 2002 [18, 19].

A number of approaches, including gene and drug therapies, are currently being pursued with the hope of preventing blindness. Nevertheless, once vision is completely lost, only two of the approaches currently show promise: retinal transplantation [20] and artificial vision via an electronic prosthesis. While introducing this project, it is worthwhile to distinguish the various types of visual prostheses being researched worldwide. It is evident that there are several locations within the human visual pathway which could be electrically stimulated to elicit perception in blind patients, each with 
advantages and disadvantages that will be discussed. For people with severe retinal and optic nerve damage, the only possibility for restoring sight is to directly stimulate the visual cortex through a cortical prosthesis. Another location for a visual prosthesis is the optic nerve. This bundle of cells serves as a transmission line from the eyes to the brain. If an individual has no functional ganglion cells, but has an intact optic nerve, it is possible to evoke the perception of light by stimulating the optic nerve via a cuff electrode [21]. However, if the ganglion cells are functional and only the photoreceptors are damaged then a retinal prosthesis is more suitable. Visual prostheses targeting electrical stimulation of the retina are classified into two types. The subretinal approach stimulates the dorsal side of the retina, whereas the epiretinal approach stimulates the proximal side of the retina. The ultimate objective of any artificial visual aid must be to capture visual data and transmit this information in real-time to the upper-level visual processing center. Although this is unlikely to mimic the detailed percepts obtained by the innate visual system, it should provide enough useful vision for patients to perform such every-day tasks as navigation, recognition, and reading.

\subsection{TYPES OF VISUAL PROSTHESIS}

\subsubsection{Cortical Prosthesis}

Many blind individuals will not benefit from the development of a retinal prosthesis. The reason for this is simple: retinal prostheses rely on the circuitry of the optic nerve to transmit electrical signals from the eye to the center of visual processing in the brain, the visual cortex. If this circuitry is not functional, prostheses must bypass these systems and intervene directly at the cortical level. The group of people for which 
this applies includes anyone who has sustained trauma to the optic nerve, as well as individuals with a severe case of any retinal disease that leaves few functional ganglion cells. Of course, if a cortical visual prosthesis (CVP) works well, a patient that would also qualify for a retinal prosthesis could opt for a CVP instead, although the probability for surgical side effects are much higher [15].

The history of the cortical visual prosthesis began in 1929, when Foerster investigated the effects of electrical stimulation of the occipital lobe of the human cortex. He found that this stimulation caused a subject to see a small point of light, later called a phosphene [22]. This result was reproduced many times after the original experiment with both sighted subjects and blind subjects. The idea that concurrent stimulation of many sites in the brain could produce a single coherent image was postulated as early as 1953 by Krieg [23]. In order for a CVP to be a viable option, a permanent device for chronic stimulation of neural tissue needed to be developed. This was accomplished by Brindley and Lewin [24] in 1967, when the possibility of finding a cure for blindness first became realistic. The neurostimulation device had 80 electrodes, each with its own controlling unit. The 80 telemetrically controlled platinum disk electrodes were successfully implanted above the right visual cortex of a totally blind patient. The set of 80 electrodes lay on top of the occipital cortex [24]. An oscillator with a transmitting coil was tuned to the appropriate frequency to activate a given electrode and pressed against the scalp over the area of the implant [24]. Using this system, Brindley and Lewin were able to demonstrate the plausibility of a permanent CVP.

Brindley and Lewin's patient had suffered retinal damage from glaucoma and was completely blind [24]. Single electrode stimulation of the visual cortex usually produced 
small single spots at a constant position. More importantly, multi-site stimulation had additive effects, resulting in many small points of light being seen simultaneously by the patient. Brindley and Lewin's device was an immediate success. The position of the electrodes corresponded roughly to the position of the phosphenes in the visual field as reported by the patient [24]. One of the major problems researchers reported was that a single electrode could cause many phosphenes to appear. Sometimes this was a function of current, with a greater current leading to the production of more phosphenes, but other times it was independent of stimulus parameters. Additionally, there was a serious limitation on spatial resolution: electrodes spaced closer than $2.4 \mathrm{~mm}$ created a large strip of light when activated simultaneously. The patient also reported seeing phosphene flickering during stimulation. Despite these shortcomings, this work started the investigation into the use of electrical stimulation to overcome vision loss.

The next major advance pertaining to CVPs was made by Dobelle and Mladejovsky in 1974 [25]. They tested various parameters of electrical simulation of human visual cortex on 38 volunteers who were admitted into a hospital for non-elective cranial surgery [25]. Even though they did not implant an actual prosthesis, they were able to provide important data due to the number of patients analyzed compared to Brindley and Lewin's one subject. Some of the results obtained did complement those presented by Brindley and Lewin, yet others directly conflicted. For example, they also found that a single electrode could elicit a multi-phosphene response, and their subjects also reported constant flicker of the phosphenes. However, in contrast to the Brindley and Lewin's work, Dobelle and Mladejovsky concluded that a constant stimulus did not produce a sustained phosphene, but rather one that grew dimmer over time and eventually 
faded after $10-15 \mathrm{sec}$. This is important because it means that an image will have to be refreshed at a particular rate to prevent adaptation. Another important finding was that phosphenes move with eye movements. Finally, the currents required to produce phosphenes were on the order of 3-5 mA [25], a potentially dangerous amount. This added electrical activity was reported to cause seizures [15]. With these problems in mind, researchers continued to work towards an implantable device.

Although these discoveries were milestones in finding a cure for blindness, the less advanced technology of the time prevented any serious progress in the years to follow. It was not until the late 1980's that Brindley and Lewin's important discovery was used as the basis for a number of projects to restore vision. T. Hambrecht of the National Institutes of Health and R. Normann of the University of Utah both head research projects to develop a visual cortical prosthesis. Hambrecht and Normann are two neurobiologists examining the effects of microelectrode stimulation of various regions of the visual cortex [26]. Both of these groups approached the problem of a cortical prosthesis differently than their colleagues in the 1970's. Instead of using surface electrodes on the visual cortex, they chose to employ penetrating microelectrodes. Normann has been working on the development of the Utah Intracortical Electrode Array (UIEA), a 100 microelectrode array designed for recording and stimulating cells in the cortex [27]. These $1 \mathrm{~mm}$ long microelectrodes are fabricated out of silicon with platinum tips and are implanted into the neural tissue. The array is bonded to an electrical stimulation IC and further attached to a planar spiral antenna, which is inductively coupled to an external transmitter coil for power and data telemetry [28]. Thus, the hermetically sealed implant maintains a transcutaneous link with external signal 
processing electronics. Although the long-term safety and stability of the UIEA have not yet been demonstrated, they have proven that the design works and does not immediately damage the neural tissue into which it is inserted [29].

The NIH group describe the various parameters required to produce phosphenes from intracortical microstimulation (ICMS) $[26,30]$. The motivation for this research came from reports of low stimulus thresholds for visual cortex ICMS in primates [26]. Indeed, instead of the 3-5 mA threshold described by Dobelle and Mladejovsky, they reported a more modest range of 20-200 $\mu \mathrm{A}$ depending on depth of insertion, from 3-5 $\mathrm{mm}$ in their human subjects [31]. Upon administration of electrical stimuli to subsurface regions of the visual cortex of a blind patient, the patient identified phosphenes, which varied in color and depth, depending on the location of the stimulus [28]. Moreover, the phosphenes they produced were described as identical to the ones generated by surface stimulation, except that these did not flicker. Also important was the difference in spatial resolution between surface stimulation and ICMS. The NIH group was able evoke the percept of two distinct phosphenes with microelectrodes separated by only $0.7-1.0 \mathrm{~mm}$ [31]. These results; the lower current threshold, absence of flickering, and reduction in phosphene interaction; persuaded investigators to develop higher density intracortical electrodes and abandon surface electrodes.

The NIH CVP team expected to find an optimal set of stimulus parameters, but they found that their subject adapted differently to different aspects of the electrical stimulus, and optimal settings changed over time. For example, the current threshold level required to produce a phosphene increased by an average of $52 \%$ over a sequence of 50 stimuli. During that same interval, the brightness of a given perceived phosphene 
decreased by $75 \%$. These and other issues of accommodation could pose a significant problem in the development of a permanent CVP, because it is difficult to design a device when the specifications are continually varying. Separate from the problem of finding stable stimulus parameters, the NIH group had problems with the actual microelectrode array. By the second month of testing, more than half of the electrodes had broken because they were fabricated out of silicon, a brittle material. Some of this breakage was caused by accidental movement during sleep and some was due to the initial insertion of the array. Obviously, a permanent implant must be viable for much longer than this experimental device.

Another downside to the intracortical approach is that detailed mapping of the phosphene location in the subject's visual field is needed to determine whether the subject could recognize meaningful spatial patterns of stimulation. The reason for this is that the retina's spatial topography corresponds to the cortex's spatial topography only for gross measurements, but not for precise locations. Furthermore, the cortical representation of the visual field is distorted, not linear; stimulation of electrodes in a square grid will not produce the perception of a square. Mapping the location of phosphenes is simple with a sighted subject, but it is significantly more difficult with a blind individual. Without any point of reference, it is difficult for a blind person to reliably correlate the location of phosphenes.

Although the cortical visual prosthesis was conceptualized many decades ago, the technology required to build such a device has recently become available. As the technology advances, devices with more and closer-spaced electrodes will become available, thereby improving resolution. While this raises exciting implications for the 
ability of elucidating the physical arrangement of the neuronal cells involved in the visual pathway, it fails to replicate the experience of sight since the stimuli are independent of external factors. Also, the visual percept is the product of neuronal activity in more than one brain region, a fact that renders rather dubious the proposition that artificial stimulation in any single cortical area could recreate the elaborate perception of vision $[15,28]$. Eventually, the scenario described for retinal prostheses in the introduction will become standard for other visual prostheses; with a video camera processing scenes in real time and transmitting them to a high-resolution microelectrode array. Before any of this can happen, however, studies must be done on the long-term safety of ICMS and the long-term durability of a cortical implant. Furthermore, a useable CVP will require precise eye tracking to stabilize images, an accurate method of mapping phosphenes, and a way to transmit signals to the device without percutaneous connectors in order to minimize the chance of infection.

\subsubsection{Optic Nerve Prosthesis}

Yet another approach to visual prostheses is the electrical stimulation of the optic nerve. The techniques being developed to present visual sensation to blind patients through optic nerve stimulation are currently based on the use of a self-sizing spiral cuff electrode array [21]. In preliminary testing researchers have found that it is highly likely that such a technique may be used in the future to restore limited vision to patients who have non-functioning retinal cells [21]. This technique is performed with a cylindrical cuff electrode array containing disk electrodes along the inside surface, which is placed around the optic nerve near its connection with the eye [15]. Initial tests have been 
performed with a cuff that contains four electrodes, spaced at 90 -degree intervals. The researchers utilized an electrical connection outside the patient's body in order to stimulate the contacts on the cuff in varying positions and combinations. Electrical stimuli applied to the optic nerve produced localized, often colored phosphenes that were reliable up to 118 days after surgery. Changing the pulse duration or frequency resulted in variation of phosphene brightness[21].

The order of axonal fibers within the optic nerve bundle are generally related topographically to the location of their parent ganglion cells on the retinal surface [21], particularly in the region where the nerve exits the eye. Accordingly, fibers near the surface or close to where the nerve connects with ganglion cells near the outer edge of the retina, as well as fibers centrally located within the nerve, attach to ganglion cells closer to the macular region. Thus, because of the analogous topographic mapping of the retinal ganglions to the perceptual space, or visual field of view, excitation of near surface fibers in the optic nerve from the cuff electrode would be expected to elicit percepts in the outer periphery of the visual space. However, encouraging results have been reported wherein patients experience more centrally localized perception closer to the macular region [21], supporting the proposal of optic nerve stimulation as a viable approach to visual prostheses. Perhaps the strongest advantage of this approach is that the challenges of interfacing with the delicate, curved retina surface are circumvented. However, the significant hurdles to be overcome with the optic nerve approach include delivering a sufficiently high level of spatial resolution from the cuff electrode to be practical for a visual prosthesis [21] and achieving preferential stimulation for more centrally located nerve fibers in order to specially target excitation in the fovea region. 


\subsubsection{Retinal Prostheses}

By using electrical stimulation to bypass the non-functional neurons, implanted neural prostheses, such as the cochlear implant, have rehabilitated patients for whom there is no other treatment [14]. It has been proposed that a similar approach could benefit a subset of patients that are blind from ARMD and RP. The results to date have supported this approach and show that vision compatible with mobility and large print reading is possible [11]. Proof of concept work over the years has included: 1) study of the response of retinal ganglion cells to retinal stimulation, 2) development of surgical techniques to implant a device into the eye, 3) methods to stimulate the retina electrically and record the induced responses from the brain, and 4) design and fabrication of prototype implants [15]. Using the knowledge of the cochlear implant, a basic prototype of the prosthesis was used to perform proof of concept experiments with animals, showing that implantable electronics can deliver a visual signal to the brain.

Several groups have focused their efforts on retinal prostheses [8, 32-35]. E. De Juan and M. Humayun demonstrated that the retinal ganglion cells could be electrically stimulated without penetrating the retinal surface $[4,5]$. Patients who were visually impaired due to loss of their rods and cones were capable of sensing a point of light at different positions corresponding to various selected locations where the retina was electrically stimulated by a probe inserted into the ocular cavity. Simultaneous electrical stimulation with two probes placed less than a millimeter apart resulted in two distinct points perceived by the patient. These results suggested that if ganglion cells could be stimulated at an array of locations, a patient might be able to sense distinct shapes. Unlike cortical prostheses, the success of this approach depends on the survival of a 
subpopulation of retinal neurons. Specifically, of critical importance is the survival of the retina's output neurons, the ganglion cells. Degenerative disease such as ARMD and RP fit these criteria. Patients with degenerative diseases have significant populations of healthy ganglion and bipolar cells despite severe photoreceptor loss $[2,36]$. The primary advantages of a retina-based prosthesis are the surgical accessibility of the ganglion cells and the topographic ordering of their receptive fields [37, 38]. Many researchers involved with retinal implant projects hypothesize that higher quality visual perceptions will be experienced with retinal than with optic nerve or intracortical stimulation [39-42]. This proposition is based on the idea that the earlier the electronic input is fed into the nerves along the visual pathway, the clearer the neural signals [42].

There are two current approaches for retinal implants, epiretinal and subretinal as seen in Fig. 2.2. The common goal of the epiretinal and subretinal implants is to restore vision to blind subjects with retinal damage, caused by ARMD and RP. The pioneer groups for the epiretinal prosthesis projects include E. DeJuan and M. Humayun at University of Southern California, J. Wyatt and J. Rizzo of MIT and Harvard, and R. Eckmiller at the University of Bonn in Germany. The epiretinal prosthesis rests on the inside surface of the retina and sends electrical stimulus to the ganglion cells based on information received from an external camera relaying the visual scene. In the subretinal prosthesis approach, a prosthetic device is implanted surgically under the retina, as seen in Fig. 2.2, where the photoreceptors normally lay. The device uses the remaining retinal circuitry to communicate with the brain. A. Chow of Optobionics in Chicago and E. Zrenner of the University of Tubingen, Germany are amongst the leading researchers working on subretinal implants. A diagram of both the epiretinal and subretinal 
prostheses are shown in Fig. 2.2. The use of retinal implants to bypass damaged rods and cones mirrors the successful cochlear implant, which has restored useful hearing to many deaf patients [14].

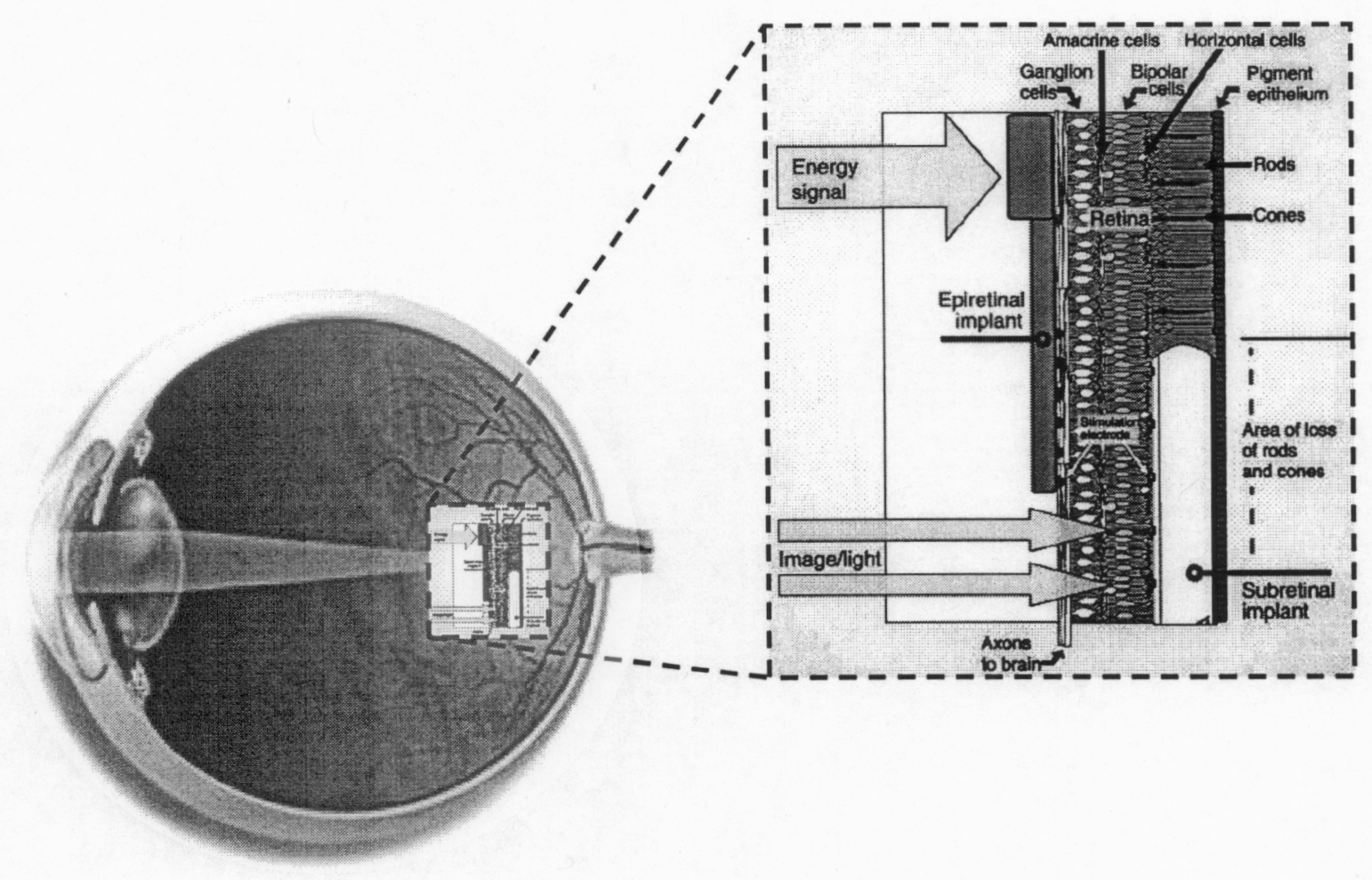

Figure 2.2. Illustration of epiretinal vs.' subretinal prosthesis adapted from [42]

Electrical stimulation studies on animals and humans provide a foundation for the development of retinal prosthesis. With photoreceptor loss due to ARMD and RP, the ganglion cell layer remains largely intact, thereby raising the possibility that an intraocular prosthesis that would stimulate the remaining ganglion cells might provide some vision. Signal transduction of visual input from the photoreceptors requires links to bipolar, horizontal, and amarcine cells [2] (see Chapter 1 section 1.2.3), which 
consequently activate ganglion cells. In turn, the ganglion cells translate the message to visual cortex via the optic nerve. Electrical stimulation of the retinal ganglion layer with an epiretinal implant consisting of an electrode array positioned against the back of the retina may offer a potential pathway for visual input to be processed in a meaningful way by higher-order cortical processes. Researchers have tested this hypothesis by placing bipolar platinum wire electrodes on the surface of the retinas of bullfrog eyecup preparations and rabbit eyes with experimentally induced outer retinal degeneration[43]. Bipolar electrical stimulation of the focal area on the inner retinal surface resulted in an electrically elicited response as evidenced by spike depolarization potentials [43]. This study demonstrates that, despite photoreceptor degradation, stimulation of focal regions of the inner retina can elicit localized sensations of light [44]. The activated areas may in turn accurately depict the position of light and patterns, allowing patients the ability to detect movements and edges [7].

Clinical trials initiated by Humayun et al have been conducted on humans with $A R M D$ and RP to test the viability of the electrical stimulation model for a retinal implant. A discrete percept was elicited in 14 out of 15 patients tested, 13 with their vision impaired by RP and 2 with end-stage ARMD. What is significant about these patients is that almost seventy percent of the ganglion cells retain normal function [45]. Prior to the intraocular procedure, the researchers confirmed ganglion cell and axonal fiber activity by supplying a current through a contact lens electrode[10]. A nonflickering perception was created with stimulating frequencies between 40 and $50 \mathrm{~Hz}$ [46, 47]. The patients were able to identify the stimulated retinal quadrant by the change in position of the visual sensation. This in addition to the demonstration that simultaneous 
electrical stimulation less than $300 \mu \mathrm{m}$ apart resulted in two distinct precepts suggests that multifocal electrical stimulation of the retina might be a viable approach to providing visual input to patients who have profound visual loss due to outer retinal degeneration. Intraocular prostheses have the advantage of delivering input at a proximal point along the afferent visual pathway, but demand a healthy optic nerve to transfer the stimulation to the brain. Thus, patients blind from optic nerve or inner retinal damage, as occurs with glaucoma or diabetes, could not be helped with a retinal prosthesis.

Humayun et al. were able to demonstrate that a $16 \mathrm{~mm}^{2}, 5 \times 5$ array of $400 \mu \mathrm{m}$ platinum electrodes, shown in Fig. 2.3, could be used to stimulate simple images upon the patient's retina. A stimulating current of $600 \mu \mathrm{A}$ passed through a electrode $400 \mu \mathrm{m}$ in diameter elicited a visual percept [4]. Pattern electrical stimulation of the retina was tested in 2 patients. The first patient was able to perceive an activated ' $U$ '-shaped electrode pattern as ' $\mathrm{H}$ '. The second patient correctly identified a square electrode pattern as a 'match box'. Research into the results of electrical stimulation of the retinal surface showed that sensation of light could be produced $[4,34,46,48]$ and that retinal neurons are preserved after the death of photoreceptors in ARMD and RP $[2,5,44,49-53]$. It has been shown by Dr. Humayun's group that an implant can be placed on the retinal surface with no significant damage to the retinal architecture beneath the array and no significant decline in visual function $[4,10]$. Building upon these medical results, research teams at University of Southern California, Massachusetts Institute of Technology, Fraunhofer Institute and other institutions have worked towards developing prototypes for a retinal prosthesis. The different teams have their own way of solving this challenging task of simulating vision, yet the basic concept is much the same. 


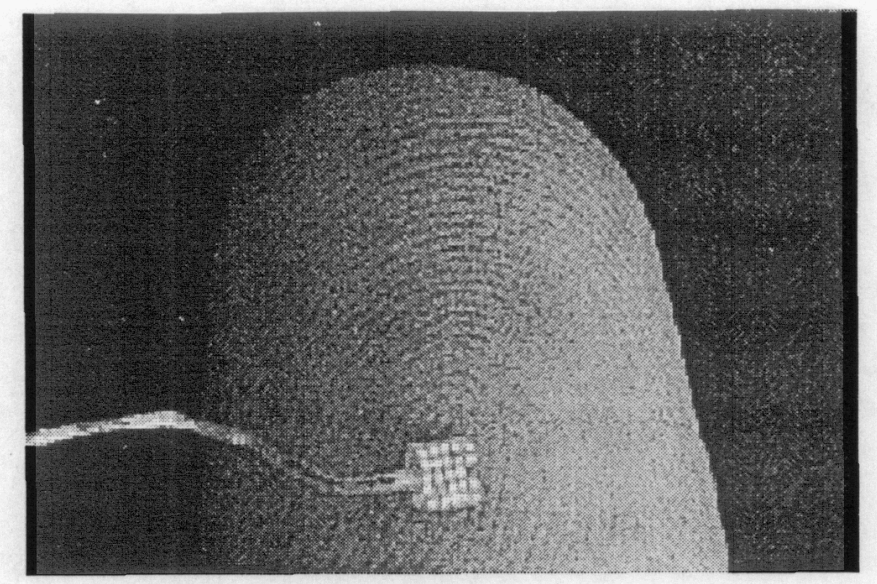

Figure 2.3. Silicone encapsulated array of 25 electrodes used to produce shapes on the retina of patients with permission from Dr. Humayun [4].

\subsubsection{SUBRETINAL PROSTHESIS}

Several research efforts are pursuing the subretinal approach $[54,55]$, which is based on replacing an area of destroyed photoreceptor with an implanted microphotodiode array (MPDA) [55, 56]. This MPDA consists of 2000-5000 densely packed electrodes on 3mm diameter disk fabricated out of silicon [33]. The MPDA is inserted between the outer retina and the retinal pigment epithelium, in place of the defective photoreceptor layer. This physiological position will allow for maximum utilization of the signal-processing network that is responsible for compressing the information from more than 100 million photoreceptors to 1 million ganglion cells [42, 54, 57]. Incoming light passes through the inner layers of the retina, but instead of reaching normal photoreceptor cells, light hits the MPDA [55]. At this point, the MPDA would be stimulated to create electrical impulses that emulate the response of normal photoreceptors. These impulses in turn stimulate retinal nerve cells in order to contribute to the overall signal that is propagated through the optic nerve. One of the difficulties 
faced in perfecting the subretinal MPDA method is the actual interaction of the photodiodes with the neurons that normally receive information from photoreceptors. An attractive aspect of the subretinal approach is that its integrated circuits faces towards the pupil, making it possible to derive its power from the incident light entering the eye. The main challenge is the maximization of the signal picked up by the neurons. There is only a very tiny charge produced by a single photodiode when fueled by outside light, not enough to stimulate the neurons. To overcome this issue an external energy source is needed. In this case incident light hitting the MPDA will serve as a switch to the external energy source to deliver current to the contacting retinal neurons.

Furthermore, a hermetically sealed device positioned posterior to the retina with no communication mechanism with the external world does not facilitate configuration or programming of stimulation pulse parameters, such as amplitudes, pulse widths, interphase delay and stimulation frequency which have been found to influence the quality of perception $[40,56]$. Another potential disadvantage of the subretinal approach is that inner retinal placement of the implant may lead to retinal damage through retinal separation or obstruction of blood flow from the choroids into the retina [58]. The cells in the area surrounding the implant may be blocked off from necessary life-sustaining nutrients [58], because the current design consists of a tightly packed diode array on a solid, piece of titanium, plastic, or silicon $[33,54]$.

By electrically stimulating the retina from the posterior, as in the subretinal system, the signal flow through this network may be preserved in a manner consistent with natural biological vision. This approach is appealing due to its design to maximize the utilization of the already existing signal-processing network while minimizing the 
technical components needed for its operation. However, as stated earlier a postprocessing and power amplification of the photodiode output will probably be necessary for efficient stimulation with the subretinal approach [59]. In contrast, the epiretinal prosthesis is equipped with current sources that will be optimized with respect to stimulation efficiency.

\subsubsection{EPIRETINAL PROSTHESIS}

Several research groups have adopted the epiretinal approach [32, 34, 60-63], which in contrast to the subretinal arrangement, seeks to stimulate the ganglion cells directly from the inner surface of the retina, as shown in Fig. 2.2. The epiretinal prosthesis will consist of an external miniature low-power camera, worn in an eyeglass frame that will capture an image and transfer the visual information plus power to the intraocular components. The intraocular prosthesis will decode the signal and electrically stimulate the retinal neurons through the electrodes in a manner that corresponds to the image acquired by the camera. A generally common characteristic among the epiretinal development efforts is partitioning of the system into an intraocular unit, consisting of the retinal stimulator, and an extraocular unit providing front end signal processing. Because the epiretinal device does not rely on the natural data processing of the neural compartments in the retina, all signal encoding is synthesized. Hence, the epiretinal approach requires an encoder for mapping visual patterns into pulse trains as inputs for electronic stimulation. An advantage to having an external signal-processing unit is it facilitates refinement or upgrading of the device. This partitioning becomes necessary when the surgical placement and orientation of the implant exacerbates the delivery of 
power and video input, especially when the active side of the integrated circuit faces inwards towards the retinal surface. The two units are typically coupled with a wireless power and data telemetry mechanism, which can take several forms. Some researchers are studying optical power telemetry based on a laser diode [64], while others are considering inductively coupled power and data telemetry $[9,63]$. The strengths of the epiretinal approach derive from delegating most or all of the image acquisition and processing algorithms to an extraocular position[8]. Excluding all components except for the receiver coil, power/data recovery, and the retinal stimulator, results in smaller, lower-power intraocular unit, while also preserving the flexibility of upgrading the image processing hardware without performing additional surgery. However, by stimulating the ganglion cells from the vitreo retinal side, the natural image processing in the retina's neural network is now largely bypassed, unlike the subretinal approach [55]. Accordingly, the extraocular unit may now be required to compensate for the bypassed neural network so retinal simulation is delivered in a way that is more similar to natural synaptic input [11]. Efforts are underway to integrate this retinal functionality into the epiretinal design by emulating the ganglion cell receptive field in an extraocular signal processor $[34,65]$.

Another potential disadvantage of the epiretinal approach relates to mechanical anchoring of the implant and long-term stability of the interface at the inner retinal surface. Unlike the subretinal approach, where the implant may receive some mechanical support from the tissues posterior to the retina [66], an epiretinal placement of the stimulator at the vitreo retinal surface imparts the weight and inertial forces of the implant onto the fragile retina $[26,32]$. The retina is known to be very sensitive and 
reactive to physical stresses. It is susceptible to tears, bleeding, and detachment or to cellular overgrowth following surgery; potentially compromising the rental electrode interface $[32,64]$. A proposed solution adopted by those favoring the epiretinal approach is to anchor the integrated circuit portion of the implant, including the stimulator IC, at a more mechanically stable position, such as the vitreous base [32], in the crystalline lens cavity or the midviterous [67]. From here, the stimulator IC is attached to a thin conformable electrode array with embedded interconnect to route stimulation currents from the stimulator IC to the vitreo retinal surface. The end attached to the retina provides platinum or iridium oxide surface electrodes[15] and is anchored to the retina with titanium tacks or biological adhesives $[68,69]$. This reduces stress on the retina while also relocating the implant electronics away from the retinal surface to the anterior chamber, thus removing IC generated heat from direct contact with the retina and also improving inductive telemetry through reducing the coupling distance. However, this raises the challenge of manufacturing a thin conformable electrode array in a narrow width in order to be surgically implantable, while remaining mechanically robust. A further complication is introduced as the interconnect must feed through the sealed implant encapsulate to attach to the stimulator electrodes without compromising the functionality of the implant. Both of these issues will be addressed in this dissertation.

Both the subretinal and epiretinal approaches benefit from the close association existing between the placement of the retinal stimulation and the localized perception within a patient's visual field. This is a direct consequence of the topography of ganglion cells [37] and remaining neuron cell types across the retina. Since a wide field of view and peripheral vision relate to the large coverage of the retinal within the posterior half of 
the eye, the stimulation of a small retinal area is expected to elicit a restricted field of view or tunnel vision. Research in flexible structures may yield electrode arrays that can adapt to the retinal curvature with the possibility of stimulating a greater retinal area. However, because both current subretinal and epiretinal approaches target a small area of the retina near the fovea, limiting the field of view. Yet, beyond these observations there is no conclusive evidence whether the subretinal or epiretinal approach will yield a more effective chronic retinal prosthesis. Some believe that the final retinal prosthesis will encompass the best technological advancements from both the subretinal and epiretinal research efforts.

\subsection{DISCUSSION}

During the past 12 years, scientists have gradually tackled the many different components necessary to create a working implant and have had success in most areas. Basic prototypes, with similar designs, have been created by each of the major teams $[15$, $54,59,70]$. The teams have also performed acute and chronic experiments with animals proving that implantable electronics can deliver a visual signal to the brain. Even more recently teams have begun long-term experiments on blind human test subjects. Subjects reported seeing different shades of light and some outlines of objects $[11,54]$.

Dr. Humayun's team at the University of Southern California's (USC) Keck School of Medicine implanted, chronically, two retinal prostheses in 2 blind human volunteers as part of an FDA-approved feasibility trial in 2002 [11]. Second Sight LLC developed these 16 electrode microelectronic retinal implants. The total area of the device is $20 \mathrm{~mm}^{2}$ [11] as seen in Fig. 2.4. Previously, components of a retinal prosthesis 
have been tested acutely, always removed within an hour of implantation, in human patients. Under current testing protocol, while chronic prosthesis will be permanent barring serious complications, the device is switched on only under medical supervision so the quality of perception that is attained with electrical stimulation of the retina is closely monitored. The results so far have been promising with patients demonstrating the ability to distinguish light from dark and large letters $[6,11,47]$. These findings are very promising; scientists are coming closer to creating a permanent implant to restore vision to patients suffering from retinal damage.

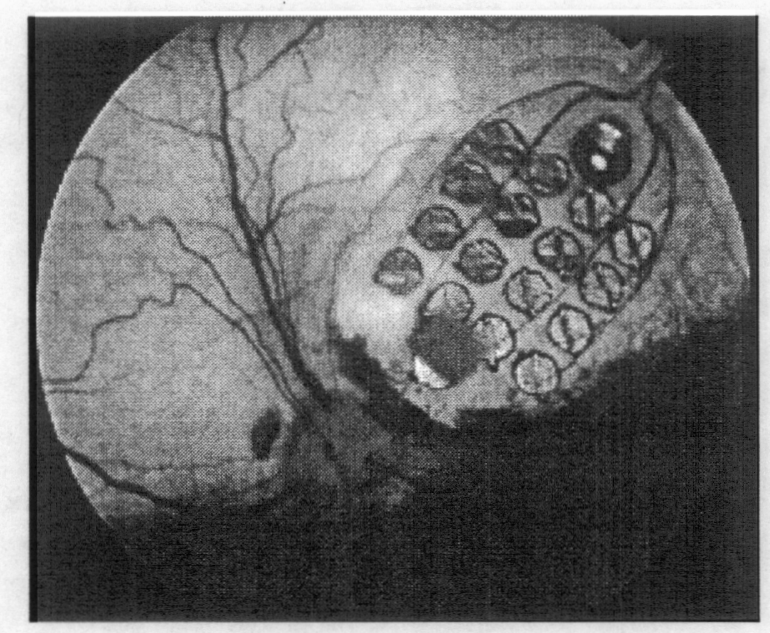

Figure 2.4. $4 \mathrm{X} 4$ electrode array implants developed by Second Sight LLC. Image used with permission from Drs. Humayun and Greenberg

Microelectronic retinal, cortical, and optic nerve implants are current research underway to restore useful sight to those with severe vision loss by replacing a defect or missing link along the visual pathway. Retinal and optic nerve implants require an intact visual pathway to the brain to allow them to function. While cortical implants can serve the needs of most blind patients regardless of where the damage is along the visual pathway and as long as the brain is functioning properly. However, unnecessarily opting 
to bypass functioning visual pathway route risks minimizing the capability of obtaining a more "natural" visual percept.

The visual prosthesis field is less mature than those surrounding other implants. So far researchers have made tremendous progress on the path to producing a viable implant to restore vision to some blind patients. Also, the test results on the prototype implants show that human patients have only been able to see lights, or outlines of objects. In the future the goal of this visual prostheses is to develop an implant that is capable of allowing patients to see complex or even perfect images. The present state of visual prosthetics does not seem to have improved dramatically in proportion to the time since Brindley's work. However, with advances in computer technology, miniaturization of electrodes, and creation of improved microchips the prospect exists that future devices might be developed that will enable millions of blind patients to obtain useful range of vision. There is no question about the benefits or the phenomenal impact on our society that an implantable retina used to alleviate some forms of blindness would have. So far the experiments performed to demonstrate the feasibility of using a prosthesis to restore vision have all been promising. However there is still considerable scientific research and technology development that needs to be undertaken in order to produce a finished, working, and biocompatible implant. Presently, retinal implants are at an early stage of development. 


\subsection{REFERENCES}

[1] M. S. Humayun, M. Prince, E. de Juan, Y. Barron, M. Moskowitz, I. B. Klock, and A. H. Milam, "Morphometric analysis of the extramacular retina from postmortem eyes with retinitis pigmentosa," Investigative Ophthalmology \& Visual Science, vol. 40, pp. 143-148, 1999.

[2] J. Stone, Barlow WE, and M. Humayun, "Morphometric analysis of macular photoreceptors and ganglion cells in retinas with retinitis pigmentosa," Arch. Ophthalmol, vol. 110, pp. 1634-9, 1992.

[3] DOE, "A High-Density Microelectronic-Tissue Hybrid Sensor for Imaging," DOE LAB 01-14 Biomedical Engineering Program, 2001.

[4] M. Humayun, E. de Juan, G. Dangnelie, R. Greenberg, R. Propst, and P. DH, "Visual perception elicited by electrical stimulaiton of retina in blind humans," Arch. Ophthalmol, vol. 114, pp. 40-6, 1996.

[5] M. Humayun, "Is surface electrical stimulation of the retina a feasible approach towards the development of a visual prosthesis?," in Biomedical Engineering. Chapel Hill: University of North Carolina, 1994.

[6] M. S. Humayun, E. De Juan, G. Dagnelie, R. J. Greenberg, R. H. Prost, and D. H. Phillips, "Visual Perception Elicited by Electrical Stimulation of Retina in Blind Humans," Archives of Ophthalmology, vol. 114, pp. 40-46, 1996.

[7] J. Weiland, M. Humayun, and G. Dangnelie, "Understanding the origin of visual percepts elicited by electrical stimulation of the human retina," Arch. Clin Exp Ophthalmol, vol. 237, pp. 1007-13, 1999.

[8] M. Humayun and E. de Juan, "Artificial vision," Eye, vol. 12, pp. 605-7, 1998.

[9] M. Clements, K. Vichienchom, W. T. Liu, C. Hughes, E. McGucken, C. DeMarco, J. Mueller, M. Humayun, E. de Juan, J. D. Weiland, and R. Greenberg, "An implantable neuro-stimulator device for a retinal prosthesis," International Solid-state circuits conference, pp. 216-17, 1999. 
[10] M. Humayun, E. de Juan, J. Weiland, G. Dangnelie, S. Katona, R. Greenberg, and S. Suzuki, "Pattern electrical stimulation of the human retina," Vision research, vol. 39, pp. 2569-76, 1999.

[11] E. Margalit, M. Maia, J. D. Weiland, R. J. Greenberg, G. Y. Fujii, G. Torres, D. V. Piyathaisere, T. M. O'Hearn, W. T. Liu, G. Lazzi, G. Dagnelie, D. A. Scribner, E. de Juan, and M. S. Humayun, "Retinal prosthesis for the blind," Survey of Ophthalmology, vol. 47, pp. 335-356, 2002.

[12] W. T. Liu, K. Vichienchom, M. Clements, C. DeMarco, C. Hughes, E. McGucken, M. Humayun, E. de Juan, J. D. Weiland, and R. Greenberg, "A neurostimulus chip with telemetry unit for retinal prosthetic device," IEEE Journal of Solid-State Circuits, vol. 35, pp. 1487-97, 2000.

[13] G. S. Brindley, "The site of electrical excitation of the human eye," J. Physiol., vol. 127, pp. 189-200, 1955.

[14] J. P. Rauschecker and R. V. Shannon, "Sending sound to the brain," Science, vol. 295, pp. 1025-29, 2002.

[15] M. Humayun, "Intraocular Retinal Prosthesis," Tr. Am. Ophth. Soc, vol. 99, pp. 271-300, 2001.

[16] P. Heiduschka and S. Thanos, "Implantalbe Bioelectronic interfaces for lost nerve functions," Progress in Neurobiology, vol. 55, pp. 433-461, 1998.

[17] P. C. Loizou, "Mimicking the human ear," IEEE Signal Processing Magazine, vol. 15, pp. 101-103, 1998.

[18] W. H. Dobelle, "Artificial vision for the blind by connecting a television camera to the visual cortex," ASAIO Journal, vol. 46, pp. 3-9, 2000.

[19] W. H. Dobelle, "The Dobelle group at the Dobelle Institute, Inc," vol. October $2002,2002$.

[20] M. S. Humayun, E. de Juan, M. del Cerro, G. Dagnelie, W. Radner, S. R. Sadda, and C. del Cerro, "Human neural retinal transplantation," Investigative Ophthalmology \& Visual Science, vol. 41, pp. 3100-3106, 2000. 
[21] C. Veraart, C. Raftopoulos, and J. Mortimer, "Visual sensation produced by optic nerve stimulation using an implanted self-sizing spiral cuff electrode," Brain Research, vol. 813, pp. 181-186, 1998.

[22] O. Foerster, "Beitrage zur pathophysiologie der sehbahn und der sehephare," $J$. Psychol. Neurol. Lpz, vol. 39, pp. 463-485, 1929.

[23] W. Krieg, In: Functional Neuroanatomy, 2nd Edition ed. New York: Blakiston, 1953.

[24] G. S. Brindley and W. S. Lewin, "The sensations produced by electrical stimulation of the visual cortex," J. Physiol., vol. 196, pp. 479-493, 1968.

[25] W. H. Dobelle and W. G. Mladejovsky, "Phosphenes produced by electrical stimulation of human occipital cortex and their application to the development of a prosthesis for the blind," J. Physiol., vol. 243, pp. 553-576, 1974.

[26] R. A. Normann, E. M. Maynard, K. S. Guillory, and D. J. Warren, "Cortical Implants for the Blind," IEEE Spectrum, vol. 33, pp. 54+, 1996.

[27] E. M. Maynard, C. T. Nordhausen, and R. Normann, "The Utah Intracortical Electrode Array: a recording structure for potential brain-computer interfaces," Electroencephalogr. Clin. Neurophysiol., vol. 102, pp. 228-239, 1997.

[28] R. A. Normann, E. M. Maynard, P. J. Rousche, and D. J. Warren, "A neural interface for a cortical vision prosthesis," Vision Research, vol. 39, pp. 2577 $2587,1999$.

[29] P. J. Rousche and R. Normann, "Chronic intracortical microstimulation (ICMS) of cat sensory cortex using the Utah Intracortical Electrode Array," IEEE Trans. Rehab. Eng., vol. 7, pp. 56-68, 1999.

[30] M. Bak, J. P. Girvin, F. T. Hambrecht, C. V. Kufta, G. E. Loeb, and E. M. Schmidt, "Visual sensations produced by intracortical microstimulation of the human occipital cortex," Med.\&Biol. Eng. \&Comput., vol. 28, 1990.

[31] E. M. Schmidt, M. Bak, F. T. Hambrecht, C. V. Kufta, D. K. O'Rourke, and P. Vallabhanath, "Feasibility of a visual prosthesis for the blind based on intracortical microstimulation of the visual cortex," Brain, vol. 119, 1996. 
[32] J. Rizzo and J. Wyatt, "Prospects for a visual prosthesis," Neuroscientiest, vol. 3, pp. 251-62, 1997.

[33] E. Zrenner, K. D. Miliczek, V. P. Gabel, H. G. Graf, E. Guenther, H. Haemmerle, B. Hoefflinger, K. Kohler, W. Nisch, M. Schubert, A. Stett, and S. Weiss, "The development of subretinal microphotodiodes for replacement of degenerated photoreceptors," Ophthalmic Research, vol. 29, pp. 269-280, 1997.

[34] R. Eckmiller, "Learning retina implants with epiretinal contacts," Ophthalmic Research, vol. 29, pp. 281-289, 1997.

[35] A. Chow and V. Chow, "Subretinal electrical stimulation of the rabbit retina," Neurosci Lett, vol. 225, pp. 13-6, 1997.

[36] A. Santos, M. S. Humayun, E. deJuan, R. J. Greenberg, M. J. Marsh, I. B. Klock, and A. H. Milam, "Preservation of the inner retina in retinitis pigmentosa: A morphometric analysis," Archives of Ophthalmology, vol. 115, pp. 511-515, 1997.

[37] C. A. Curcio and K. A. Allen, "Topography of ganglion cells in the human retina," The Journal of Comparative Neurology, vol. 300, pp. 5-25, 1990.

[38] J. F. Rizzo, M. M. Socha, D. Edell, B. Antkowiak, and D. Brock, "Development of a silicone retinal implant: surgical methods and mechanical design," Investigative Ophthalmology \& Visual Science, vol. 35, pp. 1380, 1994.

[39] J. Ohta, N. Yoshida, K. Kagawa, and M. Nunoshita, "Proposal of application of pulsed vision chip for retinal prosthesis," Japanese Journal of Applied Physics Part 1-Regular Papers Short Notes \& Review Papers, vol. 41, pp. 2322-2325, 2002.

[40] N. S. Peachey and A. Y. Chow, "Subretinal implantation of semiconductor-based photodiodes: Progress and challenges," Journal of Rehabilitation Research and Development, vol. 36, pp. 371-376, 1999.

[41] J. F. Rizzo, J. Wyatt, M. Humayun, E. de Juan, W. T. Liu, A. Chow, R. Eckmiller, E. Zrenner, T. Yagi, and G. Abrams, "Retinal prosthesis - An encouraging first decade with major challenges ahead," Ophthalmology, vol. 108, pp. 13-14, 2001. 
[42] E. Zrenner, "Will retinal implants restore vision?," Science, vol. 295, pp. 1022$1025,2002$.

[43] M. Humayun, R. Propst, E. Dejuan, K. McCormick, and D. Hickingbotham, "Bipolar Surface Electrical Stimulation of the Vertebrate Retina," Archives of Ophthalmology, vol. 112, pp. 110-116, 1994.

[44] A. Majji, M. Humayun, and J. Weiland, "Long-term histological and electrophysiological results of an inactive epiretinal electrode array implantation in dogs," Invest Ophthalmol Bis Sci, vol. 40, pp. 2073-81, 1999.

[45] W. Roush, "Ophthalmology - Envisioning an Artificial Retina," Science, vol. 268, pp. 637-638, 1995.

[46] M. S. Humayun, E. de Juan, J. D. Weiland, G. Dagnelie, S. Katona, R. Greenberg, and S. Suzuki, "Pattern electrical stimulation of the human retina," Vision Research, vol. 39, pp. 2569-2576, 1999.

[47] J. D. Weiland, M. S. Humayun, G. Dagnelie, E. de Juan, R. J. Greenberg, and N. T. Iliff, "Understanding the origin of visual percepts elicited by electrical stimulation of the human retina," Graefes Archive for Clinical and Experimental Ophthalmology, vol. 237, pp. 1007-1013, 1999.

[48] M. Humayun, E. de Juan, and J. Weiland, "Pattern electrical stimulation of the human retina," Vision research, vol. 39, pp. 2569-76, 1999.

[49] M. S. Eckmiller, "Distal Invaginations and the Renewal of Cone Outer Segments in Anuran and Monkey Retinas," Cell and Tissue Research, vol. 260, pp. 19-28, 1990.

[50] M. S. Eckmiller, "Morphogenesis and renewal of cone outer segments," Progress in Retinal and Eye Research, vol. 16, pp. 401-441, 1997.

[51] M. Humayun, R. Propst, E. de Juan, K. McCormick, and D. Hichingbotham, "Bipolar surface electrical stimulation of the vertebrate retina," Arch. Ophthalmol, vol. 112, pp. 110-16, 1994.

[52] A. Y. Chow and V. Y. Chow, "Subretinal electrical stimulation of the rabbit retina," Neuroscience Letters, vol. 225, pp. 13-16, 1997. 
[53] K. Kohler, E. Zrenner, and R. Weiler, "Ethambutol Alters Spinule-Type Synaptic Connections and Induces Morphologic Alterations in the Cone Pedicles of the Fish Retina," Investigative Ophthalmology \& Visual Science, vol. 36, pp. 1046$1055,1995$.

[54] A. Y. Chow, M. T. Pardue, J. I. Perlman, S. L. Ball, V. Y. Chow, J. R. Hetling, G. A. Peyman, C. P. Liang, E. B. Stubbs, and N. S. Peachey, "Subretinal implantation of semiconductor-based photodiodes: Durability of novel implant designs," Journal of Rehabilitation Research and Development, vol. 39, pp. 313$321,2002$.

[55] E. Zrenner, V. P. Gabel, H. Haemmerle, B. Hoefflinger, and M. Schubert, "Subretinal implants," Ophthalmic Research, vol. 30, pp. 197-198, 1998.

[56] A. Y. Chow and N. S. Peachey, "The subretinal microphotodiode array retinal prosthesis," Ophthalmic Research, vol. 30, pp. 195-196, 1998.

[57] E. Zrenner, "The subretinal implant: Can microphotodiode arrays replace degenerated retinal photoreceptors to restore vision?," Ophthalmologica, vol. 216, pp. 8-20, 2002.

[58] K. Kohler, J. A. Hartmann, D. Werts, and E. Zrenner, "Retinal degeneration and biocompatibility of subretinal implants: a histological analysis," Ophthalmologe, vol. 98, pp. 364-368, 2001.

[59] E. Zrenner, F. Gekeler, V. P. Gabel, H. G. Graf, M. Graf, E. Guenther, H. Haemmerle, B. Hoefflinger, K. Kobuch, K. Kohler, W. Nisch, H. Sachs, B. Schlosshauer, M. Schubert, H. Schwahn, M. Stelzle, A. Stett, B. Troeger, and S. Weiss, "Subretinal microphotodiode arrays to replace degenerated photoreceptors?," Ophthalmologe, vol. 98, pp. 357-363, 2001.

[60] J. Wyatt and J. F. Rizzo, "Ocular Implants for the Blind.," EEE Spec., vol. I 33, pp. 47-53, 1996.

[61] T. Yagi, Y. Ito, H. Kanda, S. Tanaka, M. Watanabe, and Y. Uchikawa, "Hybrid retinal implant: fusion of engineering and neuroscience," presented at IEEE International conference on systems, man, and cybernetics, 1999.

[62] T. Yagi, Y. Ito, M. Watanabe, T. Matsushima, and Y. Uchikawa, "A study on hybrid artificial retina with cultured neural cells and semi-conductor 
microdevice," presented at IEEE International Joint conference on Neural networks, 1998.

[63] G. J. Suaning, N. H. Lovell, K. Schindhelm, and M. T. Coroneo, "The bionic eye(electronic visual prosthesis): A review," Aust. NZ J. Oph-thal., vol. 26, pp. 195-202, 1998.

[64] J. Wyatt, J. Mann, D. Edell, J. Raffel, and J. F. Rizzo, "Development of a silicon retinal implant: demonstration of microelectronic system for optical transmission of signal and power," Investigative Ophthalmology \& Visual Science, vol. 36, pp. $1130,1995$.

[65] R. Eckmiller, R. Hunermann, and M. Becker, "Exploration of a dialog-based tunable retina encoder for retina implants," Neurocomputing, vol. 26-7, pp. 1005$1011,1999$.

[66] M. T. Pardue, E. B. Stubbs, J. I. Perlman, K. Narfstrom, A. Y. Chow, and N. S. Peachey, "Immunohistochemical studies of the retina following long-term implantation with subretinal microphotodiode arrays," Experimental Eye Research, vol. 73, pp. 333-343, 2001.

[67] M. M. Socha, J. D. Moss, M. Shahin, T. Herndon, J. Wyatt, and J. F. Rizzo, "Mechanical design and surgical implantation of second generation retinal prosthesis," Investigative Ophthalmology \& Visual Science, vol. 40, pp. S735, 1990.

[68] E. de Juan and J. J. Crittenden, "Retinal tacks," Reitna, vol. 3, pp. 2239-42, 1994.

[69] E. de Juan, B. W. McCuen, and R. Machemer, "The use of retina tacks in the repair of complicated retinal detachments," American Journal of Ophthalmology, vol. 102 , pp. $20-24,1986$.

[70] J. U. Meyer, "Retina implant: a bioMEMS challenge," Sensors and Actuators A Physical, vol. 97-98, pp. 1-9, 2002. 


\section{CHAPTER 3: SPECIFICATIONS AND FABRICATION TOOLBOX}

\subsection{INTRODUCTION}

Using electrical stimulation devices to substitute for malfunctioning sensory neural structures are an important bioengineering application that require integrating microelectronic systems with biological systems. Advancements in microfabrication technology provide the tools necessary to conceptualize an intricate, active electronic device small enough to be implanted in the eye. Nevertheless, the production of a highdensity implantable microelectrode array still entails a broad range of development efforts. These efforts include selection of materials, development of fabrication processes, electronic interfacing, and packaging. Implant specifications must be considered when choosing materials and designing the fabrication processes that will produce an implantable system for long-term use. This chapter addresses device requirements and the fabrication toolbox that will be used to meet these requirements.

\subsubsection{Device Requirements}

Table 3.1 lists the desired final device requirements. Long-term biocompatibility is of course vital for the success of any chronically implanted prosthesis. Biocompatibility encompasses biological, materials, mechanical and electrochemical issues. Choosing the appropriate material is crucial especially since this implant will interface with the delicate retinal tissue. Flexibility is a necessary characteristic to allow for surgical manipulation during implant insertion and it permits positioning while minimizing mechanical stress on the retina. At the same time the implant must also be 
robust enough to withstand relatively large deflections and surgical manipulation without itself being damaged. The potential for damage to the delicate retina during abrupt eye movement may indicate the need to use thin, highly conformable soft substrate materials for an implanted array. Chronic inflammation and cellular reactions that occur in response to the physical presence of the foreign body are also of great concern. Responses of immune cells could scar the retinal surface and exert shear forces that could detach the retina. Fabrication development is directed towards minimization of postoperative reactions by using inert materials with a mechanical design that avoids significant tissue distortion. Poly(dimethy siloxane) PDMS is the material of choice for the implant due to its history in medical implants and its mechanical and chemical properties [1].

The ultimate goal for the implant is to have a conformable a $4 \times 4 \mathrm{~mm}$ microelectrode array that interfaces uniformly with the retina. Despite the small size, the implant will also need to contain a fairly large amount of electrodes along with a tack hole for anchoring the device. The aim is to produce an implant that contains one thousand electrodes since the greater the number of electrodes the higher the resolution of the image [2]. Generating an implant that contains one thousand electrodes in $4 \times 4 \mathrm{~mm}$ area is challenging, but achievable with scalable microfabrication technology. However the electrode size is limited by the charge necessary, $1 \mu \mathrm{C}$, to induce visual percept, while minimizing the production of toxic by-products [3]. The main concern with reducing the size of the electrodes will be controlling the charge density in order to prevent the evolution of harmful toxins. This can be accomplished by using balanced biphasic pulses to reverse any toxic reaction and by increasing the surface area of the electrodes to lessen 
the charge density. Also the electrode material must be biocompatible, inert, corrosionresistant, and possess a high charge injection capability. Platinum $(\mathrm{Pt})$ and iridium oxide $\left(\mathrm{IrO}_{2}\right)$ are the prime candidates for the electrode material since they have been proven to exhibit most of these characteristics [4]. For the development of the technology needed to fabricate the final microelectode array, experimental microelectrode array prototypes discussed in chapter 4 and 5 are used to address basic engineering feasibility issues.

Table 3.1. Implant specifications

\begin{tabular}{ll|} 
1. & $4 \mathrm{X} 4 \mathrm{~mm}$ implant \\
2. & Conforms to the retina \\
3. & Scaleable technology for potential 1000 electrode array \\
4. & Electrode material $\mathrm{Pt}$ or $\mathrm{IrO}_{2}$ \\
5. & Charge of $1 \mu \mathrm{C}$ \\
\hline 6. & Tack hole for anchoring capability \\
7. & Mechanically and physiologically biocompatible \\
8. & Mechanically robust to withstand implantation \\
9. & Capability to interface with electronics \\
\hline
\end{tabular}

\subsubsection{Microfabrication Background}

Traditional microfabricated devices use silicon and glass, since the techniques for fabricating in these materials are well developed [5-7]. However, fabrication in silicon and glass requires chemical etching or more complex techniques such as reactive ion etching (RIE) and anodic bonding [7-10]. All of these processes require expensive equipment and are very time-consuming tasks. In addition both silicon and glass are rigid substrates, which makes it difficult to conform to curved delicate tissue such as the retina. In contrast, polymers specifically silicone rubber, offer a number of characteristics that render them suitable for fabrication of an implantable device. In the 
past decade the microfabrication community has integrated the use of polymers, such as PDMS, into its research efforts in order to complement or compensate desired properties not offered by traditional material. Polymers became attractive materials for microfabrication because, per weight, they are inexpensive in comparison to silicon or glass. In addition, their properties can be tailored to meet desired requirements, and they offer rapid prototyping capability $[1,11]$. However, fabrication in polymers is not trivial, but eventually their use as materials can meet design requirements while also reducing the time, complexity, and cost of prototyping and manufacturing $[10,12]$.

Several groups have previously used microfabrication approaches to realize microelectrodes for a variety of applications including neurostimulation. Current materials that are being used as substrates for neural and retinal implant include polyimide and silicon [13-16]. Although microfabrication technology has been well characterized for polyimide and silicon, they both have disadvantageous intrinsic characteristics that will limit there use as long-term implants [15]. . Polyimide absorbs water and lacks the conformabity and softness offered by silicone rubber (see Table 3.2). Silicon is limited due to its mechanical rigidity, fragility, bio-incompatibility. Both of these materials could prove detrimental to long-term implants if not encapsulated prior to insertion with another material such as silicone rubber to render them biocompatible and to protect the electronics from the harsh environment of the eye [15]. Also, if one uses a rigid substrate for the microelectrode array there will inevitably be points on the retina experiencing point-stress/strain. This applied force could lead to scarring or even detachment of the retina [17]. In choosing a material to interface with delicate soft tissue 
such as the retina, one must choose a material with characteristics that resemble that of the tissue. This is important to minimize the stress induced on the tissue.

Table 3.2. Comparison of Young's Modulus of various materials

\begin{tabular}{|l|l|}
\hline Material & Young's Modulus \\
\hline Silicon & $165 \mathrm{GPa}[18]$ \\
\hline Polyimide & $1.3-4 \mathrm{GPa}[19]$ \\
\hline Parylene & $3.2 \mathrm{GPa}[19]$ \\
\hline PDMS & $750 \mathrm{kPa}[9]-3 \mathrm{MPa}[20]$ \\
\hline Hydrogel & $160-290 \mathrm{kPa}[21]$ \\
\hline Brain tissue & $66-250 \mathrm{kPa}[22]$ \\
\hline
\end{tabular}

Our approach is to fabricate the device from PDMS a form of silicone rubber; a material that we believe has great potential for meeting all application requirements. PDMS is an inert biocompatible elastomeric material that has simultaneously low water and high oxygen permeability. In addition, the conformable nature of PDMS is critical for ensuring uniform contact with the curved surface of the retina (see Table 3.2). PDMS is a material that is used in many medical devices, such as contact lens, breast implants, and catheter tubing, and has been demonstrated to withstand the body's chemical and physical conditions without causing adverse side effects [23], suggesting that PDMS may be a favorable material to implant within the eye. Also, PDMS was originally designed for embedding, encapsulating, and insulating delicate electronic circuit components; therefore the implant will be self-packaged to protect the electronics from the harsh environment of the eye. Additional advantages of using PDMS include its low-cost, physical and chemical stability, numerous bonding capabilities and room-temperature processing [9]. 
The fabrication processes that are used in the development of the retinal implant expand upon PDMS molding and bonding techniques developed by the Whitesides' group $[6,24-30]$, adding the ability to photolithographically pattern PDMS films, pattern and deposit metal traces on PDMS films, selectively passivate the traces with a second layer of PDMS, and integrate electronics. This is done using a batch-fabrication approach, which will reduce cost and increase yield. At present, implantable neurostimulation devices such as cochlear implants are painstakingly hand-assembled one at a time from small, conventionally manufactured components.

\subsection{FABRICATION TOOLBOX}

New PDMS fabrication techniques have been developed for the construction of the microelectrode array. The most attractive technique is using photolithography, a planar process, to fabricate these devices. The advantage of using photolithography is that it is a batch fabrication process resulting in maximum output and minimum manual labor, therefore reducing cost. A major limitation to using photolithography is that is an inherently $2 \mathrm{D}$ technique. This characteristic makes it difficult to fabricate devices with features taller than 100 microns, but it is well suited for devices requiring more than one level of fabrication, such as integrated circuits (IC). Three-dimensional features can be created using molding techniques, but this is more time-consuming and requires equipment development for an automated process. Non-photolithographic techniques for fabrication are also discussed as part of the polymer-based microfabrication toolbox. The advantage of non-photolithographic methods is the ability to form $3 \mathrm{D}$ features such as circular channels, which are very difficult to fabricate using planar photolithography. 
The fabrication development toolbox general processes are described below. The original process flow for $1^{\text {st }}$ and $2^{\text {nd }}$ generation devices, complete with detailed process parameters, is located in Appendix 2 and 3 respectively.

\subsubsection{Substrate}

The methods described focus on the exploitation of the physical and chemical properties of the polymer PDMS in the fabrication development. PDMS has been one of the most actively developed polymers for microfluidics $[6,12,26,28,31-33]$. Professor George Whiteside's group at Harvard established the foundation for these polymer techniques [6]. The group developed what is termed "Soft Lithography," a micromolding fabrication technique that utilizes the properties of the silicone rubber PDMS. Some attractive characteristics of PDMS are its chemical and physical properties that make possible the fabrication of devices with useful functionality [34-38] (see Table 3.3). At LLNL, we have developed a polymer-based fabrication capability that expands upon PDMS molding and bonding techniques developed by the Whitesides' group, adding the ability to photolithographically pattern PDMS, pattern metal traces on PDMS film, mold 3D channels, and bond external components such as IC chips and batteries to PDMS. 
Table 3.3. Sylgard ${ }^{\circledR} 184$ (Dow Corning Midland, MI) Characteristics

\begin{tabular}{|c|c|c|}
\hline Property & Characteristic & Application \\
\hline Optical & Transparent; UV cutoff $\sim 240 \mathrm{~nm}[39]$ & $\begin{array}{l}\text { Optical detection form 240- } \\
\text { 1100nm [40] }\end{array}$ \\
\hline Electrical & Dielectric strength $21 \mathrm{kV} / \mathrm{mm}[5,39]$ & Allows for embedding circuits \\
\hline Mechanical & $\begin{array}{l}\text { Elastomeric; tunable Young's modulus, } \\
\text { typical value of } \sim 750 \mathrm{kPa}-3 \mathrm{MPa} \text { and } \\
\text { viscosity is } 4000 \mathrm{mPa} . \mathrm{s}[6,9,20]\end{array}$ & $\begin{array}{l}\text { Conforms to surfaces; allows } \\
\text { actuation by reversible } \\
\text { deformation; stretchable }\end{array}$ \\
\hline Thermal & $\begin{array}{l}\text { Insulating; thermal conductivity } 0.17 \\
\mathrm{~W} / \mathrm{m} . \mathrm{K} ; \text { coefficient of thermal } \\
\text { expansion } 310 \mu \mathrm{m} / \mathrm{m}^{\circ} \mathrm{C}[39]\end{array}$ & $\begin{array}{l}\text { Can be used to insulate heating } \\
\text { elements and solutions; } \\
\text { minimizes dissipation of heat }\end{array}$ \\
\hline Interfacial & $\begin{array}{l}\text { Low surface free energy } \sim 20 \mathrm{erg} / \mathrm{cm}[1 \text {, } \\
41]\end{array}$ & $\begin{array}{l}\text { Allows for easy removal form } \\
\text { mold }\end{array}$ \\
\hline Permeability & $\begin{array}{l}\text { Very low permeability to liquid } \\
\text { water }<0.01 \% \text {. Permeable to gases and } \\
\text { nonpolar organic solvents [39] }\end{array}$ & $\begin{array}{l}\text { Sustains liquid in channels } \\
\text { without leakage. Gasses can be } \\
\text { purged }\end{array}$ \\
\hline Reactivity & $\begin{array}{l}\text { Inert; can be oxidized by exposure to } \\
\text { oxygen plasma }[1,7]\end{array}$ & $\begin{array}{l}\text { Surface can be modified to be } \\
\text { hydrophilic; etching can alter } \\
\text { topography of surface }\end{array}$ \\
\hline Toxicity & Non-toxic [39] & $\begin{array}{l}\text { Can be implanted in-vivo; } \\
\text { supports mammalian cell } \\
\text { growth }[42,43]\end{array}$ \\
\hline
\end{tabular}

The PDMS used for this work is Sylgard ${ }^{\circledR} 184$ from Dow Corning Corporation (Midland, MI) and it comes in two components, a resin and a platinum catalyzed curing agent. Silicon hydride groups present in the curing agent react with the vinyl groups present in the resin to form a cross-linked, elastomeric solid [1]. The PDMS is mixed in a 10:1 mass ratio of resin and curing agent respectively. The two parts are mixed well and degassed under vacuum to dissipate all air-bubbles.

\subsubsection{D Molding}

The viscosity of the PDMS used allows it to be spun onto a substrate or cast into a mold while in its liquid precursor state. As shown in Fig. 3.1 below, the process begins with mold masters that are patterned via photolithography to develop raised or recessed 
features on the surface of the mold substrate as seen in Fig. 3.2. The 10:1 mixture of the PDMS resin and curing agent is stirred thoroughly and degassed under vacuum. Once mixed and thoroughly degassed, the liquid polymer is poured onto the mold and cured under manufacture recommended temperature conditions, conforming to the shape of the master. The cured PMDS is then removed from the mold, resulting in imprints in the polymer surface with high feature resolution, as seen in Fig. 3.3b. Coating the master with gold or silanization of the master facilitates the removal of the PDMS replica after molding without damaging the master or itself. For silanization, the masters can be placed under vacuum for 2 hours with a vial containing several drops of tridecafluoro1,1,2,2-tetrahydrooctyl-1-trichlorosilane [40]. Obtaining a master is the limiting factor in determining the complexity and size of the devices that can be made. Masters can be made in a number of ways including using sacrificial photoresist molds or etching glass or silicon [44]. 


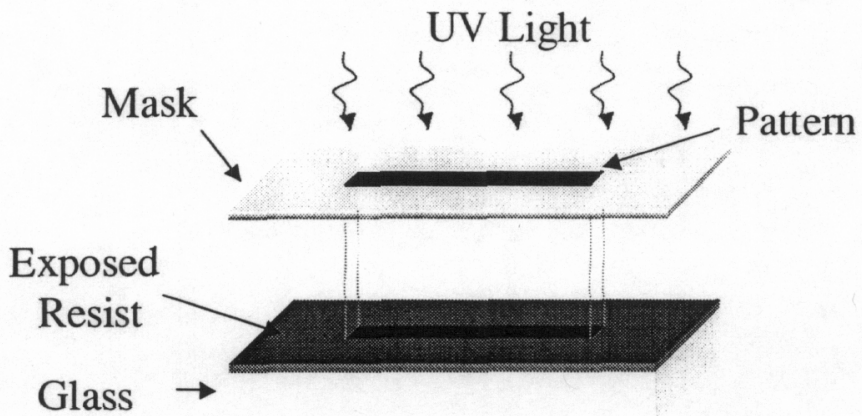

\section{Photolithography}

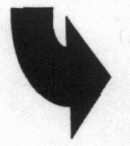

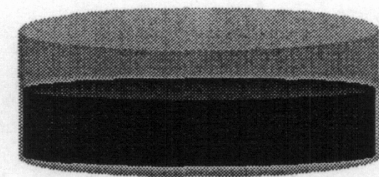

Develop Resist

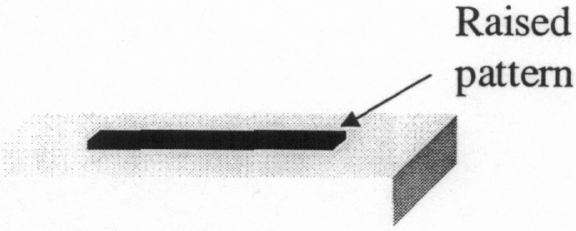

Patterned Resist

Figure 3.1. Photolithography begins by spinning photoresist onto a substrate. Then through a photomask the photoresist is exposed under UV light. Only the protected features will remain while the other areas that were exposed will be washed away during the developing process. This results in patterned photoresist features on the substrate.

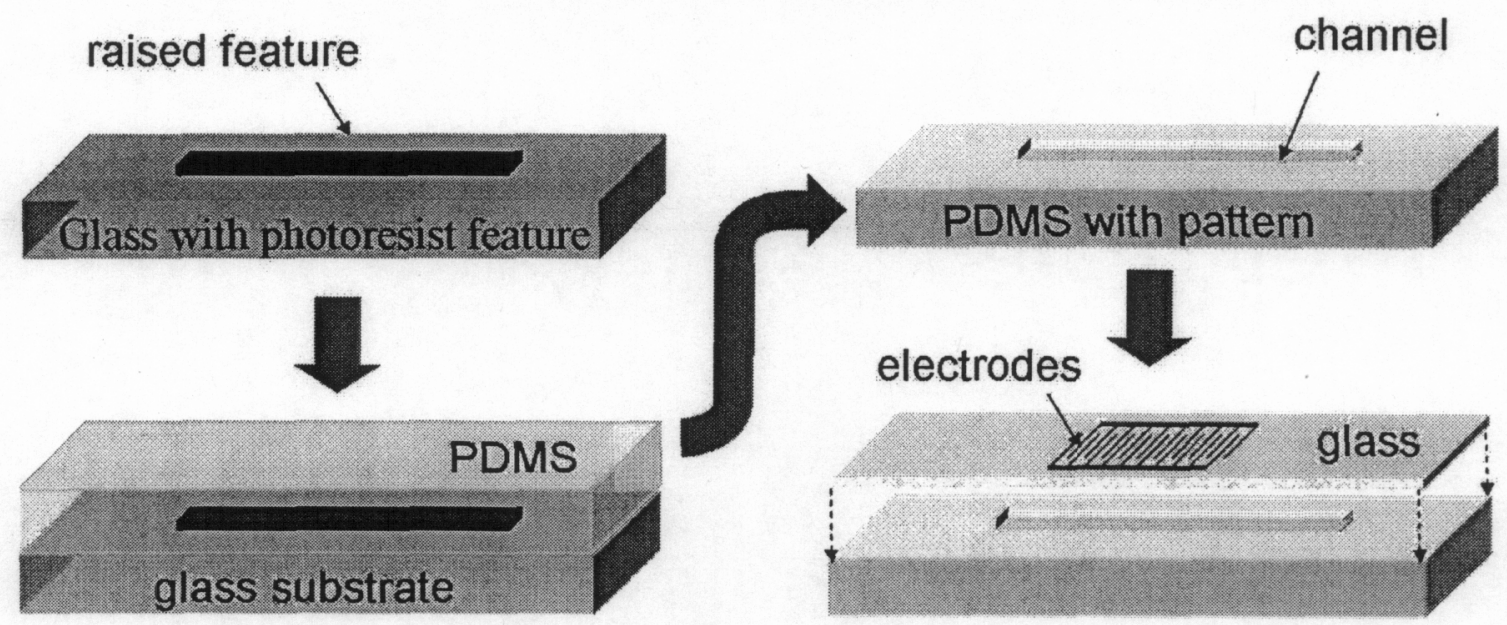

Figure 3.2. Soft Lithography process as developed by the Whitesides' group. Photoresist is patterned on the glass or silicon surface in the desired shape of the channel. PDMS is poured onto the mold and cured. Once removed from the mold, an imprint of the resist creates a channel in the PMDS. A simple bonding procedure is used to seal the channel to PDMS, glass, or silicon [44] b) SEM images of molded PDMS features 


\subsubsection{D Molding}

Soft-lithography has its limitations when producing 3D structures. A suspension molding process allows us to produce $3 \mathrm{D}$ geometries. To produce circular channels, PDMS, while in its liquid precursor state, is poured into a mold frame with suspended wires as shown in Fig. 3.3a. After curing the PDMS, the wires are removed resulting in a circular channel array suspended within the PDMS as seen in the SEM in Fig. 3.3a. Other 3D structures can be fabricated in a similar manner.
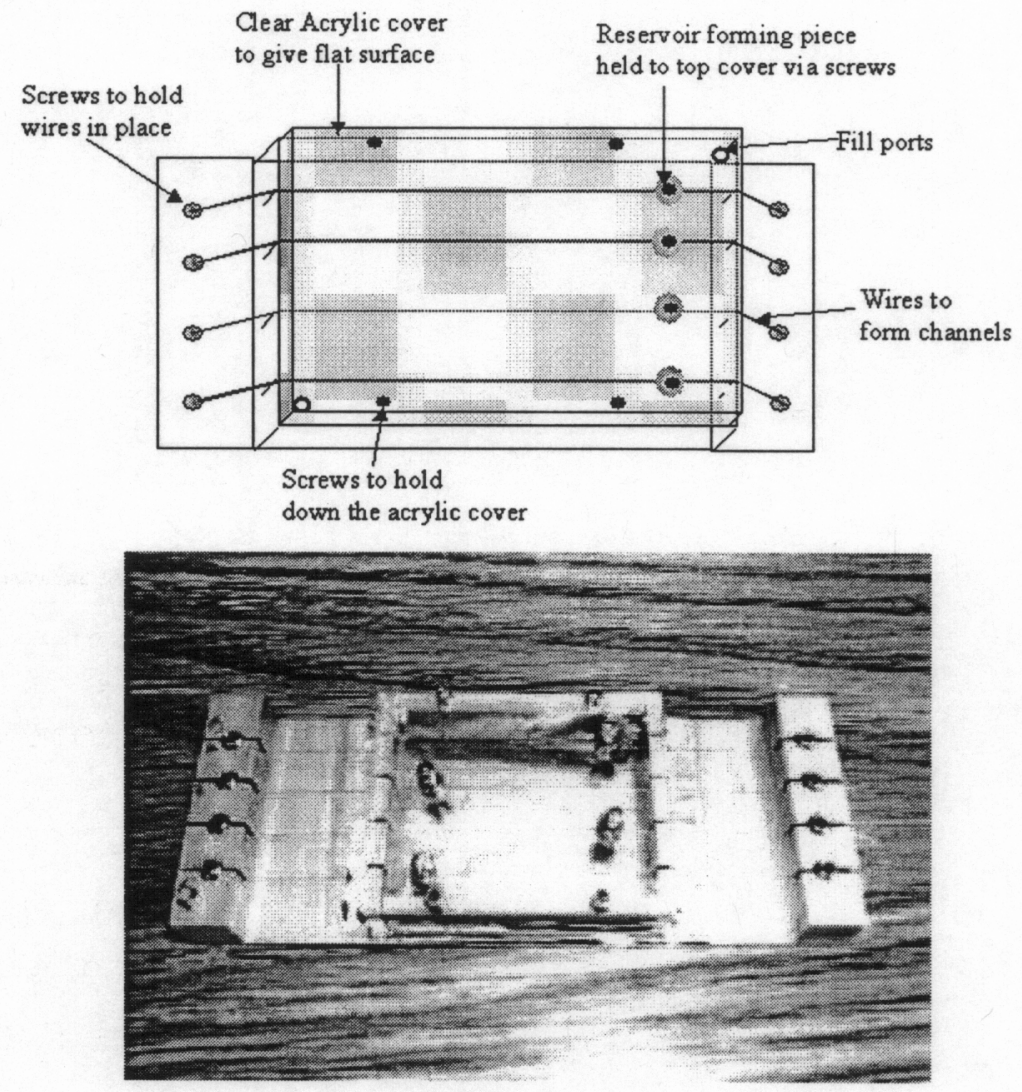


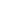


contaminants from the surfaces of both materials with an ethanol rinse, then oxidizing the bonding surfaces in an RF facilitated oxygen plasma to activate their surface chemistry $[12,32,41]$. After the oxygen plasma exposure, the PDMS is temporarily rendered hydrophilic. The treatment generates $(\mathrm{Si}-\mathrm{OH})$ on the surface of the PDMS by the oxidation of methyl groups $[1,11]$. The two materials are then removed from the plasma and placed in conformal contact to create an instantaneous bond. This process generates an irreversible bond with strength greater than that of the bulk material [44]. Surfaceoxidized PDMS has been shown to bond to itself, glass, silicon, polyethylene, polystyrene, silicon nitride [9], and certain metals such as titanium and chromium. The oxygen plasma oxidizes the surface resulting in an increase in surface energy, which allows for lower energy reactions to easily occur, due to free energy effects [45].

Although the bonding process is simple and reproducible it requires technical agility. Following the oxygen plasma the two surfaces must be brought into contact quickly (within approximately one minute) in order to form the best bond, because the surface of the PDMS reconstructs within one hour [5, 7]. Its reported that contact with water or polar organic solvents maintains the hydrophilic nature of the surface indefinitely [40]. Also, our group at LLNL determined that storage in a nitrogen rich environment helps maintain the hydrophilic surface [46]. Experimental evidence shows that the oxidative bonding works best when the samples and chamber are clean and dry and the surfaces are smooth on the micron scale [40]. To refresh an aged PDMS surface for bonding, a hydrochloric acid $(\mathrm{HCl})$ treatment is performed prior to the ethanol rinse and plasma treatment. Applying vacuum and heating has been shown to strengthen a weak bond and removes any trapped air-bubbles that might be present[47]. 
If alignment needs to be performed before bonding, a number of delayed bonding strategies are available [11]. The alignment of two oxidized layers of PDMS can be difficult since the bonding of the two pieces occur on contact. Quake et al. have developed an alternative method for irreversibly sealing that involves adding an excess of the resin to one layer of PDMS and excess curing agent to the other layer [8]. When these layers are brought into contact and cured, the seal that formed was indistinguishable in physical properties from bulk PDMS [7, 8]. The method allows for careful alignment of two pieces of PDMS since the bonding process is initiated by heat, but this method is limited to only devices consisting of PDMS. Another technique that is used for alignment is the use of a solvent between the layers $[28,40]$. The oxidized surfaces are completely immersed in a polar solvent such as methanol immediately after removing the PDMS from the oxygen plasma $[5,40]$. The solvent acts in three ways: 1) It prevents instantaneous sealing when two layers are brought into contact; 2) It provides lubrication and allows the layers to be moved laterally relative to one another; 3) It prevents the oxidized surface from reconstructing to a lower free-energy state before bonding is accomplished. Evaporation of the methanol is achieved by placing the part directly onto a hotplate or in an oven following alignment, and a permanent bond between the two materials is achieved. Bonding with this method produces a bond equivalent to bonding immediately upon removal from the oxygen plasma [5]. Another method of alignment is utilizing a set-up similar to a mask aligner, where one part is fixed and the other part is placed on a moving stage in close proximity to the first part, but not in contact. Prior to alignment the surfaces are activated using RF facilitated oxygen plasma. Using a microscope, the two parts are aligned and then brought into contact for the bond to form. 
Following the bonding process the parts are placed under vacuum to ensure removal of any trapped air between the two parts.

\subsubsection{Patterning PDMS}

As an alternative to etching and using other materials, a polymer patterning technique developed by Duffy et al. [10] was experimented with. The group used SU-8 photoresist to create $50 \mu \mathrm{m}$ tall posts with $50 \mu \mathrm{m}$ diameters on a silicon substrate. They then spun on a layer of PDMS with a thickness below the height of the posts. The PDMS was cured and the film was removed from the silicon substrate. This PDMS film was implemented as a reusable "dry liftoff" mask for patterning. The results from their experiments showed precisely pattern columns with $50 \mu \mathrm{m}$ diameters in a PDMS. These results generated interest in the applicability of this approach because it is a potential means for photolithographically patterning PDMS films. Instead of using permanent posts and removing the PMDS film, the process is modified to remove the photoresist features from the substrate leaving a patterned PDMS film for subsequent processing steps, as seen in Fig. 3.4. Alignment would be nearly impossible and the process would be labor intensive if the PDMS film is lifted from the molding substrate, and then bonded to substrate. But it would be very precise if the photoresist is removed instead allowing for further processing steps to take place. 
(1)

(2)

(3)
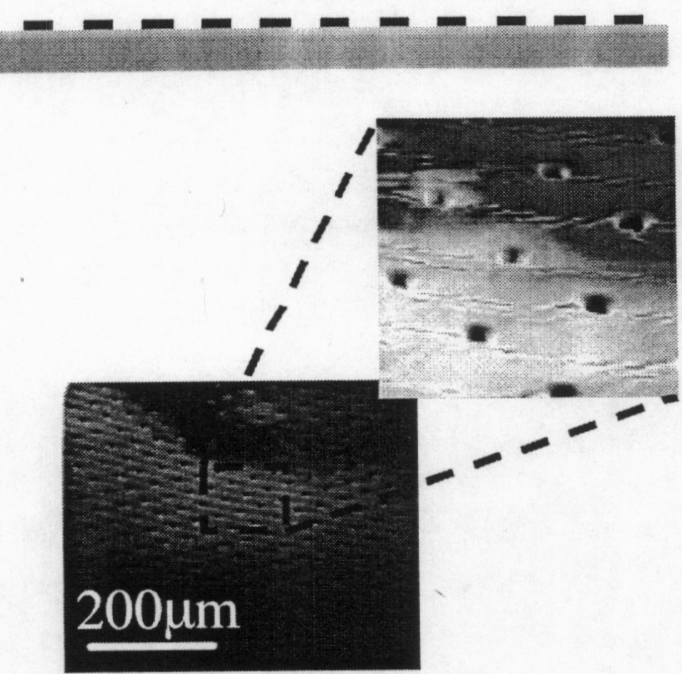

(4)

(5)

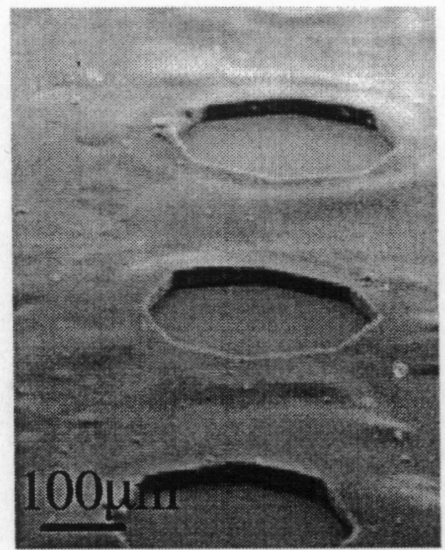

Figure 3.4. PDMS patterning 1) Start with handle wafer 2) Coat handle wafer with gold to prevent PDMS from permanently bonding to the wafer 3) Pattern photoresist using photolithography 4) Spin PDMS and allow to planarize 5) Cure PDMS and remove photoresist. SEM images illustrate patterned PDMS.

While in its liquid precursor state, PDMS films were spun onto gold-coated silicon wafers at spin rates varying from 1000 to $7000 \mathrm{rpm}$ for 20 and 90 seconds producing of PDMS films from $4 \mu \mathrm{m}$ to $70 \mu \mathrm{m}$ thick. The gold surface prevents the PDMS from permanently adhering to the substrate. The PDMS thickness was measured using a Tencor ${ }^{\circledR}$ P-10 stylus profilometer and the resulting spin-rate chart is shown in Fig. 3.5. To pattern the PDMS, a sacrificial thick photoresist layer (AZ ${ }^{\circledR} \mathrm{PLP}$ 100XT, Clariant) was patterned on the surface using photolithography. The resist was patterned to create a reverse image of the desired polymer structure on the substrate. These features were approximately $60 \mu \mathrm{m}$ thick to allow for relatively thick polymer layers. PDMS was spun onto the surface at a thickness less than the height of the photoresist 
columns and allowed to planarize, then cured. After curing, removal of photoresist produced open regions in the PDMS film, as shown in Fig. 3.4.

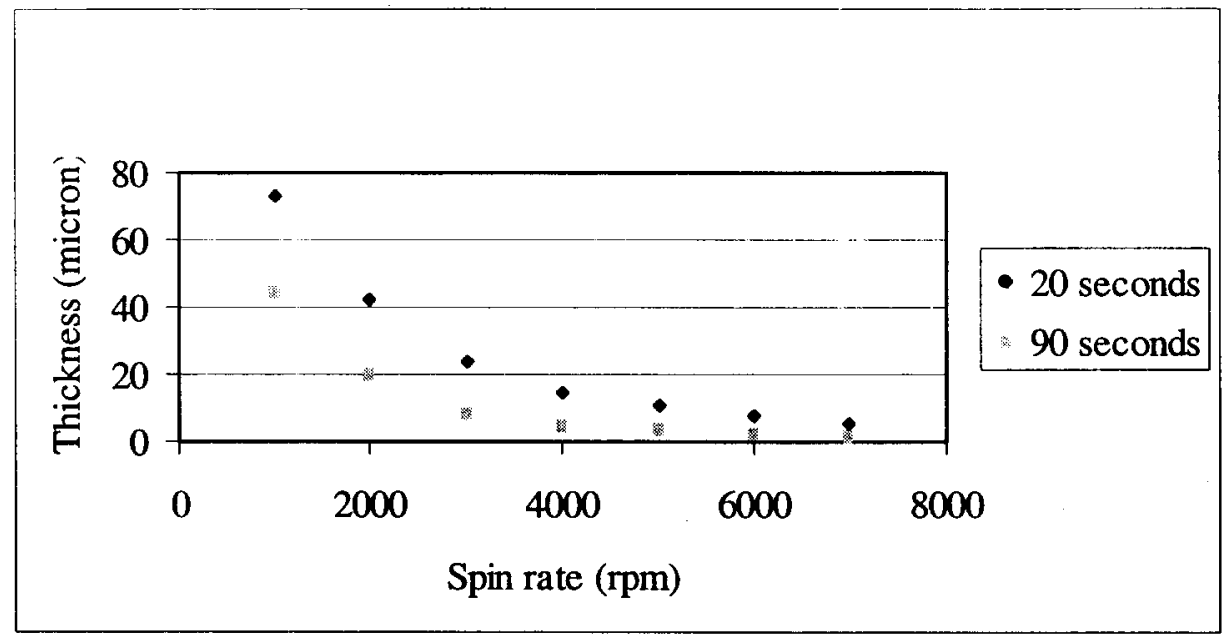

Figure 3.5. PDMS thickness as a function of spin rate at 20 and 90 seconds.

\subsubsection{Metalizing PDMS}

Gold leads are patterned on top of PDMS films using a lift-off process. An oxygen plasma treatment of the PDMS is necessary prior to metal evaporation to promote the adhesion of metal to the active PDMS surface. Photoresist is spun onto the film surface and patterned using traditional photolithographic techniques. Prior to photoresist application, the wafer is placed in an oxygen plasma to oxidize the surface. This allowed the resist to wet the PDMS surface, eliminating beading and ensuring the formation of a smooth and uniform coat of photoresist on the polymer surface. A gold film is then ebeam evaporated onto the wafer using titanium or chromium as the adhesion layer [24]. The metal adheres to the PDMS surface in developed regions in the photoresist and the excess is removed through a lift-off process by placing the wafer in acetone as seen in 
Fig. 3.6. The wafer is then prepared for passivation by rinsing with ethanol and drying gently.

(1)

(2)

(3)
(4)

(5)

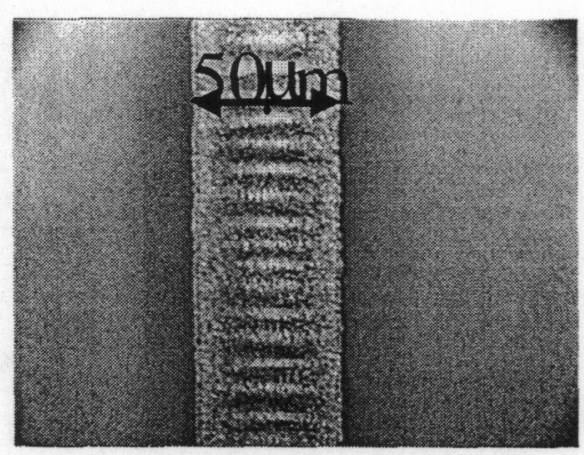

Figure 3.6. PDMS metalization using lift-off process 1) Start with handle wafer 2) Coat the wafer with gold 3) Spin PDMS onto the wafer 4) Spin photoresist on oxidized surface, pattern and re-oxidize the exposed surface 5) evaporate metal film and Lift-off

\subsubsection{Selective Passivation Layer}

A passivation layer is required on the patterned electrodes to avoid shorting of the electrical circuits when implanted. PDMS is also the material of choice for the passivation layer for both its insulating properties [39] and its water resistance. The electrical insulation on the leads must reliably prevent levels of leakage current that would be harmful physiologically and prevent materials from seeping in and degrading the implant in vivo. A thin layer of PDMS ranging from 7 to $20 \mu \mathrm{m}$ is used to passivate the device. An oxygen plasma treatment needs to be performed before passivation to ensure adhesion of the second PDMS layer. There are three methods that can be used in 
passivating the devices. One is using a stencil-like mask that is placed on the surface of the first PDMS layer, and the second PDMS layer is spun onto the substrate. The stencil is then removed prior to curing, exposing regions of the traces necessary for electrical connections as seen in Fig. 3.7. The second and third methods involve PDMS patterning. Method two requires patterning thick photoresist (Clariant's PLP 100XT) on top of the first layer of metalized PDMS. The photoresist remains in the regions where electrical connections will be made. PDMS is then spun onto the first layer over the phororesist and allowed to planarize prior to curing. After curing the photoresist is removed, exposing the metal in the desired regions. The third method involves patterning PDMS onto a flexible substrate such as polyimide and performing a membrane transfer onto the original substrate for passivation. This is a lamination process. Photoresist is first patterned onto the flexible substrate then PDMS is spun onto the substrate, planarized and cured. The photoresist is then removed leaving a patterned PDMS film onto the flexible substrate. Both the original substrate with metalized PDMS film and the flexible substrate with patterned PDMS film are rinsed with ethanol and placed in the oxygen plasma to activate the surface for bonding. Alignment bonding is used to line up the two films and then they are brought into contact. Once in contact they are placed under vacuum to remove any trapped air-bubbles. Next, the flexible substrate is peeled back resulting in the selective passivation of the metal traces. 
(1)

(2)

(3)

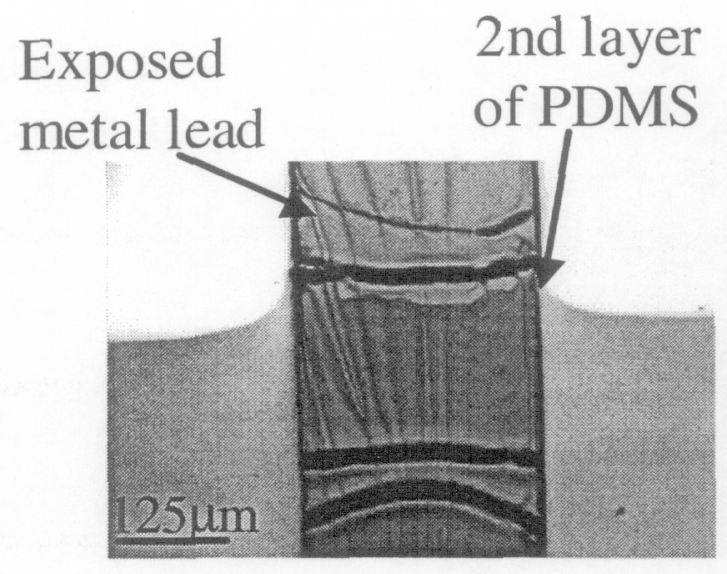

(4)

(5)
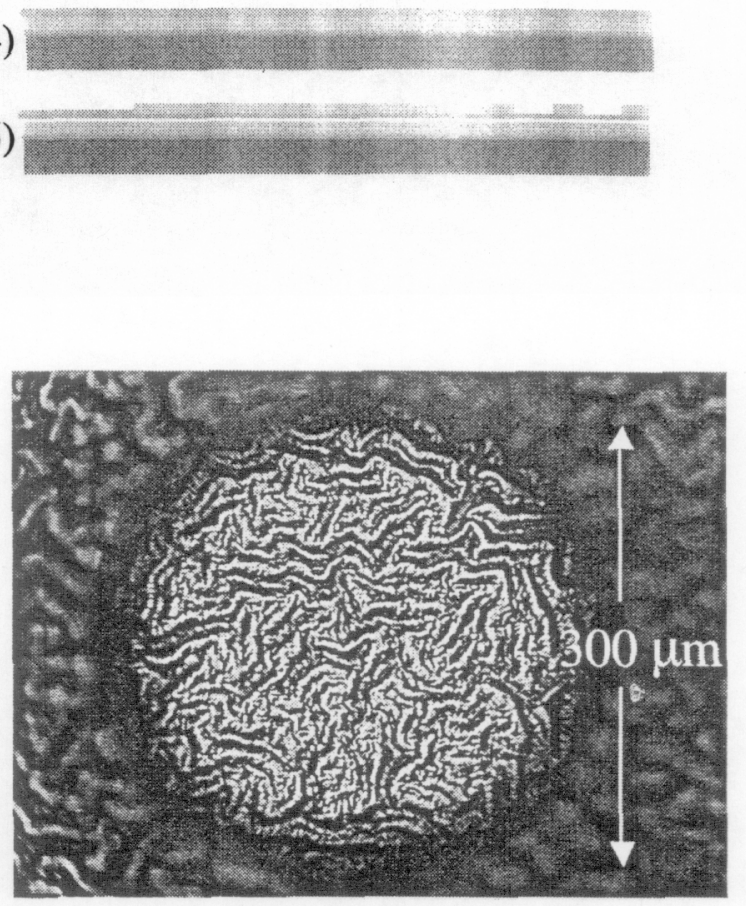

Figure 3.7. Selectively Passivating metalized PDMS 1) Start with handle wafer 2) Coat handle wafer with gold to prevent PDMS from permanently bonding to the wafer 3) Spin PDMS and cure 4)Metalize PDMS 5) Selectively passivate PDMS. Optical images illustrate passivated PDMS with exposed metal for electrical connection.

\subsubsection{Integrating External Components}

Components such as valves $[8,48-50]$, pumps $[8,12,50]$,electronic components [51, 52], and sensors are needed to develop complete systems [25]. These components can be integrated either through bonding or encapsulation within PDMS. Integrating the electronic chip with the electrode array is predicted to be one of most challenging aspects of the project. One of the primary obstacles to long-term chronic implants in the past has been the absence of a satisfactory means for connecting the electrodes to the outside world. Even when implantable telemetry systems are used for transmission of data and power, leads are required between the electrodes and the electronics package. Utilizing LLNL's polymer-based fabrication toolbox we demonstrated the capability of 
irreversibly bonding silicon electronics chips to PDMS, as shown in Fig. 3.8. Developing a reliable means to interface the implant to the outside world is also crucial for reliable and repeatable testing purposes.
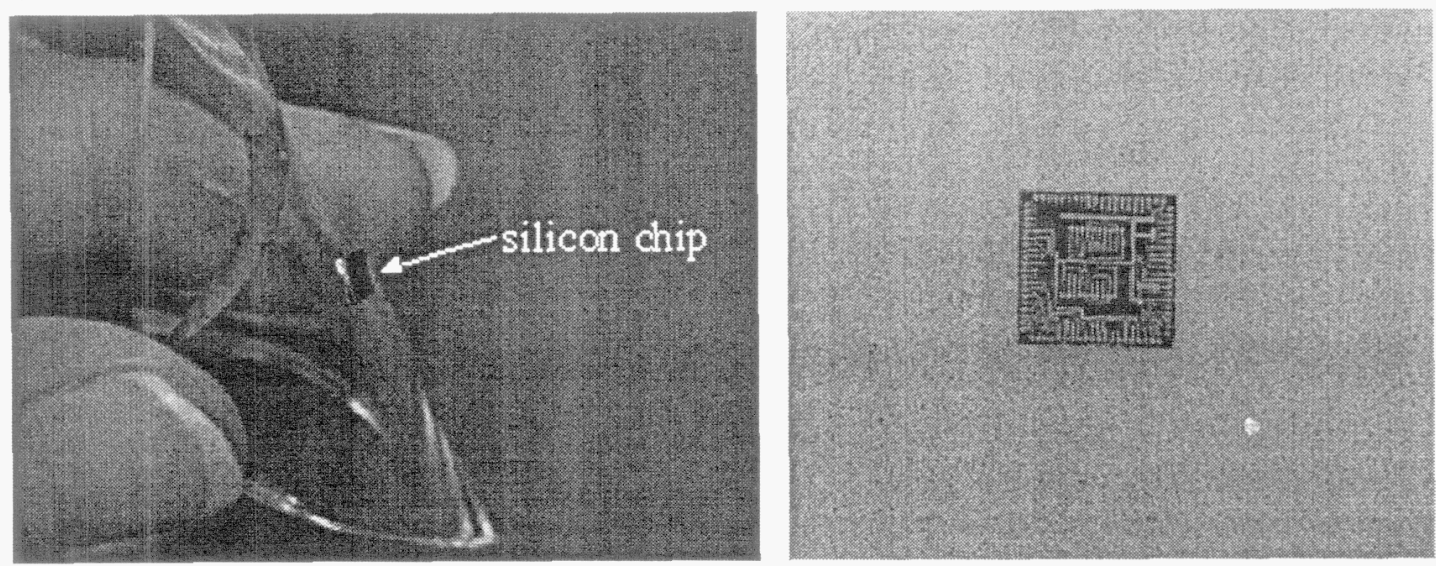

Figure 3.8. The image on the left shows a silicon chip irreversibly bonded to PDMS. On the right is an image of the bonded silicon chip looking through the PDMS.

\subsection{DISCUSSION}

Providing sight to the visually impaired through retinal implants would be a phenomenal application of microtechnology. In the field of microfluidics PDMS has been shown to be particularly useful in prototyping new systems $[5,12,26,28,37,40$, 44]. As the focus shifts from microfluidics components to the developments of other devices such as implantable microelectrode array new fabrication techniques were required. PDMS offers many attractive characteristics such as conformability, biocompatibility and the ability to bond to itself and other materials, which makes it a great candidate for developing the implantable microelectrode array for the retinal. prosthesis. 


\subsection{REFERENCES}

[1] S. J. Clarson and J. A. Semlyen, Siloxane polymers. Englewoood Cliffs, N.J.: Prentice Hall, 1993.

[2] M. Humayun, "Intraocular Retinal Prosthesis," Tr. Am. Ophth. Soc, vol. 99, pp. 271-300, 2001.

[3] A. Hung, D. Zhou, R. Greenberg, and J. Judy, "Micromachined Electrodes for High Density Neural Stimulation Systems," presented at IEEE special topics in medicine and biology, Madison, WI, 2002.

[4] W. T. Liu and M. Humayun, "Artificial Retinal Prosthesis to Restore Vision for the Blind," presented at Digest of the LEOS Summer Topical Meetings, 2000.

[5] B. H. Jo, L. M. Van Lerberghe, K. M. Motsegood, and D. J. Beebe, "Threedimensional micro-channel fabrication in polydimethylsiloxane (PDMS) elastomer," Journal of Microelectromechanical Systems, vol. 9, pp. 76-81, 2000.

[6] G. M. Whitesides, "Unconventional methods and unconventional materials for microfabrication," 1997.

[7] S. R. Quake and A. Scherer, "From micro- to nanofabrication with soft materials," Science, vol. 290, pp. 1536-1540, 2000.

[8] M. A. Unger, H. P. Chou, T. Thorsen, A. Scherer, and S. R. Quake, "Monolithic microfabricated valves and pumps by multilayer soft lithography," Science, vol. 288, pp. 113-116, 2000.

[9] D. Armani, C. Liu, and N. Aluru, "Re-configurable fluid circuits by PDMS elastomer micromachining," 1999.

[10] G. Kovacs, Micromachined Transducers Sourcebook. New York: McGraw-Hill, 1998.

[11] E. P. Everaert, H. C. Van der Mei, and H. J. Busscher, "Hydrophobic recovery of repeatedly plasma-treated silicone rubber. 2. A comparison of the hydrophobic 
recovery in air, water, or liquid nitrogen," Journal of Adhesion Science and Technology, vol. 10, pp. 351-9, 1996.

[12] P. Krulevitch, W. Bennett, J. Hamilton, M. Maghribi, and K. Rose, "Polymerbased packaging platform for hybrid microfluidic systems," Biomedical Microdevices, vol. 4, pp. 301-308, 2002.

[13] J. F. Rizzo, J. Wyatt, M. Humayun, E. de Juan, W. T. Liu, A. Chow, R. Eckmiller, E. Zrenner, T. Yagi, and G. Abrams, "Retinal prosthesis - An encouraging first decade with major challenges ahead," Ophthalmology, vol. 108, pp. 13-14, 2001.

[14] E. Zrenner, "The subretinal implant: Can microphotodiode arrays replace degenerated retinal photoreceptors to restore vision?," Ophthalmologica, vol. 216, pp. 8-20, 2002.

[15] J. U. Meyer, "Retina implant: a bioMEMS challenge," Sensors and Actuators A Physical, vol. 97-98, pp. 1-9, 2002.

[16] A. Y. Chow, M. T. Pardue, J. I. Perlman, S. L. Ball, V. Y. Chow, J. R. Hetling, G. A. Peyman, C. P. Liang, E. B. Stubbs, and N. S. Peachey, "Subretinal implantation of semiconductor-based photodiodes: Durability of novel implant designs," Journal of Rehabilitation Research and Development, vol. 39, pp. 313$321,2002$.

[17] D. Edell, J. F. Rizzo, and J. Wyatt, "Low-pressure Neural contact structure," in US Patent 5,575,813, 1996.

[18] M. Madou, Fundamentals of Microfabrication. New York: CRC, 1997.

[19] MatWeb, "The online materials database," vol. 2003, 2003.

[20] H. Schmid and B. Michel, "Siloxane polymers for high-resolution, high-accuracy soft lithography," Macromolecules, vol. 33, pp. 3042-3049, 2000.

[21] B. Johnson, D. J. Niedermaier, W. C. Crone, J. Moorthy, and D. Beebe, "Mechanical properties of $\mathrm{pH}$ sensitive hydrogel," presented at Society for experimental mechanics, SEM Annual Conference Proceedings, Milwaukee, WI, 2002. 
[22] T. Aida, "Study of human head impact: brain tissue constitutive models," in Mechanical Engineering. Morgantown, West Virginia, 2000.

[23] F. H. Silver and D. L. Christiansen, Biomaterials Science and Biocompatibility. New York: Springer-Verlag, 1999.

[24] N. Bowden, S. Brittain, A. G. Evans, J. W. Hutchinson, and G. Whitesides, "Spontaneous formation of ordered structures in thin films of metals supported on a elastomeric polymer," Nature, vol. 393, pp. 146-149, 1998.

[25] I. A. Aksay, J. T. Groves, S. M. Gruner, P. C. Y. Lee, R. K. Prud'homme, W. H. Shih, S. Torquato, and G. M. Whitesides, "Smart materials systems through mesoscale patterning," 1996.

[26] J. R. Anderson, D. T. Chiu, R. J. Jackman, O. Cherniavskaya, J. C. McDonald, H. $\mathrm{K}$. Wu, and G. M. Whitesides, "Fabrication of topologically complex threedimensional microfluidic systems in PDMS by rapid prototyping," Analytical Chemistry, vol. 72, pp. 3158-3164, 2000.

[27] S. Brittain, K. Paul, X. M. Zhao, and G. Whitesides, "Soft lithography and microfabrication," Physics World, vol. 11, pp. 31-36, 1998.

[28] D. C. Duffy, J. C. McDonald, O. J. A. Schueller, and G. M. Whitesides, "Rapid prototyping of microfluidic systems in poly(dimethylsiloxane)," Analytical Chemistry, vol. 70, pp. 4974-4984, 1998.

[29] R. J. Jackman, S. T. Brittain, A. Adams, M. G. Prentiss, and G. M. Whitesides, "Design and fabrication of topologically complex, three-dimensional microstructures," Science, vol. 280, pp. 2089-91, 1998.

[30] J. C. McDonald, S. J. Metallo, and G. M. Whitesides, "Fabrication of a configurable, single-use microfluidic device," Analytical Chemistry, vol. 73, pp. 5645-5650, 2001.

[31] E. Delamarche, A. Bernard, H. Schmid, A. Bietsch, B. Michel, and H. Biebuyck, "Microfluidic networks for chemical patterning of substrate: Design and application to bioassays," Journal of the American Chemical Society, vol. 120, pp. 500-508, 1998. 
[32] J. C. McDonald, D. C. Duffy, J. R. Anderson, D. T. Chiu, H. K. Wu, O. J. A. Schueller, and G. M. Whitesides, "Fabrication of microfluidic systems in poly(dimethylsiloxane)," Electrophoresis, vol. 21, pp. 27-40, 2000.

[33] G. M. Whitesides and A. D. Stroock, "Flexible methods for microfluidics," Physics Today, vol. 54, pp. 42-48, 2001.

[34] W. S. Beh, I. T. Kim, D. Qin, Y. N. Xia, and G. M. Whitesides, "Formation of patterned microstructures of conducting polymers by soft lithography, and applications in microelectronic device fabrication," Advanced Materials, vol. 11, pp. 1038-1041, 1999.

[35] J. M. Ho, R. G. Beck, R. M. Westervelt, and G. M. Whitesides, "The use of soft lithography to fabricate arrays of Schottky diodes," Advanced Materials, vol. 10, pp. 574t, 1998.

[36] J. C. Love, J. R. Anderson, and G. M. Whitesides, "Fabrication of threedimensional microfluidic systems by soft lithography," MRS Bulletin, vol. 26, pp. 523-8, 2001.

[37] Y. N. Xia and G. M. Whitesides, "Soft lithography," Annual Review of Materials Science, vol. 28, pp. 153-184, 1998.

[38] G. M. Whitesides, E. Ostuni, S. Takayama, X. Y. Jiang, and D. E. Ingber, "Soft lithography in biology and biochemistry," Annual Review of Biomedical Engineering, vol. 3, pp. 335-373, 2001.

[39] Dow Corning Corporation, "Sylgard 184," Midland MI 2002.

[40] J. C. McDonald, "Analytical applications of elastomeric microfluidic devices," in Chemistry and Chemical Biology. Cambridge: Harvard University, 2001.

[41] D. Eon, L. de Poucques, M. C. Peignon, C. Cardinaud, G. Turban, A. Tserepi, G. Cordoyiannis, E. S. Valamontes, I. Raptis, and E. Gogolides, "Surface modification of Si-containing polymers during etching for bilayer lithography," Microelectronic Engineering, vol. 61-62, pp. 901-6, 2002.

[42] G. P. Lopez, M. W. Albers, S. L. Schreiber, R. Carroll, E. Peralta, and G. M. Whitesides, "Convenient Methods for Patterning the Adhesion of Mammalian 
Cells to Surfaces Using Self-Assembled Monolayers of Alkanethiolates on Gold," Journal of the American Chemical Society, vol. 115, pp. 5877-5878, 1993.

[43] E. Ostuni, R. Kane, C. S. Chen, D. E. Ingber, and G. M. Whitesides, "Patterning mammalian cells using elastomeric membranes," Langmuir, vol. 16, pp. 7811$7819,2000$.

[44] K. Rose, "A programmable microfluidic Processor: Integrated and hybrid solutions," in Mechanical Engineering. Cambridge: Massachusetts Institute of Technology, 2002, pp. 114.

[45] M. Ohring, Material Science of Thin Films: Deposition \&Structure, Second Edition ed. San Diego: Academic Press, 2002.

[46] C. Davidson and A. Tovar, "Nitrogen rich environment to maintain fresh PDMS surface." Livermore, 2002.

[47] J. Hamilton, "Appling vacuum and heat to remove air-bubbles." Livermore, 2002.

[48] A. G. MacDiarmid, H. L. Wang, F. Huang, J. K. Avlyanov, P. C. Wang, T. M. Swager, Z. Huang, A. J. Epstein, Y. Wang, D. D. Gebler, R. Shashidhar, J. M. Calvert, R. J. Crawford, T. G. Vargo, G. M. Whitesides, Y. Xia, and B. R. Hsieh, "Application of thin films of conjugated polymers in novel LED's and liquid crystal "light valves"," 1996.

[49] V. Namasivayam, R. H. Liu, B. Towe, and P. Grodzinski, "Design of a selfcontained 3D microvalve in PDMS," 2001.

[50] J. Noo Li, D. T. Chiu, C. J. Wargo, W. Hongkai, I. S. Choi, J. R. Anderson, and G. M. Whitesides, "Design and fabrication of integrated passive valves and pumps for flexible polymer 3-dimensional microfluidic systems," Biomedical Microdevices, vol. 4, pp. 117-21, 2002.

[51] H. Junmin, D. Tao, R. G. Beck, R. M. Westervelt, and G. M. Whitesides, "Fabrication of arrays of Schottky diodes using microtransfer molding," Sensors and Actuators A (Physical), vol. A75, pp. 65-9, 1999. 
[52] M. Maghribi, J. Hamilton, D. Polla, K. Rose, T. Wilson, and P. Krulevitch, "Stretchable Micro-Electrode Array for Retinal Prosthesis," presented at IEEEEMBS special topics n medicine and biology, Madison, WI, 2002. 


\section{CHAPTER 4: $1^{\text {ST }}$ GENERATION DEVICES}

\subsection{INTRODUCTION}

This chapter addresses the $1^{\text {st }}$ generation process flow design, fabrication, and testing. The $1^{\text {st }}$ generation devices are comprised of three different feasibility prototypes. Prototype-1 is for developing the platinum electroplating process and it consists of patterned PDMS film on gold-coated silicon wafer. The gold on the silicon substrate, as illustrated in Fig. 4.1a, serves as the seed layer for the electroplating process. Prototype-2 consists of metal traces sandwiched between two PDMS layers on a bare silicon substrate, as illustrated in Fig. 4.1b. Prototype- 2 is for conducting soak tests. Prototype3 also contains metal traces sandwiched in between two PDMS layers, however this prototype is removed from the handle wafer after fabrication, Fig. 4.1c. This prototype is for surgical handling and conformability evaluation. Using separate prototypes for various experiments allows for parallel fabrication development without having a problematic step hampering the entire effort. These $1^{\text {st }}$ generation experimental prototypes are designed according to our collaborator's, at the University of Southern California (USC), specifications. The' techniques discussed in chapter 3 are used in generating these prototypes, which will ultimately lead to a process flow to meet the overall microelectrode array design specifications (See chapter 3). Integrating various aspects of the feasibility prototypes leads to a $1^{\text {st }}$ generation microelectrode array. An overall design plan for achieving the $1^{\text {st }}$ generation microelectrode array is presented below. 


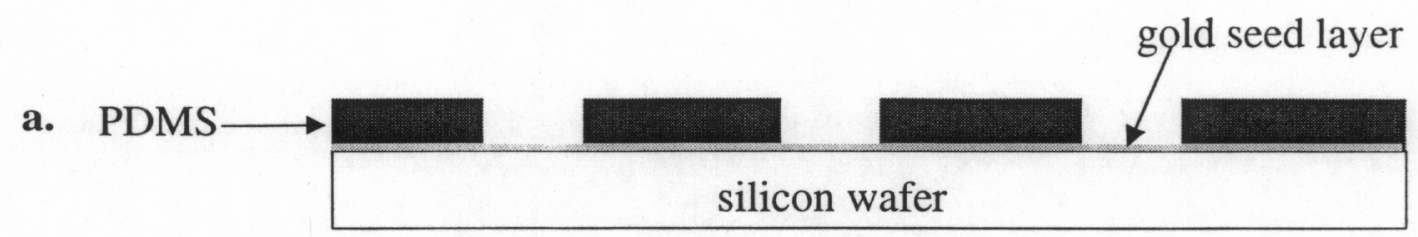

Side View

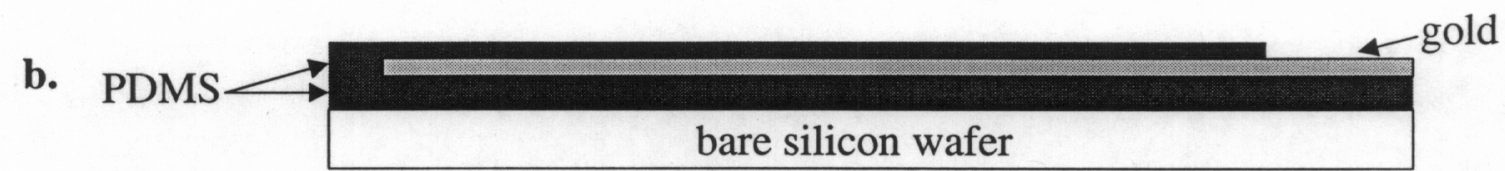

Side View

c.

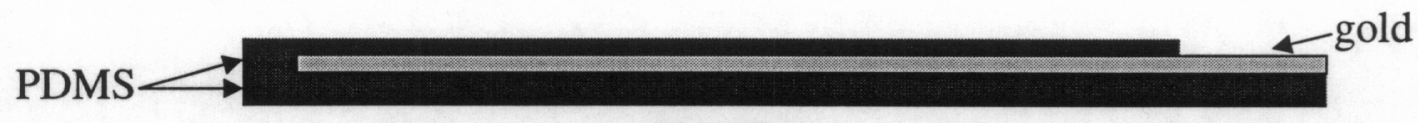

Side View

Figure 4.1. $1^{\text {st }}$ generation feasibility prototypes a) prototype-1 electrode development, b) prototype-2 soak testing, c) prototype- 3 implantation

\subsection{1 $1^{\text {st }}$ Generation Microelectrode Array Fabrication Process Plan}

The $1^{\text {st }}$ generation microelectrode array process flow design plan is illustrated in Fig. 4.2. This is a batch fabrication process. This means multiple devices are generated on each wafer simultaneously after the completion of a series of steps. The $1^{\text {st }}$ generation microelectrode array fabrication process design begins with a four-inch gold-coated silicon wafer. The gold film serves as a seed layer for electroplating the electrodes. The gold film also facilitates the removal of the polymer from the wafer. Thick photoresist is spun onto the handle wafer and baked. The photoresist is UV exposed using a photomask. The pattern is developed, as illustrated in Fig. 4.2a, and the PDMS is applied at a spin rate that produces a film thickness below the photoresist feature height, Fig. 4.2b. Once the PDMS is cured, the photoresist is striped, Fig. 4.2c, using the appropriate 
solvents. Platinum is electroplated in the PDMS patterned holes to form an array of electrical stimulators, Fig. 4.2d. After electroplating through the patterned areas in the PDMS film, thick photoresist is again spun onto the PDMS surface and baked. Prior to photoresist application, the wafer is placed in an oxygen plasma to oxidize the surface. The photoresist features are exposed and developed as previously described and placed for a second time in the oxygen plasma to activate the newly exposed PDMS surface, and promote adhesion of the metal. A gold film is e-beam evaporated onto the wafer using titanium as the adhesion layer. The metal is expected to adhere to the PDMS surface in developed regions in the photoresist and the excess is removed through a lift-off process by placing the wafer in acetone, Fig. 4.2e. The wafer is then prepared for passivation by rinsing with ethanol and drying gently. An oxygen plasma treatment is again performed before passivation to ensure adhesion of the second PDMS layer. A stencil-like mask is then placed on the metal traces, and the second PDMS layer is spun onto the substrate. The stencil is removed prior to curing, exposing regions of the traces necessary for electrical connections, Fig. 4.2f. After the PDMS is cured the wafer is diced and the devices are removed from the wafer exposing the platinum electrodes, Fig. $4.2 \mathrm{~g}$. 

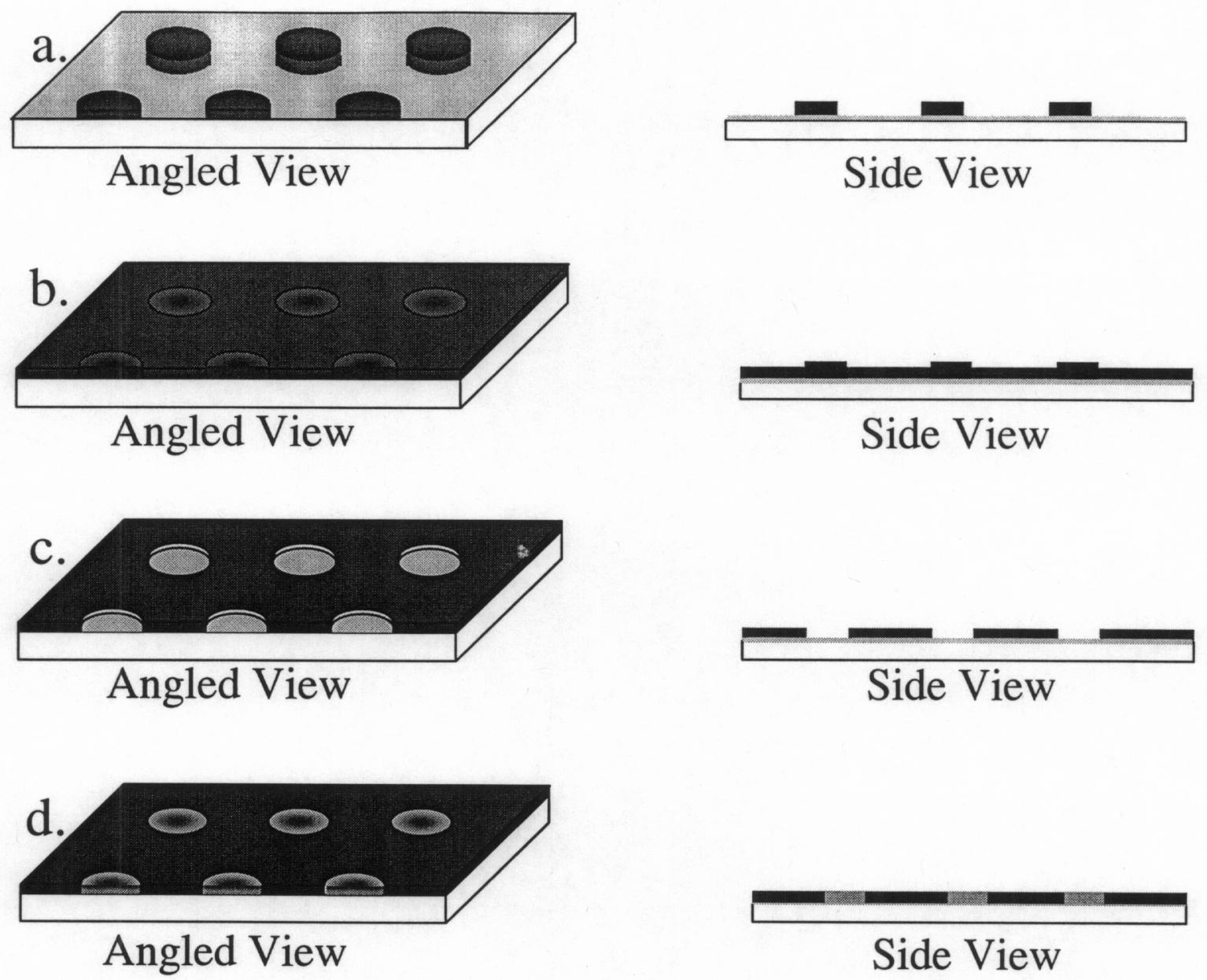

Side View
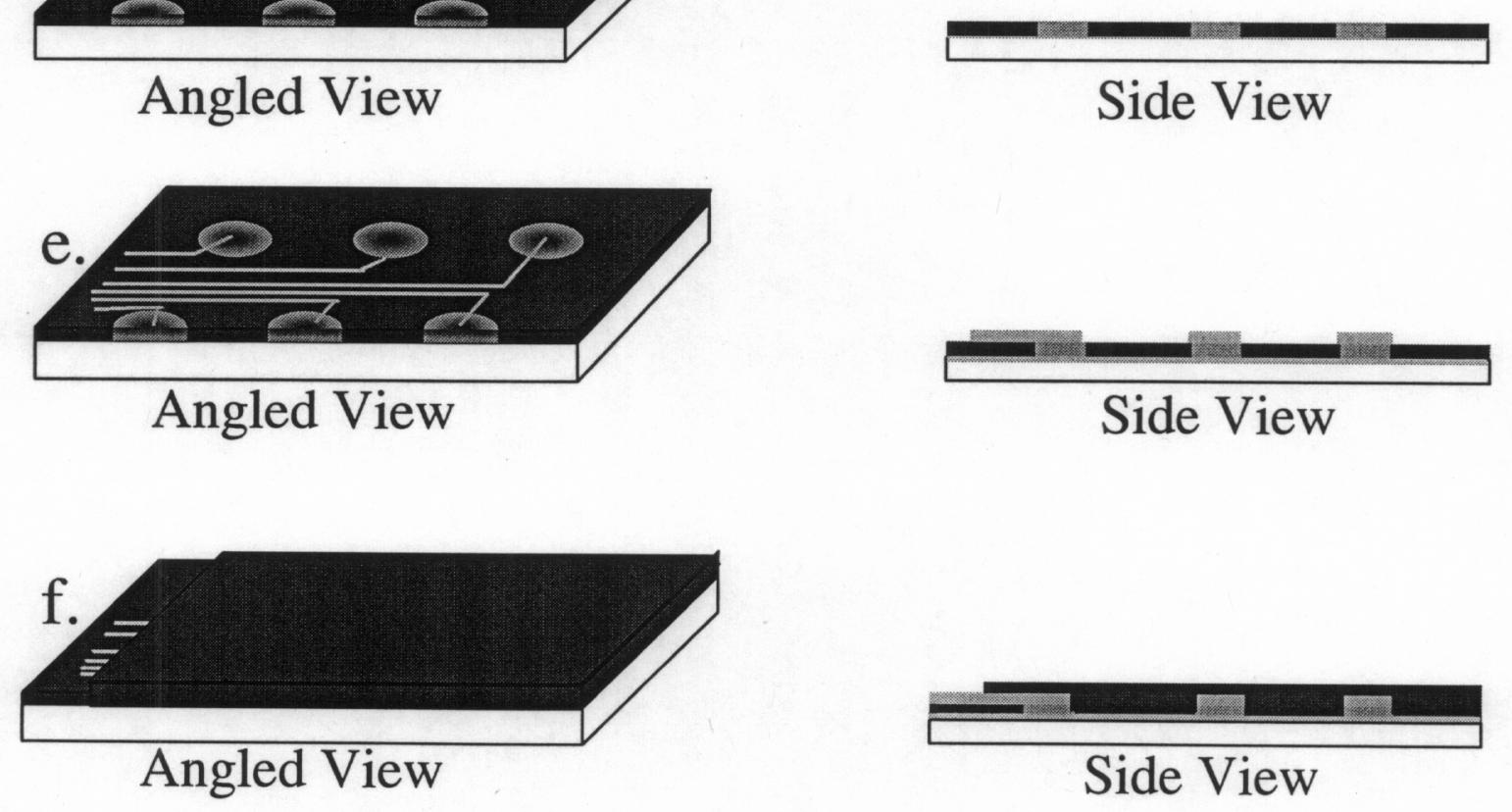


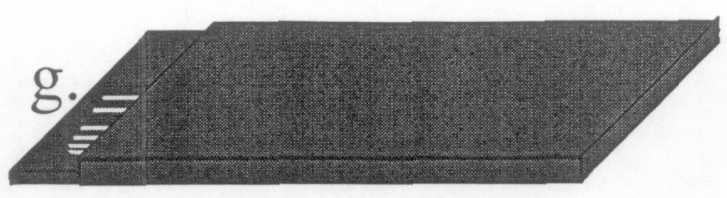

Angled View

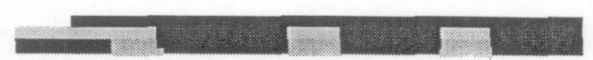

Side View

Figure 4.2. Illustrations of the $1^{\text {st }}$ generation fabrication design plan a) Thick photoresist features are patterned on a handle wafer that has been pre-coated with a metal seed layer. b) A PDMS film is spun onto the wafer and cured c) Stripping the photoresist results in a patterned PDMS film with holes through to the underlying seed layer. d) Platinum is then electroplated through the PDMS to form an array of electrical stimulators e) metal traces are deposited onto the PDMS to serve as an electrical interface to the microelectrode array f) Selectively encapsulating the microelectrode array and the leads with a second PDMS layer for electrical passivation g) device removed from the wafer

\subsection{FABRICATION}

\subsubsection{Prototype-1 Electrode Development}

The fabrication process began with a four-inch gold-coated silicon wafer. It was found to be necessary to leave $2 \mathrm{~mm}$ ring along the edge of the silicon wafer uncoated with gold to prevent the PDMS film from lifting during processing. A sacrificial photoresist layer was used to create the holes in the PDMS film for electroplating. Photoresist (AZ ${ }^{\circledR}$ PLP 100XT, Clariant) was spun onto the handle wafer and baked at $60^{\circ} \mathrm{C}$ for $20 \mathrm{~min}$. The photoresist was then UV exposed using a high-resolution transparency as the photomask (Imagesetters, Pleasanton CA). The pattern was developed (AZ 400K: $\mathrm{H}_{2} \mathrm{O}$ 1:3 ratio) and PDMS was applied at a spin rate of $2500 \mathrm{rpm}$ for $20 \mathrm{sec}$. As seen in the SEM pictures in Fig. 4.7c, the PDMS covered the tops of the photoresist features making it difficult to remove the photoresist and clear the holes for electroplating. This was minimized through characterization of appropriate spin speed and time needed to minimize streaking and by allowing the PDMS to planarize at room temperature before curing at an elevated temperature. Planarization was observed to be 
completed by visually inspecting the disappearance of any streaks formed near patterned features following the spinning process. Ninety seconds was determined to be the necessary spin time to minimize the streaking effect. After spinning the PDMS it is cured at $66^{\circ} \mathrm{C}$ for one hour. Once cured, the photoresist features are gently swabbed to remove excess PDMS caps on top of the photoresist features before stripping the resist. This ensures the removal of the photoresist and the complete clearance of the patterned area. Platinum was then electroplated (Platanex ${ }^{\circledR} \mathrm{III}$ by Enthone) in the PDMS patterned holes. All the electroplating solution manufacturer's recommendations were implemented for the electroplating process.

\subsubsection{Prototype-2 Soak Test and Prototype-3 Implantation}

The handle wafer for soak test prototype has no gold coating, while the handle wafer for the implantation prototype is gold coated. In order to deposit metal traces on PDMS, first photoresist must be patterned on to the PDMS for the lift-off process. Since PDMS is a soft elastomeric substrate, the photoresist manufacturer's recommendation for spin speeds, bake time, exposure, and developing time did not apply. All these parameters had to be characterized for patterning photoresist onto PDMS surface. PDMS is spun at $2500 \mathrm{rpm}$ for $20 \mathrm{sec}$ and cured for 1 hour at $66^{\circ} \mathrm{C}$. Once the PDMS film is cured, photoresist (AZ ${ }^{\circledR} \mathrm{PLP} 100 \mathrm{XT}$, Clariant) is spun onto the film surface and baked. Prior to photoresist application, the wafer is placed in an oxygen plasma to oxidize the surface. The photoresist features are then exposed and developed as previously described and placed for a second time in the oxygen plasma to activate the newly exposed PDMS surface, and promote adhesion of the metal. A $150 \mathrm{~nm}$ gold film 
is then e-beam evaporated onto the wafer using $20 \mathrm{~nm}$ of titanium as the adhesion layer without breaking vacuum. The metal adheres to the exposed PDMS regions and the excess metal is removed through a lift-off process by placing the wafer in acetone and then rinsing with isopropanol (IPA). The wafer is then prepared for passivation by rinsing with ethanol and drying gently. An oxygen plasma treatment is again performed before passivation to ensure adhesion of the second PDMS layer. An acetate stencil-like mask is then placed on the metal traces, and the second PDMS layer is spun onto the substrate. The stencil is removed prior to curing at $66^{\circ} \mathrm{C}$ for one hour, exposing regions of the traces necessary for electrical connections. After the PDMS is cured the wafer is diced to produce separate devices.

\subsection{EXPERIMENTAL APPROACH}

A number of experiments are used to evaluate the viability of different aspects of the process. These evaluation experiments are performed in conjunction with our collaborators at University of Southern California (USC), $2^{\text {nd }}$ Sight LCC, and Oak Ridge National Laboratory (ORNL).

\subsubsection{PDMS Thickness Characterization}

The PDMS used for this work is Sylgard ${ }^{\circledR} 184$ from Dow Corning corporation. It is mixed in a 10:1 mass ratio of resin and curing agent respectively and degassed to remove all air bubbles. The appropriate thickness of PDMS that would allow for easy handling and implantation is determined in conjunction with our collaborators at USC. Seven 
wafers with PDMS thicknesses varying from approximately 3 to $70 \mu \mathrm{m}$ are sent to USC for evaluation of handling and surgical manipulation. Table 4.1 and Plot 4.1 are obtained by measuring resulting PDMS film thickness following a series of spin rates at two spin times, 20 and 90 seconds. Using an exponential fit of the data, Eqns. 4.1 and 4.2 were generated relating film thickness to spin speed. These equations provide a means to approximate spin speeds needed to achieve desired PDMS film thickness.

Time $=20 \mathrm{sec}$

$$
\begin{array}{ll}
\text { Time }=20 \mathrm{sec} & \text { speed }(\text { rpm })=(\mathbf{L N}(\text { thickness })-\mathbf{L N}(121.09)) /(-0.0006) \\
\text { Time }=90 \mathrm{sec} & \text { speed }(r p m)=(\mathbf{L N} \text { (thickness })-\mathbf{L N}(53.349)) /(-0.0005)
\end{array}
$$

Table 4.1. PDMS thicknesses

\begin{tabular}{|c|c|c|}
\hline & at 20 sec & at 90 sec \\
\hline Speed (rpm) & thickness (microns) & thickness (microns) \\
\hline 7000 & 2.6 & 1.5 \\
\hline 6000 & 4 & 2.5 \\
\hline 5000 & 7 & 3.5 \\
\hline 4000 & 12 & 4.6 \\
\hline 3000 & 23 & 8.2 \\
\hline 2000 & 40 & 20 \\
\hline 1000 & 71 & 45 \\
\hline
\end{tabular}

\section{PDMS Spin Graph}

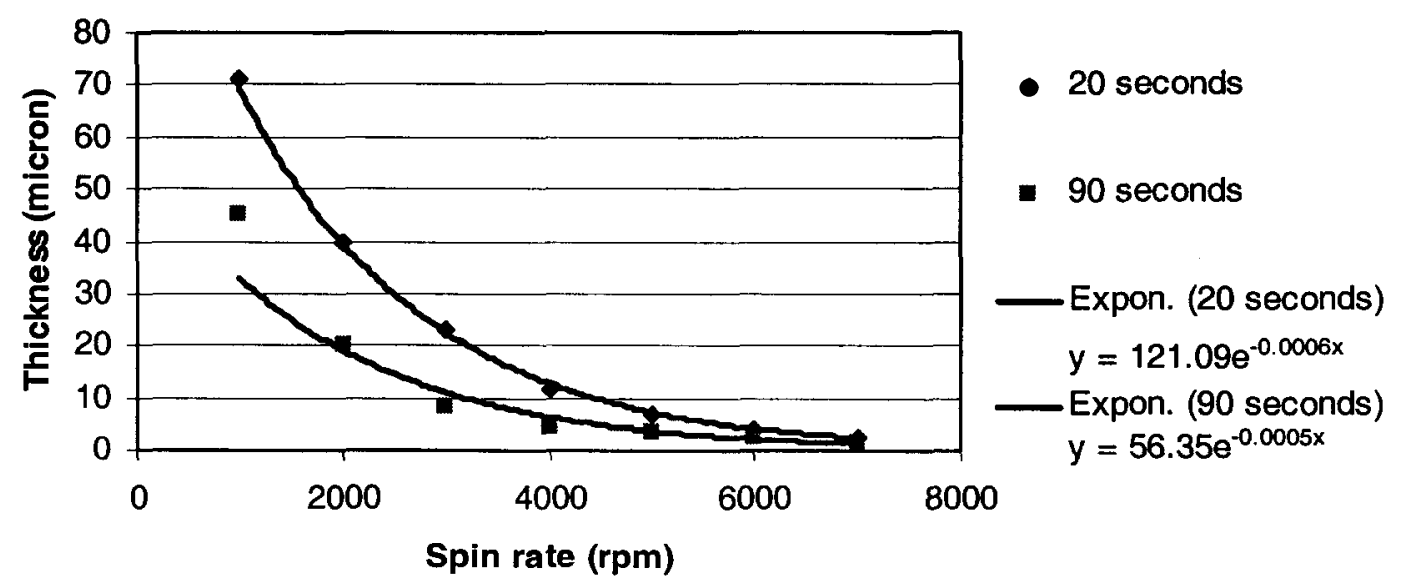

Plot 4.1. PDMS thickness as a function of spin rate at 20 and 90 seconds with exponential trend lines 


\subsubsection{Adhesion Tape Test}

The Tape test is the method used to ensure complete metal adhesion to the PDMS. Although there is no easy way to quantify interfacial atomic-bond strengths between the metal and the PDMS, we have used the tape test to qualitatively measure metal adhesion to the PDMS surface. Schematically illustrated in Fig. 4.3a, the test can distinguish between complete lifting, partial lifting or complete adhesion. The adhesive tape test is performed on each fabricated wafer in several locations to ensure metal adhesion. Scotch $^{\circledR}$ Tape is applied to the metalized PDMS and pressed down firmly to ensure complete contact between the tape and the metal. The tape is consistently pulled back at approximately $180^{\circ}$ angle at $5 \mathrm{~mm} / \mathrm{sec}$, hence not to rip the PDMS. The tape is visually checked for any metal presence, Fig. $4.3 \mathrm{~b}$, and the metal trace is checked for electrical continuity. This experiment is the initial indicator of whether or not the metalization process was successful. Complete adhesion is needed for functional devices; therefore, useful wafers have no metal lifting onto the tape. For this experiment 5 wafers were fabricated and tested. 
a.

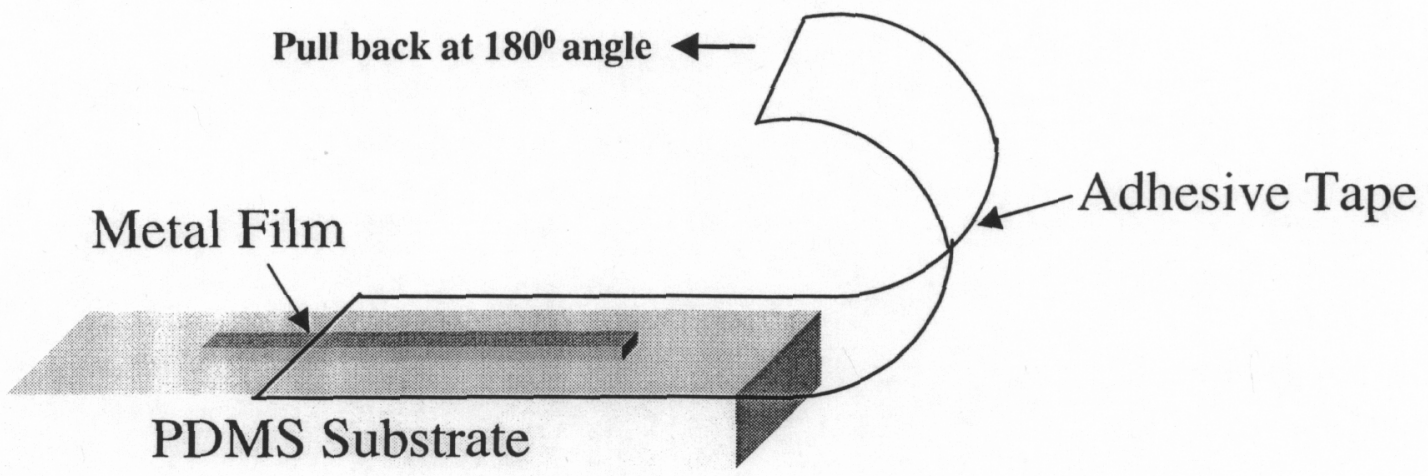

b.

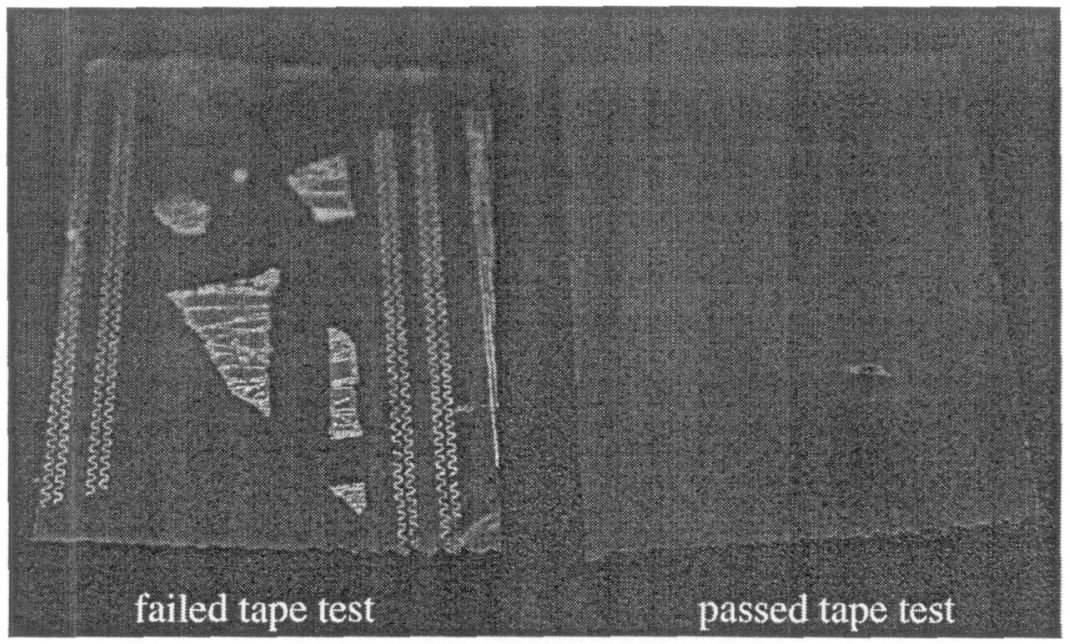

Figure 4.3. a) Adhesion tape test b) tape test failed tape test vs. passed tape test

\subsubsection{Electrical Continuity}

The $1^{\text {st }}$ generation devices are primarily to conduct fabrication feasibility tests, soak tests, and acute implant tests; therefore, a means of transmitting signals and monitoring the condition of the metal traces is necessary. The electrical continuity of each device is tested using an ohm-meter to determine if the device is functioning. This test is conducted before and after passivation. For the $1^{\text {st }}$ generation devices the metal traces formed two loops along the length of the test structure with contact pads at either end of the trace serving as probe points as shown in Fig. 4.4. These traces were $50 \mu \mathrm{m}$ wide lines that terminated at $1 \mathrm{~mm} \mathrm{X} 0.5 \mathrm{~mm}$ contact pads. The contact pads are gently 
probed to ensure minimal damage onto the metal traces. If there is a break or if a break develops during handling in the metal traces, then this would be easily observable through direct resistance measurement of the metal trace. The same wafers that were used for the adhesion tape test were used for measuring electrical continuity. Each of the 5 wafers contained 26 devices for a total of 130 devices tested.

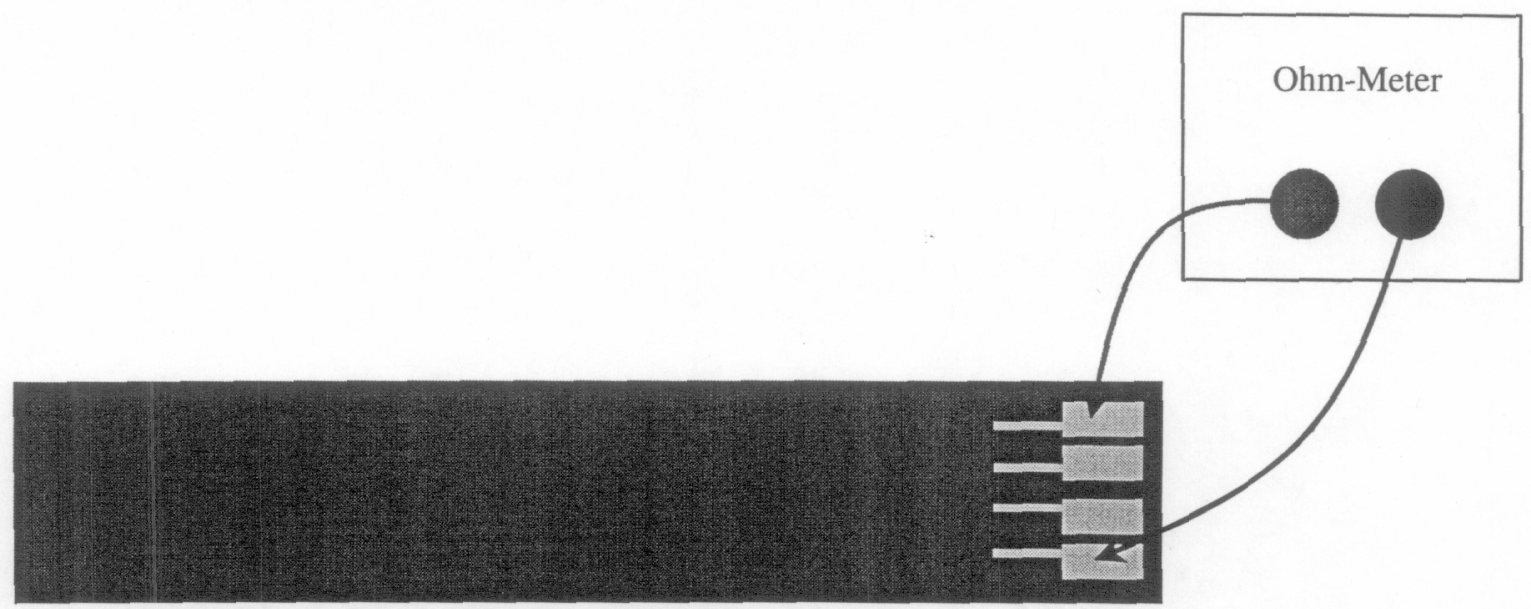

Figure 4.4. Electrical continuity testing

\subsubsection{Soak Test Experiments}

Soak tests are conducted to determine if the passivation layer is functioning properly by insulating the metal traces and preventing leakage, which can corrode and short the metal traces. Also, the soak tests are to examine if the PDMS substrate can sustain the harsh environment of the eye and potentially survive up to 100 years. Accelerated soak testing of metalized PDMS passivated samples are conducted in phosphate buffer solution (PBS) at a temperature of $87^{\circ} \mathrm{C}$ at $2^{\text {nd }}$ Sight LCC. The samples for the soak tests consisted of metal traces sandwiched between two PDMS layers on a bare silicon wafer as shown in Fig. 4.5. The $80 \mathrm{~mm}$ long device has 4 metal resistors patterned on top of a layer of PDMS. A second layer of PDMS selectively passivates the 
metal traces, leaving the end connectors exposed. Silicon is known to corrode in saline, however it is merely serving as a means for handling the silicone devices during testing.

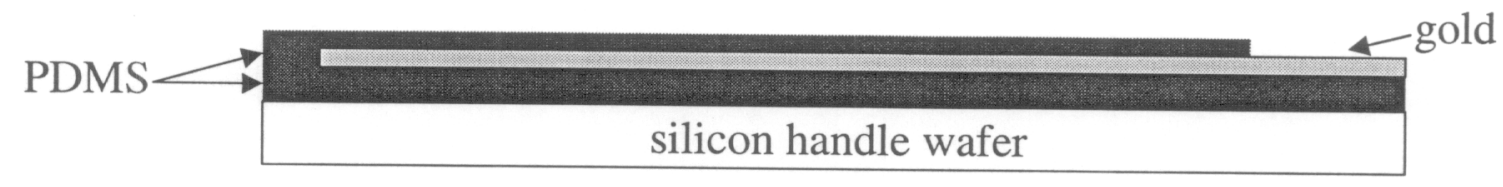

Side View
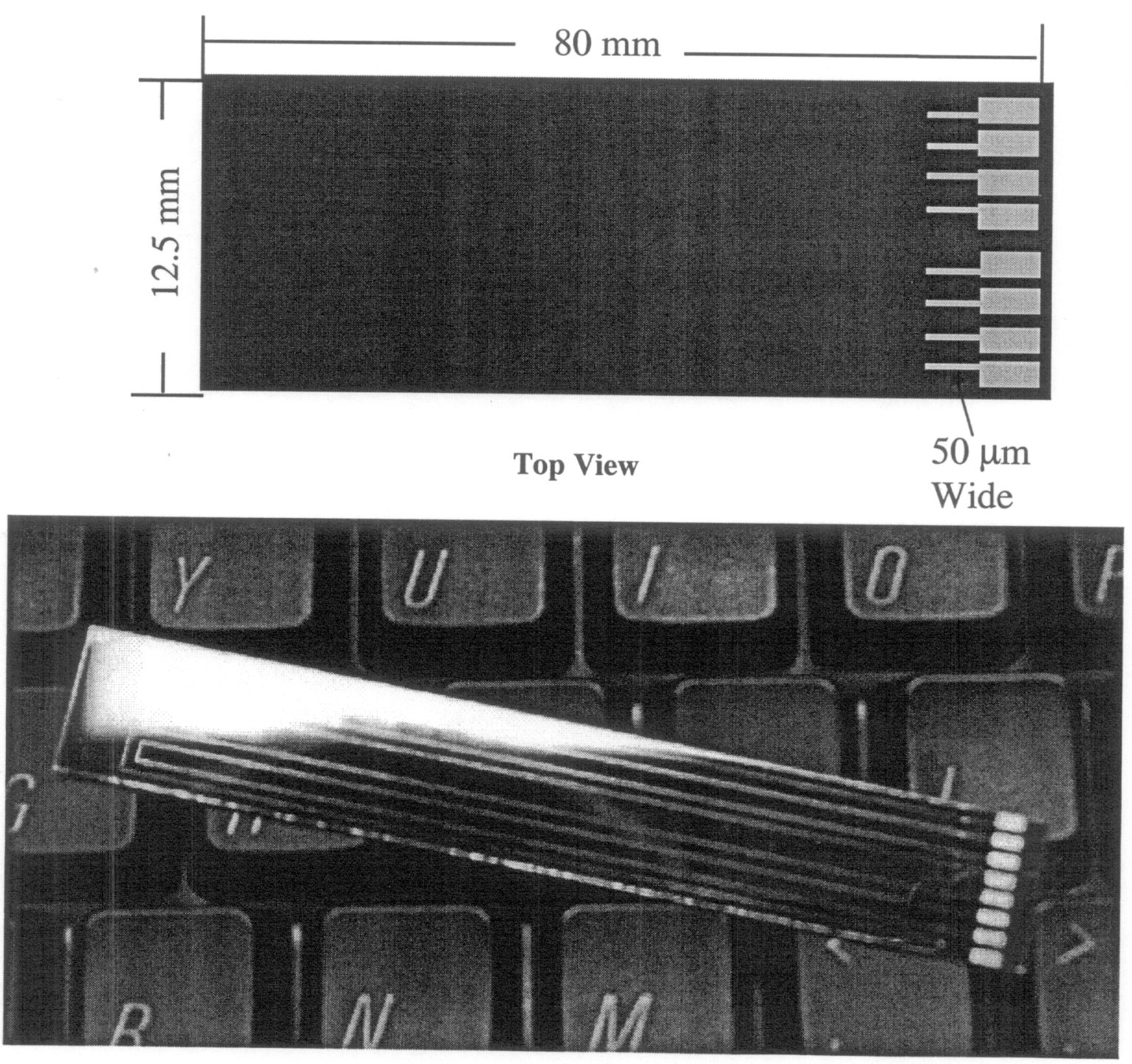

Figure 4.5. PDMS soak test prototype 


\subsubsection{Acute Surgical Implantation}

Initial implantation evaluation tests are conducted in collaboration with USC to determine: 1) ease of handling of devices by surgeons, 2) if the PDMS device can be inserted into the eye without tearing or damaging the metal traces, 3) if the metalized PDMS conform to the curvature of the eye. The PDMS thickness evaluation along with restrictions set by the surgeons guided in determining the dimensions for the $1^{\text {st }}$ generation implant prototype. The dimensions are set to be $3 \mathrm{~mm}$ wide by $40 \mathrm{~mm}$ long by $50 \mu \mathrm{m}$ thick. The width of the device, $3 \mathrm{~mm}$, is constrained by the size of incision that can be made into the eye to insert the device. The length is set at $40 \mathrm{~mm}$ to allow the device to extend outside the eye for diagnostic probing during the implantation, Fig. 4.6. This prototype consists of two layers of PDMS sandwiching two metal traces. The first layer of PDMS has the metal traces deposited on it as described in previously and the second layer is a passivation layer over the metal traces. This is a free-standing device, meaning after fabrication the device is removed from the handle wafer. The implantation procedure is performed in a canine eye, which is approximately the size of a human eye.

Approximately one-third of the prototype device is inserted into the canine eye as seen in Fig. 4.8. All procedures were approved by the IACUC (Institute of Animal Care Use Committee) of the University of Southern California. Testing of surgical applicability and contact with the curvature of the retina was performed. The integrity of the thin film traces onto the PDMS were electrically tested before and after the implantation. Drs. Fujii and Guven implanted the epiretinal device into the eye of one dog, following standard 3-port vitrectomy procedure. The procedure begins with detaching the musculature around the eye ball and suturing three strings for controlling 
the eye. The pupil is dilated using phenylephrine $2.5 \%$ with a magnifying lens placed on top. The magnifying lens, in combination with the surgical microscope aids the surgeon in seeing clearly in-side the eye during the procedure. An incision is made on the side of the of the eye through the sclera, through which the vitreous is suctioned out. In the mean while a tube is attached to the eye to provide a port for saline to be pumped through continuously to maintain ocular pressure. After the vitreous is removed, the device is threaded through. A second incision is made on the opposite side of the eye through the sclera through which forceps were used to grasp the deice and help pull it through and position it on the retina. Since this is an acute implantation procedure, the device is removed prior to suturing up the eye.

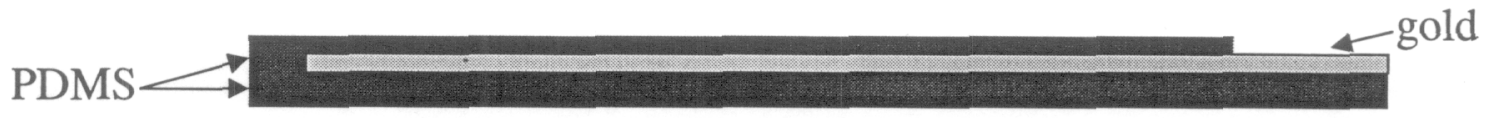

Side View

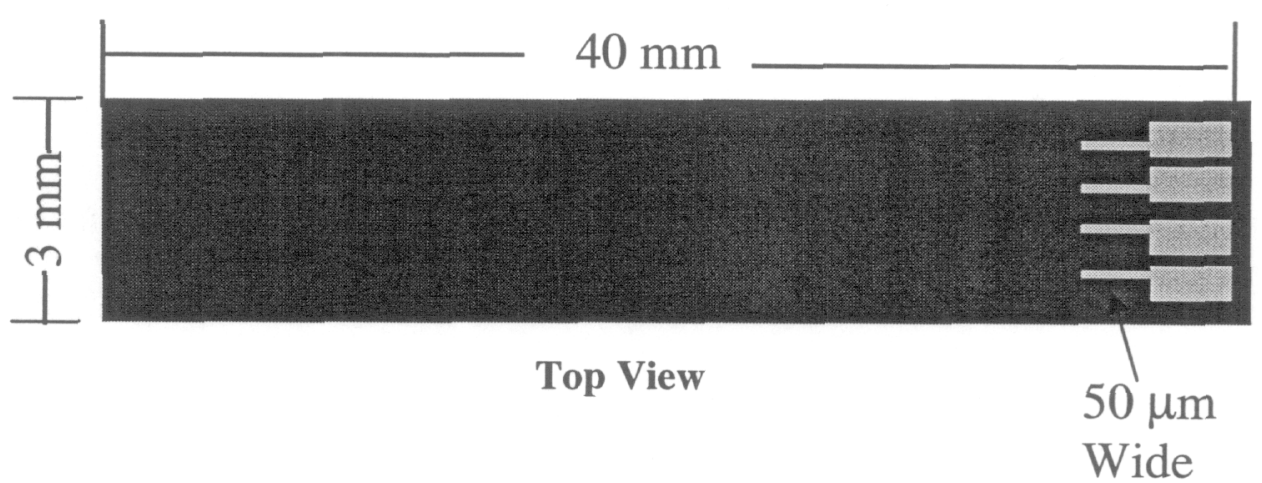




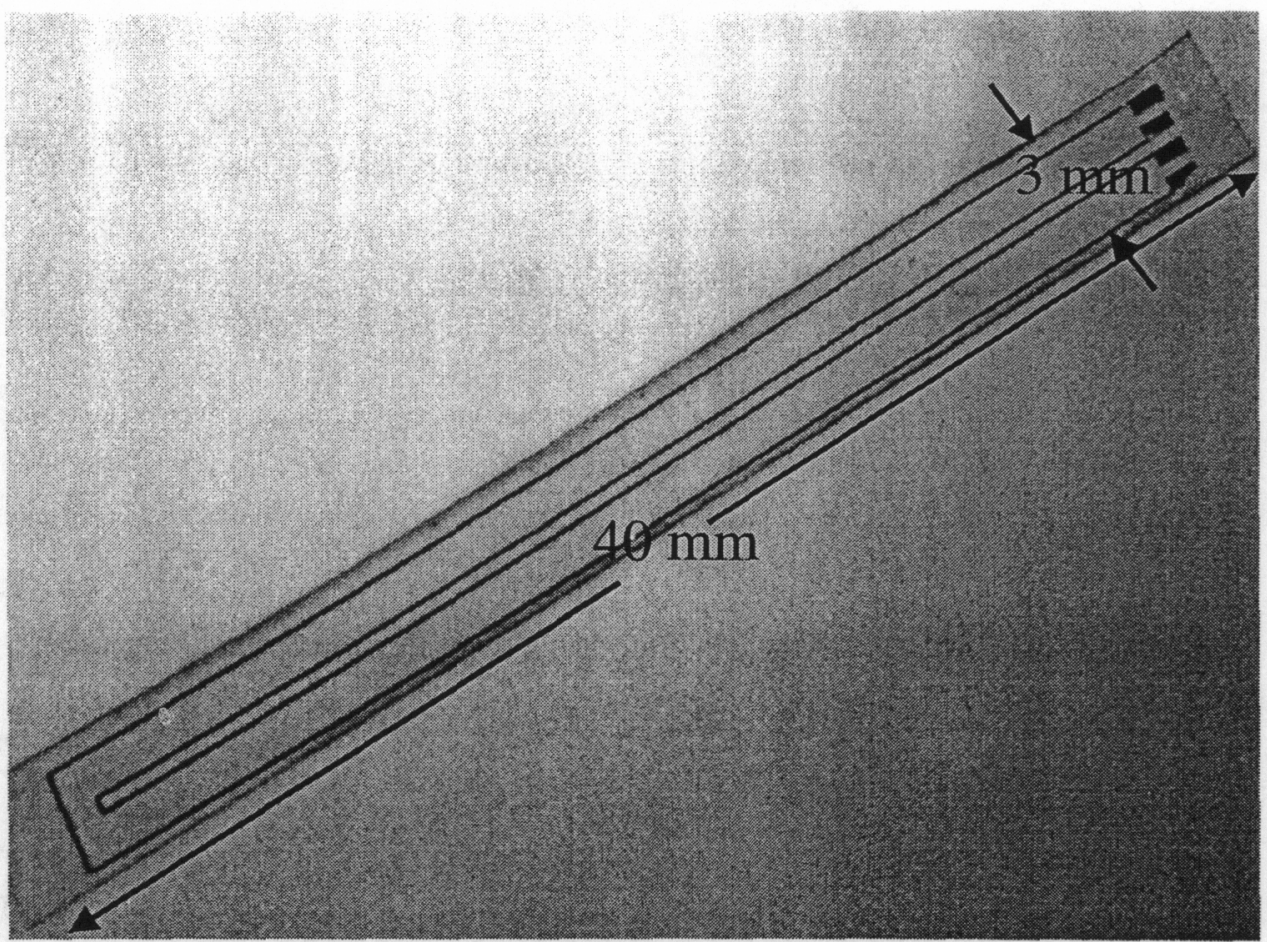

Figure 4.6. Evaluation prototype for surgical implantation

\subsection{RESULTS}

\subsubsection{Fabrication Results}

Modifications were constantly adopted as needed during the fabrication process development. Figure 4.7 presents the fabrication results. The SEM images in Fig. 4.7c confirm that gently swabbing the tops of the photoresist features ensures removal of PDMS cap and photoresist. In the SEM images in Fig. 4.7d, there appears to be problems associated with residual stresses causing the electroplating process to be unsuccessful.

The $1^{\text {st }}$ generation soak test and surgical implantation prototypes did not have platinum electrodes. They consist of metal traces sandwiched between two PDMS layers. The optical image in Fig. 4.7e indicates a wrinkling effect taking place due to residual stresses in the metal and PDMS film. Spinning the PDMS for the selective passivation seems to stress the metal leads and cause them to break as seen in Fig. 4.7f. 


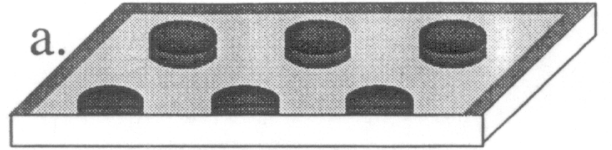

Angled View

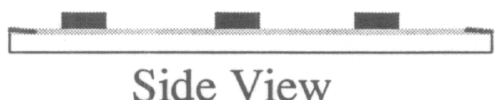

Side View

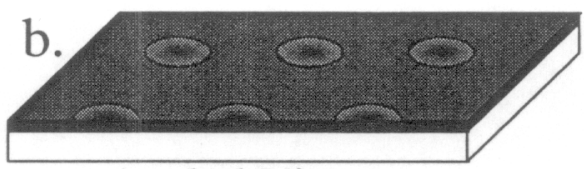

Angled View

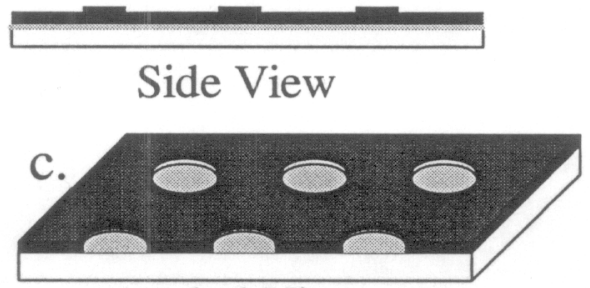

Angled View
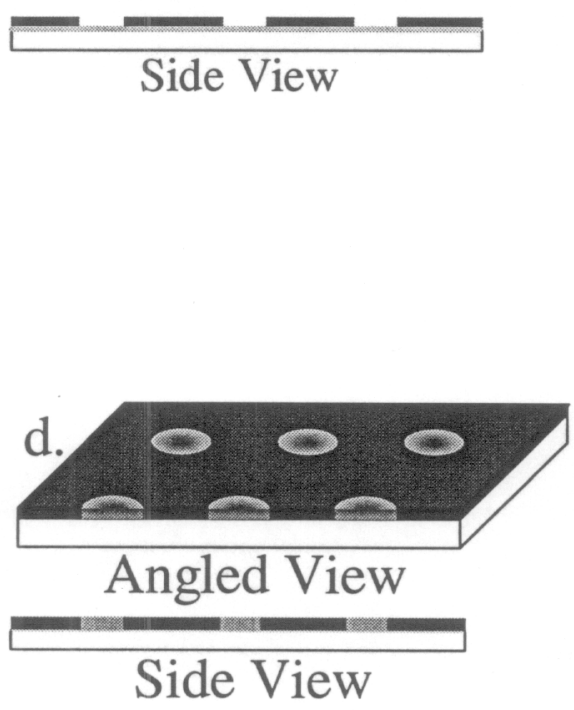
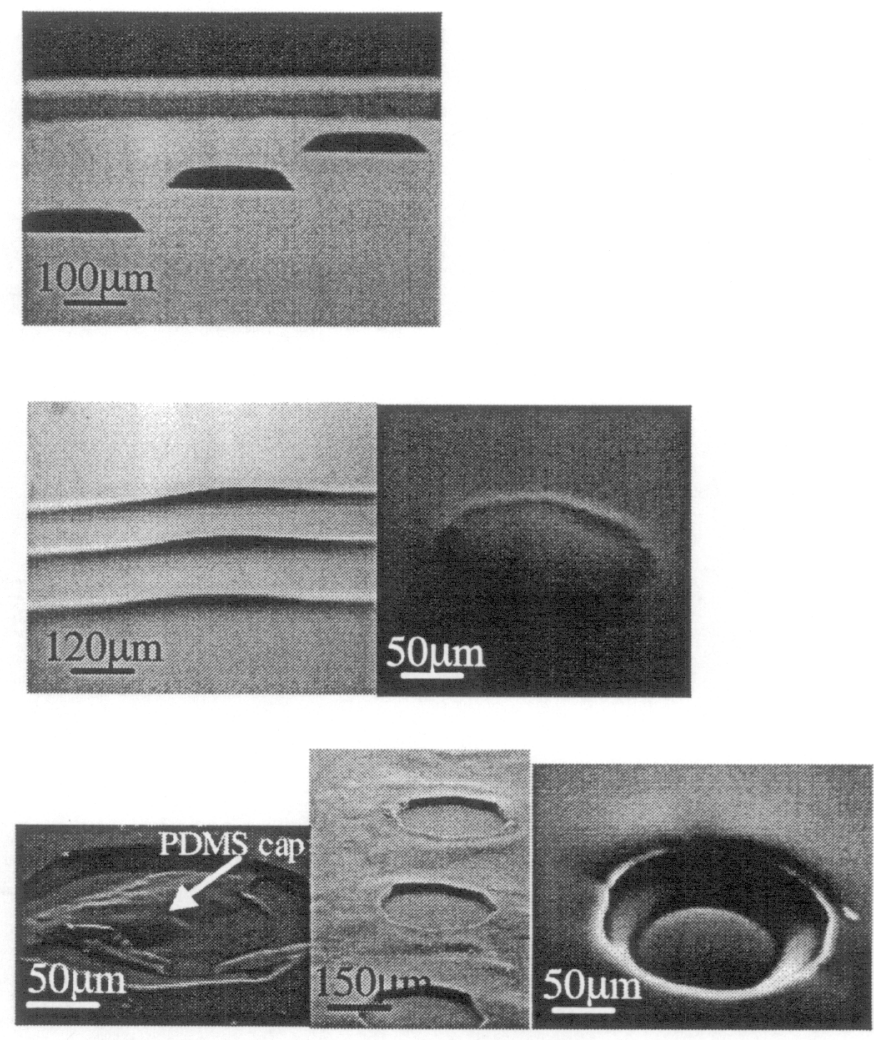

Unswabbed

Swabbed
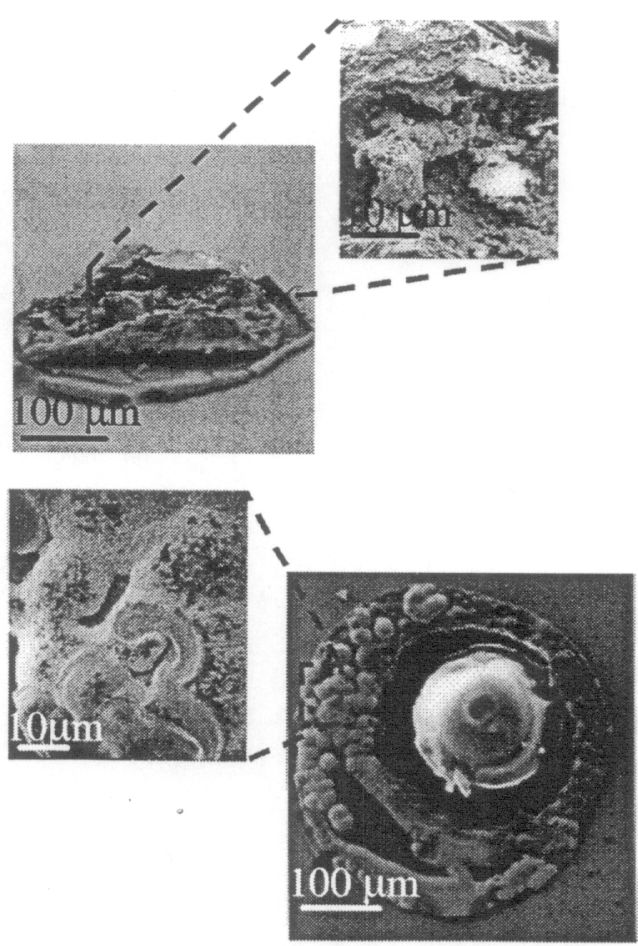

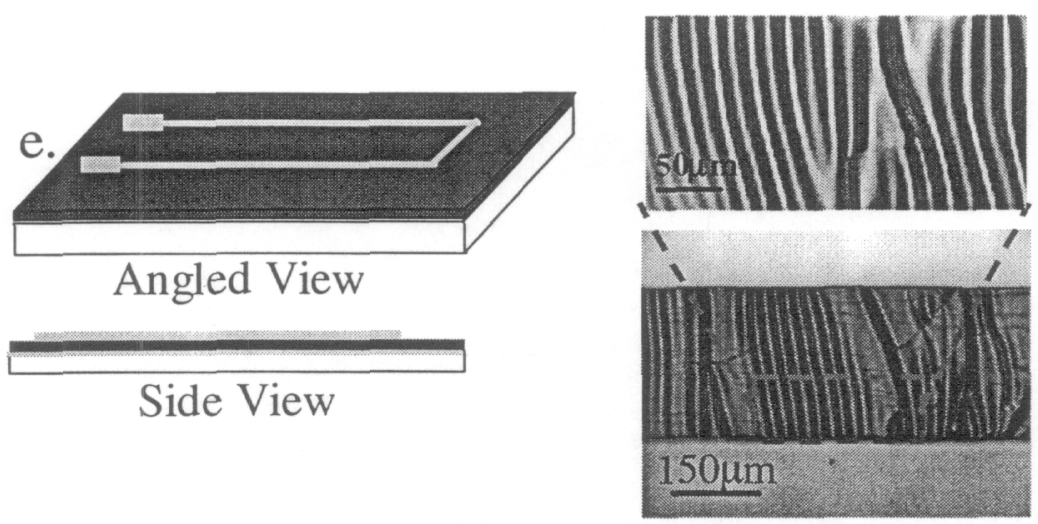

break in metal lead due to passivation

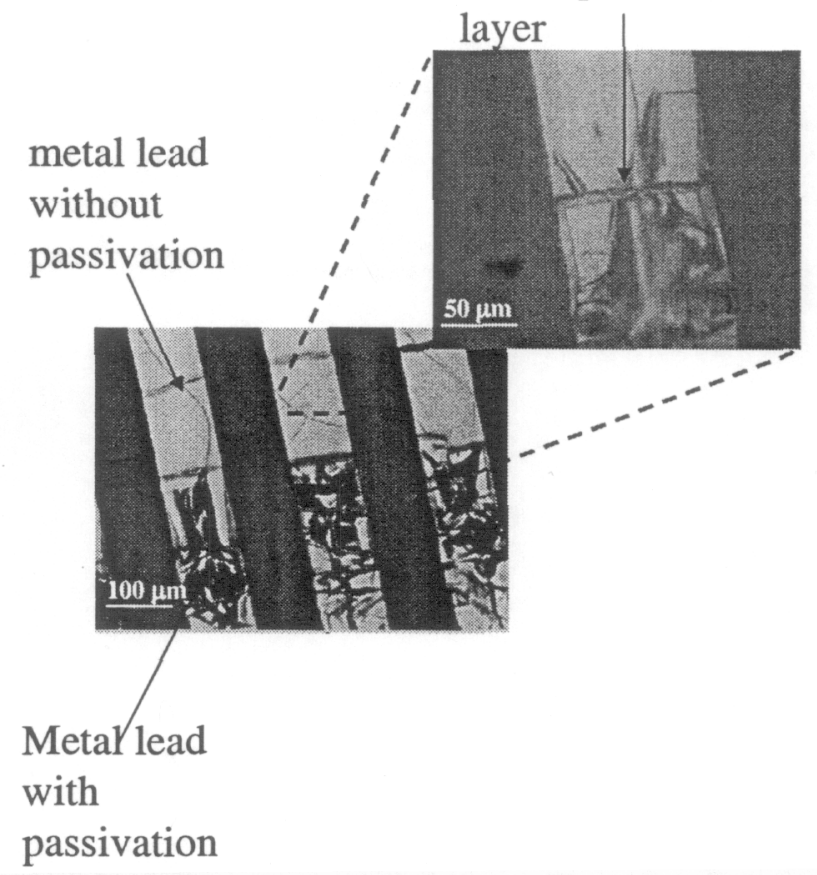

Figure 4.7. $1^{\text {st }}$ generation fabrication process flow along with the corresponding SEM and optical images of the features formed following each fabrication step on the right a)Thick photoresist (AZ ${ }^{\circledR}$ PLP 100XT, Clariant) was spun and patterned onto a gold coated wafer to a thickness of $30 \mu \mathrm{m}$, as shown in the SEM picture on the right b) PDMS was spun over the patterned photoresist features and cured c) Once cured, the photoresist features are gently swabbed to remove excess PDMS caps on top of the photoresist features before stripping the resist d) Platinum was electroplated in the patterned PDMS holes using Platanex ${ }^{\circledR}$ III from Enthone e) Metal traces were patterned on the PDMS surface f) PDMS passivation layer was spun on top of metal traces using an acetate stencil-like mask 


\subsubsection{PDMS Thickness Characterization Results}

After evaluating the various free-standing PDMS thicknesses, Humayun's group determined that $50 \mu \mathrm{m}$ was thick enough to handle, but thin enough to conform to the retina. PDMS films less than $50 \mu \mathrm{m}$ tended to easily shrivel up onto itself, making them very difficult to handle. Therefore it was decided that the $1^{\text {st }}$ generation PDMS films would have a total thickness of $50 \mu \mathrm{m}$; with the initial film and the passivation film, being 25 microns each.

\subsubsection{Adhesion Test and Electrical Continuity Results}

Table 4.2. Adhesion and electrical continuity results

\begin{tabular}{|l|l|l|}
\hline & Tape Test / wafer & Electrical continuity/ device \\
\hline Unpassivated & $60 \%$ Passed & $66 \%$ passed \\
\hline Passivated & NA & $21 \%$ passed \\
\hline
\end{tabular}

Sixty percent of the metalized PDMS wafers passed the tape test and $66 \%$ of the devices tested were electrically continuous. However only $21 \%$ of the passivated device remained electrically continuous.

\subsubsection{Soak Test Results}

Following soak testing in PBS at $87^{\circ} \mathrm{C}$ with $5 \mathrm{~V}$ bias, color changes around defect spots was observed on some of the gold traces. The backside of the un-insulated silicon substrate began dissolving during soak in PBS, but this dissolution did not appear to affect the adhesion of PDMS to the silicon.

\subsubsection{Acute Surgical Implantation Results}


As the array was compliant and stretchable, it was placed on the retina smoothly, appearing to fit the curvature of the retina. Device handling during implantation was found to be somewhat difficult outside the eye, due to the tendency of the thin $(50 \mu \mathrm{m})$ devices to curl and the fact the device is relatively long and thin. But once it was in the fluid environment of the eye, handling and manipulation became easier. The array conformed to the retina without causing any apparent stress on the eye. The metal trace near the edge of the devices, where the forceps grasped them during implantation, were found to be damaged during the procedure.

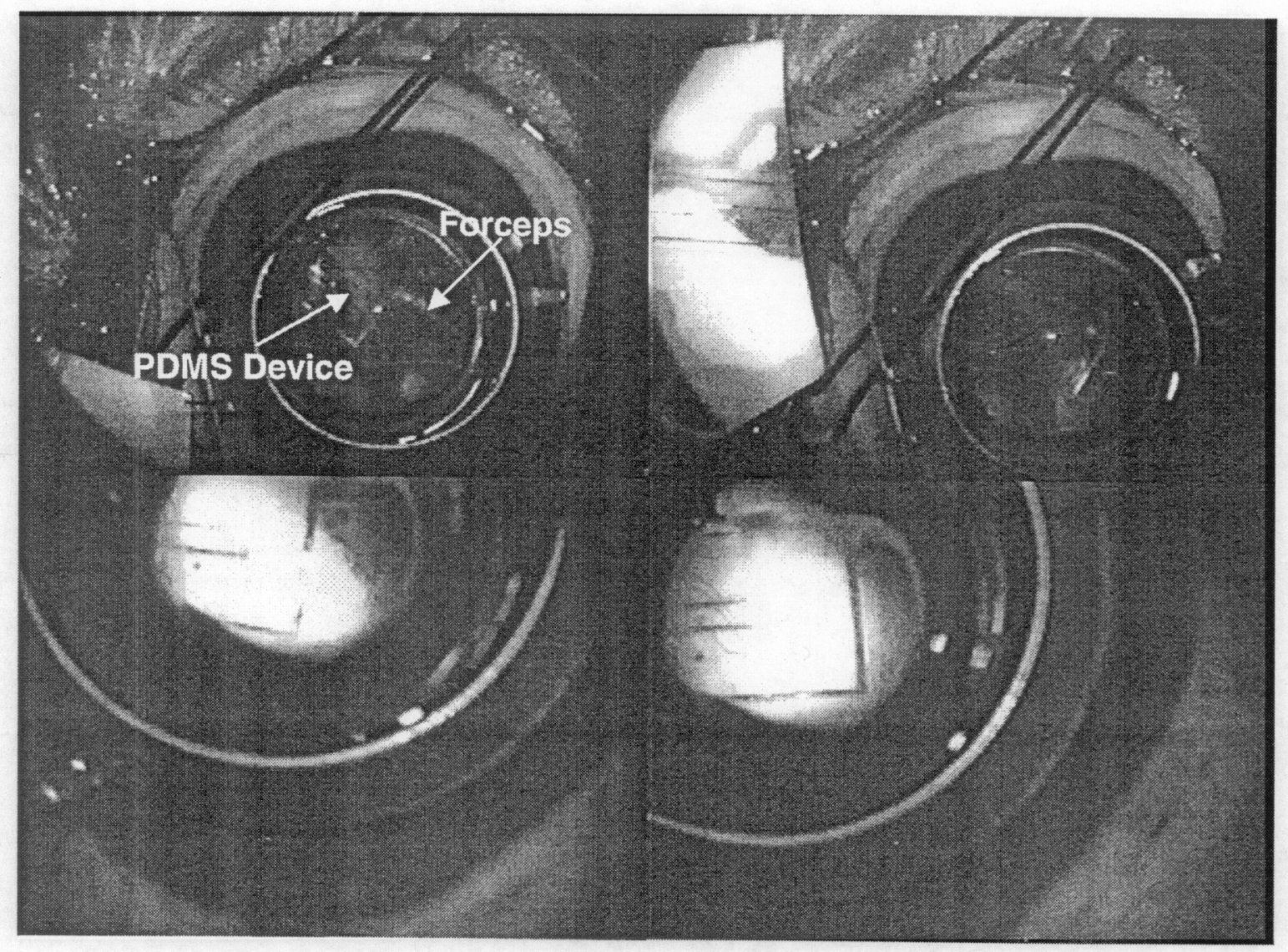

Figure 4.8. PDMS test structure implanted in the eye of a live dog. All procedures were approved by the IACUC (Institute of Animal Care Use Committee) of the University of Southern California. 


\subsection{DISCUSSION}

All of the $1^{\text {st }}$ generation results were very promising and led to valuable insights into the $2^{\text {nd }}$ generation device design (see chapter 5). Fabrication difficulties encountered during the process prompted a number of modifications, such as leaving areas on the silicon wafer uncoated with gold to prevent the PDMS film from lifting during processing. Others include allowing the PDMS to planarize before cuing to minimize streaking and gently swabbing the PDMS surface to ensure complete photoresist removal.

A valuable lesson learned through the fabrication process development is that understanding the issues relating to surface properties is of vital importance to the development of reliable PDMS fabrication technology. Difficulties encountered with inconsistent metal adhesion and electroplating resulted in the closer examination of surface properties of PDMS following oxygen plasma treatment and curing times (see chapter 6). Electroplating platinum was a major hurdle due to non-uniform and highly stressed films. Platinum electroplating is an extremely delicate and sensitive process and is easily affected by any variations in parameters or any contaminates present on the surface of the substrate being plated. There are many parameters that influence the outcome of the electroplating. Amongst them are current density, concentration of the electrolytes, temperature, agitation, impurities, anode to cathode ratio and distance, and $\mathrm{pH}$. Optimizing all of the parameters is vital to achieve low stress high quality films. Although it is known that the intrinsic residual stress in platinum makes it a challenging material for electroplating [1], in this case surface contamination was found to be the primary obstacle, as discussed in chapter 6 . The surface analysis discussed in chapter 6 
revealed that PDMS surface contamination is very difficult to eliminate, therefore making electroplating problematic.

The soak test results for the $1^{\text {st }}$ generation devices look to be very promising with a few defect spots caused by pinholes in the PDMS passivaiton layer. These pin holes are caused by particulates that may of have landed on the PDMS surface during transportation from the clean room to another laboratory where the curing oven resides. Interfacing the polymer microelectrode array to silicon integrated circuit (IC) electronics is thought to be a key challenge, both in terms of making multiple reliable electrical interconnections and sealing the IC from the harsh environment within the eye. The fact the PDMS film did not delaminate form the silicon surface during the soak test is a very promising indication that it is possible to create a reliable bond between silicon chip and PDMS that can withstand the harsh environment of the eye. After bonding the chip can be passivated with a PDMS layer to prevent corrosion from the backside.

Other modifications to the process flow design were introduced following the observation of the acute surgical implantation. Testing the device while it was implanted was challenging and a need for connector became imminent. Both the incorporation of the new modifications and the development of the connector are discussed in the $2^{\text {nd }}$ generation design scheme in the next chapter.

Aside from the stresses induced by external loading, such as handling and surgical implantation, the device also experiences residual stresses. This is evident by the wrinkling of the metal traces on the PDMS surface and by breaks in the traces due to the passivation layer. Two factors contribute to the wrinkling of the metal traces. First, the metal film is deposited in a state of compressive residual stress, causing it to wrinkle 
slightly on the cured PDMS layer. Second, the cured PDMS layer is in a state of tensile residual strain from the fact that it is spun-on and therefore constrained by the rigid handle wafer while it undergoes a volume contraction associated with the curing process. When released from the substrate, the PDMS contracts, enhancing the compressive stress in the metal layer, and contributing to the visible wrinkling of the metal lines. The stresses experienced from depositing metal traces on top of spun and cured PDMS films appear to enhance the stretchabltiy of the traces (see chapter 7). However, the stresses induced by the passivation layer is detrimental to the metal traces. The passivation layer caused breaks in the metal trace as seen in Fig. 4.7f. We speculate that these breaks result from the PDMS spinning process and from the volume contraction associated with the curing process. To eliminate this problem membrane transfer is used for passivation in the $2^{\text {nd }}$ generation devices rather than spinning the PDMS directly onto the metal traces. The issue of mechanical stresses of the metal traces is discussed in greater detail in chapter 7. Examining the residual stresses that evolve during the fabrication process will aid in the optimization of the parameters to obtain robust devices.

\subsection{REFERENCE}

[1] A. Misra and M. Nastasi, "Evolution of tensile residual stress in thin metal films during energetic particle deposition.," Journal of Materials Research, vol. 14, pp. 4466-69, 1999. 


\section{CHAPTER 5: $2^{\mathrm{ND}}$ GENERATION DEVICES}

\subsection{INTRODUCTION}

This chapter presents the design, fabrication, and testing of the $2^{\text {nd }}$ generation microelectrode array. The results obtained from the $1^{\text {st }}$ generation processing and surgical implantation presented in chapter 4 combined with surface analysis results presented in chapter 6 serve as a guide in determining the fabrication modifications necessary to improve device yield and integrity. Amongst the most significant modifications are counter measures to prevent surface contamination and the inclusion of micro-molded ribs around the device perimeter to facilitate handling and reinforce electrical traces near the edges. In addition, a custom connector is designed to enable quick and reliable electrical interfacing with the device. A retinal tack hole also is incorporated to aid in attaching the microelectrode array to the retina.

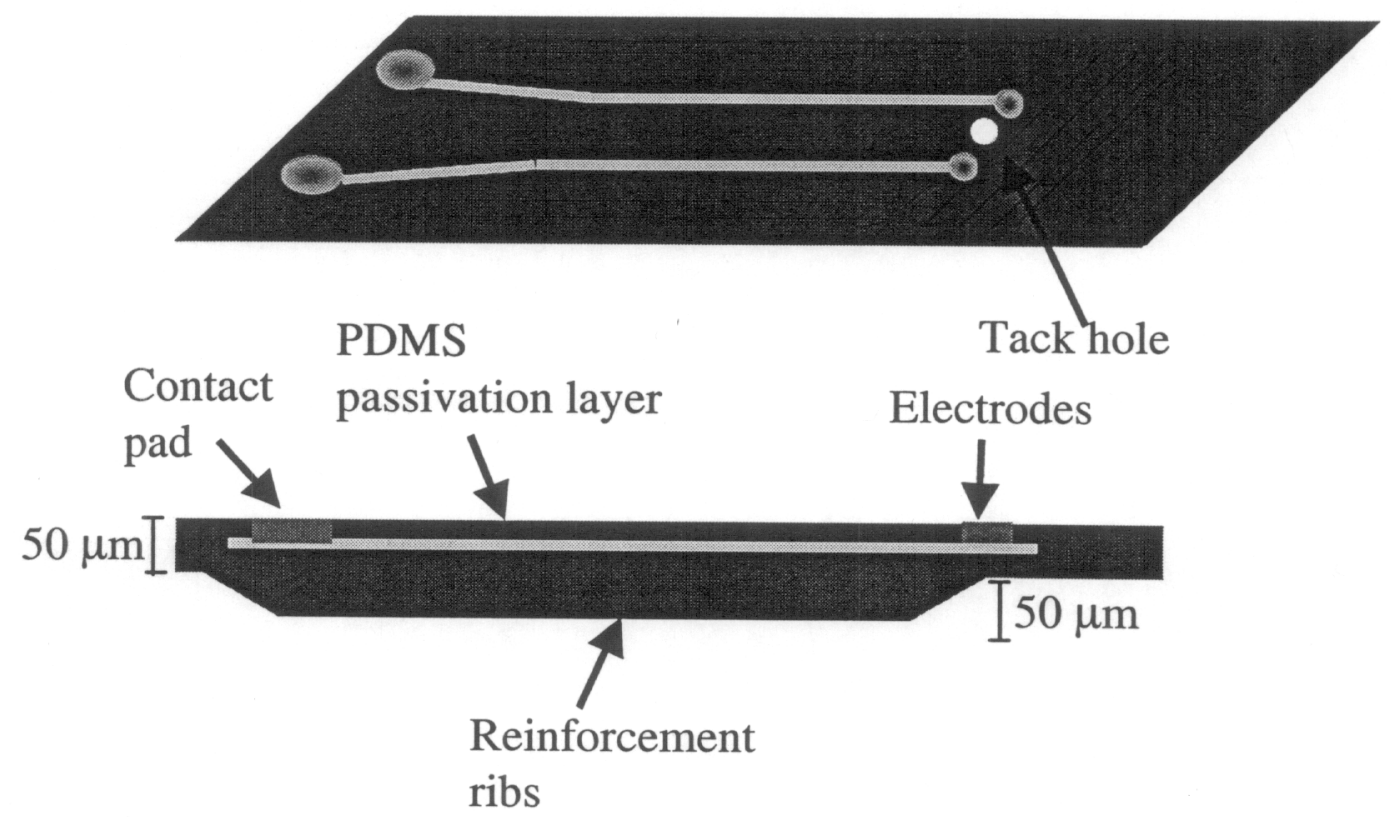

Figure 5.1. Metal traces embedded within two PDMS layers are exposed in the electrode and contact pad regions. 
Similar to the $1^{\text {st }}$ generation concept presented in chapter 4 , the $2^{\text {nd }}$ generation device design has gold metal leads embedded between two thin PDMS films with exposed platinum electrodes, as illustrated in Fig. 5.1. The total thickness of the PDMS film is $50 \mu \mathrm{m}$ with a series of $50 \mu \mathrm{m}$ thick micro-molded ribs along the outer-perimeter of the devices. The length is $40 \mathrm{~mm}$ and width is $4 \mathrm{~mm}$. Arrays of $400 \mu \mathrm{m}$ diameter electrodes are designed in two different configurations. One configuration is referred to as the loop electrode design and the second configuration is referred to as the stimulation electrode design. Both designs are shown in Fig. 5.2. The loop electrode design has $100 \mu \mathrm{m}$ wide continuous metal traces that pass through $400 \mu \mathrm{m}$ electrodes, terminating into contact pads. This design allows for testing of electrical continuity during handling and surgical maneuvering. In the stimulation electrode design each $400 \mu \mathrm{m}$ electrode is connected to an individual contact pad via $100 \mu \mathrm{m}$ wide leads. This design is to be used for ganglion cell stimulation purposes following implantation procedures. 


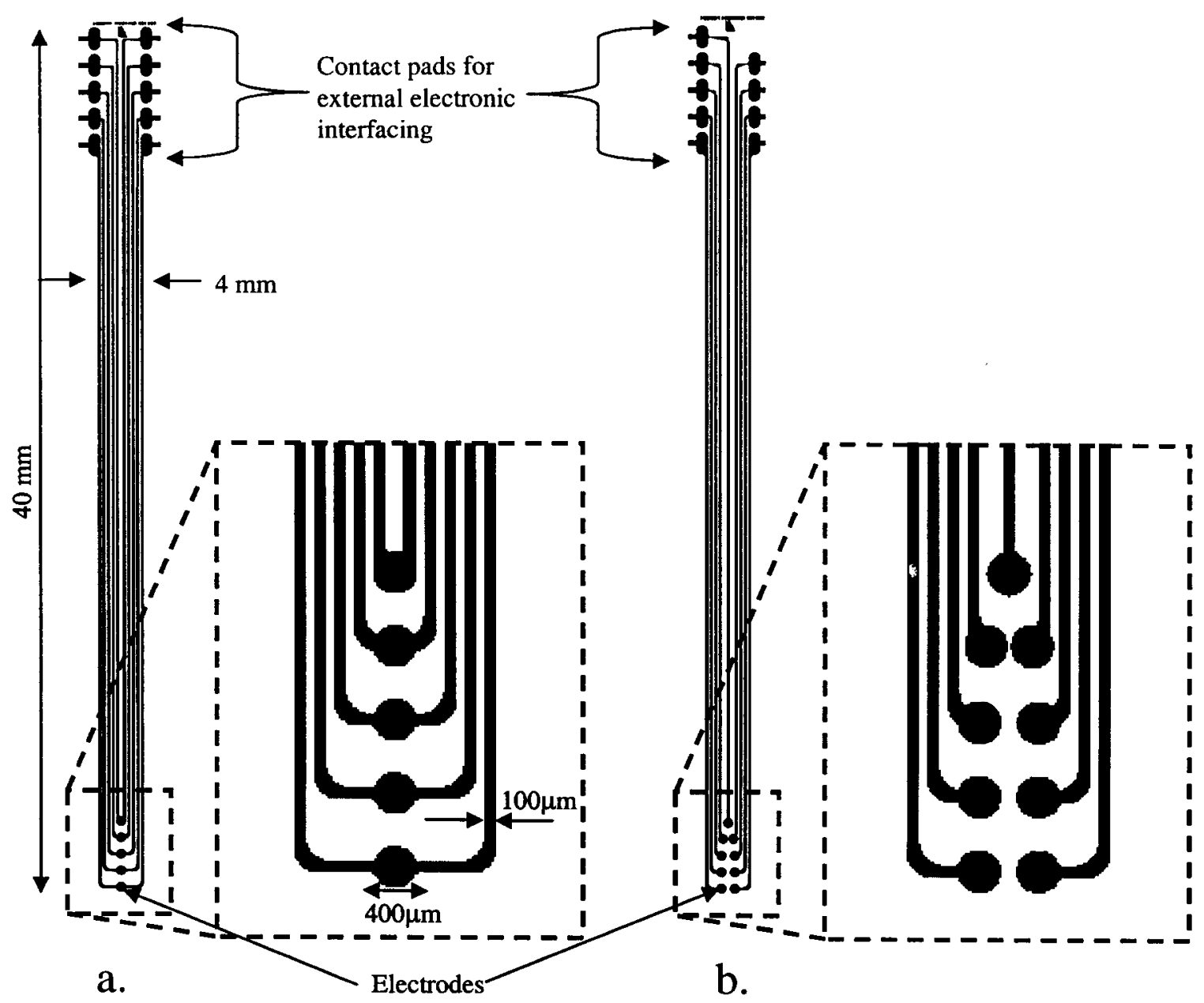

Figure 5.2. a) loop design, b) electrode stimulation design

\subsection{FABRICATION}

\subsection{1 $2^{\text {nd }}$ Generation Fabrication}

The $2^{\text {nd }}$ generation process flow design is illustrated in Fig. 5.3. The complete process flow including complete mask layout is included in Appendix 3. The fabrication process started with anisotropic wet etching [1] of 50-micron deep V-grooves into a silicon handle wafer. These grooves served as the mold for the reinforcement ribs along the boundaries of the microelectrode array devices. After etching, the silicon wafer it 
was then coated with gold as illustrated in Fig. 5.3a. Since PDMS does not adhere to gold, the gold film facilitated removal of the microelectrode array from the wafer after completion of the fabrication process.

Selected regions of the gold on the wafer were etched back to ensure that the PDMS film does not delaminate from the wafer during the fabrication process as illustrated in Fig. 5.3b. PDMS adheres well to the exposed silicon regions. Thick photoresist was spun and patterned on the wafer, as seen in Fig. 5.3c, to form the posts for creating the tack holes for fastening the device to the retina. PDMS was then spun onto the wafer (Fig. 5.3d) at a thickness of $60 \mu \mathrm{m}$ and allowed to planarize at room temperature for $15 \mathrm{~min}$ before curing at $66^{\circ} \mathrm{C}$ for 24 hours. Following the curing process, the PDMS surface was refreshed in a $20 \% \mathrm{HCL}$ solution for $8 \mathrm{~min}$. to remove any contaminates and then placed in an oxygen plasma for $1 \mathrm{~min}$ at an RF power of 100 watts with $\mathrm{O}_{2}$ flow at $300 \mathrm{sccm}$ to activate the surface. The metal traces were patterned onto PDMS using a lift-off process. In this process, photoresist was photolithographically patterned on the surface, as seen in Fig. 5.3e, using a glass photomask. A chlorobenzene soak was used prior to developing to swell the edge profile of the features in order to facilitate the lift-off process. After developing, the exposed PDMS surface was again refreshed in HCL and placed in an oxygen plasma to reactivate the surface in preparation for metal deposition. A $5 \mathrm{~nm}$ adhesion layer of titanium, a $200 \mathrm{~nm}$ layer of gold, and another $5 \mathrm{~nm}$ layer of titanium were deposited at $0.1 \mathrm{~nm} / \mathrm{sec}$ over the patterned photoresist, adhering to the PDMS in regions where the photoresist was removed. The third layer of metal, $5 \mathrm{~nm}$ titanium, was to prompt adhesion of the metal to the subsequent PDMS passivation layer. Removal of the resist and the excess metals in an 
acetone bath revealed the desired pattern, as illustrated in Fig. 5.3f. The metal traces were then passivated selectively using membrane transfer rather than patterning and spinning the PDMS directly onto the metal traces. The membrane transfer method (See chapter 3 section 2.7) required patterning a PDMS film on a flexible substrate such as polyimide and then aligning and bonding to the initial PDMS layer with metal traces as shown in Fig. 5.3g. Following the passivation process, platinum electrodes were deposited either by electroplating or by alternative approaches under development at LLNL (see Fig. 5.3h). For electroplating, devices were sent to Jack Judy's group at UCLA. Once the process was completed the devices were diced and removed from the wafer, Fig. 5.3i.
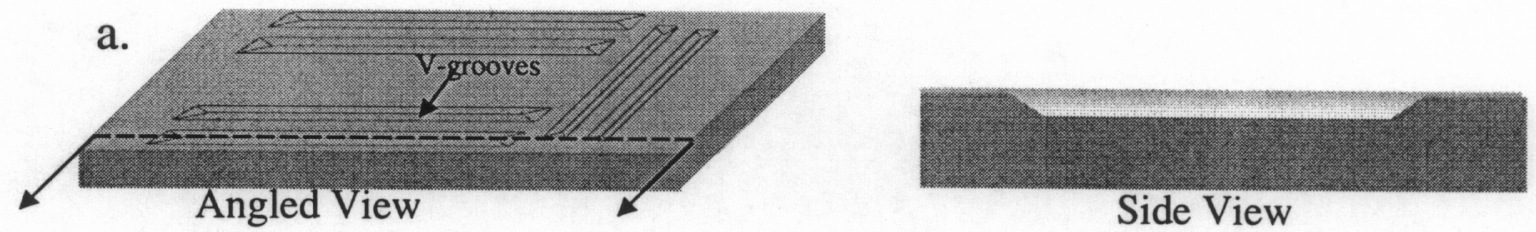

Side View

b.

b.

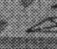

\section{Angled View}

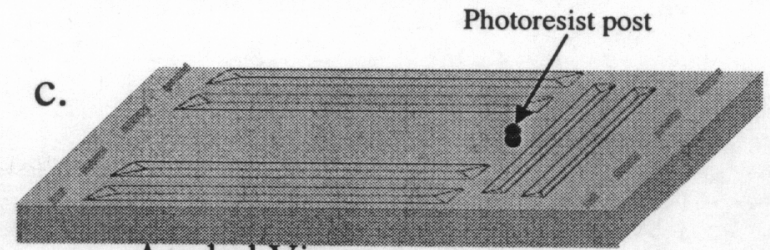

Angled View

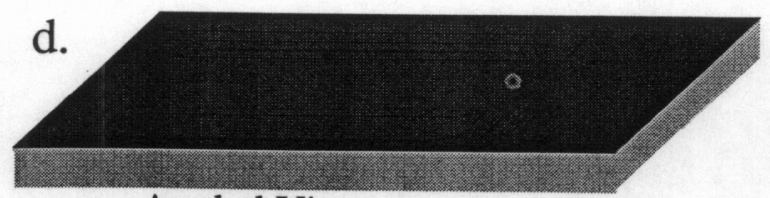

Angled View

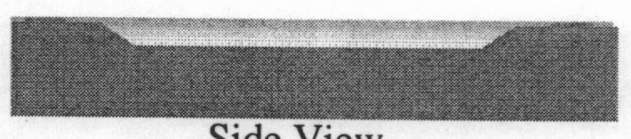

Side View

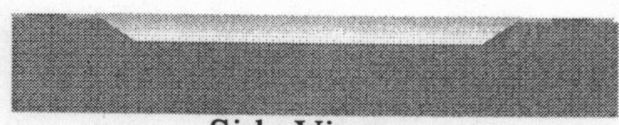

Side View

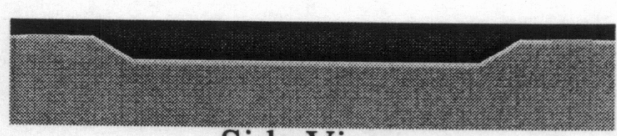

Side View 


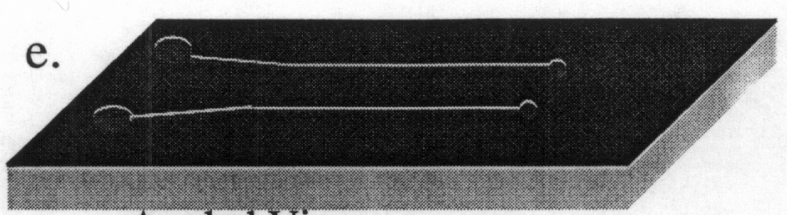

Angled View

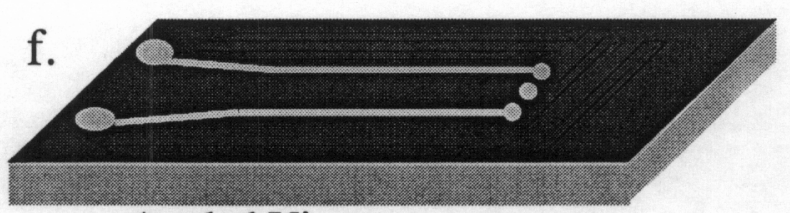

Angled View

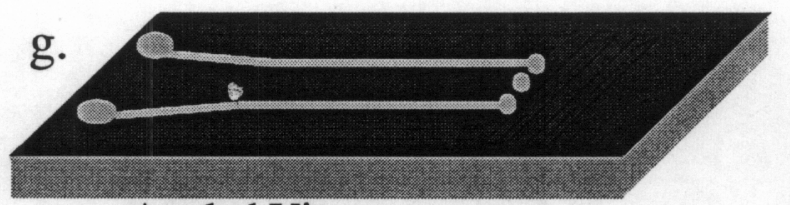

Angled View

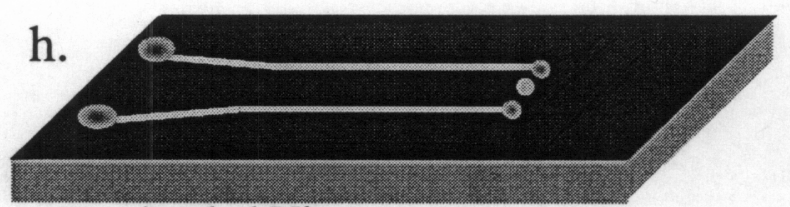

Angled View

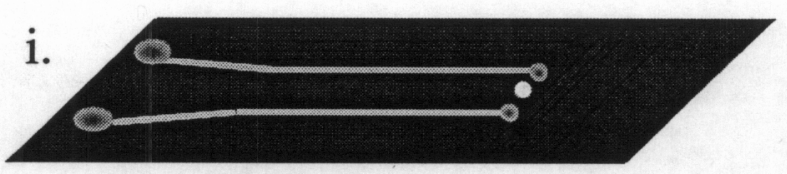

Angled View

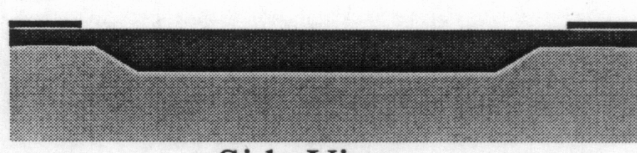

Side View

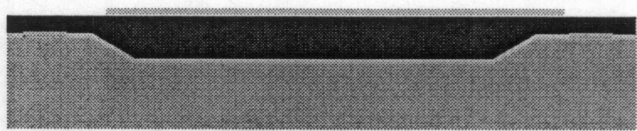

Side View

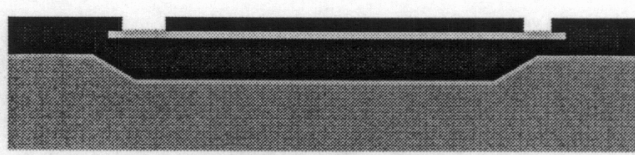

Side View

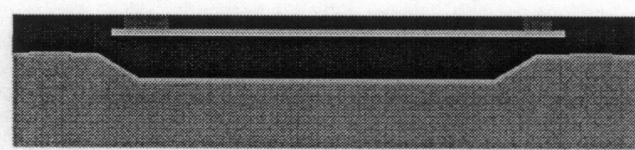

Side View

\section{Side View}

Figure 5.3. Process flow for $2^{\text {nd }}$ generation devices (a) etch v-grooves in silicon substrate, coat with gold (b) etch gold in selected regions (c) pattern photoresist for tack holes (d) spin on PDMS, allow to planarize (e) pattern photoresist for lift-off (f) deposit and pattern Ti/Au/Ti film (g) perform membrane transfer for passivation layer (h) deposit platinum electrodes (i) dice and remove from the wafer

\subsection{EXPERMENTAL APPROACH}

\subsubsection{Adhesion tape test}


The adhesion tape test was performed on all wafers as described in chapter 4 section 3.2. No metal can be visually detected on the tape in order for a wafer to pass adhesion test. In addition, three sets of experiments with various surface treatments were conducted at three different curing times: 1,24 , and 48 hours. These experiments were performed to study the effects of various surface treatments on metal adhesion to the PDMS surface. In set-1, PDMS was metalized with no $\mathrm{HCL}$ or $\mathrm{O}_{2}$ plasma treatments. In set-1 3 wafers were fabricated and tested, one at each cure time 1,24 , and 48 . Set-2 underwent $\mathrm{O}_{2}$ plasma treatment, but no HCL treatment. In set-2 15 wafers were fabricated and tested, five at each cure time. In set- 3 both $\mathrm{O}_{2}$ plasma treatment and $20 \%$ HCL treatment were carried out. In set-3 15 wafers were fabricated and tested, five at each cure time. All wafer were cured at $66^{\circ} \mathrm{C}$.

\subsubsection{Electrical continuity}

Electrical continuity was conducted on all devices on the wafers used for the adhesion tape test as described in chapter 4 section 3.3. Each of the wafers tested included 26 devices. In set-1, total number of devices tested 78 and for set- 2 and set-3 the total number of devices tested were 390 for each set.

\subsubsection{Interfacing to Electronics}

From the animal experiments (see chapter 4 section 3.6), we found that making electrical contact is one of the most challenging issues associated with testing of the devices. To address this problem, electrical interconnects were developed to enable quick and reliable interfacing with the microelectrode array. Two separate designs were 
fabricated. One design was fabricated using PC board technology and another design was fabricated on glass using traditional photolithography. The PC board interconnect was comprised of two styles. One style had electroplated bumps for making uniform contact to the metal contact pads on the PDMS devices and the other style had pins that would go through the PDMS devices (Fig. 5.11). The purpose of the pins was to aid in aligning the interconnect to the contact pads. The second interconnect design was fabricated on glass, since PDMS adheres nicely to glass. The glass interconnect design was bulkier than the PC board interconnect design to allow it to interface with a conventional edge connector to aid in testing of electrical continuity and to perform more extensive soak tests on the device. The PC board interconnect was more compact and required custom connectors to mate with electronics, but it would be more favorable for implantation purposes. The PC board interconnects were fabricated at LLNL's circuitry machine shop.

\subsubsection{GLASS INTERCONNECT FABRICATION}

Clean glass wafers were coated with gold using chromium as the adhesion layer. Then using traditional photolithography, photoresist is spun, exposed and developed [2]. The desired pattern seen in Fig. 5.4 is obtained by etching back the metal layer using the proper etchants [2]. The glass was then diced to produce individual interconnects. 
PC board interconnect design

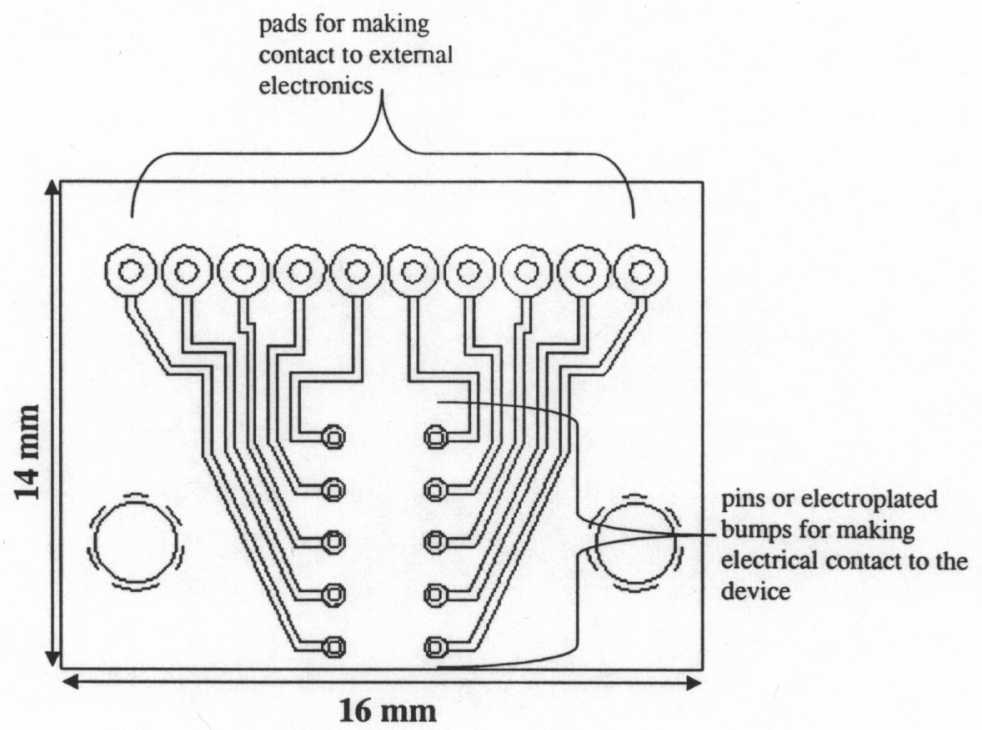

Glass interconnect design

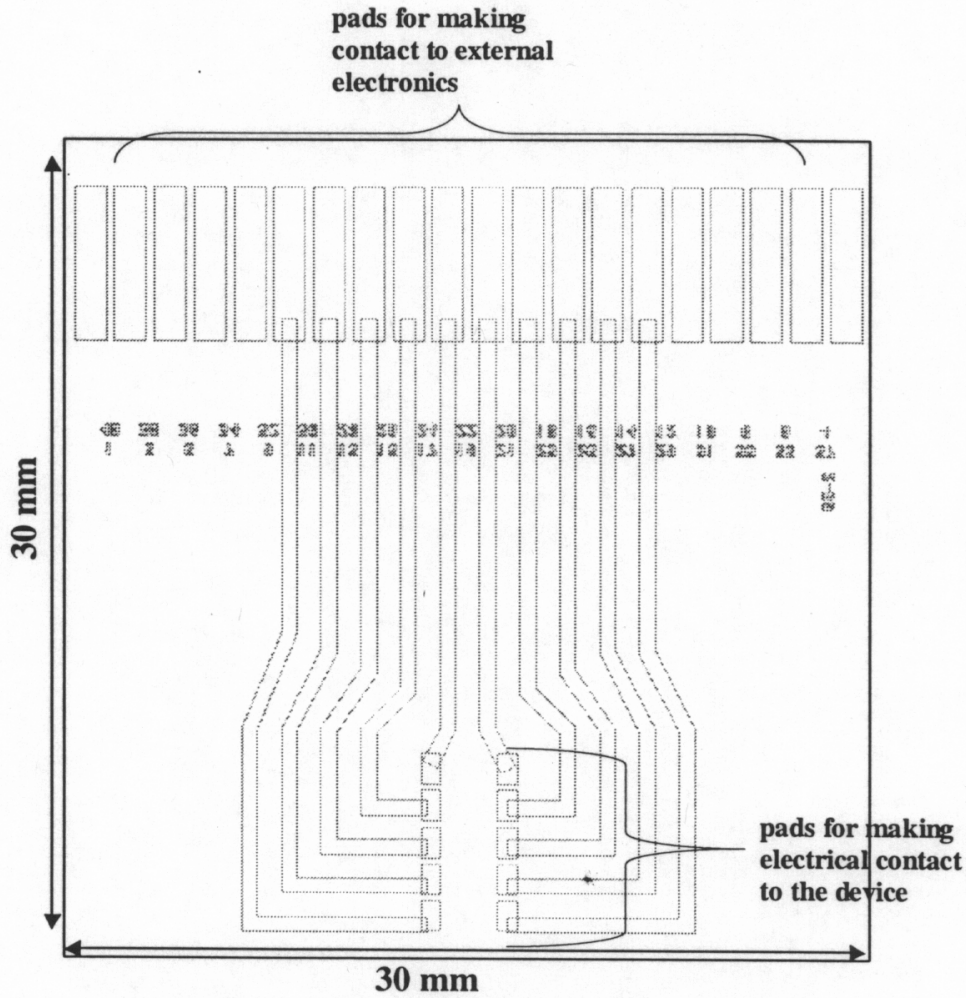

Figure 5.4. $\mathrm{PC}$ board and glass interconnect design 


\subsubsection{Soak Test Experiments}

Soak tests were conducted on the $2^{\text {nd }}$ generation devices to determine if the passivation layer is functioning properly by insulating the metal traces and preventing leakage. The samples were soaked in phosphate buffered saline (PBS) at room temperature for 5 months at LLNL. Soak tests were performed on metalized PDMS samples that were completely passivated, selectively passivated and completely unpassivated. The completely passivated samples, shown in Fig. 5.5a, were used to determine if there is any leakage through the PDMS passivation layer. The selectively passivated samples (Fig. 5.5b) were to determine if any leakage occurs at the electrode PDMS passivation interface and between the PDMS to PDMS bond. The completely unpassivated samples, Fig. 5.5c, serve as a positive control. The metal traces in each sample were examined visually in designated areas for any signs of leakage and corrosion.

a.

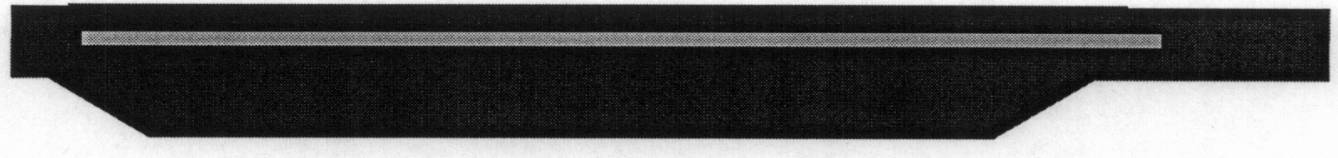

b.

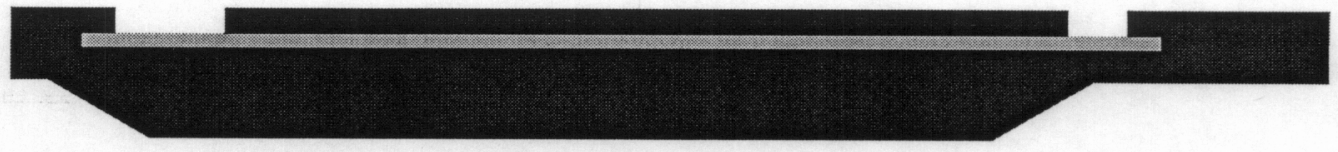

c.

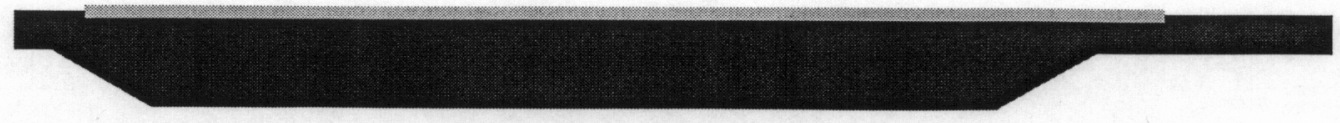

Figure 5.5. a. completely passivated b. selectively passivated c. completely unpassivated 


\subsubsection{Chronic Surgical Implantation Experiments}

Dr. Dilek Guven was the surgeon in Dr. Mark Humayun's group at USC who conducted the surgical implantation procedure. A $2^{\text {nd }}$ generation device was implanted on April $15^{\text {th }}, 2003$ into a canine's eye. This was a chronic implantation, meaning the implant will remain in the eye for a prolonged period to time. In this case the implant will remain in the eye for a 6 months observation period.

\subsubsection{CHRONIC IMPLANT}

The device that was used for the implantation is the stimulation electrode style that was completely passivated with no exposed electrodes or contact pads (see Fig. 5.6). The implant was performed in a canine eye, which is approximately the size of a human eye. The objective of this implantation is to determine: 1) handling and surgical manipulation procedures 2) how well the device conforms to the retina using optical coherence tomography (OCT) 3) the mechanical effects of the device on the retina and 4) the biocompatibility issues. The handling and surgical procedures were determined by the physicians. Contact uniformity was determined using OCT, by measuring the gap between the device and the retina. The OCT is a relatively new technique for highresolution cross-sectional imaging. This technique is analogous to ultrasound technology by which distance information is extracted from the time delays of reflected signals. The use of optical rather than acoustic waves results in higher longitudinal resolution [3]. As a control an OCT scan of the retina was made prior to the implantation procedure. One week after implantation OCT scans were made with the device lying on the retina. The condition of the retina is monitored visually through weekly ophthalmologic 
examinations to inspect for mechanical or biocompatibility effects. Histopathologic examination of the retinal tissue and evaluation of the metal traces will be made post explanation.

\subsection{Surgical procedures for chronic implant}

Approximately one-third of the prototype device was inserted into the canine eye as seen in Fig. 5.12. All procedures were approved by the IACUC (Institute of Animal Care Use Committee) of the University of Southern California. The canine was placed under general anesthesia for the surgical procedure. The device was implanted epiretinal into the eye of one canine, following standard vitrectomy procedure. The procedure began with detaching the musculature around the eyeball and placing traction sutures in the rectus muscles for maneuvering the eyeball. An infusion cannula was inserted to allow for aspiration of the vitreous and to provide a port for saline to be pumped through continuously to maintain ocular pressure for the duration of the procedure. The pupil was dilated using phenylephrine $2.5 \%$ and a magnifying lens ring was sutured on top. The magnifying lens, in combination with the surgical microscope aided the surgeon in seeing clearly in side the eye during the procedure. A sclerotomy, an incision through the sclera, was made on one side of the eye to allow for device insertion. The rib area at the edge of the device was grasped using a microforceps with teeth and inserted into the eye, some more length was inserted using another forceps from outside part of the device. Once inside the eye another sclerotomy was made on the opposite side and tack holder with the tack loaded was inserted into the eye. The tack was inserted in the tack hole and used to maneuver and position the device before attaching it to the retina supero-temporally. Following the placement of the device the sclera was sutured and the remaining part of 
the device was place underneath the musculature. The infusion line was disconnected and the traction sutures were removed. Postoperatively, both systemic and topical steroid and antibiotic were applied.

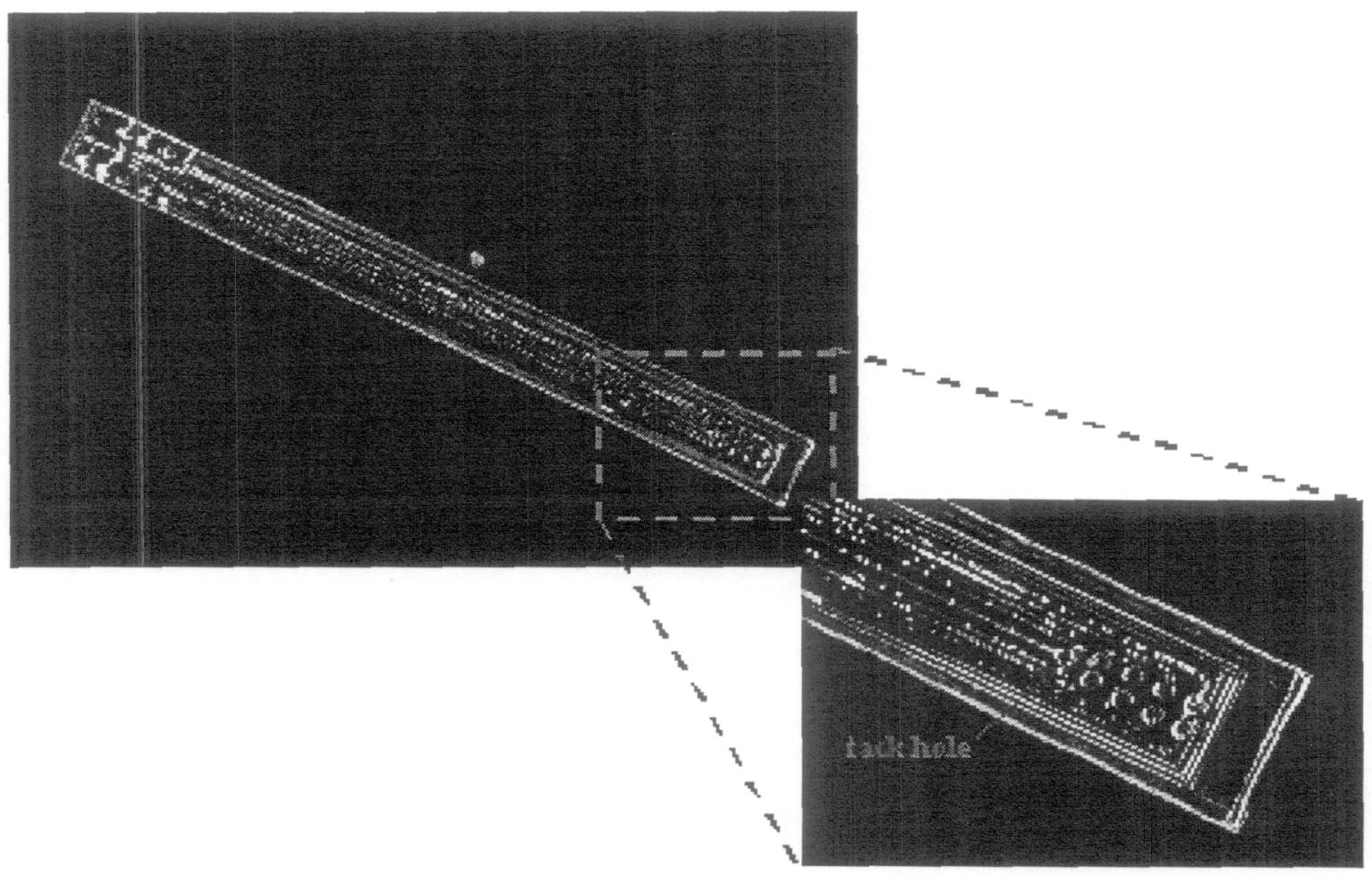

Figure 5.6. Evaluation prototype of $2^{\text {nd }}$ generation surgical implantation device with a close-up illustrating placement of the tack hole

\subsection{RESULTS}

\subsubsection{Fabrication Results}

A number of measures were undertaken to improve the device yield and to overcome problems encountered during the $1^{\text {st }}$ generation device fabrication process. These measures included 1) using a thinner photoresist ( $A Z^{\circledR} 1518$, Clariant) for the metalization process, 2) curing the PDMS for 24 hrs at $66^{\circ} \mathrm{C}, 3$ ) soaking in $20 \% \mathrm{HCL}$ for 
8 min to refresh the PDMS surface, and 4) cleaning the oxygen plasma chamber prior to each run. All of these measures were to minimize surface contamination (see chapter 6). Other modifications were made to provide structural integrity and facilitate surgical implantation of the devices. These modifications were the micro-molded ribs and the tack hole.

Retinal tack hole was patterned in the PDMS device as seen in Fig. 5.6. This allowed for placement of the retinal tack without ripping or damaging the device during implantation. The molded ribs along the outer perimeter as seen in Fig. 5.7, facilitated surgical implantation by providing an area that can be grasped by the forceps. Metal traces were deposited on the PDMS surface as seen in the SEM pictures in Fig. 5.8. The thinner Ti layer provided less stress in the metal traces (see chapter 7). To minimize damage to the metal traces by the passivation layer, membrane transfer was used for selective passivation (see Fig. 5.10), which resulted in higher yield (see Table 5.1). Andy Hung at UCLA plated platinum electrodes onto the wrinkled metalized PDMS surface. This resulted in desirable high surface area electrodes; however the stresses in the platinum and PDMS surface contamination caused the electrodes to crack and flak upon handling and uneven plating respectively. 

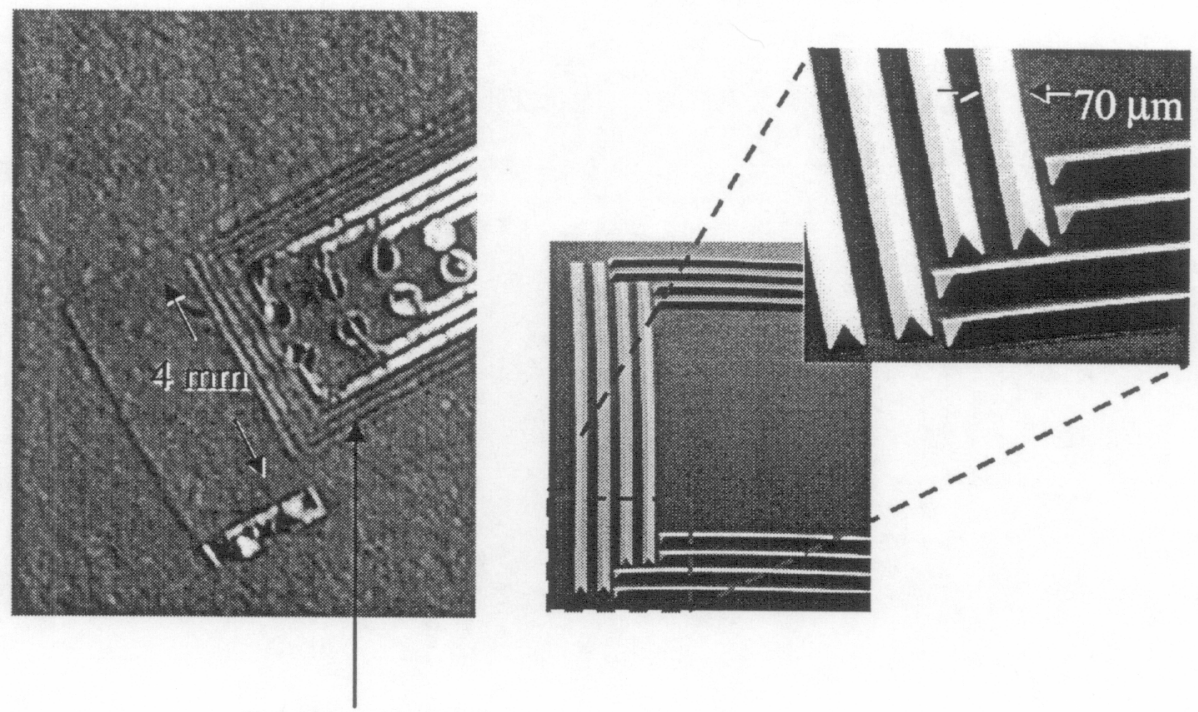

Reinforcement Ribs on the backside of the device

Figure 5.7. Close-up of the electrode region of a $2^{\text {nd }}$ generation microelectrode array (left), and SEM images of the backside of the microelectrode array showing the micromolded reinforcing ribs.
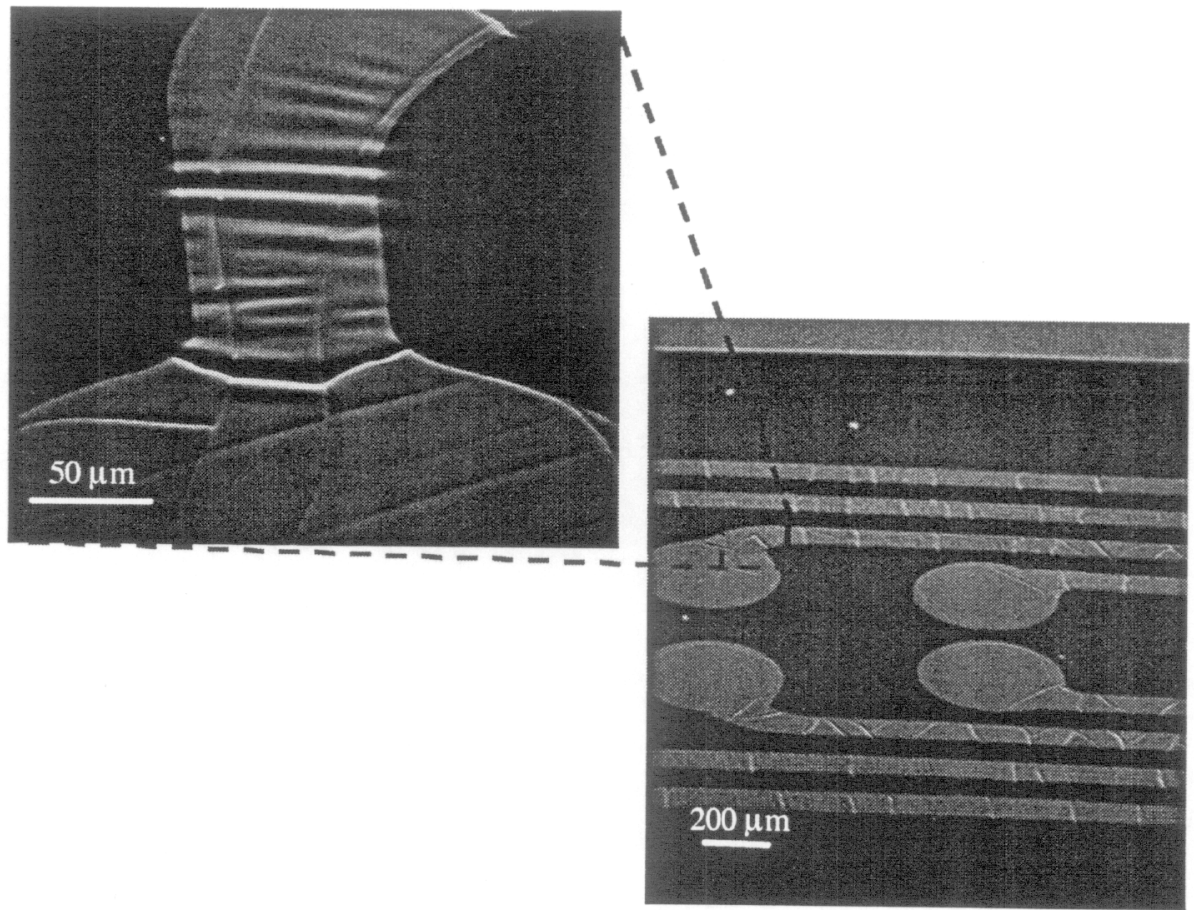

Figure 5.8. SEM of deposited metal traces on PDMS 

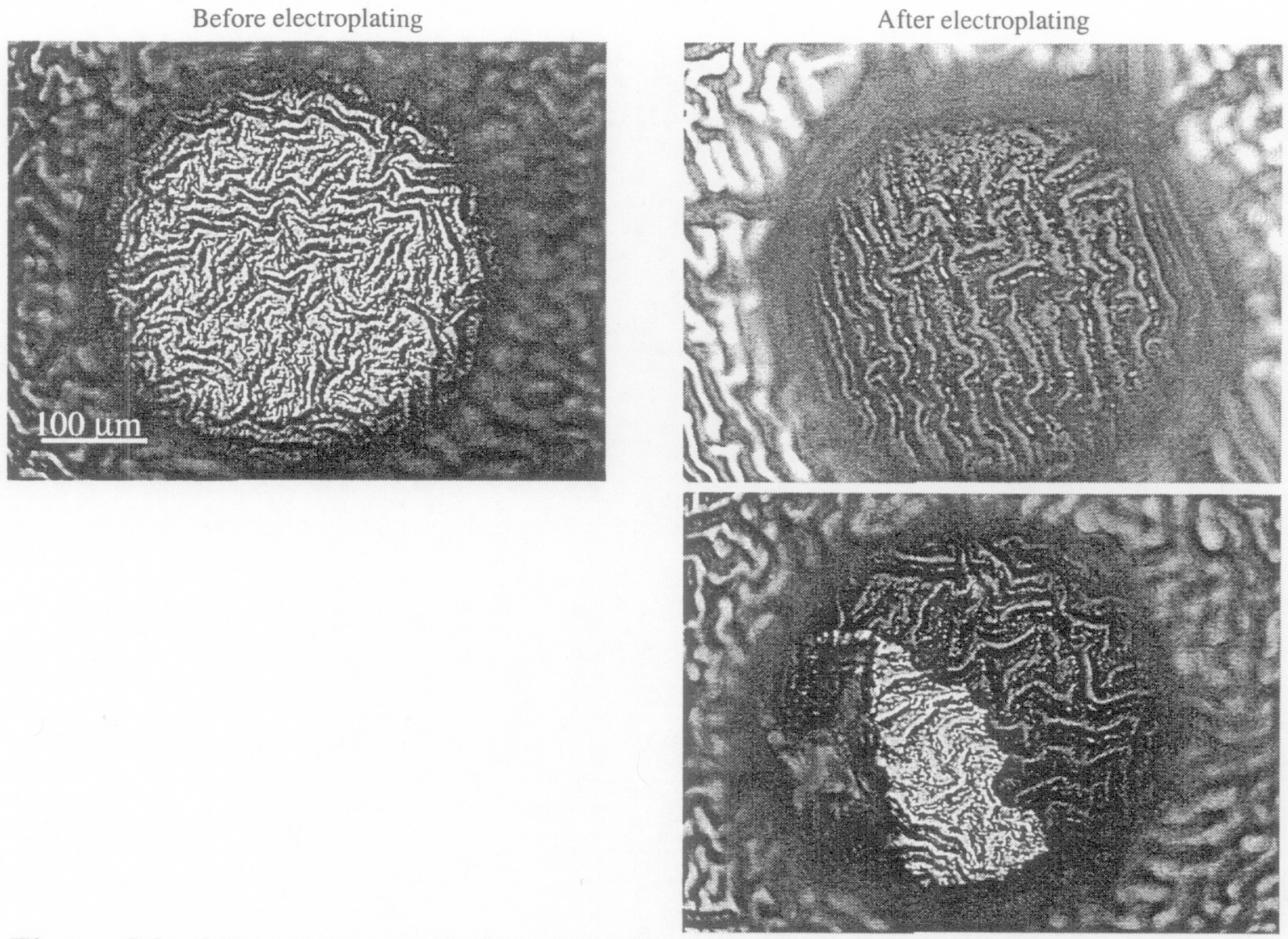

Figure 5.9. Microelectrodes before with $\mathrm{Ti} / \mathrm{Au}$ seed layer and after $\mathrm{Pt}$. electroplating images

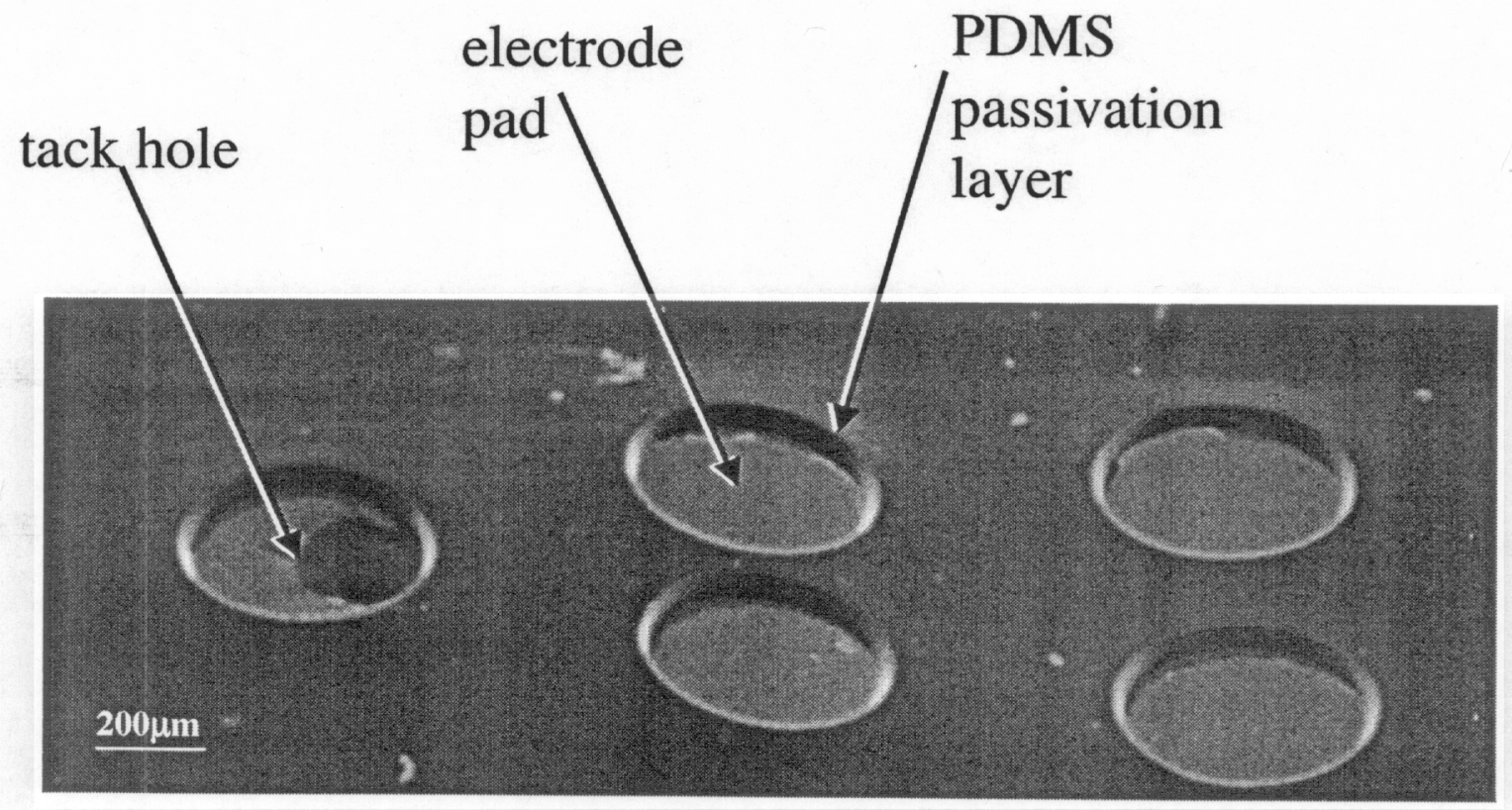

Figure 5.10. SEM of selective passivation using membrane transfer approach 


\subsubsection{Adhesion and Electrical Continuity Results}

Table 5.1 presents adhesion and electrical continuity results based on different surface treatments and curing times. Set-1 results had the lowest success in metal adhesion and device yield. However when both oxygen and HCL treatments were performed along with longer curing times both adhesion and electrical continuity results increased significantly. For the 24 and 48 hour cure $\mathrm{O}_{2}$ treated samples in set-2, there was a $33 \%$ increase for both, in successful metal adhesion over the 1 hour cure samples in the same set. The was an increase in device yield, for the 24 and 48 hour cure in set-2, by 23 and $14 \%$ for the unpassivated devices and 242 and $219 \%$ for the passivated devices over the 1 hour cure devices. In addition to the $\mathrm{O}_{2}$ treatment in set-2, set- 3 included HCL treatment. This resulted in a $25 \%$ increase of adhesion over set- 2 for the 24 and 48 hour cure. The yield for set- 3 increased by 10 and $15 \%$ for unpassivated devices and 18 and $28 \%$ for passivated devices in comparison to the 24 and 48 hour cures in set-2 respectively. The one-hour cure samples in set-2 and the 24-hour cure samples in set-3 represent $1^{\text {st }}$ generation and $2^{\text {nd }}$ generation devices respectively. Comparing the 24 hour cure of set- 3 results to the 1 hour cure in set- 2 results reveals that adhesion increased by $67 \%$ and device yield increased by $35 \%$ for unpassivated and by approximately $300 \%$ for passivated 
Table 5.1. Adhesion and electrical continuity results

\begin{tabular}{|c|c|c|c|c|c|c|}
\hline & $\begin{array}{l}\text { Adhesion } \\
\text { Test } 1^{\text {a,1 }}\end{array}$ & $\begin{array}{l}\text { Electrical } \\
\text { continuity } \\
1^{b, 1}\end{array}$ & $\begin{array}{l}\text { Adhesion } \\
\text { Test } 2^{\text {a, } 2}\end{array}$ & $\begin{array}{l}\text { Electrical } \\
\text { continuity } \\
2^{b, 2}\end{array}$ & $\begin{array}{l}\text { Adhesion } \\
\text { Test } 3^{\text {a, } 3}\end{array}$ & $\begin{array}{l}\text { Electrical } \\
\text { continuity } \\
3^{b, 3}\end{array}$ \\
\hline \multicolumn{7}{|l|}{1 hr. cure } \\
\hline Unpassivated & $\begin{array}{l}0 \% \\
\text { passed }\end{array}$ & $0 \%$ passed & $\begin{array}{l}60 \% \\
\text { passed }\end{array}$ & $\begin{array}{l}66 \% \\
\text { passed }\end{array}$ & NA & NA \\
\hline Passivated $^{c}$ & $\mathrm{NA}$ & $\mathrm{NA}$ & NA & $\begin{array}{l}21 \% \\
\text { passed }\end{array}$ & $\mathrm{NA}$ & NA \\
\hline \multicolumn{7}{|l|}{24 hr. cure ${ }^{*}$} \\
\hline Unpassivated & $\begin{array}{l}0 \% \\
\text { passed }\end{array}$ & $\begin{array}{l}15 \% \\
\text { passed }\end{array}$ & $\begin{array}{l}80 \% \\
\text { passed }\end{array}$ & $\begin{array}{l}81 \% \\
\text { passed }\end{array}$ & $100 \%$ passed & $\begin{array}{l}89 \% \\
\text { passed }\end{array}$ \\
\hline Passivated $^{d}$ & $\mathrm{NA}$ & NA & NA & $\begin{array}{l}72 \% \\
\text { passed }\end{array}$ & $\mathrm{NA}$ & $\begin{array}{l}85 \% \\
\text { passed }\end{array}$ \\
\hline \multicolumn{7}{|l|}{48 hr. cure } \\
\hline Unpassivated & NA & $\mathrm{NA}$ & $\begin{array}{l}80 \% \\
\text { passed }\end{array}$ & $\begin{array}{l}75 \% \\
\text { passed }\end{array}$ & $100 \%$ passed & $\begin{array}{l}86 \% \\
\text { passed }\end{array}$ \\
\hline Passivated $^{d}$ & $\mathrm{NA}$ & $\mathrm{NA}$ & NA & $\begin{array}{l}67 \% \\
\text { passed }\end{array}$ & NA & $\begin{array}{l}86 \% \\
\text { passed }\end{array}$ \\
\hline
\end{tabular}

${ }^{a}$ Adhesion Test is performed per wafer

${ }^{b}$ Electrical continuity is performed per device with 26 devices per wafer

${ }^{c}$ Passivated by spinning on PDMS directly onto the metal traces

${ }^{\mathrm{d}}$ Passivated by using membrane transfer approach

${ }^{1}$ Set-1: Adhesion Test 1 and Electrical continuity 1 wafers had no $\mathrm{HCL}$ or $\mathrm{O}_{2}$ treatment

${ }^{2}$ Set-2: Adhesion Test 2 and Electrical continuity 2 wafers had $\mathrm{O}_{2}$ treatment, but no $\mathrm{HCL}$ treatment

${ }^{3}$ Set-3: Adhesion Test 3 and Electrical continuity 3 wafers had both HCL and $\mathrm{O}_{2}$ treatment

${ }^{*}$ Cure temperature $66^{\circ} \mathrm{C}$

\subsubsection{Electronic Interconnect Results}

Both the PC board interconnects and the glass interconnect are shown in Fig. 5.11. Both styles of PC board connectors were difficult to align and make reliable contact with the PDMS devices. The glass interconnects required little effort to align and bond the PDMS devices to. 


\section{PC board interconnect}

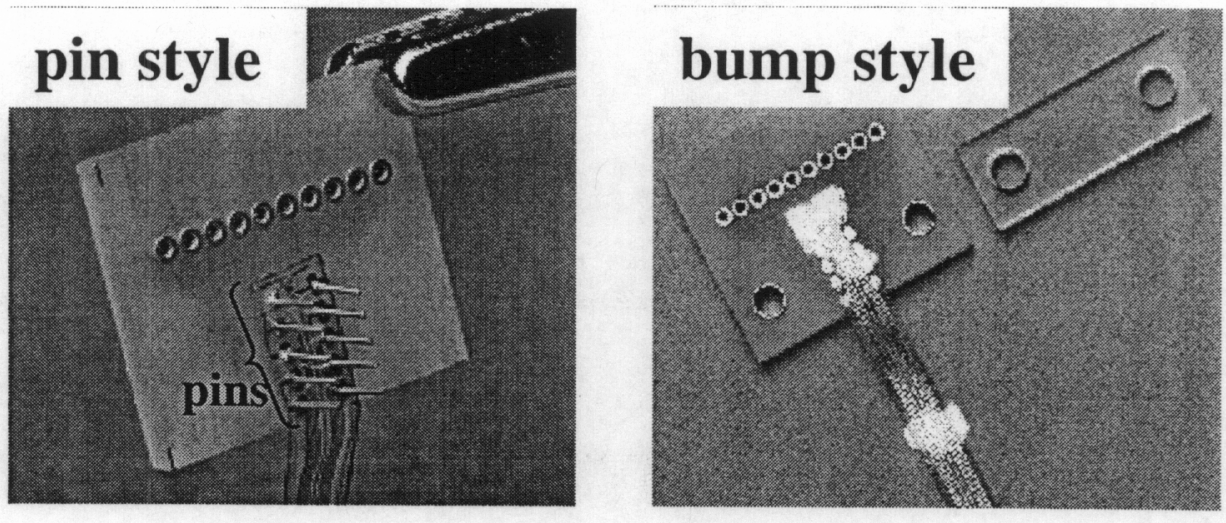

\section{Glass interconnect}

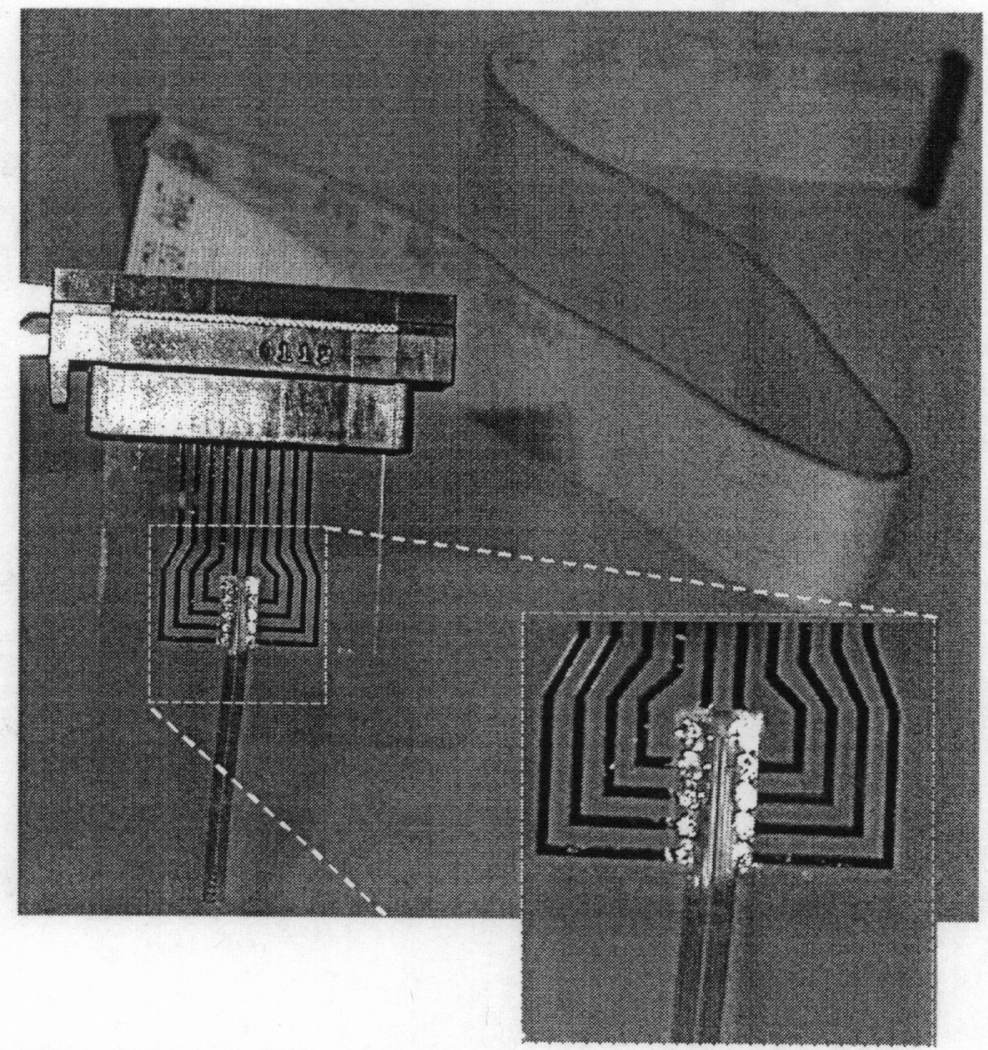

Figure 5.11. Electrical interconnect

\subsection{4 $2^{\text {nd }}$ Generation Soak Test Results}

Ongoing soak test results have shown that the completely passivated metal traces exhibit no detectable leakage after nearly 5 months of testing with no apparent signs of 
degradation. There was evident fluid leakage in some of the selectively passivated devices that had only $\mathrm{Ti} / \mathrm{Au}$ metal traces. However no leakage was detected for the $\mathrm{Ti} / \mathrm{Au} / \mathrm{Ti}$ metal selectively passivated samples. The completely unpassivated devices have not shown any signs of corrosion; however, further examination is needed to determine long-term effects.

\subsubsection{Chronic Surgical Implant Results}

On April $15^{\text {th }}, 2003$ a $2^{\text {nd }}$ generation device was successfully implanted into a dog's eye. No hemorrhage or retinal fold could be seen at that time of insertion. However, the leading edge of the device looked a little elevated following tack insertion. Images of the insertion procedure are shown in Fig. 5.12. One week later OCT scans were taken of the retina (see Fig. 5.13). Flowing the shadows caused by the metal electrodes and traces onto the retina from the OCT scans, it can be seen that the device is conforming the retina. Also from the OCT scans it can be seen that a slight neurosensory detachment beneath the device occurred postoperatively. According to the surgeon who performed the implantation, Dr. Dilek Guven, this detachment may be induced by fluid collection due to the tack insertion. Despite this detachment, the array remains conformal to the inner surface of the retina. 

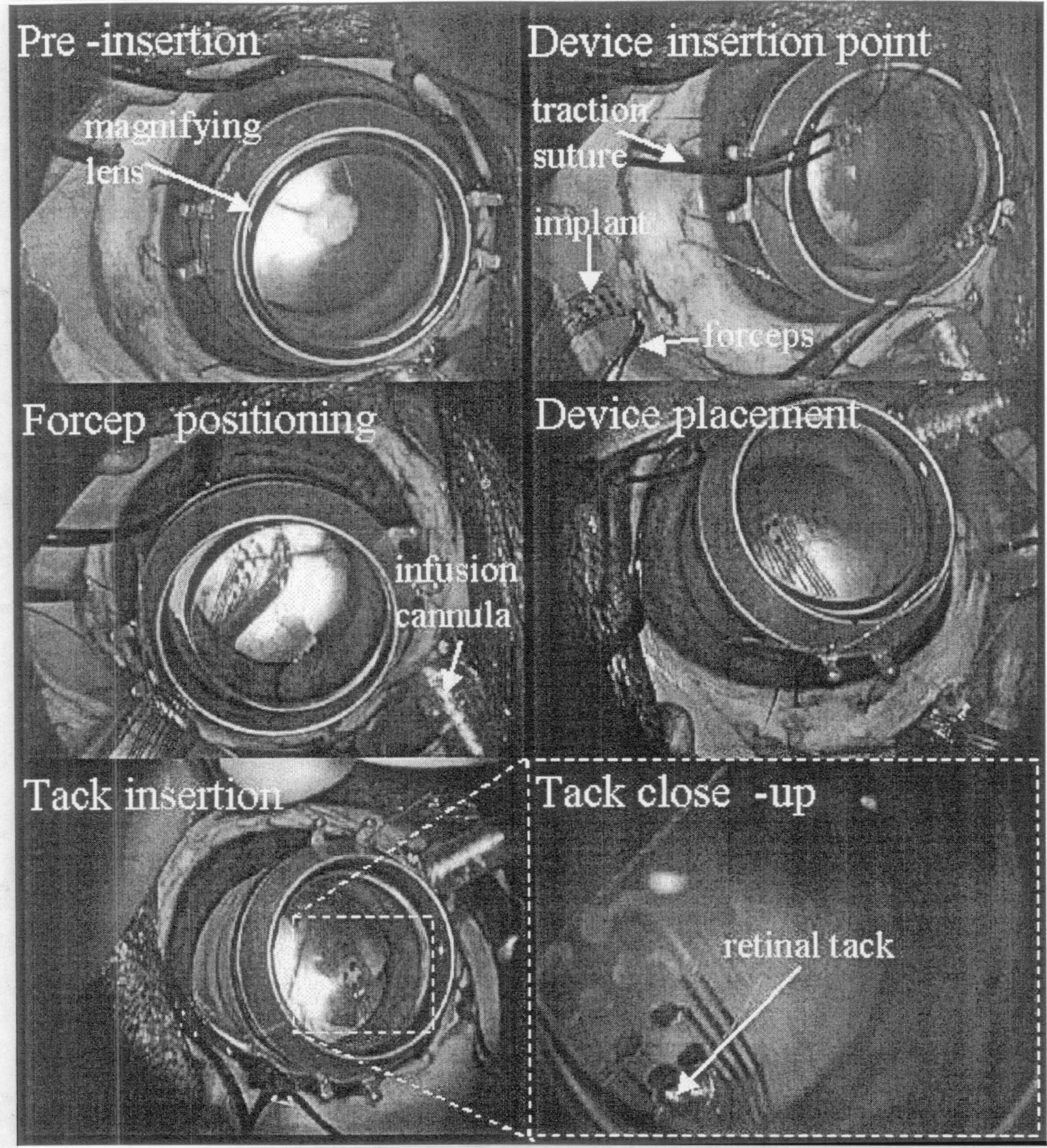

Figure 5.12. $2^{\text {nd }}$ generation chronic implant 


\section{OCT Scanned Image}
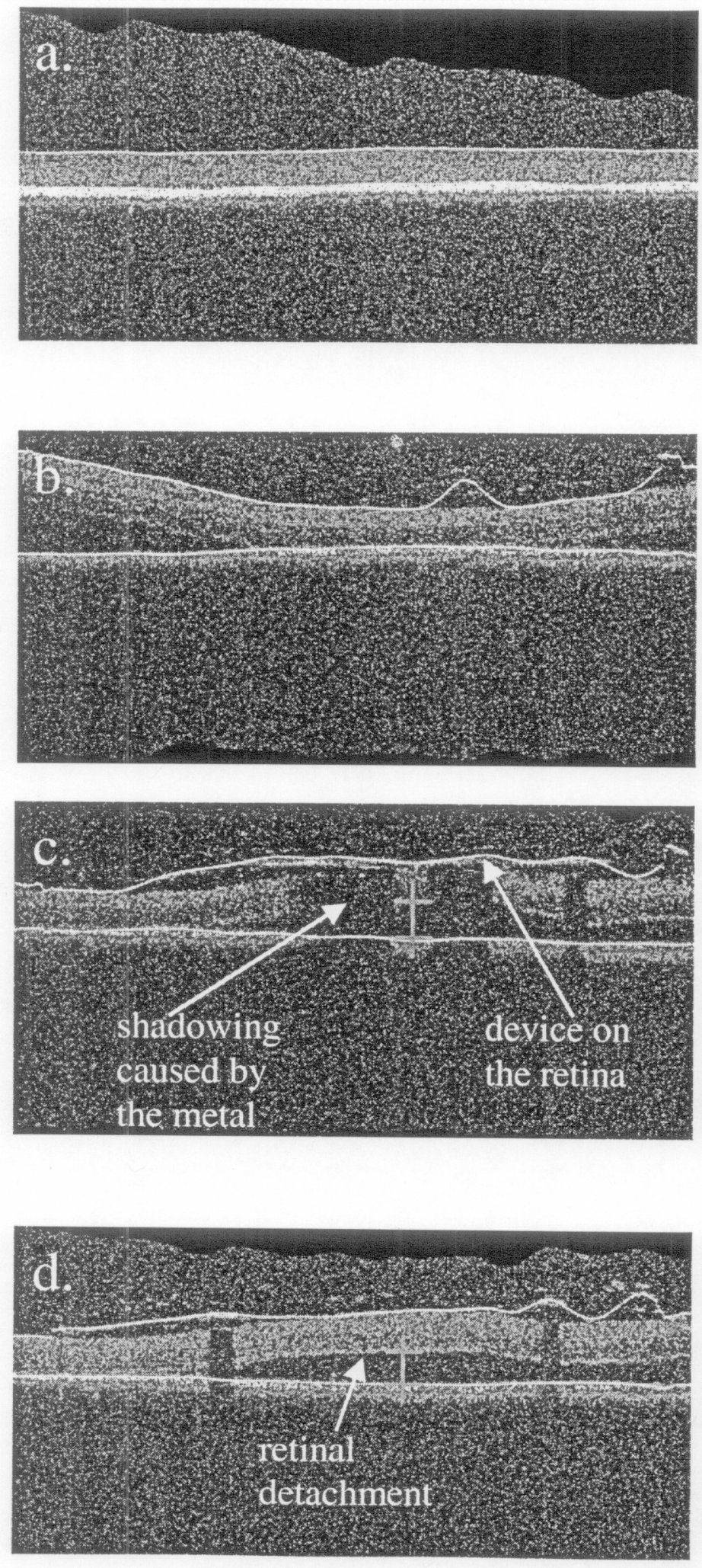

\section{Video Image}
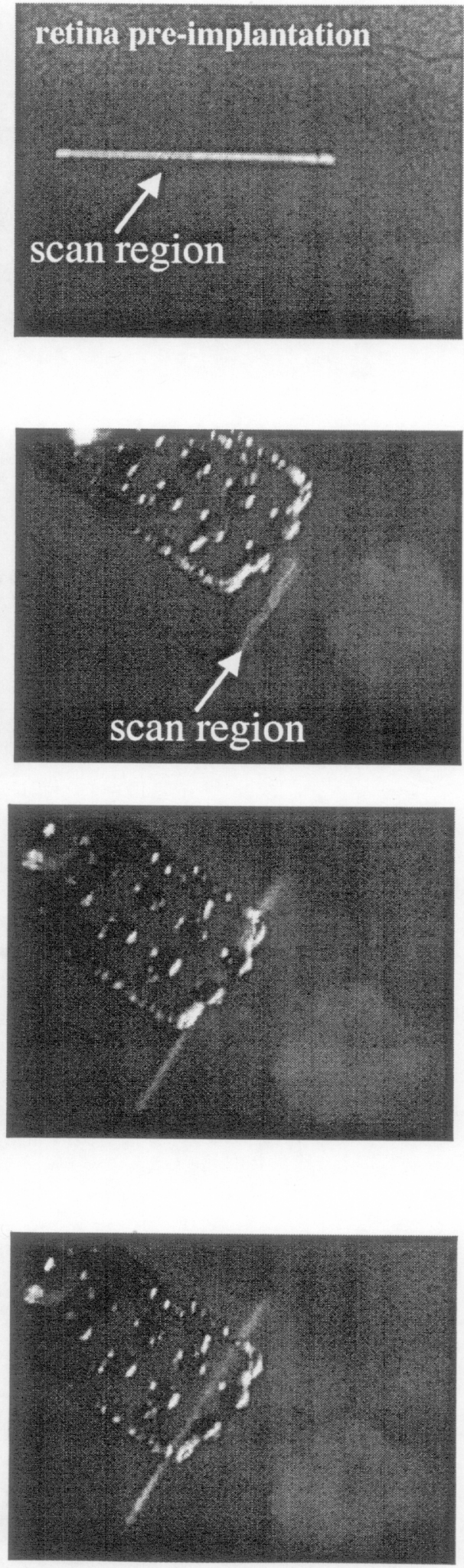

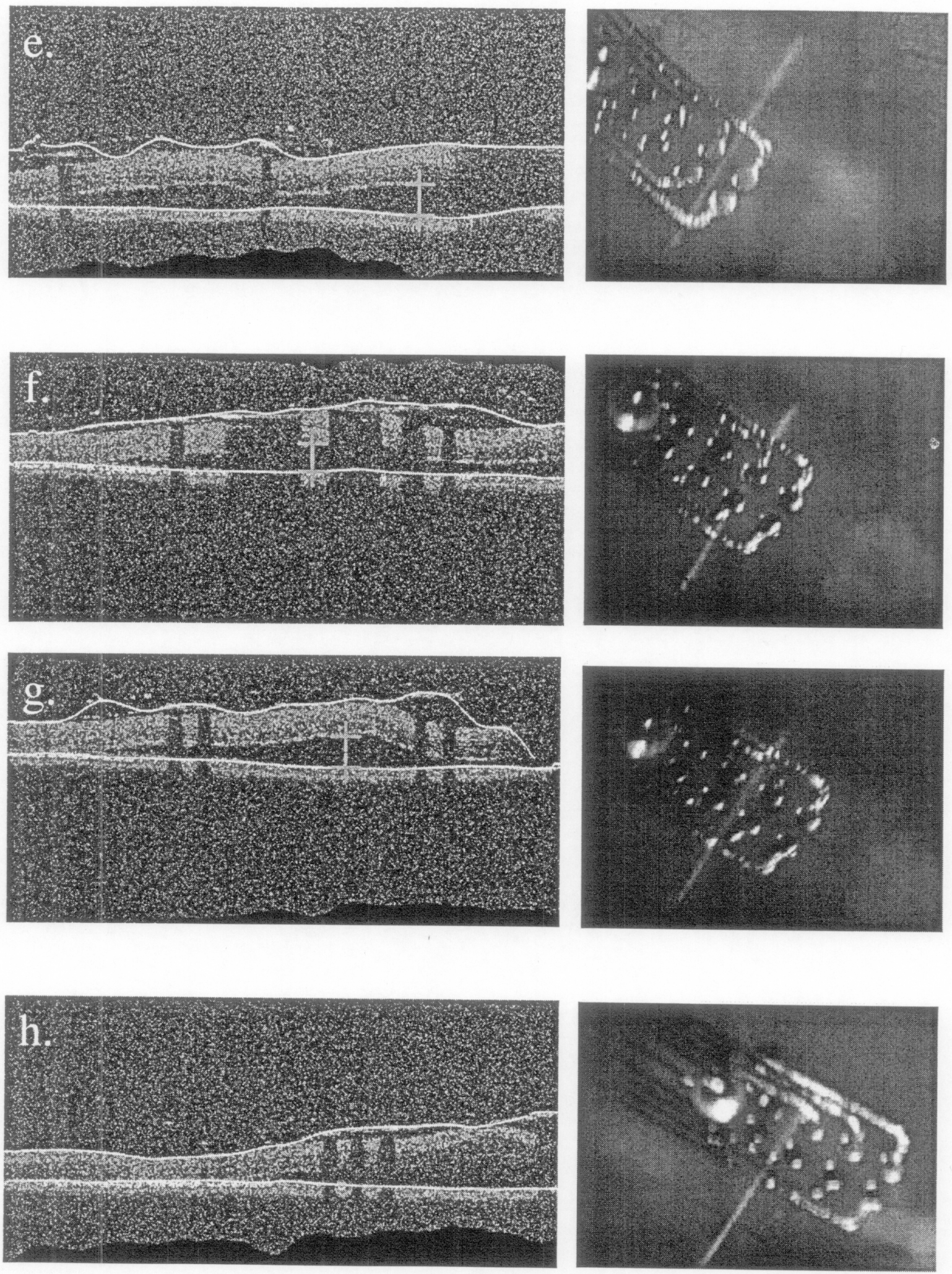

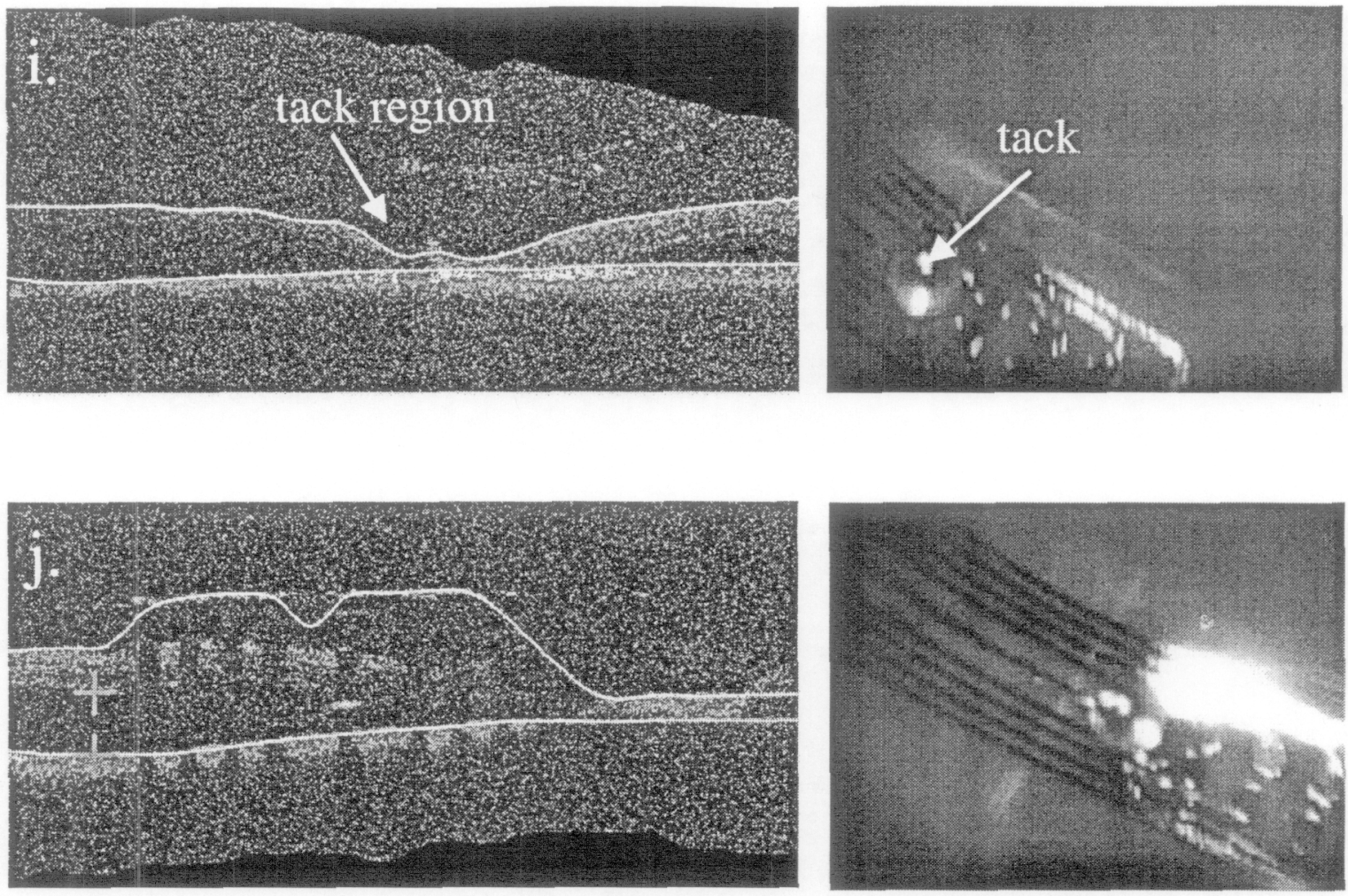

Figure 5.13. Scanned OCT images on the left and video images on the right. The white line in the OCT images represent the PDMS device. The white light in the video image is the scan region.

\subsection{DISCUSSION}

The design modifications and process refinements made in the $2^{\text {nd }}$ generation significantly increased device yield and fabrication repeatability. This is indicated from the adhesion and electrical continuity results presented in Table 5.1. There is a $67 \%$ increase in successful metal adhesion and approximately a $300 \%$ increase in device yield in the $2^{\text {nd }}$ generation devices in comparison to the $1^{\text {st }}$ generation devices. The micromolded ribs enhanced the surgical implantation process by providing a region that can be grasped by the forceps during insertion and maneuvering. The ribs also provided structural support, which minimized the curling and flimsiness of the device, facilitating 
handling. The ribs generated a slight curvature in the device, which aided the device in conforming more uniformly to the retina as well as lessening the force induced on the retinal. In the future the ribs can be tailored to customize the curvature of the device to match the curvature of the eye for a best-fit device. The tack hole was used as a means to drag and reposition the device before fastening it onto the retina using a metal tack. The placement of the tack is thought to have caused the retina to detach underneath the device, as seen in the OCT scans in Fig. 5.13. Since the PDMS device is so conformal we believe that less forceful means, such as biocompatible fibrin adhesives, can be sufficient in maintaining the device onto the retina. Despite the retinal detachment, the images from the OCT scans revealed that the device was conform uniformly onto the retina. This is significant for the development and design of an implant, since the distance between the electrodes and the target tissue influences the efficacy of electrical stimulation [4]. The modifications incorporated into the $2^{\text {nd }}$ generation device facilitated the surgical implantation procedure in terms of insertion and handling.

Platinum electrodes were electroplated onto the wrinkled metal traces to provide high surface area electrodes. However the combination of intrinsic stresses in the platinum and PDMS surface contamination remains problematic, causing cracks, flaking, and uneven plating of the electrodes, as seen in Fig. 5.9. Other avenues at LLNL are being pursued to develop sturdy and reliable electrodes that will meet the required needs. Preliminary results from the soak test indicate that PDMS is an appropriate material for this application. However, accelerated long-term soak test studies are vital to determine the viability of the devices for used as a permanent implant. 
The development of the electrical connector can facilitate testing and minimize damage to the device caused by direct probing of the contact pads. The PC board interconnect was an attractive candidate for long-term stimulation implantation due to its miniature size. However, it was found to be excruciatingly difficult and time consuming to form a reliable contact between the bump style PC board interconnect and the PDMS device. Static charge and the stretchabilty of PDMS made alignment very problematic. The addition of the aliment pins did not alleviate the time consumption necessary to attach the device onto the interconnect, nor did it increase the reliability of electrical contact. The glass interconnect design was easier to interface with the PDMS devices. This was due to the elastomeric nature of PDMS that give it a sticky-like property, facilitating its adherence to glass. The PDMS devices can be bonded temporarily or permanently to the glass connector using methods described in Chapter 3 section2.4. Temporary bonding can be used to conduct electrical continuity testing to determine which devices were viable, while permanent bonding is more for long-term studies of the devices. The design dimensions of the glass interconnects were bulky for implantation purposes, but allowed it to be compatible with off the self electronic components such as edge connector and ribbon cable for external electronic interfacing. For implantation purposes the design and fabrication of the glass interconnects is scalable, however the connection to external electronics will require custom made components. With the generation of the electrical connector, more extensive studies can be pursued. The devices will be connected to a current source with stimulation biphasic pulse applied to monitor the effects on the metal traces over time. 
A $2^{\text {nd }}$ generation iteration of inspections by LLNL and implantation test by USC physicians was necessary in order to discern any remaining difficulties in fabrication, handling and surgical manipulation of the device. Only one modification was requested by the surgeon to be incorporated in the next generation devices. The suggestion is to include a thicker rib or tab at the insertion end of the device, rather than a series of small ribs, to prevent the end from lifting during tack insertion. With the added improvements, the $2^{\text {nd }}$ generation establishes a foundation for a process flow from which the final device can be fabricated. One of the $2^{\text {nd }}$ generation devices is pictured in Fig. 5.14.

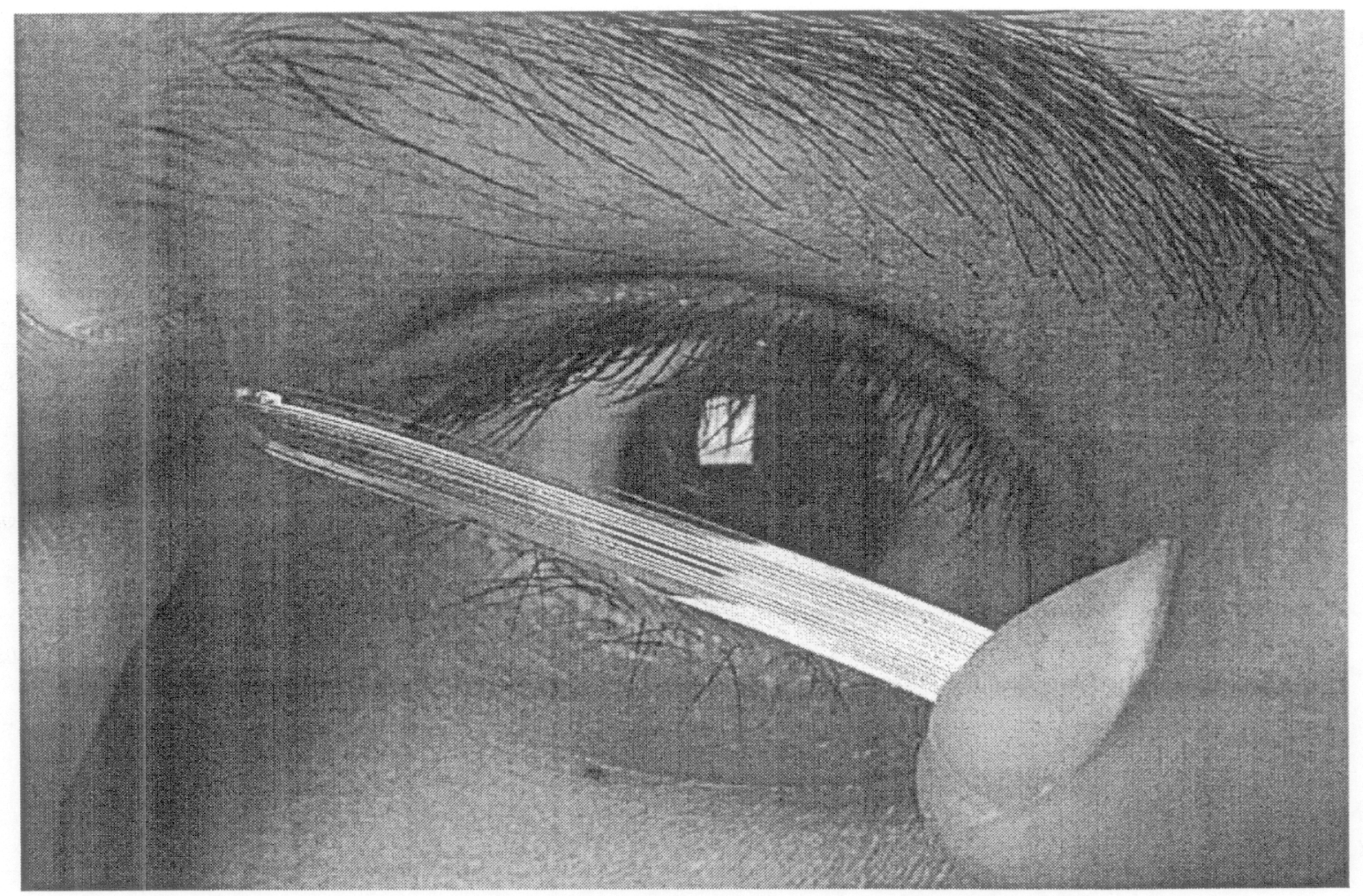

Figure 5.14. One of LLNL's stretchable microelectrode arrays held next to the eye of summer student Armando Tovar

\subsection{REFERENCE:}


[1] G. Kovacs, Micromachined Transducers Sourcebook. New York: McGraw-Hill, 1998.

[2] M. Madou, Fundamentals of Microfabrication. New York: CRC, 1997.

[3] C. A. Puliafito, "Retinal topography with optical coherence tomography," 2002.

[4] M. Humayun, "Intraocular Retinal Prosthesis," Tr. Am. Ophth. Soc, vol. 99, pp. 271-300, 2001. 


\section{CHAPTER 6: SURFACE ANALYSIS AND CURING KINETICS}

\subsection{INTRODUCTION}

This work addresses the impact of PDMS surface properties on the development of reliable fabrication processes. Surface contamination alters the surface properties of the material, affecting the repeatability of fabrication processes, adhesion of coatings, and possibly bulk properties of thin structures. In the case of PDMS, common forms of surface contamination include siloxane surfactants [1], unreactive oligomeric siloxane compounds, and PDMS prepolymer residues [2]. Such contaminates are mobile and tend to migrate to the PDMS surface causing adhesion failures. Generally these contaminants are due to inadequate curing and the impurities resulting from the polymer's manufacturing conditions. A combination of difficulties encountered during efforts to electroplate platinum electrodes through patterned PDMS holes and batch-to-batch adhesion variability of evaporated metal films motivated the investigation of how the PDMS curing state and the oxygen plasma treatment affects surface properties and processing characteristics.

Oxygen plasma treatment is used to promote wetting of the PDMS surface as well as metal adhesion. The oxidized surface also facilitates PDMS/PDMS, PDMS/ glass, and PDMS/silicon bonding. Oxygen or nitrogen plasmas are often used to activate the polymer surfaces. Oxygen plasmas tend to make the surface more acidic by forming carboxyl groups, while nitrogen treatment creates more basic surfaces due to the formation of amine groups [3]. Surface analysis tools such as time-of-flight secondary ion mass spectrometry (ToF-SIMS) and X-ray photoelectron spectroscopy (XPS) were utilized to reveal the surface composition attributed to different curing conditions and 
oxygen plasma surface treatment. The ToF-SIMS and XPS work is performed in collaboration with Art Nelson, Kuang-Jen Wu, and Cheryl Evans in the material science technology division.

Most commercially available silicone elastomer formulations contain freely diffusible uncured oligomers and other impurities such as catalysts. These components, which are driven to the surface due to both concentration gradients and surface energy, can form a surface boundary layer, making it difficult to obtain proper adhesion to the PDMS devices. A number of analytical techniques, such as solvent swelling measurements and dynamic mechanical analysis (DMA), were used to assess how the curing kinetics of PDMS affects the concentration of these freely diffusible residues. Understanding the effects of oxygen surface treatment and curing kinetics on the surface properties will aid in developing reliable and repeatable fabrication processes. Curing kinetics studies were performed under the guidance of Tom Wilson.

\subsubsection{Surface Analysis}

\subsubsection{TIME-OF-FLIGHT SECONDARY ION MASS SPECTROMETRY}

Time-of-Flight Secondary Ion Mass Spectrometry (ToF-SIMS) provides mass spectra characteristic of the top few atomic layers. In ToF-SIMS the sample is bombarded with a primary ion beam, typically formed from $\mathrm{Ar}, \mathrm{Xe}, \mathrm{Ga}$, or $\mathrm{Cs}$ ions which are accelerated to $5-25 \mathrm{kV}$ and focused on the sample surface, as seen in Fig. 6.1. The primary ions strike the sample surface and transfer energy and momentum to the sample. This results in desorption of neutral species, secondary electrons, and secondary ions. The secondary ions are mass detected and analyzed. The energy transferred to the 
sample decreases with distance away from the primary ion impact site. Close to the impact site, atoms and electrons are desorbed; farther from the site intact molecules are desorbed [4].

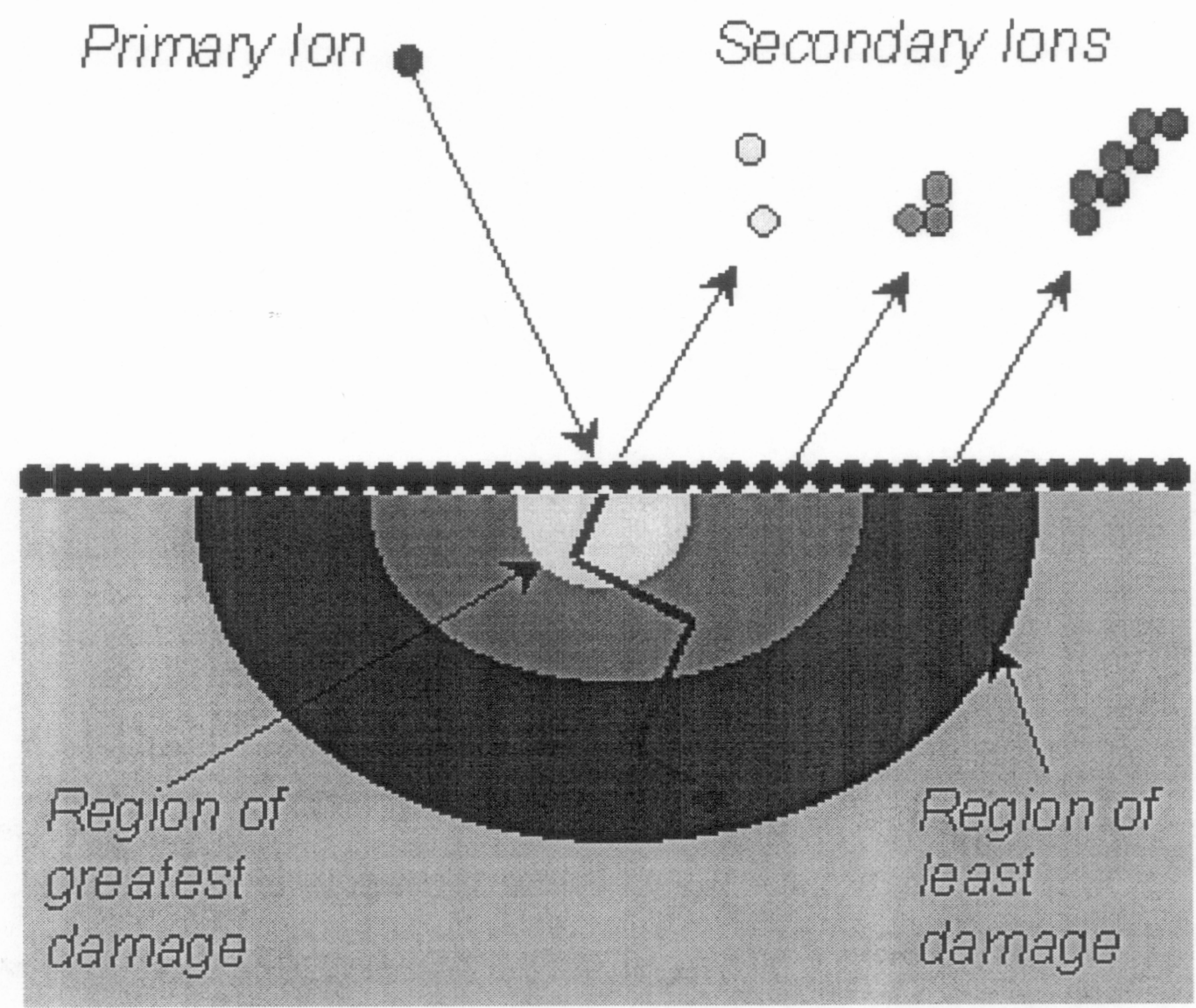

Figure 6.1. Schematic of the Time-of-Flight Secondary Ion Mass Spectrometry process. Different colors denote ionized species from sample surface.

\subsection{ToF-SIMS Measurements}

ToF-SIMS uses a pulsed primary ion beam to desorb and ionize species from a sample surface. The resulting secondary ions are accelerated into a mass spectrometer, where they are mass analyzed by measuring their time-of-flight from the sample surface to the detector. An image is generated by rastering a finely focused beam across the 
sample surface. Due to the parallel detection nature of ToF-SIMS, the entire mass spectrum is acquired from every pixel in the image. The mass spectrum and the secondary ion images are then used to determine the composition and distribution of sample surface constituents. ToF-SIMS provides spectroscopy for characterization of chemical composition, imaging for determining the distribution of chemical species, and depth profiling for thin film characterization. ToF-SIMS data is collected using a physical electronic TRIFT III time-of-flight spectrometer in conjunction with a pulsed gallium $\left({ }^{69} \mathrm{Ga}^{+}\right)$liquid-metal ion gun (LIMG) operating at $15 \mathrm{kV}, 600 \mathrm{pA}$ (high mass resolution mode) or $25 \mathrm{kV}, 60 \mathrm{pA}$ (high spatial resolution mode) is used as the primary ion source. Charge compensation is accomplished by pulsing a low energy beam onto the sample surface between each primary ion pulse.

\subsubsection{X-RAY PHOTOELECTRON SPECTROSCOPY}

The basis of XPS lies in Einstein's explanation of the photoelectric effect, whereby photons can induce electron emission from a solid provided the photons' energy (hv) is greater than the work function [5]. In XPS, a monochromatic beam of X-ray is incident upon a solid surface, causing photoemission from both core and valence levels of atoms in the near surface region into the vacuum [1], as illustrated in Fig. 6.2. The kinetic energy of the photoelectrons leaving the sample is measured, and this gives a spectrum with a series of photoelectron peaks. The binding energy associated with the peaks is characteristic of a particular element. The peak areas can be used to determine the composition of the surface. The shape of each peak and the binding energy can be 
slightly altered by the chemical state of the emitting atom [4]. The fundamental equation used in XPS is [5]:

$$
\mathbf{E}_{\mathrm{k}}=h v-\mathbf{E}_{\mathrm{B}}-\varphi
$$

Where $\mathrm{E}_{\mathrm{k}}$ is the photoelectron kinetic energy, $h v$ is the excited photon energy, $\mathrm{E}_{\mathrm{B}}$ is the electron binding energy, and $\varphi$ is the work function [5]. Analysis of peak shifts, peak areas, and appearances or disappearance of certain peaks were monitored following reactions on PDMS surfaces due to specific treatments, were monitored.

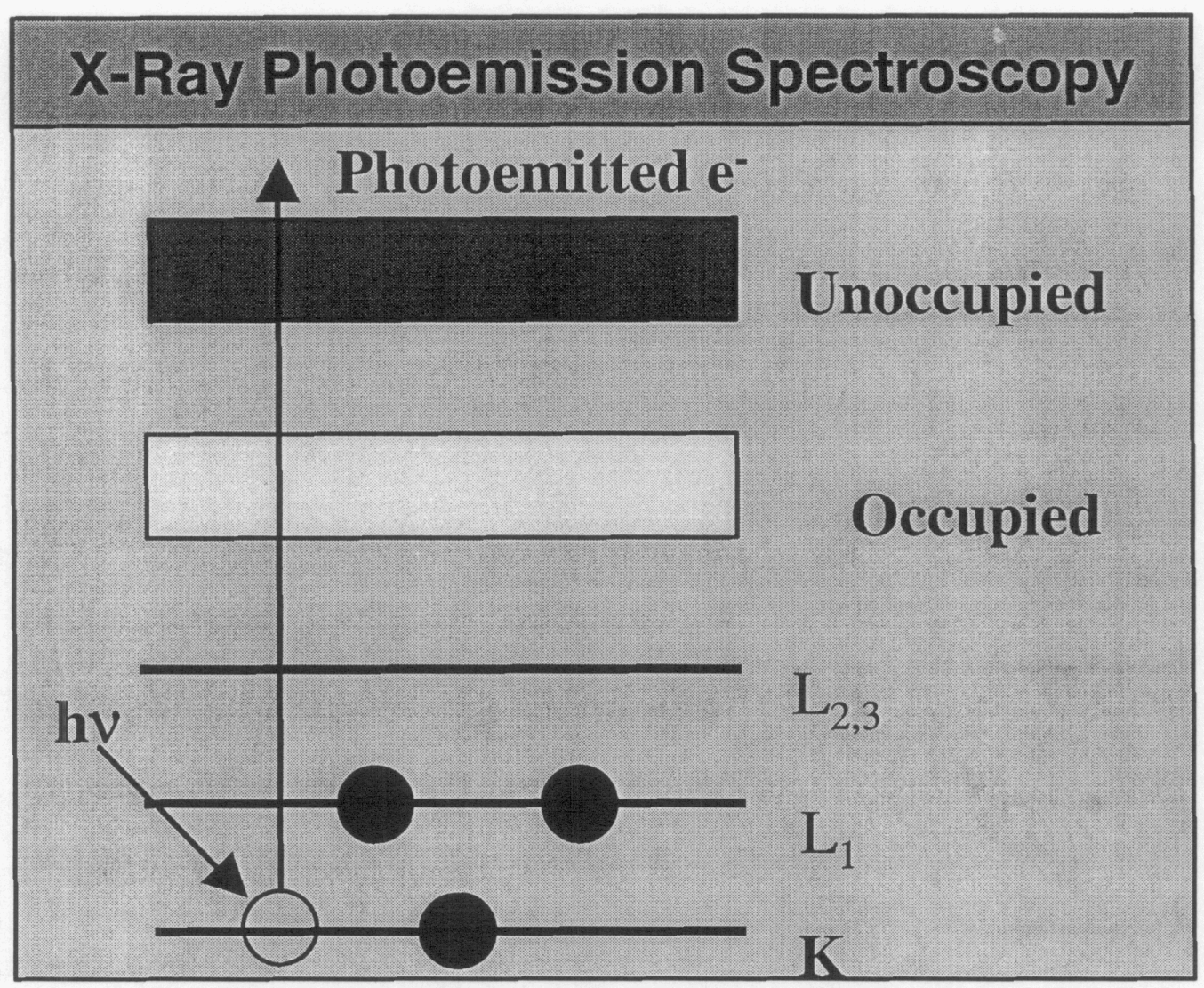

Figure 6.2. Schematic of the X-ray photoelectron spectroscopy process

\subsection{XPS Measurement}

The XPS data is collected using a Quantum 2000 scanning XPS system with a focused monochromatic $\mathrm{Al} \mathrm{Ka}$ x-ray $(1486.7 \mathrm{eV})$ source for excitation and a spherical 
section analyzer. The $\mathrm{x}$-ray beam is incident normal to the sample and the $\mathrm{x}$-ray detector is at $45^{\circ}$ away from the normal. The pass energy is $23.5 \mathrm{eV}$ giving an overall energy resolution of $0.3 \mathrm{eV}$. The collected data are referenced to an energy scale with binding energies for $\mathrm{Cu} 2 \mathrm{p}_{3 / 2}$ at $932.72 \pm 0.05 \mathrm{eV}$ and $\mathrm{Au} 4 \mathrm{f}_{7 / 2}$ at $84.01 \pm 0.05 \mathrm{eV}$. Binding energies are also referenced to the $\mathrm{C}$ 1s photoelectron line arising from adventitious carbon at $284.6 \mathrm{eV}$. Low energy electrons and argon ions are used for specimen neutralization.

\subsubsection{PDMS Cure Kinetics}

\subsubsection{SWELLING AND EXTRACTION}

During the development of the fabrication processes for PDMS metalization, inconsistent batch-to-batch metal adhesion is observed. We suspect that residual PDMS fragments migrating to the surface influence the inconsistency in metal adhesion. This speculation initiated a series of experiments aimed at understanding the relationship between curing conditions (time and temperature) and extractable components in commercial PDMS resins, which is a necessary step for developing reliable and reproducible fabrication processes. The extent of curing was determined by a combination of solvent extraction and solvent swelling of the cured PDMS. In extraction, the amount of extractable material is a function of both the degree of cure and of the percentage of contaminants which cannot be reactively coupled into the PDMS network. In solvent swelling, the degree of swelling of the cured PDMS in a good solvent can be related to the cross-link density according to the equation of Flory and Rehner [6]: 


$$
-\left[\ln \left(1-v_{2}\right)+v_{2}+\chi_{1} v_{2}^{2}\right]=V_{1} n\left[v_{2}{ }^{0.33}-v_{2} / 2\right]
$$

where $v_{2}$ is the volume fraction of polymer, $V_{1}$ is the molar volume of the solvent, $\chi_{1}$ is the Flory-Huggins polymer-solvent dimensionless interaction parameter, and $\mathrm{n}$ is number of network chains per unit volume. The cross-link density is proportional to $n$ [6]. The higher the cross-link density, the more fully reacted the PDMS is and the higher its elastic modulus will be. Both measurements provide valuable information on the cure process and on the residual materials that can migrate to the PDMS surface, compromising adhesion.

\subsubsection{DYNAMIC MECHANICAL ANALYSIS}

Dynamic Mechanical Analysis (DMA) characterizes the viscoelastic properties of materials. DMA measures the modulus and the damping properties as a sample deforms under sinusoidal shear stress. Viscoelastic behavior of a polymer means that it has both solid and liquid characteristics that change with temperature and time. To measure viscoelastic properties, a DMA applies a sinusoidal force to a sample then measures the resulting sample deformation as a function of frequency [7]. The sample is placed between parallel plate geometry fixture with a fixed base and oscillating spindle, as seen in Fig. 6.3. The sample strain response lags behind the input stress wave with respect to time and the lag is known as the phase angle [7]. The ratio of the dynamic stress to the dynamic strain provides the complex modulus that includes both the dynamic storage shear modulus, $\left(G^{\prime}\right)$ and the dynamic loss shear modulus $\left(G^{\prime \prime}\right)$. The storage modulus refers to the ability of a material to store energy and is related to the stiffness of the material. The loss modulus represents the heat dissipated by the sample as a result of 
molecular motions and reflects the damping characteristics of the material. These measurements can be used to understand the changes in stiffness of a material as a function of temperature and time. $\mathrm{G}^{\prime}$ can be directly correlated to the Young's modulus E, for an ideal rubber with a Poisson's ratio $v \sim 0.5$, using the following relationship [8]:

$$
\mathbf{E}=\mathbf{3 G}^{\prime}
$$

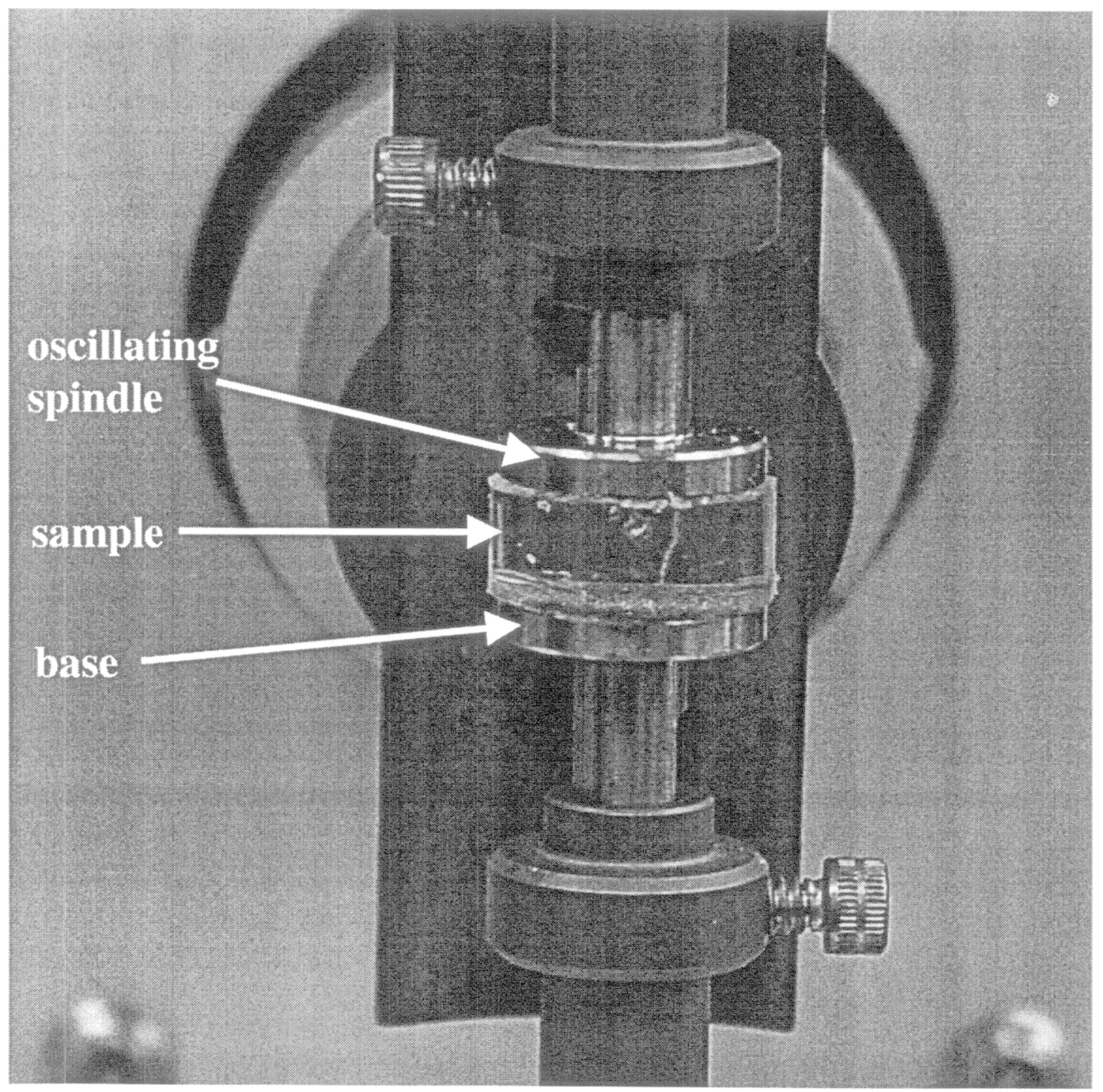

Figure 6.3. Rheometric dynamic spectrometer (model RDS-2) for dynamic mechanical analysis testing 
Additionally, in elastomeric materials $\mathrm{G}^{\prime}$ can be used to approximate the shear modulus $\mathrm{G}$, which is directly proportional to the cross-link density for an ideal elastomer [8].

\subsection{EXPERIMENTAL APPROACH}

\subsubsection{Surface Analysis}

\subsubsection{TIME DEPENDENT TOF-SIMS}

High spatial resolution Time-of-Flight Secondary Ion Mass Spectrometry (ToFSIMS) was used to examine and map the surface chemistry of exposed gold (Au) pads in several PDMS patterned holes following $1 \mathrm{hr}$. and 24hr. cures. The purpose of the exposed $\mathrm{Au}$ pads in the patterned holes served as the seed layer for platinum $(\mathrm{Pt})$ electrodeposition, which was not a successful process.

Silicon wafers were coated with gold $(\mathrm{Au})$ using Chromium $(\mathrm{Cr})$ as the adhesion layer. These wafers were then patterned with thick photoresist (AZ PLP TM100XT, Clariant). Then PDMS was Spun on the wafers and cured at $66^{\circ} \mathrm{C}$ for $1 \mathrm{hr}$. and $24 \mathrm{hrs}$. Acetone was used to remove photoresist followed by an isopropyl alcohol (IPA) rinse, exposing the Au through $300 \mu \mathrm{m}$ diameter holes in PDMS. The samples were loaded for measurement one hour following removal from the oven. The typical analysis area was $100 \mu \mathrm{m} \times 100 \mu \mathrm{m}$.

\subsubsection{EFFECTS OF OXYGEN PLASMA TREATMENT}

High spatial resolution Time-of-Flight Secondary Ion Mass Spectrometry (TOFSIMS) and X-ray photoelectron spectroscopy (XPS) were used to examine and map the surface chemistry of PDMS with and without oxygen plasma treatment. PDMS was 
photolithographically patterned on gold-coated silicon wafers and cured at $66^{\circ} \mathrm{C}$ for 24 hours (See chapter 5). The samples were cleaned with $20 \% \mathrm{HCL}$ for $8 \mathrm{~min}$. One sample was rinsed with ethanol and treated with an oxygen plasma at RF power of 100 watts and oxygen flow at $300 \mathrm{sccm}$ for $1 \mathrm{~min}$ and another sample was left untreated. The samples were then packaged in a nitrogen rich environment until loaded into the machine to preserve the freshness of the surface.

\subsubsection{Curing Kinetics}

\subsubsection{SWELLING AND EXTRACTION}

These experiments were conducted to assess the effect of cure time and temperature on the final cure state of PDMS samples by swelling and extraction in toluene. Resin samples were mixed, degassed, then 5 grams of each was placed in $70 \mathrm{~mm}$ diameter aluminum pans. Samples were cured at temperatures from $23^{\circ} \mathrm{C}$ to $150^{\circ} \mathrm{C}$, and at cure times from 15 minutes to 96 hours in gravity convection ovens. After curing, samples were removed from the aluminum pans, cut into $1 \mathrm{~cm}$ wide strips, placed in a quantity of toluene 30 times their weight and moderately agitated for 72 hours at room temperature. Following this extraction, samples were removed from the toluene and weighed. Samples were then dried for 12 hours at room temperature followed by 1 hour at $90^{\circ} \mathrm{C}$ under high vacuum and reweighed. Swell ratio was then calculated as the total weight of the swollen PDMS piece divided by the original sample weight. Soluble fraction of the PDMS was calculated as the weight of material extracted divided by the initial weight. 


\subsubsection{DYNAMIC MECHANICAL ANALYSIS}

The transient curing of Sylgard 184 was observed by dynamic mechanical analysis in order to assess whether the manufacturer suggested cure times were sufficient for complete development of mechanical properties. These experiments complement the extraction tests. Testing was carried out at constant temperatures of 60,75 , and $90^{\circ} \mathrm{C}$ using a Rheometrics Dynamic Spectrometer (model RDS-2) and a $15 \mathrm{~mm}$ parallel plate geometry with a $1.00 \mathrm{~mm}$ gap. The transient cure testing was carried out in dynamic oscillatory mode at a frequency of 6.28 radians/second and at shear strain amplitudes from 0.1 to $50 \%$, adjusted during testing to stay within the linear viscoelastic limit as determined previously through isothermal strain sweep measurements. Testing proceeded by loading sample into the test geometry, setting the sample thickness, and then heating the sample quickly to the test temperature. Data collection started immediately after sample loading and was taken at 10 to 30 second intervals depending on temperature. Generally, it took less than 3 minutes after loading for the sample to approach the test temperature. From the raw strain and torque data, the dynamic storage (elastic) shear modulus $G^{\prime}$ and the dynamic loss (viscous) shear modulus $G^{\prime \prime}$ were calculated by the instrument software (Rheometrics Orchestrator) using well-known relations [9].

\subsection{RESULTS}

\subsubsection{Surface Analysis}

\subsubsection{TIME DEPENDENT TOF-SIMS ANALYSIS}




\subsection{ToF-SIMS analysis following $1 \mathrm{hr}$. cure}

One-hour cure PDMS holes were examined with ToF-SIMS, revealing the presence of siloxane on the patterned gold pad, which suggested incomplete curing of the PDMS. The characteristic fragmentation patterns at $\mathrm{m} / \mathrm{z}=28(\mathrm{Si}), 43\left(\mathrm{SiCH}_{3}\right)$ and 73 $\left(\mathrm{Si}\left(\mathrm{CH}_{3}\right)_{3}\right), 147\left(\mathrm{Si}_{2} \mathrm{O}\left(\mathrm{CH}_{3}\right)_{5}\right)$ and $207\left(\mathrm{Si}_{3} \mathrm{O}_{3}\left(\mathrm{CH}_{3}\right)_{5}\right)$ represent the typical fingerprint of PDMS, Fig. 6.4a. Positive and negative SIMS survey spectra were acquired for each hole. The peaks at 73 AMU and 147 AMU indicate residual PDMS in the holes.

The surface of the PDMS was also examined with ToF-SIMS following the one-hour cure. In addition to the finger print spectrum of PDMS, photoresist residuals were found on the surface indicating photoresist out gassing, Fig. $6.4 \mathrm{~b}$.

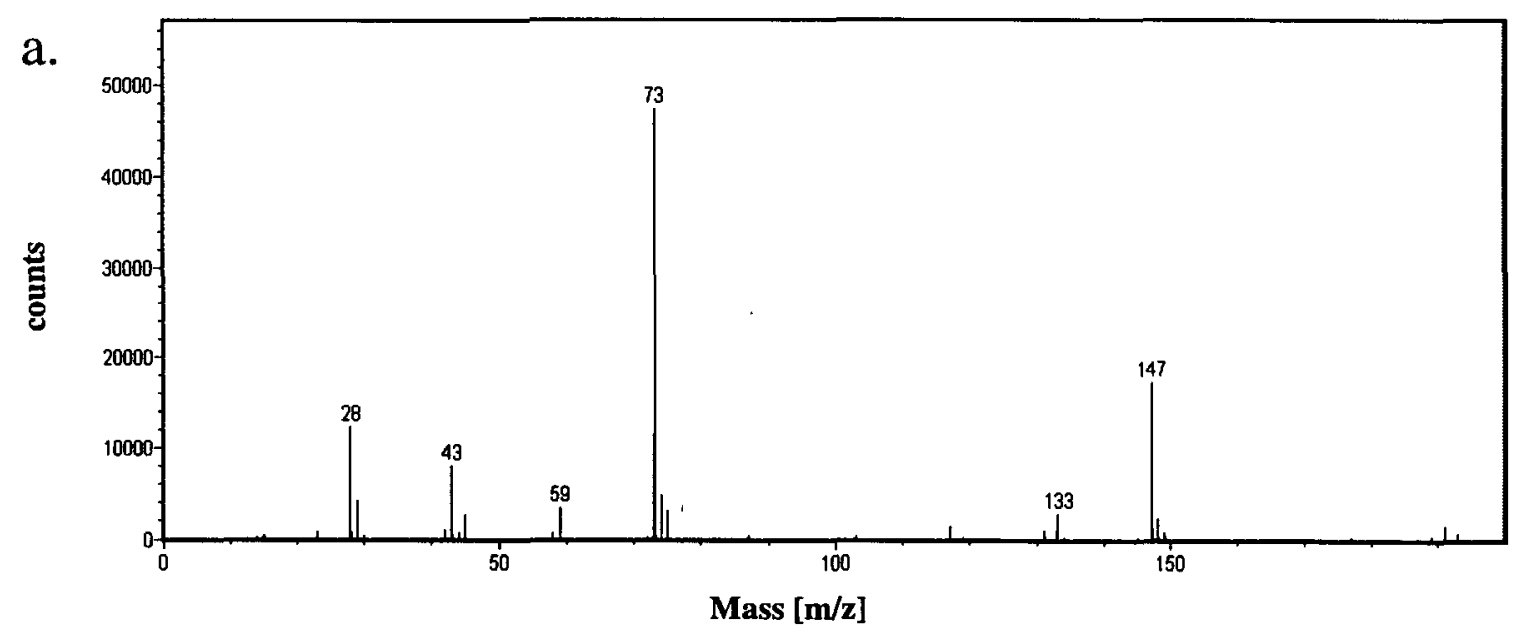




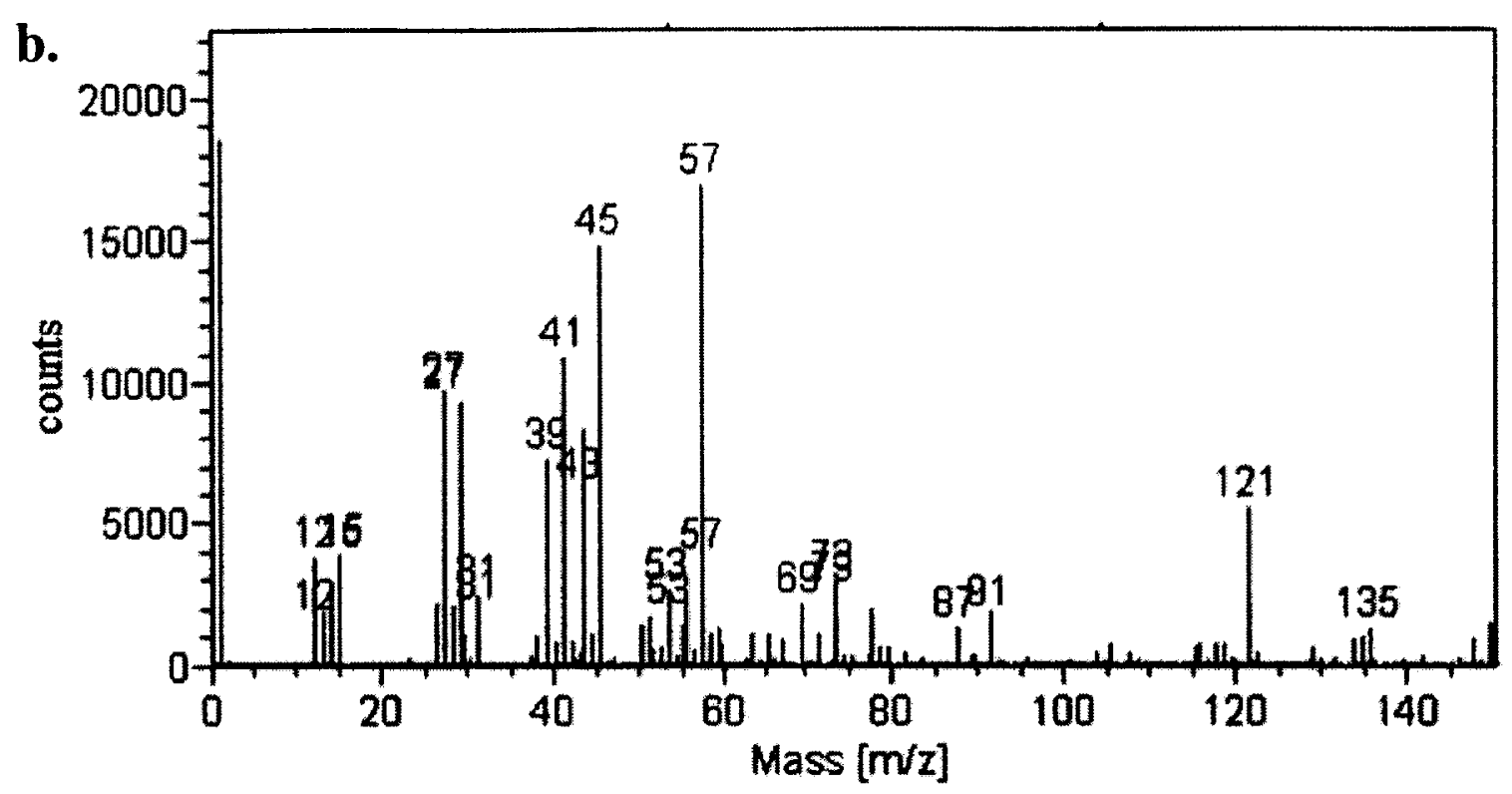

Figure 6.4. ToF-SIMS spectra of characteristic PDMS surface b) ToF-SIMS spectra of PDMS surface with patterned holes following thour cure

\subsection{ToF-SIMS Analysis following 24 hour cure}

ToF-SIMS analysis was conducted on 24 hour cured PDMS in the region of the patterned holes to determine the surface species present. The measurement was first conducted on the freshly prepared sample one hour following the 24-hour curing process; the spectrum is shown in Fig. 6.6a. The second measurement was conducted on the same sample after it was stored under vacuum for 24 hour; the spectrum is shown in Fig. 6.6b. The characteristic PDMS fragment intensity at $\mathrm{m} / \mathrm{z}=28,43,73$ and 147 increased significantly after the sample was left under vacuum for 24 hours. Table 6.1 summarizes the peak intensity of PDMS fragments inside the patterned holes for fresh 24-hour cure samples and for 24 hour cure samples that have been under vacuum for 24 hours. A sample with only gold film, with chromium adhesion layer, was used as a control with no spun PDMS and no PDMS peaks were detected. 


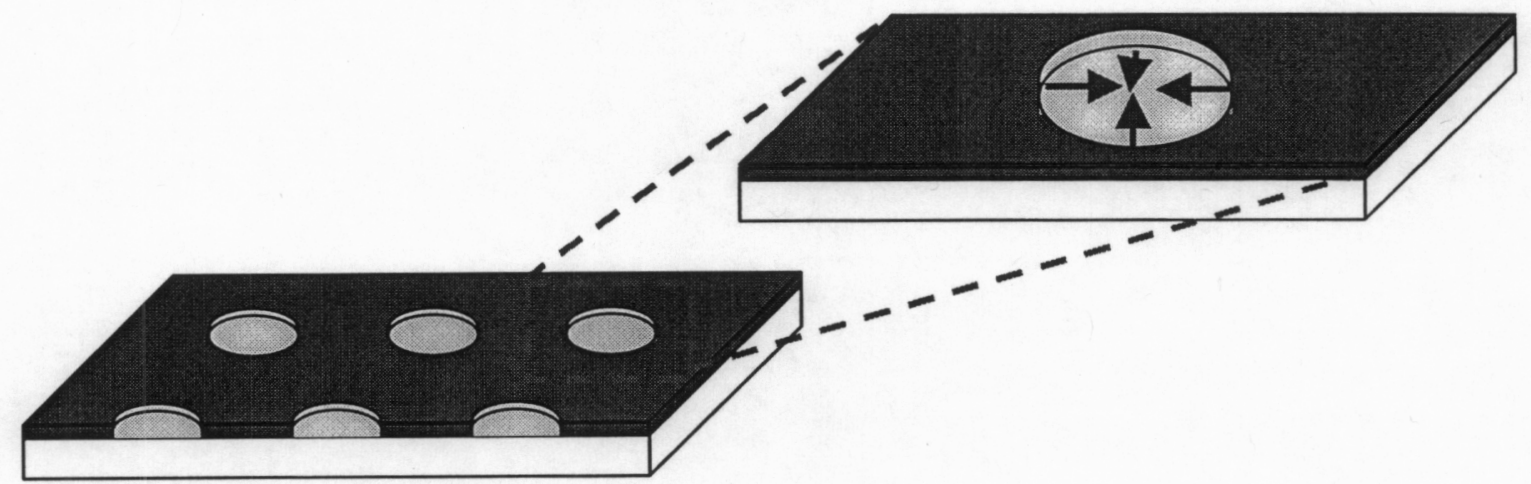

Figure 6.5. Patterned PDMS film on gold-coated silicon wafer. Close-up illustrating PDMS migration by diffusion.

Table 6.1. PDMS peak intensity inside patterned hole using ToF-SIMS analysis

\begin{tabular}{|l|l|l|l|l|}
\hline Sample & $\mathbf{m} / \mathbf{z}=\mathbf{2 8}$ & $\mathbf{m} / \mathbf{z}=\mathbf{4 3}$ & $\mathbf{m} / \mathbf{z}=\mathbf{7 3}$ & $\mathbf{m} / \mathbf{z}=\mathbf{1 4 7}$ \\
\hline Fresh $^{1}$ & 33408 & 19653 & 74455 & 18463 \\
\hline 24 hr vaccum $^{2}$ & 180631 & 119819 & 430623 & 221931 \\
\hline
\end{tabular}

'Fresh: 24 hour cured sample measured 1 hour after removal for the oven

${ }^{2} 24$ hr vacuum: 24 hour cured sample measured after 24 -hour storage under vacuum. 

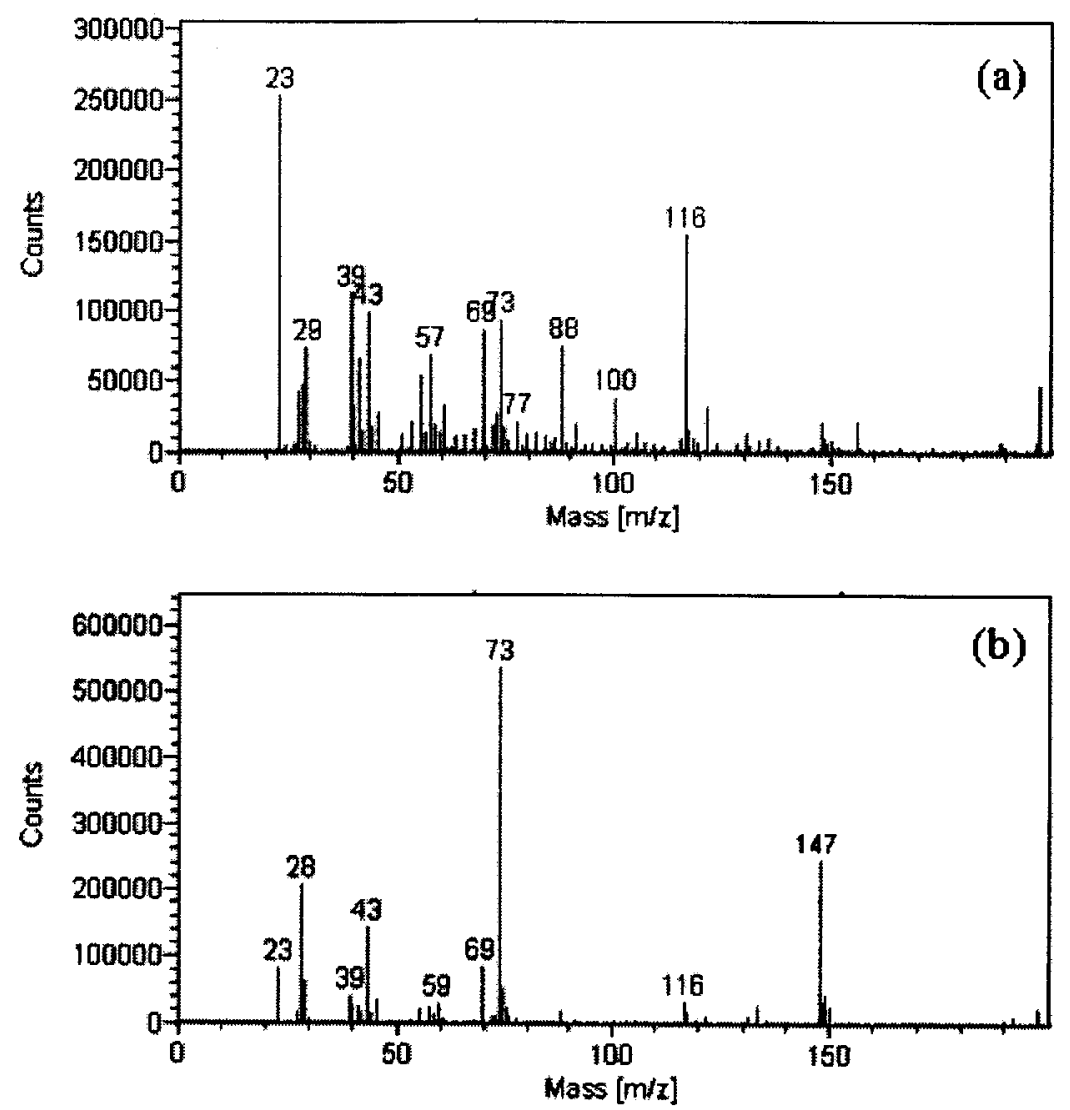


\section{CHAPTER 7: MECHANICAL TESTING}

\subsection{INTRODUCTION}

It is essential that the metal traces on the PDMS film be robust, as stretching and bending occur during fabrication and implantation of the devices. Therefore it is imperative to understand how much stress can be tolerated by the devices before failure and how to maximize the stretchablity of these devices. During the PDMS metalization process it is observed that a combination of fabrication variables induce wrinkling of the metal traces on the PDMS film as seen in Fig. 7.1. From preliminary uniaxial manual tensile tests, these wrinkles are thought to augment the stretchablity of the metal traces with average strains of $7 \%(\sigma=0.01)$ [1]. These results prompted further investigation of the mechanical properties of these metal traces on PDMS. In order to optimize robustness and stretchablity of the metal traces it is vital to understand the stresses causing these wrinkles.

There are two primary categories of stresses exerted on the devices: applied stresses and residual stresses. Applied stresses are external stresses that result from stretching during handling, surgical implantation, and conforming to the retina. Residual stresses often govern thin film behavior [2]. These stresses are divided into two categories: extrinsic and intrinsic stresses. Extrinsic residual stress is caused by external conditions such as thermal gradients or mismatches in coefficient of thermal expansion $[2,3]$. Intrinsic stress is related to the microstructure created in the film as the atoms are deposited onto the substrate [2].

This chapter examines the tolerance of the devices to applied external stresses and how that tolerance is affected by the intrinsic residual stresses in both the PDMS and 
metal film. First, this chapter measures the stretchablity of the metal traces using both automated and manual uniaxial tensile testing instruments. The tensile test work is performed in collaboration with Christina Park for her masters thesis at the Massachusetts Institute of Technology [4]. The second topic of this chapter, examines how processing induced residual stresses influence the stretchablity of the metal traces. Two residual stress issues are addressed in this chapter. One looks at the intrinsic residual stress in the PDMS films due to deposition and curing on a ridged gold-coated silicon substrate. The other examines the intrinsic residual stress issue resulting from the deposition of the metal film on to the PDMS film.

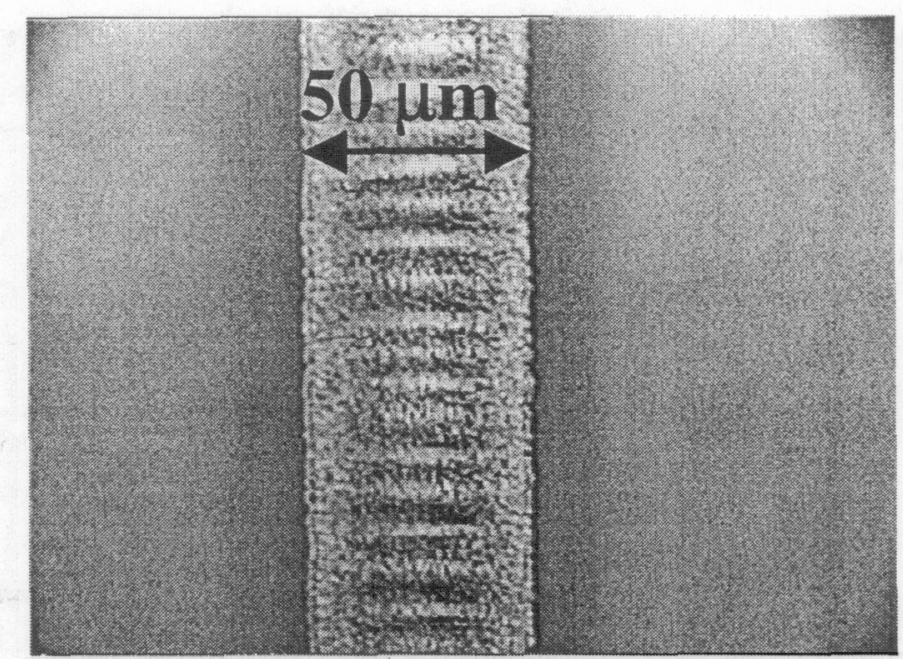

Figure 7.1. Gold traces patterned onto PDMS using evaporative deposition. The wrinkles are due to compressive stress in the film.

\subsection{THEORY}

\subsubsection{Stresses Necessary to Withstand Curvature}

While it is difficult to predict the forces that occur due to handling of the device, a simple $2 \mathrm{D}$ calculation of the forces necessary to change the conformation of the device to the curvature of the eye was performed. The radius of the eye is approximately $12 \mathrm{~mm}$ 
[5], which is the radius of curvature that must be matched by the microelectrode arrays in order to ensure uniform contact between the individual electrodes and the ganglion cells in the retinal tissue. Bending the array in this manner causes a strain at the surface of the device as described in Eqn. 7.1 [6].

$$
\varepsilon_{x}=\varepsilon_{y}=-\frac{z}{\rho}
$$

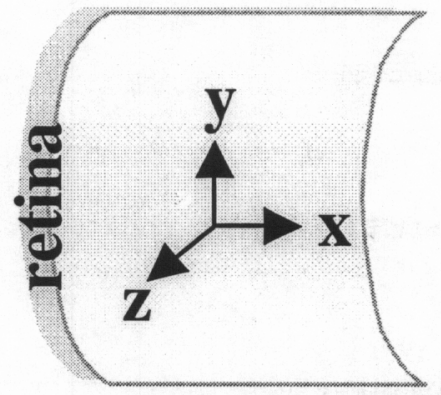

Figure 7.2. Reference axis on the retina with $\mathrm{z}$ being normal to the surface

The strains $\varepsilon_{\mathrm{x}}$ and $\varepsilon_{\mathrm{y}}$ are equal because the curvature for the square electrode array must be equivalent to the radius of the eye in both directions, Fig. 7.2. Using a $25 \mu \mathrm{m}$ thick metalized PMDS layer, Eqn. 7.1 predicts strains at the surface to approximately be $0.1 \%$ for a flat and initially unstressed gold film. This is assuming that there is no second PDMS passivation layer. Using a value of $53 \mathrm{GPa}$ for Young's modulus ,E, of the gold film [7], the stress generated in the film when the electrode array was curved was approximately $53 \mathrm{MPa}$. This was calculated using Hooke's Law, see Eqn. 72 [6]. This is approximately the same as the $55 \mathrm{MPa}$ yield stress of the gold [7].

$$
\sigma=\varepsilon \mathrm{E}
$$

If the gold films used to create the conducting traces on the PDMS surface were perfectly flat and initially unstressed before curvature, they may yield when conforming to the retina, and could potentially fail. However, as will be seen in section 7.3.2, residual 
stresses in the PDMS put the gold traces into a state of compression (Fig. 7.1) in the uncurved configuration, allowing stretching beyond the yield strain of gold.

\subsection{EXPERIMENTAL APPROACH}

\subsubsection{Applied Stresses}

Uniaxial tensile testing was performed to determine how well the devices hold up to external forces. Such forces are applied when grasping and pulling the device, using forceps, into the eye during implantation.

\subsubsection{SAMPLE PREPARATION FOR TENSILE TESTING}

A mask was designed for fabrication of the mechanical testing samples. The design variables were lead width (100 and $200 \mu \mathrm{m})$ and shape (straight and serpentine), as seen in Fig. 7.3. Each sample was composed of a single metal trace loop on a $4 \mathrm{~mm} \mathrm{x}$ $40 \mathrm{~mm}$ PDMS film. The deposition rate was kept constant at $0.2 \mathrm{~nm} / \mathrm{sec}$. The PDMS used was Sylgard ${ }^{\bigotimes} 184$ from Dow Corning (see chapter 3 section 3.2.1). The metal traces were deposited using the standard PDMS metalization process (see chapter 3. section 3.2.6). The fabrication variables that were examined included thickness of the titanium adhesion layer (5 and $50 \mathrm{~nm})$, thickness of the gold $(100,200,300 \mathrm{~nm})$ and thickness of the PDMS (40 and $70 \mu \mathrm{m})$. 


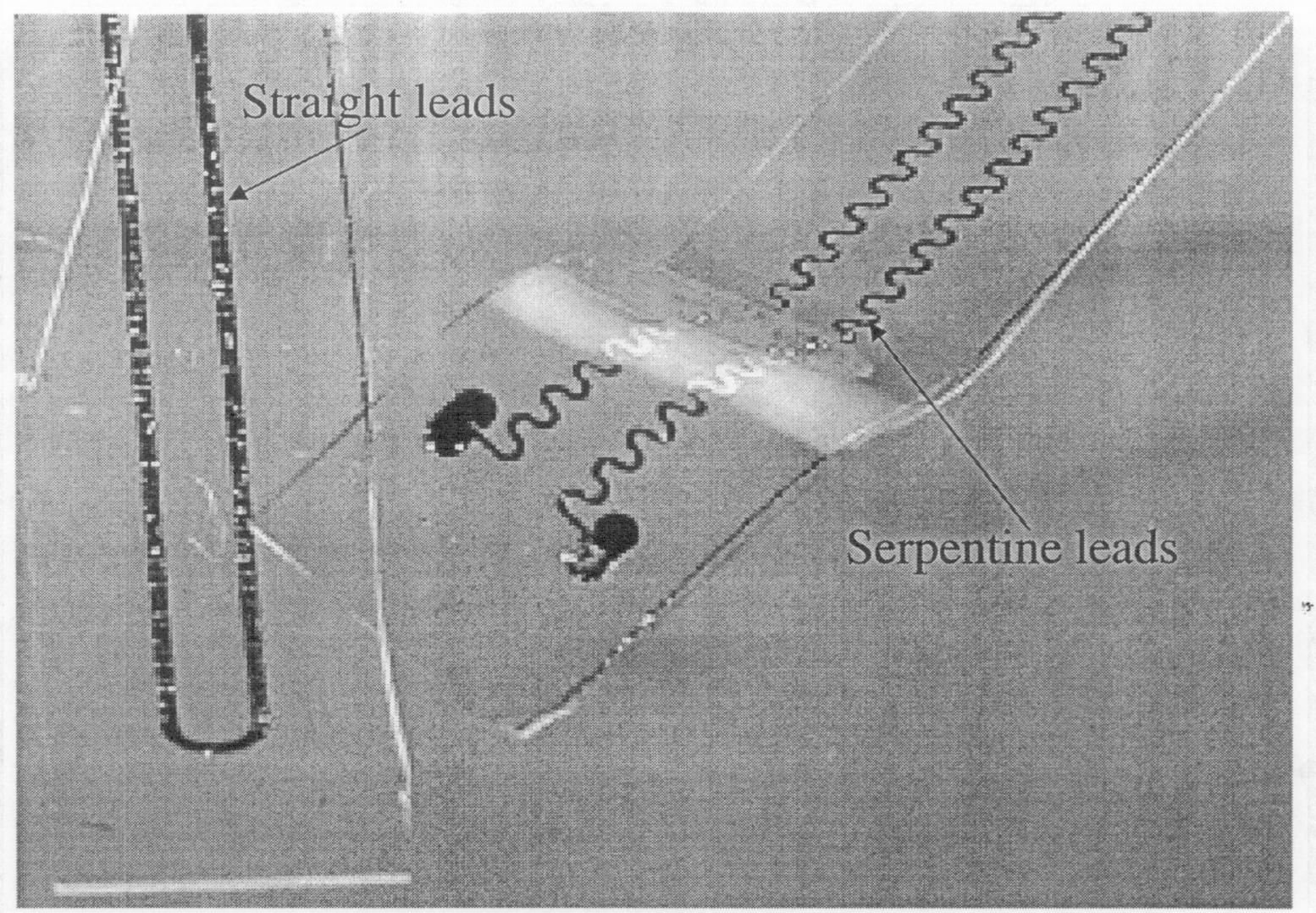

Figure 7.3. Straight and serpentine leads tested

\subsubsection{UNIAXIAL INSTRON TENSILE TESTING}

An Instron tensile tester (model 4201) with real-time visualization and monitoring was set-up to perform continuous testing of the metalized PDMS samples. The Instron was interfaced with a digital multiplexor (Agilent 34970A data acquisition/switch unit) to output measurements corresponding to position, load and resistance. This data was represented graphically using Agilent's Benchlink Data Logger. A compliant 4.5-kg. compressive load cell for testing polymers was used. The sample was loaded into the Instron as shown in Fig. 7.4. Electrical contact to the metal pads was made by aligning the device to a selectively gold-coated glass slide. Before the device was loaded into the instrument it is sandwiched between another glass slide. An extensive description of the 
Instron setup, calibration and sample loading can be found in greater detail in Park's thesis [4].
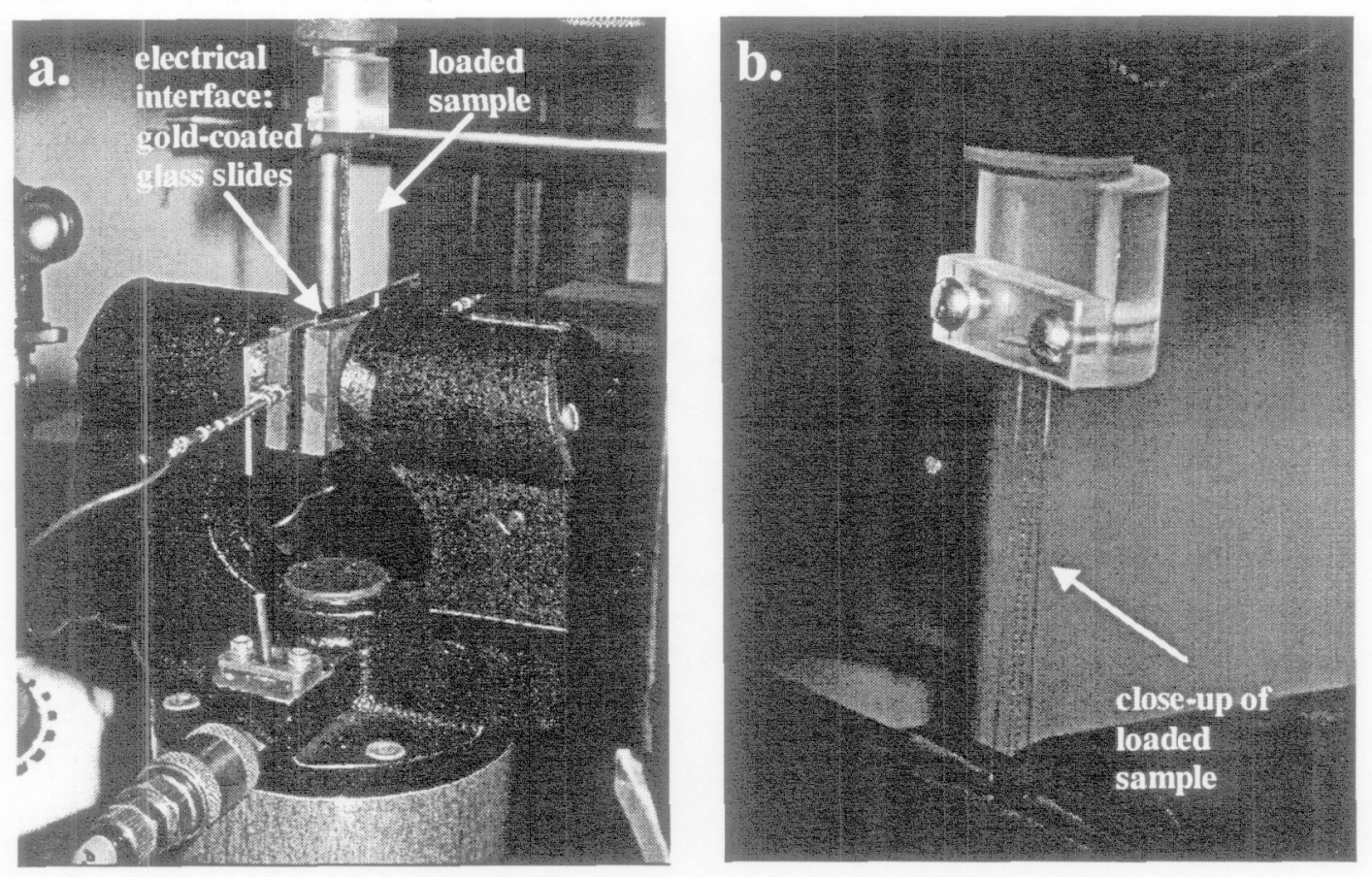

Figure 7.4. a) Instron set-up with sample loaded, b) Close-up of the loaded sample

\subsubsection{UNIAXIAL MANUAL TENSILE TESTING}

Preliminary uniaxial stretch tests presented in [1] were performed on 25 resistive heaters $(10 \mathrm{~mm} \times 15 \mathrm{~mm} \times 0.07 \mathrm{~mm})$ from the same wafer. Additional stretch tests were perfumed on 27 new samples designed for performing mechanical testing. A micrometer was used to measure axial elongation, and resistance of the metal trace was monitored as the sample was incrementally stretched, as seen in Fig. 7.5, with failure corresponding to an open circuit. Each sample was loaded into the instrument by gripping one end onto the stationary stage and the other end to a movable stage. A micrometer head was used to advance the movable stage, stretching the sample. The 
PDMS was gripped just before the metal leads and an ohmmeter was used to monitor continuity as shown in Fig. 7.5.

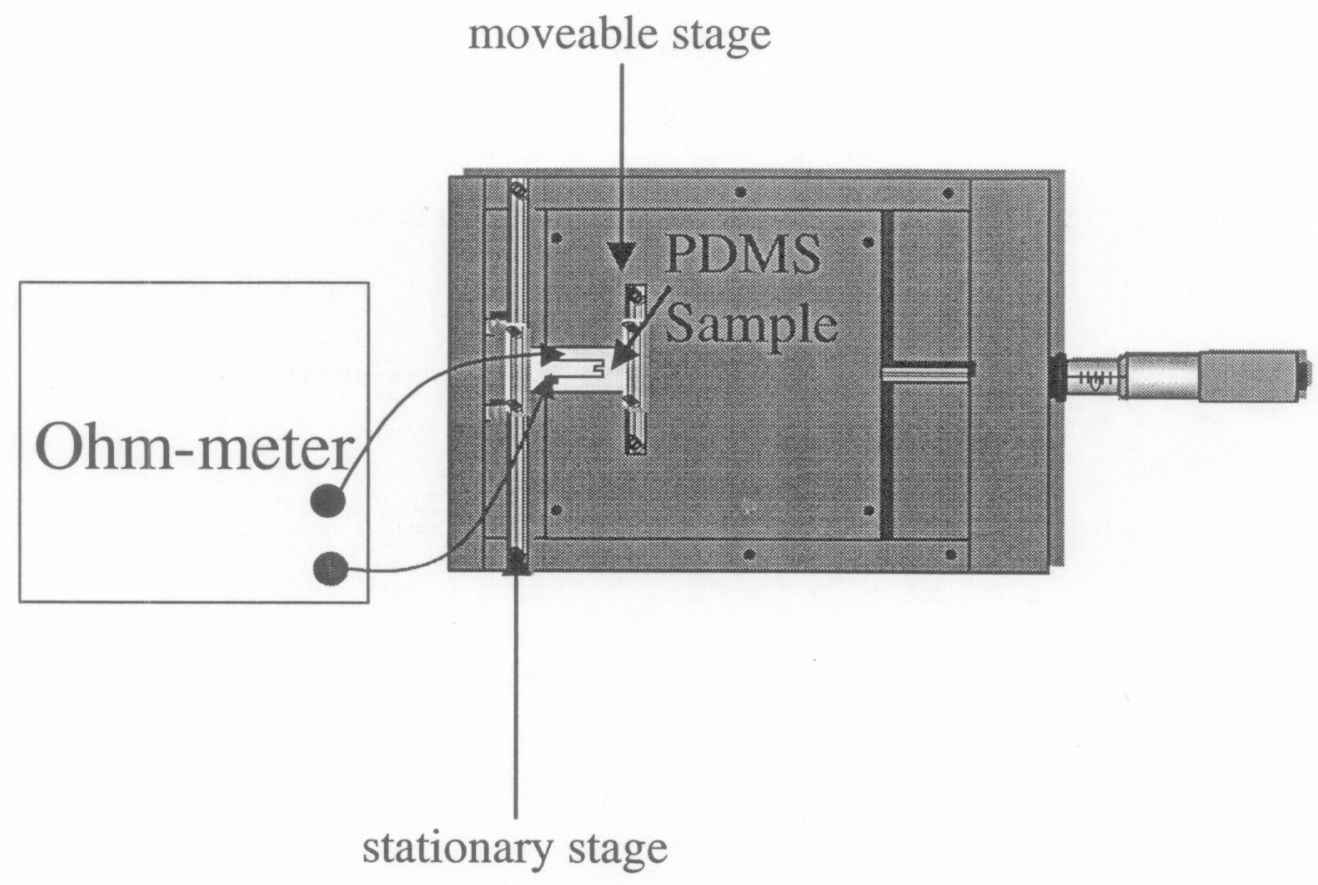

Figure 7.5. Illustration of the manual strain measurement set-up used to test the metalized traces on the PDMS.

\subsubsection{Residual Stress}

\subsubsection{PDMS FILM ON SILICON SUBSTRATE}

A $47 \mu \mathrm{m}$ thick film of PDMS was spun onto a gold-coated double-side polished silicon wafer with a thickness of $500 \mu \mathrm{m}$. A Tencor FLX-2320 Thin Film Stress Measurement instrument was then used to monitor curvature of the silicon substrate over time as the PDMS cured for 1 hour at $60^{\circ} \mathrm{C}$ and at room temperature after curing. The instrument was first calibrated using the gold-coated double-side polished silicon wafer with no PDMS film. Both sides of the wafer were coated with a gold film to eliminate thermal expansion effects due to the gold under-layer. The Tencor curvature instrument 
measures substrate deflection in a line scan across a diameter of the wafer. These measurements were then used to calculate film stresses [2]. Residual stress was calculated using the modified Stoney equation $[3,8,9]$ :

$$
\sigma_{P D M S}=\frac{1}{6}\left(\frac{E_{\text {substrate }}}{1-V_{\text {subsrate }}}\right)\left(\frac{t_{s u b s r r a t e}^{2}}{t_{P D M S}}\right)\left(\frac{1}{\rho_{f}}-\frac{1}{\rho_{s}}\right)
$$

Where $\sigma_{\mathrm{PDMS}}$ is the stress in the PDMS (positive for tensile), $\mathrm{E}_{\text {substrate }}$ is Young's modulus of the substrate, $t_{\text {substrate }}$ is substrate thickness, $t_{\text {PDMS }}$ is film thickness, $v_{\text {substrate }}$ is the Poisson's ratio of the substrate, and $\rho_{\mathrm{s}}$ and $\rho_{\mathrm{f}}$ are the measured wafer radii at the start of and during the PDMS cure. The modified Stoney equation, Eqn. 7.3, gives the average film stress and makes the following assumptions: (1) the PDMS film thickness is much less than the silicon substrate thickness; (2) the PDMS film is uniform ;(3) the stress is homogeneous over the entire wafer; (4) the film stress is constant through the thickness of the film [2]. While the PDMS film is thicker than films for which the Stoney equation is usually valid, the ratio of the modulus of PDMS to silicon is very small $\left(\mathrm{E}_{\mathrm{film}} / \mathrm{E}_{\text {substrate }}=\right.$ $10^{-4}$ ) and the deviation from the thick film relation is very small as seen in Fig. 7.6, a plot of the ratio of stresses calculated using the Stoney equation $\left(\sigma_{\text {thin }}\right)$ and Timoshenko's equation $\left(\sigma_{\text {thick }}\right)[9,10]$ for bimetallic strips. For the present example, the ratio of PDMS film to silicon substrate thickness is approximately 0.1 and the thin film stress equation over estimates the stress by $10 \%$ [3]. 


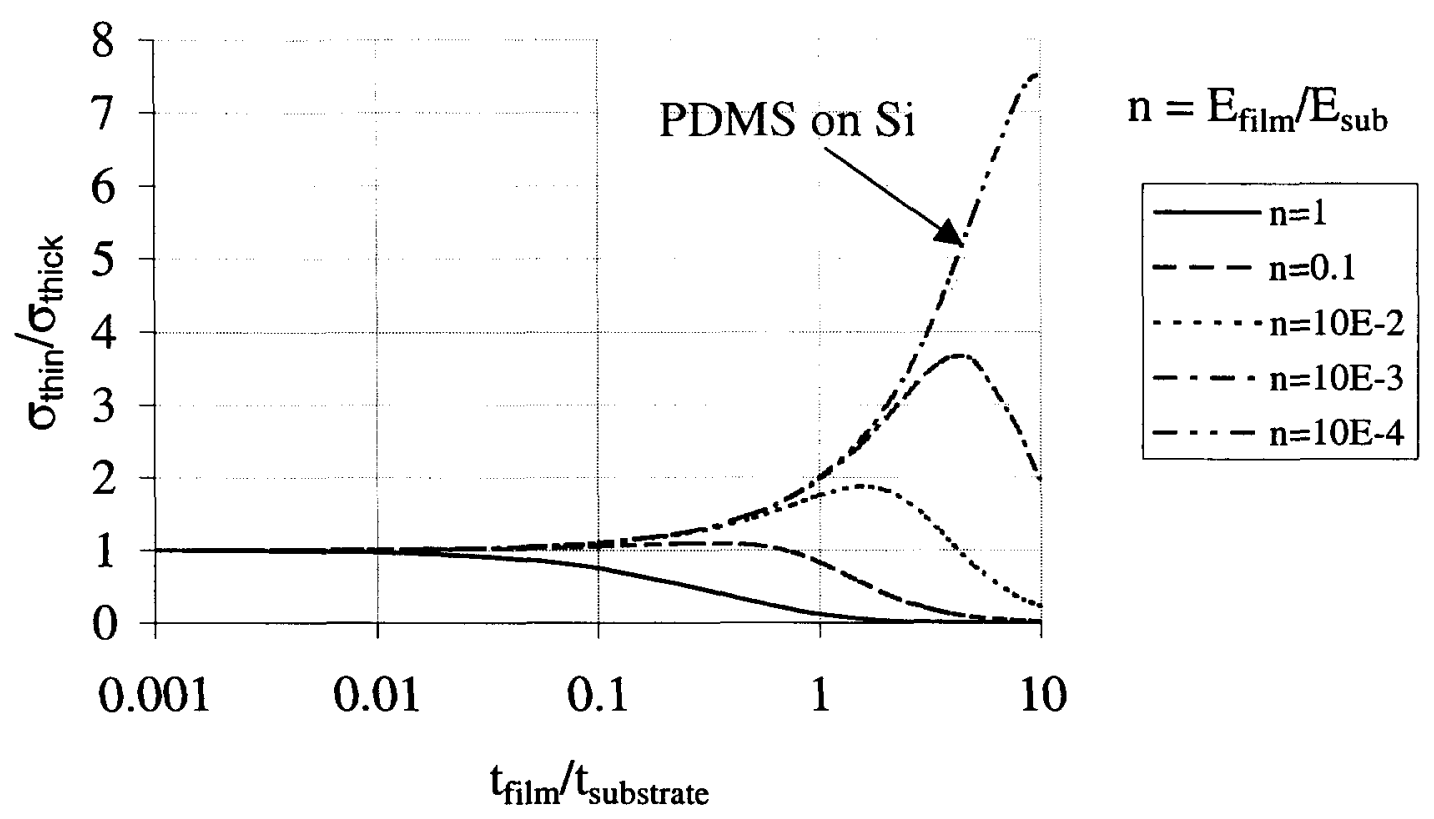

Figure 7.6. Residual stress ratio as a function of thickness ratio calculated using the Stoney equation $\left(\sigma_{\text {thin }}\right)$ and Timoshenko's equation $\left(\sigma_{\text {thick }}\right)$ for bimetallic strips.

\subsubsection{METAL FILM ON PDMS FILM}

In experiment one, metal traces were deposited onto the PDMS film using e-beam evaporation. In this case the system parameters, such as temperature and pressure, of ebeam system used could not be monitored or modified. However, metal thickness and deposition rate were varied. Qualitative visual comparison experiments were performed on 4 wafers with constant PDMS thickness $(50 \mu \mathrm{m})$ and gold thickness $(100 \mathrm{~nm})$ and variable Ti thickness $(5 \mathrm{~nm}$ and $50 \mathrm{~nm})$ and deposition rate $(0.1 \mathrm{~nm} / \mathrm{sec}$ and $0.5 \mathrm{~nm} / \mathrm{sec})$. Experiment two was performed in collaboration with Tom Beat from LLNL's Vapor Processing Laboratory in a temperature and pressure controlled sputtering system. This experiment allowed all variables to be held constant while comparing gold (500 nm) versus platinum $(500 \mathrm{~nm})$ depositions. This experiment was performed in order to 
determine if extrinsic residual stress significantly contributed to how the metal behaved on the PDMS surface. Based on the differences in coefficient of thermal expansion between the metals and the PDMS. Titanium $(20 \mathrm{~nm})$ was the adhesion layer for both samples. The metal in all the samples was deposited on the entire PDMS film with no patterning.

\subsection{RESULTS}

\subsubsection{Applied Stresses}

Upon testing, it was found that most of the samples fabricated with $50 \mathrm{~nm}$ of Ti failed prior to stretching. Upon closer inspection using a scanning electron microscope (SEM), severe cracking in the film was observed (Fig. 7.7). Therefore only the samples with $5 \mathrm{~nm}$ of Ti were used for tensile testing. Variations of PDMS and gold thicknesses did not seem to have a significant impact on the stretchablity of the metal traces. Therefore those results were averaged and the only variables compared were lead width and shape. Using the Instron tensile tester, 36 of the $100 \mu \mathrm{m}$ straight devices, 36 of the $200 \mu \mathrm{m}$ straight devices, and 24 of the $100 \mu \mathrm{m}$ serpentine devices were tested and compared. For the manual tensile tester 18 of the $100 \mu \mathrm{m}$ straight devices, 18 of the 200 $\mu \mathrm{m}$ straight devices, and 12 of the $100 \mu \mathrm{m}$ serpentine devices were tested and compared.

\subsubsection{UNIAXIAL INSTRON TENSILE TESTING}

The Instron test gave average strains to failure of $2.3 \%$ for $100 \mu \mathrm{m}$ straight leads, $2.4 \%$ for $200 \mu \mathrm{m}$ straight leads, and $4.1 \%$ for $100 \mu \mathrm{m}$ serpentine leads. The results from all of the Instron tensile testing data averaged to $2.9 \%$ strain to failure. Even after 
stretching to failure, the metal leads eventually reestablished continuity when the load was released, due to the viscoelastic nature of PDMS. As the polymer relaxes the cracked segments are brought back together, forming an ohmic contact.

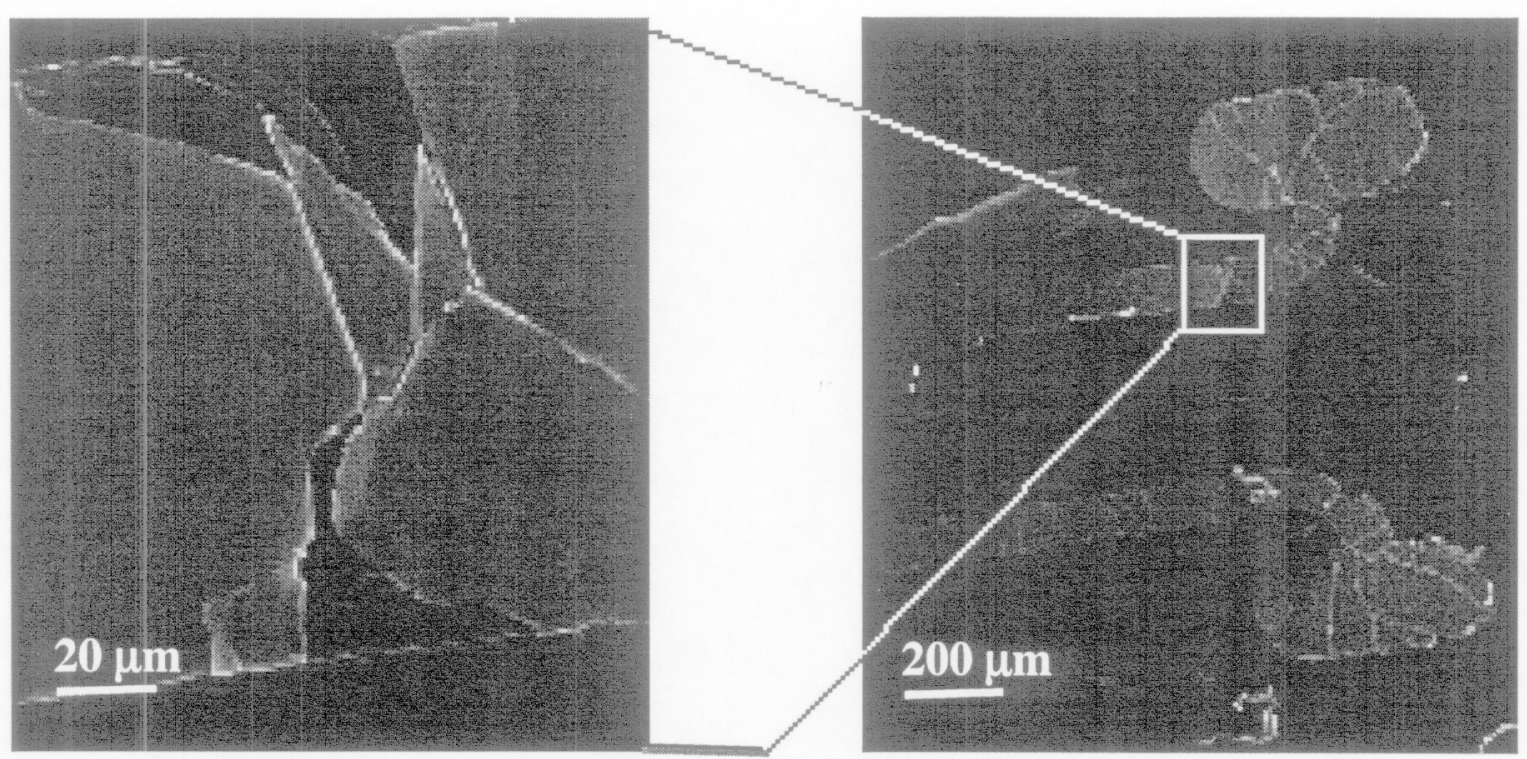

Figure 7.7. SEM image of metal trace using $50 \mathrm{~nm}$ of Ti for adhesion layer. Severe cracking can be seen. 


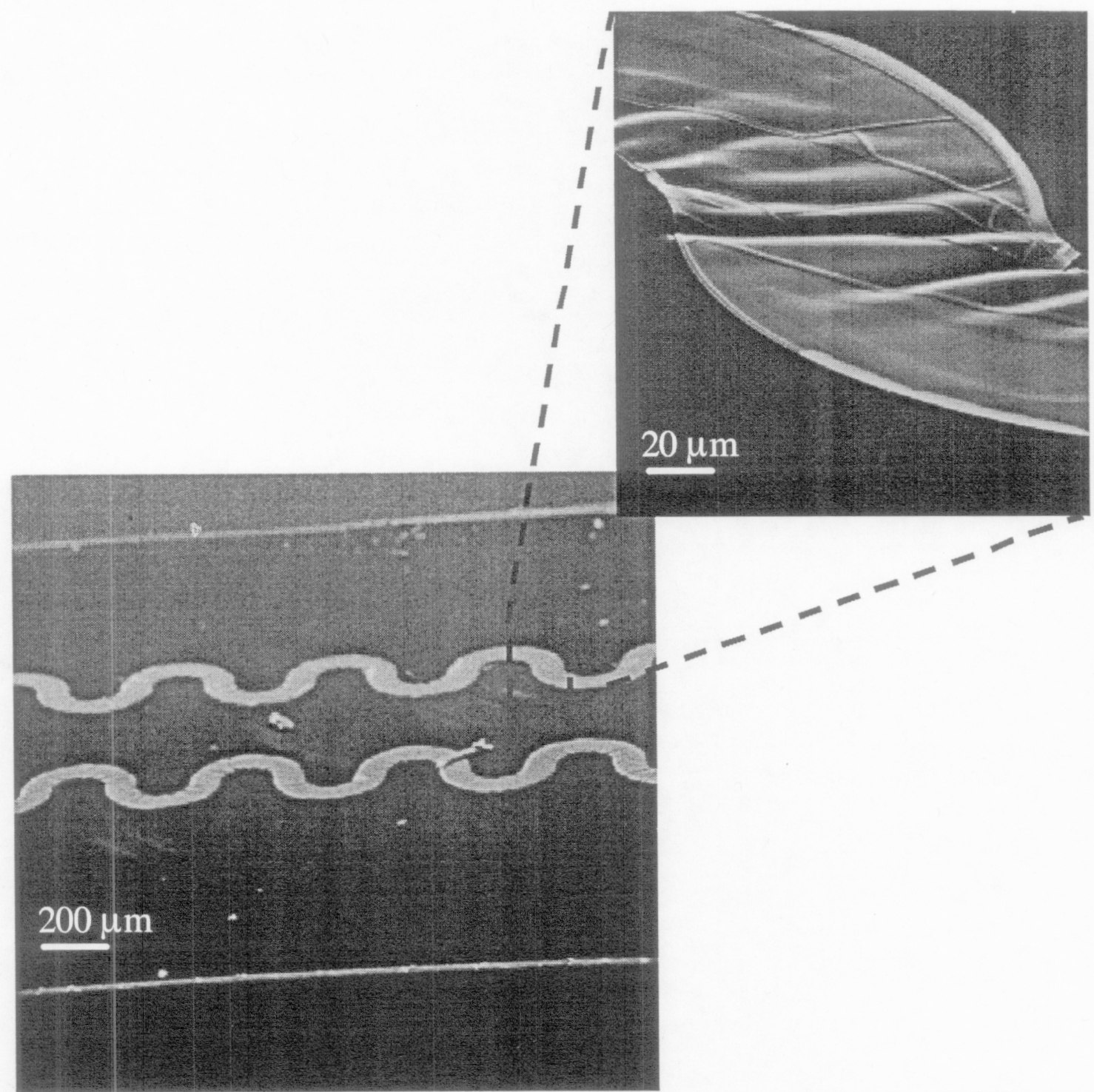

Figure 7.8. SEM of serpentine leads formed of $5 \mathrm{~nm} \mathrm{Ti/100} \mathrm{nm} \mathrm{Au} \mathrm{with} \mathrm{close-up}$ showing the wrinkles in the metal traces.

\subsubsection{UNIAXIAL MANUAL TENSILE TESTING}

The mechanical test samples for the $100 \mu \mathrm{m}$ straight leads failed at an average strain of $5.1 \%$, the $200 \mu \mathrm{m}$ straight leads failed at an average strain of $6.0 \%$, and the 100 $\mu \mathrm{m}$ serpentine leads failed at an average strain of $8.9 \%$. The results from manual tensile 
testing data averaged to $6.7 \%$ strain to failure. Even with the manual tensile testing the metal leads eventually reestablished continuity when the load was released.

\subsubsection{Residual Stress}

\subsubsection{PDMS FILM ON RIGID SUBSTRATE}

From Fig. 7.9, which shows the stress that evolves in the PDMS film during curing, the average final stress in the PDMS was $0.15 \mathrm{MPa}$, corresponding to a biaxial residual strain of $10 \%$ as calculated from the relation shown in Eqn. 7.4 [11].

$$
\sigma_{P D M S}=\varepsilon\left(\frac{E_{P D M S}}{1-v_{P D M S}}\right)
$$

where $\mathrm{E}_{\mathrm{PDMS}}$ is $750 \mathrm{kPa}$ [12] and the PDMS, nearly an ideal rubber, is assumed to be incompressable with the Poisson's ration $v_{P D M S}=0.5$. It should be noted that Young's modulus for PDMS is highly dependent on cure time, temperature, and pre-polymer to curing agent ratio, reaching as much as $3 \mathrm{MPa}$ for cure times of 24 hours at $60^{\circ} \mathrm{C}$ [13]. The value chosen here corresponds most closely to the cure conditions used. When released from the substrate, the PDMS contracts, enhancing the compressive stress in the metal layer, and resulting in visible wrinkling of the metal lines as seen in the optical image in Fig. 7.1. Thus, the traces remain in a state of compression even when subjected to considerable tensile strain. 


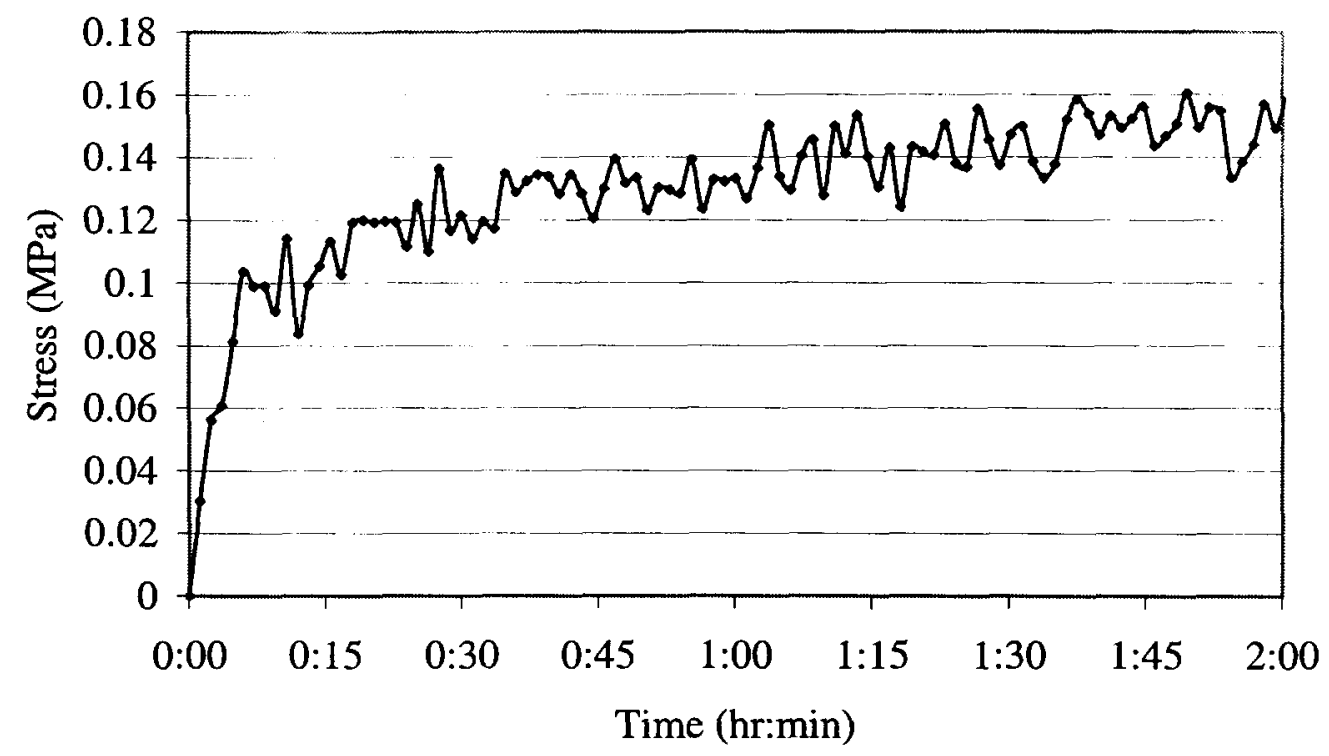

Figure 7.9. Evolution of residual stress during curing of PDMS at $60^{\circ} \mathrm{C}$. The average final residual stress in the PDMS film is $0.15 \mathrm{MPa}$, corresponding to a residual strain of $10 \%$.

\subsubsection{METAL FILM ON PDMS FILM}

From Experiment one it is evident that both adhesion layer thickness and deposition rate affect metal film behavior as seen in Fig. 7.10. The least stressed film is the one with $5 \mathrm{~nm} \mathrm{Ti}$ as the adhesion layer deposited at $0.01 \mathrm{~nm} / \mathrm{sec}$. The film with the largest stress is the $50 \mathrm{~nm} \mathrm{Ti}$ adhesion layer deposited at $0.05 \mathrm{~nm} / \mathrm{sec}$. Experiment two compared the deposition of gold film versus platinum film under the same conditions, and a significant difference is evident in the results as seen in Fig. 7.11. The gold film exhibits wrinkling while the platinum exhibits cracking. 


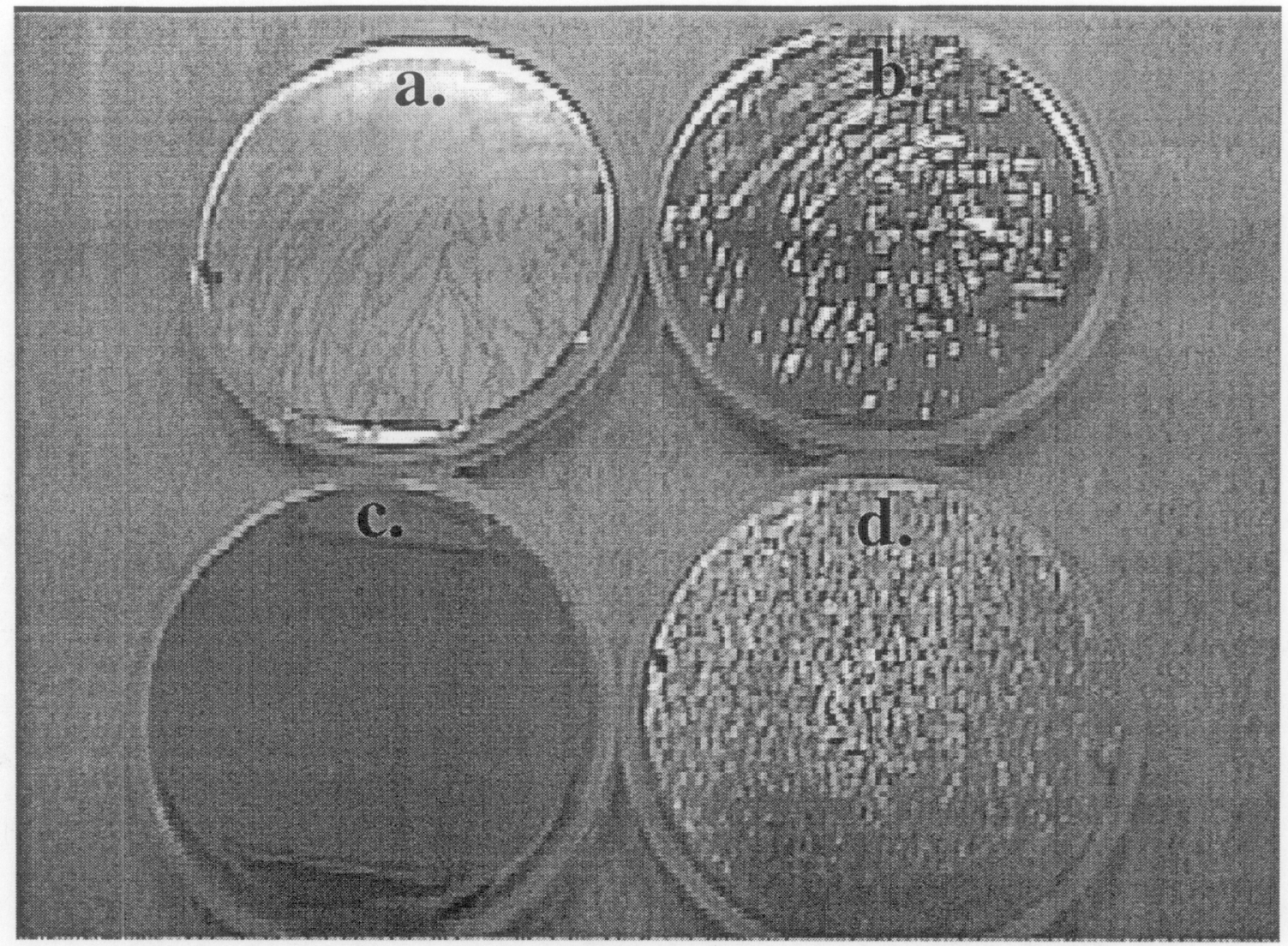

Figure 7.10. Experiment-1 keeping PDMS thickness and gold thickness constant at $50 \mu \mathrm{m}$ and $100 \mathrm{~nm}$ respectively, and varying titanium thickness and deposition rate. (a) 5 $\mathrm{nm} \mathrm{Ti}$ at $0.5 \mathrm{~nm} / \mathrm{sec}$ (b) $50 \mathrm{~nm} \mathrm{Ti}$ at $0.5 \mathrm{~nm} / \mathrm{sec}$ (c) $5 \mathrm{~nm}$ Ti at $0.1 \mathrm{~nm} / \mathrm{sec}$ (d) $50 \mathrm{~nm} \mathrm{Ti}$ at $0.1 \mathrm{~nm} / \mathrm{sec}$
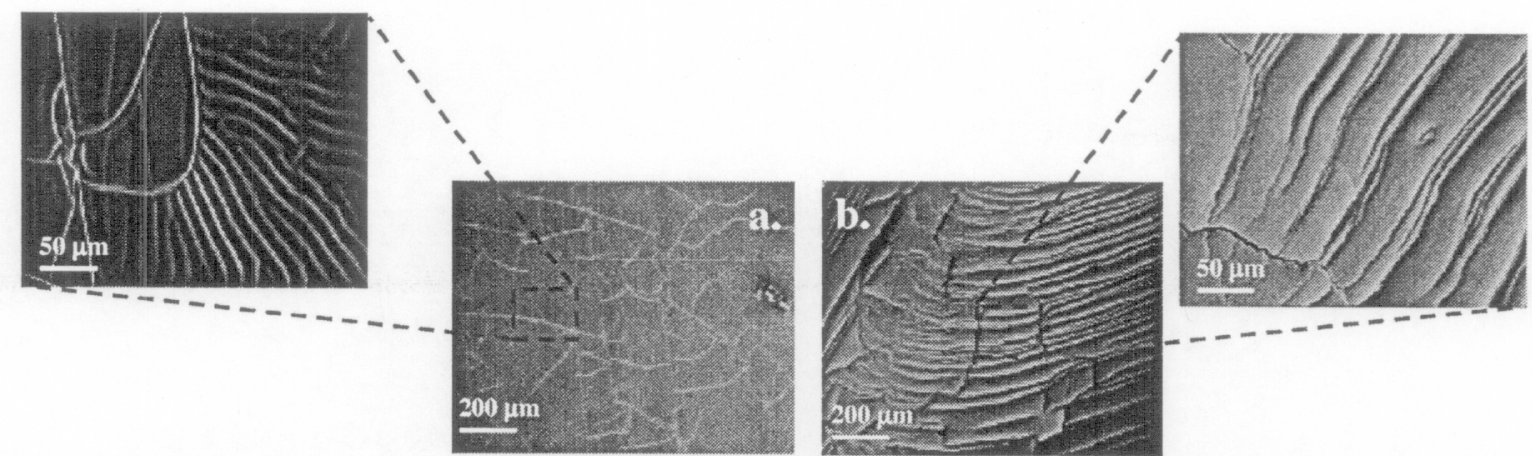

Figure 7.11. Gold versus platinum (a) gold film sputtered on PDMS film with evident wrinkling (b) platinum film sputtered on PDMS film with evident cracking. 


\subsection{DISCUSSION}

Initial manual tensile testing demonstrated that the wrinkled gold films failed at strains averaging $7 \%(\sigma=0.01)[1]$. Subsequent studies performed at Princeton University by Lacour $e t$ al. confirmed the stretchablity of chromium/gold metal films on PDMS substrates. They reported strains up to $22 \%$ for $100 \mathrm{~nm}$ thick gold patterned onto a $1 \mathrm{~mm}$ thick substrate [14]. The discrepancy may be due to the facts that their PDMS layers were considerably thicker than ours, and were not constrained by a rigid substrate. Our automated Instron tensile test average result of 3\% strain to failure was substantially less than our preliminary manual tensile testing results, which raised some concern. When the manual tensile testing was repeated on the new mechanical test samples, it was found that these results, averaging to $6.7 \%$, were consistent with our preliminary manual testing results. The large discrepancy between the manual data and the Instron data is thought to be due to how the sample was gripped during the experimentation procedure. In the Instron, the sample was gripped at the contact pads between two glass pieces with sharp edges, as described in the experimental section. The sharp glass edges may have caused a local stress concentration, causing the metal traces to fail. In the manual stretch test the grips did not overlap the metal traces, and therefore did not compromise the integrity of the metal traces

Although the discrepancy in the absolute strain to failure values between the automated and manual test set-ups was large, the relative failure values between the types of devices were consistent with the two tests. For example, the serpentine leads consistently resulted in higher strain to failure with values of $4.1 \%$ and $8.9 \%$ in the Instron and manual testing, respectively. 
The increase in strain at circuit failure for serpentine versus straight leads can be readily explained if one considers the type of deformation the gold circuit sees during stretching of the electrode array. The curves in the serpentine leads act as a $2 \mathrm{D}$ spring allowing the devices to stretch more than the straight leads. This is modeled using a curved beam analysis in Christina Park's thesis [4]. In a 3D spring the wire is put in torsional bending instead of tension, and the corresponding rotation results in extension of the spring. In our case the serpentine leads behave as a $2 \mathrm{D}$ spring, reducing the axial tensile strain as the traces are now in a combination of tensile and bending deformation. This means much of the deformation on the traces corresponding to stretching the PDMS is taken up by the traces as a rotational motion in the $2 \mathrm{D}$ plane, resulting in increased extension before circuit failure. Assuming that the thin film metal traces fail when a critical tensile stress is applied to the traces, then the maximum strain to failure should occur for $\mathrm{a}^{\circ} 5^{\circ}$ zigzag configuration. For this case, the stress resolved along the trace direction is $1 / 2$ the stress applied axially to the PDMS sample. This corresponds to the minimum stress orientation [15].

During the preliminary stretch tests it was observed that after the leads failed and the stress was relieved, conductivity was regained. This finding was consistently verified with the Instron instrument and is attributed to the viscoelastic nature of PDMS, whereby the imposed strain in the PDMS is eventually recovered once the stress is removed. Regardless of these results, once the devices have been stretched to failure they no longer fit to serve as an implant. The resistance of the metal traces after failure increased significantly. This served as an indicator as to when the devices actually failed even though there was still some ohmic contact between the cracks. 
No direct measurement of extrinsic stresses was performed; since the temperature inside the e-beam could not be monitored or modified. However, in a study conducted by Bowden et al. it is postulated that the compressive stress causing the wrinkling in the metal film deposited on a thick PDMS $(10 \mathrm{~mm})$ is a result purely dependent on the extrinsic effects, or thermal stress $[16,17]$. Extrinsic stress is a factor that cannot be dismissed, since the thermal coefficient of PDMS is so much greater than that of gold. Although in our experiments the e-beam deposition temperature is not known, inspection of Eqn. 7.5 provides the necessary information about the thermal stress present in the film [11].

$$
\sigma_{f}=\tilde{E}_{f}\left(\alpha_{f}-\alpha_{s}\right) \Delta T
$$

The coefficient of thermal expansion (CTE) for the gold film, $\alpha_{\mathrm{f}}$, is only $14.2 \times 10^{-6} / \mathrm{K}$ [18] compared to the much greater value of $960 \times 10^{-6} / \mathrm{K}$ for $\alpha_{s}[14,19]$ of PDMS. To appreciate the thermal effects a sample calculation is performed assuming a temperature difference of $50^{\circ} \mathrm{C}$ and a Young's modulus of $53 \mathrm{GPa}$ for gold films [7]. The extrinsic thermal stress in the gold film was calculated to be $2.5 \mathrm{GPa}$ in compression; corresponding to an extrinsic residual strain of approximately 5\% using Eqn 7.2. This implies that on a free-standing PDMS substrate, the gold film would be in compression and could be stretched $5 \%$ before being put into tension. .

For our devices, we are depositing metal traces $(\sim 100 \mathrm{~nm})$ on thin PDMS films $(\sim 50 \mu \mathrm{m})$ that are spun on thick silicon wafers $(\sim 500 \mu \mathrm{m})$. In this case, the thermal stresses in the metal traces are governed by a combination of both the PDMS and 
substrate coefficient of expansion. Examining the stresses that occur in the PDMS film when cured on a silicon substrate, yields a tensile stress of $25 \mathrm{kPa}$. This corresponds to an extrinsic residual strain of approximately $3 \%$ in the PDMS film on the silicon substrate. This calculation was made using the following assumptions: CTE of silicon and PDMS to be $2.3310^{-6} / \mathrm{K}$ and $960 \times 10^{-6} / \mathrm{K}$ respectively, PDMS Young's modulus of $750 \mathrm{kPa}, 60^{\circ} \mathrm{C}$ for curing temperature and $25^{\circ} \mathrm{C}$ for room temperature.

One must not forget the residual intrinsic stresses that can also contribute to the wrinkling effect in the gold film [6]. A major contributor to the wrinkling is the residual strain in the PDMS film, which is in a state of tensile residual strain resulting from the fact that it is constrained by a rigid substrate while it undergoes a volume contraction associated with the curing process. This residual strain associated with curing was measured to be $10 \%$ as seen in Fig. 7.9 .

From this study we concluded that a combination of the large difference in the thermal coefficient of expansion between metal/PDMS and PDMS/silicon, and the intrinsic stress in the gold film and PDMS film, govern the observed compressive stress; causing the gold leads to wrinkle on the PDMS surface. The conductive traces, which contact the retina, take advantage of residual compressive stress developed during fabrication to eliminate potential damage due to curvature. With these initial results, we believe the devices will be sufficiently robust to withstand handling during fabrication and implantation.

For metals such as $\mathrm{Ti}$ and $\mathrm{Pt}$, we suspect that intrinsic stresses dominate since the deposited films were cracked and not wrinkled, as seen in Figs. 7.10 and $7.11 \mathrm{~b}$. If extrinsic stresses dominated then the Pt and Ti films should also be wrinkled like the 
gold, since both the Pt and Ti also have a much smaller coefficients of expansion (8.9 X $10^{-6} / \mathrm{K}$ and $11.9 \times 10^{-6} / \mathrm{K}[18]$ respectively) in comparison to the PDMS. Often for thin films, intrinsic stresses due to incomplete structural ordering dominate over thermal stresses [6]. The quality of the thin metal films depends on many variables, and stress can be controlled by varying deposition parameters such as temperature, deposition rate, pressure, metal thickness, and adhesion layer thickness to name a few. However, the system that was available for our use only allowed metal thickness and deposition rate control. Other variables that need to be examined in order to understand their effect on mechanical properties are other types of PDMS, different adhesion layers such as chromium, density of leads on the PDMS, and passivation layer. The most significant result from this study was that serpentine leads were found to be significantly more robust than straight leads. Other designs that would be beneficial to try in order to maximize robustness of metal leads are an out of plane serpentine pattern or a corduroy design as seen in Fig. $7.12 \mathrm{a}$ and b.

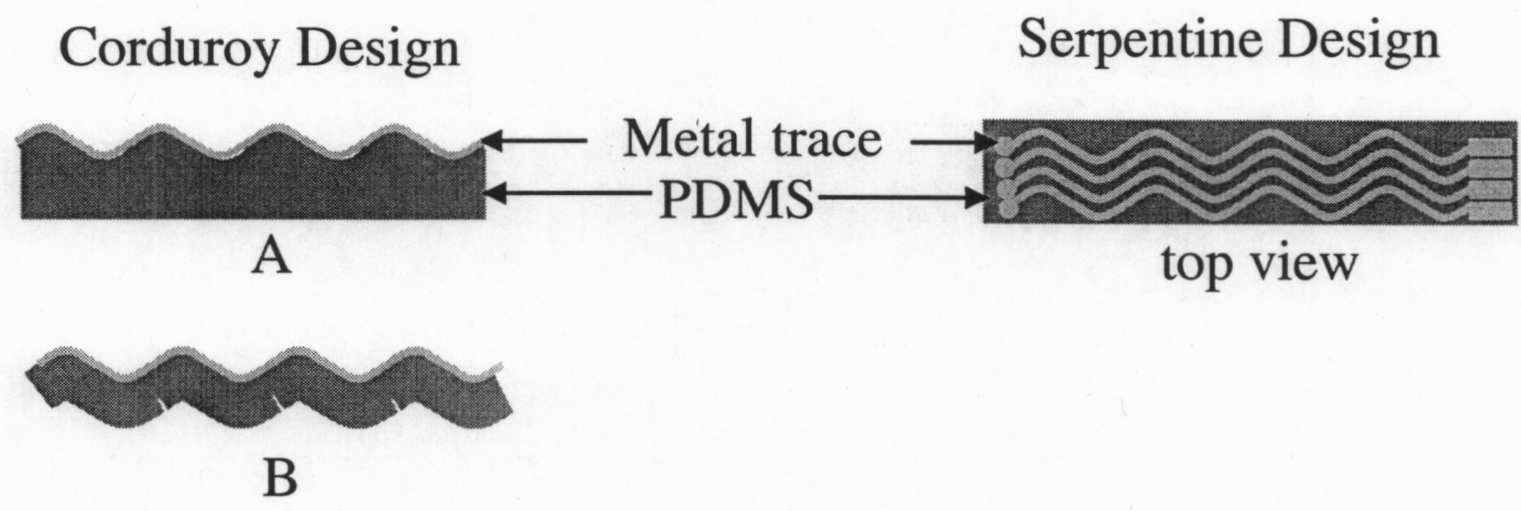

\section{cross-sectional view}

Figure 7.12. Schematic of possible future designs to improve robustness of the next generation devices. 


\subsection{REFERENCES}

[1] M. Maghribi, J. Hamilton, D. Polla, K. Rose, T. Wilson, and P. Krulevitch, "Stretchable Micro-Electrode Array for Retinal Prosthesis," presented at IEEEEMBS special topics n medicine and biology, Madison, WI, 2002.

[2] P. Krulevitch, "Micromechanical investigations of silicon and $\mathrm{Ni}-\mathrm{Ti}-\mathrm{Cu}$ thin films," in Mechanical Engineering. Berkeley: University of California, 1994, pp. 231.

[3] R. W. Hoffman, in Physics of Thin Films, G. Hass and T. E. Thun, Eds., 3 ed. New York: Academic Press, 1966, pp. 211.

[4] C. S. Park, "Characterizing the material properties of polymer-based microelectrode arrays for retinal prosthesis," in Mechanical Engineering: Massachusetts Institute of Technology, 2003, pp. 56.

[5] H. Gray, "Anatomy of the Human Body," vol. 2002: Bartleby.com, 1918.

[6] M. Ohring, Material Science of Thin Films: Deposition \& Structure, Second Edition ed. San Diego: Academic Press, 2002.

[7] H. D. Espinosa and B. C. Prorok, "A Novel Chip Level Test to Identify Elasticity, Plasticity and Fracture of Thin Films and MEMS Materials," vol. 2002: Northwestern University, 2001.

[8] C. H. S. Dupuy and A. Cachard, "Physics of Nonmetallic Thin Films," in NATO Advanced Study Institutes Series: Series B, Physics: Plenum Press, 1976.

[9] G. G. Stoney, Proc. Roy. Soc. London Ser. A, vol. 82, pp. 172, 1909.

[10] S. Timoshenko, "Analysis of Bi-metal Thermostats," J. Opt. Soc. Am., vol. 11, pp. $233,1925$.

[11] M. Madou, Fundamentals of Microfabrication. New York: CRC, 1997. 
[12] D. Armani, C. Liu, and N. Aluru, "Re-configurable fluid circuits by PDMS elastomer micromachining," 1999.

[13] B. Michel, A. Bernard, A. Bietsch, E. Delamarche, M. Geissler, D. Juncker, H. Kind, J. P. Renault, H. Rothuizen, H. Schmid, P. SchmidtWinkel, R. Stutz, and H. Wolf, "Printing meets lithography: Soft approaches to high-resolution patterning (vol 45, pg 697, 2001)," Ibm Journal of Research and Development, vol. 45, pp. $870,2001$.

[14] S. P. Lacour, S. Wagner, Z. Haung, and Z. Suo, "Stretchable gold conductors on elastomeric substrates," Applied Physics Letters, vol. 82, pp. 2404-2406, 2003.

[15] E. P. Popov, Engineering mechanics of solids, 2nd ed. Upper Saddle River: Prentice Hal, 1998.

[16] N. Bowden, S. Brittain, A. G. Evans, J. W. Hutchinson, and G. Whitesides, "Spontaneous formation of ordered structures in thin films of metals supported on a elastomeric polymer," Nature, vol. 393, pp. 146-149, 1998.

[17] W. T. S. Huck, N. Bowden, P. Onck, T. Pardoen, J. W. Hutchinson, and G. M. Whitesides, "Ordering of spontaneously formed buckles on planar surfaces," Langmuir, vol. 16, pp. 3497-501, 2000.

[18] Metals Handbook. Materials Park: ASM International, 1998.

[19] Dow Corning Corporation, "Sylgard 184," Midland MI 2002. 


\section{CHAPTER 8: CONCLUSION \& FUTURE DIRECTIONS}

According to the requirements set by our collaborators and application specifications, PDMS devices were designed, fabricated, and tested. The use of a PDMS substrate required that new fabrication techniques be developed. Multiple process iterations were required to establish the most appropriate process flow. Although these iterations were time intensive they led to innovative techniques that will be critical in the development of the final implant array. First and second generation device testing showed that PDMS appears to meet the critical requirements for microelectrode array. The conformability and flexibility of the PDMS devices have been crucial characteristics in terms facilitating surgical insertion and making uniform contact with the retina. At the same time the devices were found to be robust enough to withstand relatively large deflections and surgical manipulation without inducing failure. The ability to batchfabricate the devices has been advantageous in terms of achieving high yield and reproducibility at a reasonable cost. Key challenges that were overcome during the fabrication development include: 1) isolating and eliminating surface contamination issues, 2) generating reproducible processing techniques with high yield, and 3) providing a reliable interconnect to interface with external electronics.

The pioneering progress reported herein on the silicone-based technology development for producing the microelectrode array for an epiretinal prosthesis prototype system provides exciting entrance to extended research and applications. The advancements made in silicone-based microfabrication provides a technology platform to enable the development of a high-density micrelectrode array. The $1^{\text {st }}$ and $2^{\text {nd }}$ generation microelectrode arrays were developed to establish basic engineering feasibility. The final 
implant specifications calls for a device with approximately a1000 electrodes contained within an area of $16 \mathrm{~mm}^{2}$. An OctoPDMS device is proposed to achieve this goal. The electrode array would be contained in the octagonal base with eight arms containing 125 metal traces for electrical contact. The base will lie against the retina while the arms conform to the curvature of the eye and converge onto an IC chip in the region where the lens usually resides as seen in Fig. 8.1.

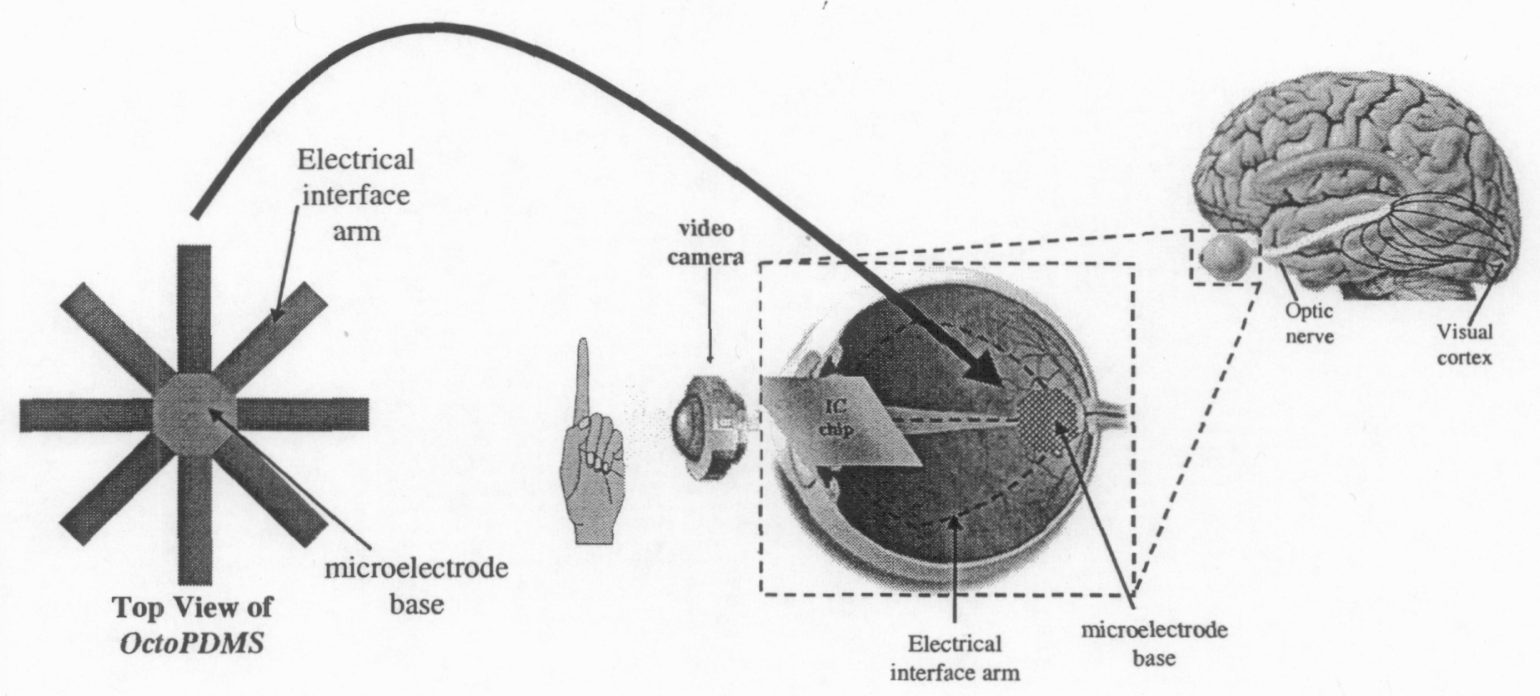

Figure 8.1. OctoPDMS overall concept. Figure adapted from [1-3]

Long-term biocompatibility studies are currently being performed to ensure life long durable implants. The main obstacle to the implantation will be the maintenance of the implant inside the body. It is prerequisite that the implant remains viable and biocompatible for the entire life of the recipient.

Several prospects exist for future work on the implantable microelectrode array. One example is investigating affects of different plasma treatments on bonding and metalization, as such treatment appears to promote adhesion by producing reactive chemical groups on the surface. We hypothesize that best bonding will result from 
tailoring the reactive groups on the surface to meet specific adhesion needs. This may be accomplished by varying the type of plasma used in order to produce the most thermodynamically favorable reaction. Another opportunity for future work lies in optimization of the PDMS metalization process. This can be accomplished by parametric deposition studies. Parameters include deposition rate, temperature, pressure, thickness and special design variables such as shape, density, and size. Further investigation is warranted into varying size, shape, and density of the reinforcement ribs to design arbitrary curvature into the PDMS devices. This can potentially allow the radius of curvature of the devices be tailored to match that of the eye hence minimizing stress exerted on the retina and maximizing uniform contact.

The medical purpose of the retinal implant is strictly to give enough sight to the blind in order to make their lives more self-sufficient. Researchers are exploring and have yet to discover methods to provide depth perception, color, and contrast to the images created by the implant. A grand challenge still exists to develop an implant capable of providing complete natural vision. However the great success of the cochlear implant is proof that a neural implant can make a significant impact despite its simplicity in comparison to natural biological system. There is no doubt that an implantable retina will have a significant impact on our society as it helps to alleviate some forms of blindness. While experiments to date using this new implant technology have all been promising, there is still substantial research effort required to produce a fully functional implant. Providing sight to the visually impaired through retinal prosthesis will be a phenomenal application of silicone-based microtechnology. 


\section{REFERENCES}

[1] M. Erickson, "http://www.stlukeseye.com." 2003.

[2] B. Veisland, "http://wwwVeisland.com." 2003.

[3] DOE, "A High-Density Microelectronic-Tissue Hybrid Sensor for Imaging," DOE LAB 01-14 Biomedical Engineering Program, 2001. 